\title{
Applications of SHARP Toolkit to SFR Challenging Problems: Evaluation of Hot Channel Factors and Demonstration of Zooming Capability
}

Nuclear Science and Engineering Division 


\title{
About Argonne National Laboratory
}

Argonne is a U.S. Department of Energy laboratory managed by UChicago Argonne, LLC under contract DE-AC02-06CH11357. The Laboratory's main facility is outside Chicago, at 9700 South Cass Avenue, Argonne, Illinois 60439. For information about Argonne and its pioneering science and technology programs, see www.anl.gov.

\section{DOCUMENT AVAILABILITY}

Online Access: U.S. Department of Energy (DOE) reports produced after 1991 and a growing number of pre-1991 documents are available free at OSTI.GOV (http://www.osti.gov/), a service of the US Dept. of Energy's Office of Scientific and Technical Information.

\author{
Reports not in digital format may be purchased by the public from the \\ National Technical Information Service (NTIS): \\ U.S. Department of Commerce \\ National Technical Information Service \\ 5301 Shawnee Rd \\ Alexandria, VA 22312 \\ www.ntis.gov \\ Phone: (800) 553-NTIS (6847) or (703) 605-6000 \\ Fax: (703) 605-6900 \\ Email: orders@ntis.gov
}

Reports not in digital format are available to DOE and DOE contractors from the Office of Scientific and Technical Information (OSTI):

U.S. Department of Energy

Office of Scientific and Technical Information

P.O. Box 62

Oak Ridge, TN 37831-0062

www.osti.gov

Phone: (865) 576-8401

Fax: (865) 576-5728

Email: reports@osti.gov

\section{Disclaimer}

This report was prepared as an account of work sponsored by an agency of the United States Government. Neither the United States Government nor any agency thereof, nor UChicago Argonne, LLC, nor any of their employees or officers, makes any warranty, express or implied, or assumes any legal liability or responsibility for the accuracy, completeness, or usefulness of any information, apparatus, product, or process disclosed, or represents that its use would not infringe privately owned rights. Reference herein to any specific commercial product, process, or service by trade name, trademark, manufacturer, or otherwise, does not necessarily constitute or imply its endorsement, recommendation, or favoring by the United States Government or any agency thereof. The views and opinions of document authors expressed herein do not necessarily state or reflect those of the United States Government or any agency thereof, Argonne National Laboratory, or UChicago Argonne, LLC. 


\section{Applications of SHARP Toolkit to SFR Challenging Problems: Evaluation of Hot Channel Factors and Demonstration of Zooming Capability}

prepared by

E. R. Shemon, Y. Yu, and T. K. Kim

Nuclear Engineering Division, Argonne National Laboratory

September 30, 2018 



\section{EXECUTIVE ABSTRACT}

The Simulation-based High-efficiency Advanced Reactor Prototyping (SHARP) toolkit is under development by the Nuclear Energy Advanced Modeling and Simulation (NEAMS) Campaign of the U.S. Department of Energy, Office of Nuclear Energy. Beginning in FY17, the NEAMS Campaign initiated the "Sodium-Cooled Fast Reactor (SFR) Challenge Problems" task to apply its advanced modeling and simulation capabilities to important SFR applications. The scope of work includes two major items: the assessment of hot channel factors (HCFs) and the demonstration of a local refinement "zooming" capability, both using the SHARP toolkit. Resolving these challenge problems with the SHARP toolkit can significantly improve the economic competitiveness of an SFR as well as make the tools more accessible for full core confirmatory calculations.

This project developed computational models, meshes, and coupling procedures for highfidelity multi-physics calculations using the neutronics (PROTEUS) and thermal-hydraulic (Nek5000) components of the SHARP toolkit in order to evaluate HCFs for the 100 MWe Advanced Fast Reactor (AFR-100). The feasibility of the SHARP zooming capability was demonstrated for the AFR-100 design by focusing on a pin-by-pin model of a fuel assembly at focal regions in a full core calculation.

The HCFs developed for the earlier SFRs (FFTF, CRBR, and EBR-II) were reviewed, and a subset of these were identified as potential candidates for reduction or elimination through high-fidelity simulations. A one-way offline coupling method was used to evaluate the HCFs where the neutronics solver PROTEUS computes the power profile based on an assumed temperature, and the computational fluid dynamics solver Nek5000 evaluates the peak temperatures using the neutronics power profile. This one-way offline method is valid because the neutronics power profile has weak dependence on small temperature variation. In this study, different temperature profiles were used in neutronics for comparison including flat temperature profiles and a more accurate temperature profile from a STAR-CCM+ calculation. The temperature profile used was found to have little to no effect on the pin-wise axial power distribution communicated to Nek5000 for the HCF calculation. If more detailed neutronics information or eigenvalue is desired, online iterations between the PROTEUS and Nek5000 is required, but it is impractical at this moment due to the limited computation resources.

The HCFs of the peak temperatures at cladding outer surface, cladding inner wall surface, and fuel centerline induced by cladding manufacturing tolerance, fissile content maldistribution, and uncertainties on the cladding, coolant, and fuel properties were evaluated for the AFR-100. An assessment of the effect of wire wrap configuration and size of the bundle shows that it is practical to use a small 7-pin bare rod bundle to calculate the HCFs for most cases. The resulting HCFs obtained from the high-fidelity SHARP calculations are generally smaller than those developed for the earlier SFRs because uncertainties involved in the 
modeling and simulations were reduced, removing some conservatism due to modeling uncertainty.

The SHARP zooming concept reduces computational expense of a full core simulation while maintaining pin-level accuracy in focal regions of the core. The validity of the zooming concept was proven by comparing calculations with successively increased geometrical heterogeneity around a single focal assembly. The PROTEUS neutronics calculations were performed for full core models with homogeneous assemblies except for heterogeneous pin-by-pin assemblies at the focal regions. Since the full core neutronics simulation provided accurate neutron current distributions at the surfaces of the focal assembly, the PROTEUS neutronics calculations could provide accurate power distributions in the focal assembly regardless of the geometrical approximations in surrounding assemblies. This allows the mesh complexity and subsequently the cost of the full core simulation to be substantially reduced. Comparison of several Nek5000 calculations demonstrated that the thermal-hydraulic properties in ducted SFR fuel assemblies are sufficiently isolated from neighboring assemblies, and as a result, the heterogeneous single assembly calculations with the Nek5000 were sufficient to obtain pin level temperature distributions in the focal regions. 


\section{TABLE OF CONTENTS}

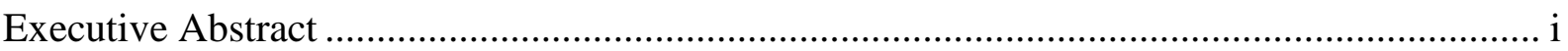

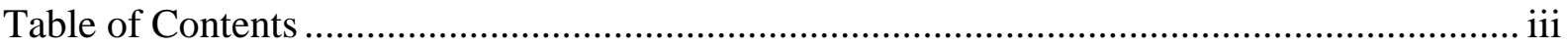

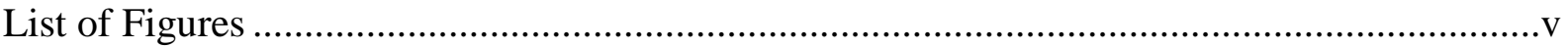

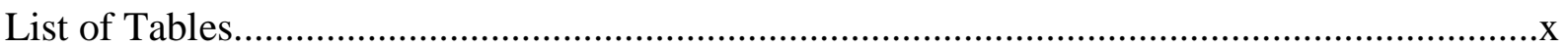

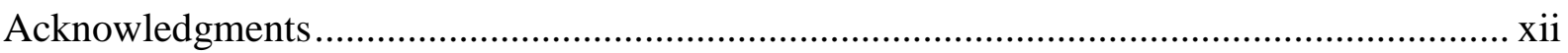

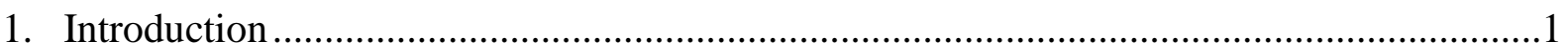

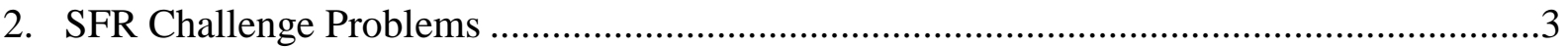

2.1 Assessment of Hot Channel Factors …………………..............................................

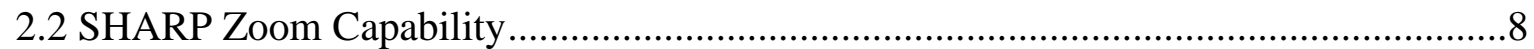

3. SFR Design Problems and Computational Tools............................................................11

3.1 Advanced Fast Reactor as SFR Design Problem ........................................................11

3.2 Computational Tools and Resources.......................................................................13

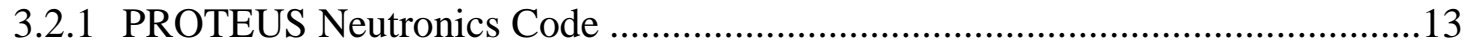

3.2.2 Nek5000 Thermal Hydraulics Code ............................................................14

3.2.3 Multiphysics Coupling Approach and Computational Resources.......................16

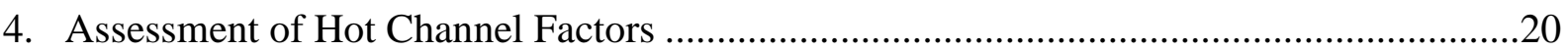

4.1 Hot Channel Factors for Metallic Fuel SFRs ................................................................20

4.2 Strategy for Hot Channel Factor Assessments ...........................................................21

4.3 Neutronics Results for Hot Channel Factor Calculations .............................................24

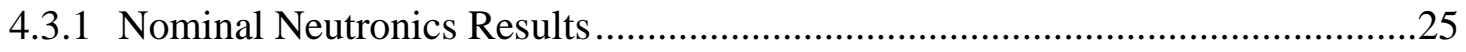

4.3.2 Neutronics Results Associated with Uncertainties in Thermo-Physical Properties .....................................................................................

4.3.3 Neutronics Results Associated with Uncertainties in Subchannel Flow Area ...30

4.3.4 Neutronics Results Associated with Uncertainties in Fuel Pellet Fissile

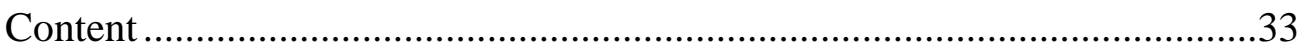

4.4 Thermal-Hydraulic Results for Hot Channel Factor Calculations ...................................38

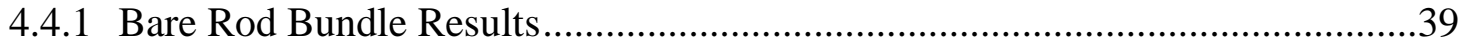

4.4.2 Wire Wrap Rod Bundle Results .......................................................................48

4.5 Estimation of Hot Channel Factors ...........................................................................

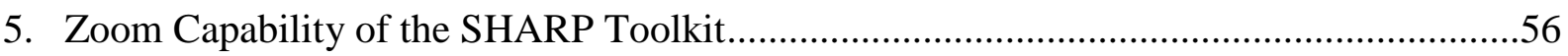


5.1 Application of Zoom Capability to AFR-100 Peak Temperature Calculations .............56

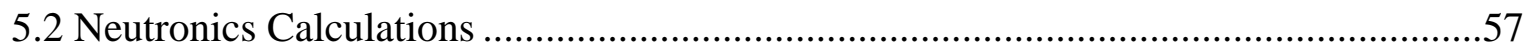

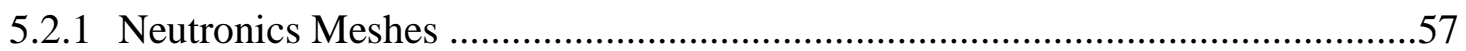

5.2.2 Multigroup Cross Section Generation Procedure ..............................................59

5.2.3 Code-to-Code Verification with Legacy Solver ..................................................60

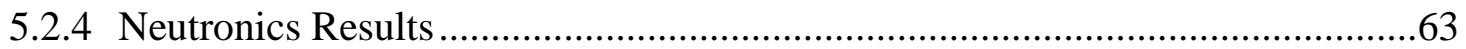

5.2.5 Neutronics Zooming Summary ………………….......................................67

5.3 Thermal Hydraulics Calculations..........................................................................68

5.3.1 Thermal Hydraulic Model ...........................................................................68

5.3.2 Thermal Hydraulic Results .......................................................................

5.3.3 Thermal Hydraulic Zooming Summary ………...........................................75

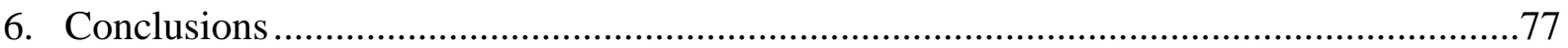

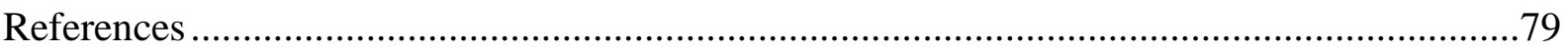

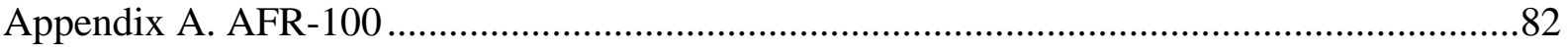

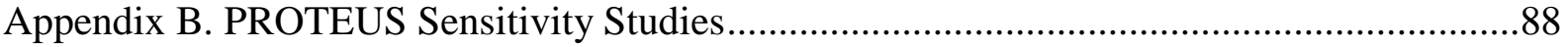

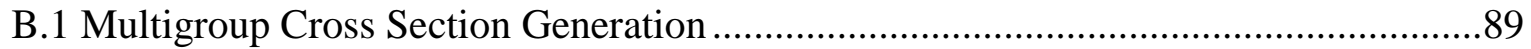

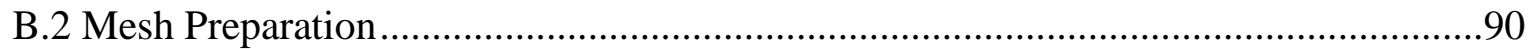

B.3 Transport Solvers: PROTEUS-SN vs. PROTEUS-MOCEX ………………................91

B.4 Mesh, Angle and Energy Convergence Studies ...........................................................92

B.4.1 Axial Mesh Refinement................................................................................93

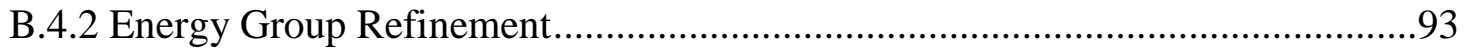

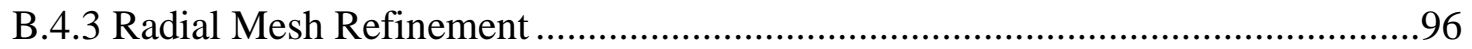

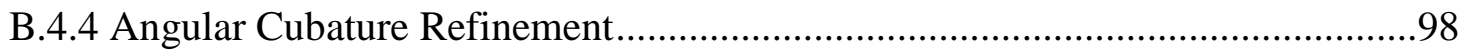

B.4.5 Convergence Study Conclusions .........................................................................99

B.5 Discussion of PROTEUS-SN vs. PROTEUS-MOCEX Results .....................................99

B.6 Discussion of Initial Temperature Condition ..........................................................101

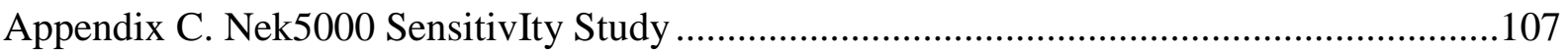

C.1. Primary STAR-CCM+ calculations ………………………………………….....107

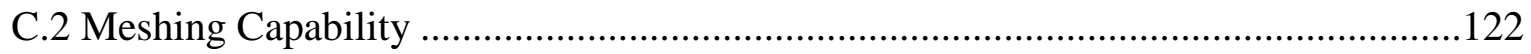

Appendix D. Comparison of Hot Channel Factors .................................................................125 


\section{LIST OF FIGURES}

Figure 2.1.1 Schematic of assessment of margin to fuel-cladding eutectic formation .............. 3

Figure 2.1.2 SFRs operated or designed in the United States ............................................ 4

Figure 2.1.3 Evolution of computational resources and codes ....................................... 6

Figure 2.1.4 Peak cladding inner wall temperature depending on HCFs.............................. 8

Figure 2.2.1 Americium-bearing blanket (AmBB) concept................................................. 9

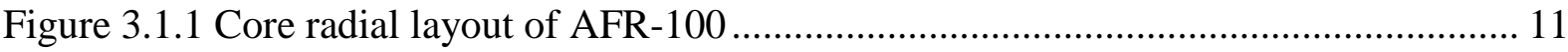

Figure 3.1.2 Core axial layout and uranium enrichment distribution ................................. 12

Figure 3.2.2.1 Comparison of convergence behavior between Nek5000 and OpenFOAM

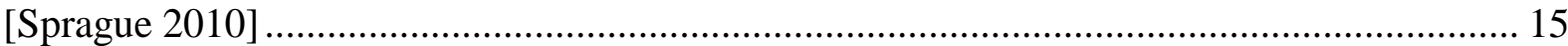

Figure 3.2.2.2 Hierarchical structure of NEAMS TH tools ........................................... 16

Figure 3.2.3.1 Schematic of online coupling using the SHARP toolkit .............................. 17

Figure 3.2.3.2 Schematic of offline coupling using components of the SHARP toolkit........ 17

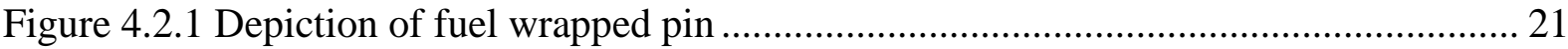

Figure 4.2.2 Close-up mesh of 91-Pin fuel assembly and varying sub-channel geometry ...... 22 Figure 4.2.3 Nek5000 simulation for of 217-pin assembly with over 1 million grid points

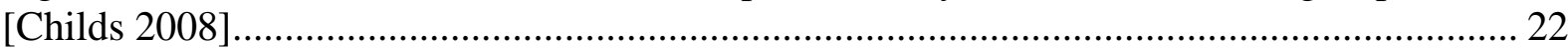

Figure 4.2.4 7-Pin (left) and 91-pin (right) configurations ............................................ 23

Figure 4.3.1.1. MOCEX nominal condition axial power profile (7-pin) ............................ 26

Figure 4.3.1.2. MOCEX nominal condition axial power profile (91-pin) ............................ 26

Figure 4.3.1.3 Axial power profile comparison between DIF3D and MOCEX (7-pin) ......... 28

Figure 4.3.1.4 Axial profiles for computed by 33g MOCEX simulation (7-pin) .................. 29

Figure 4.3.1.5 Fission cross section for U-235 retrieved (from National Nuclear Data Center website)

Figure 4.3.3.1 Overlay of the initial and perturbed meshes to show cladding perturbation ($0.05 \mathrm{~mm}$ change in cladding thickness).

Figure 4.3.3.2 MOCEX axial power profile for cladding thickness change (7-pin).............. 32

Figure 4.3.3.3 MOCEX axial power profile for cladding thickness change (91-pin)............ 32

Figure 4.3.4.1. Eigenvalue distribution based on 30 random samples................................ 34

Figure 4.3.4.2. Peak pin power distribution based on 30 random samples........................... 34

Figure 4.3.4.3. Hypothetical peak pin power distribution based on random sampling with a very large number of samples. 
Figure 4.3.4.4. Normal distribution of enrichment variation for Simulation 25................... 36

Figure 4.3.4.5. Normalized axial power distribution for all 91 pins within Simulation 25.... 37

Figure 4.3.4.6. Power distribution at active core outlet from nominal condition (left) and randomized fissile content bounding case (right)................................................... 38

Figure 4.4.1.1 Three axial power distributions applied to 7-pin bare rod bundle.................. 40

Figure 4.4.1.2 Temperature distributions at different Elevations (4.0P, 4.2P, 4.4P, 4.6P, 4.8P, 5.0P) in 7-pin bare rod bundle using PROTEUS power distribution.................................... 41

Figure 4.4.1.3 Outlet temperature distributions with different axial power distribution in 7-pin

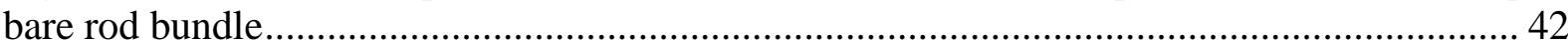

Figure 4.4.1.4 Numbering scheme for sub-channels and rods in 7-pin bare rod bundle ......... 43

Figure 4.4.1.5 Maximum temperature for different sub-channels and rods in 7-pin bare rod

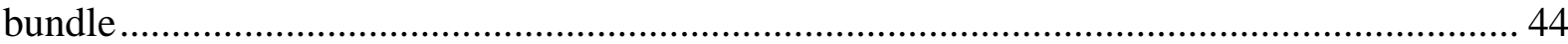

Figure 4.4.1.6 Outlet velocity and temperature distribution in 91-pin bare rod bundle ......... 45

Figure 4.4.1.7 Rod numbering scheme in 91-pin bare rod bundle...................................... 46

Figure 4.4.1.8 Maximum temperature for different rods in 91-pin bare rod bundle............... 47

Figure 4.4.2.1 Comparison of outlet velocity distribution between 91-pin bare rod bundle and

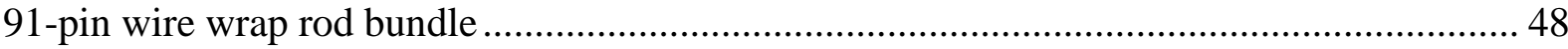

Figure 4.4.2.2 Sub-channel maximum coolant temperature between 7-pin bare rod bundle and

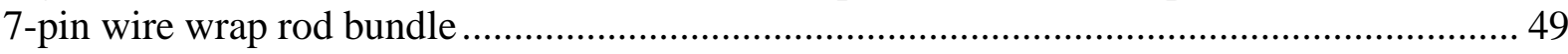

Figure 4.4.2.3 Maximum outer cladding temperature between 91-pin bare rod bundle and 91-

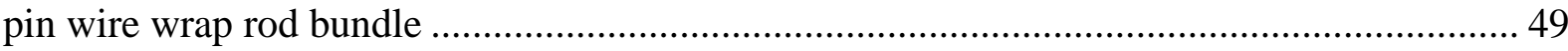

Figure 4.4.2.4 Workflow of tet-to-hex meshing strategy ............................................... 51

Figure 4.4.2.5 Velocity distribution with different wire orientation from Nek5000 and

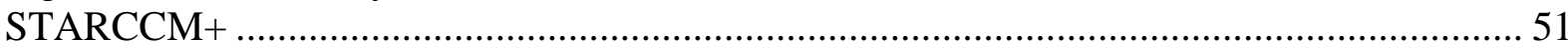

Figure 4.4.2.6 Comparison of outlet velocity distribution with same and different wire

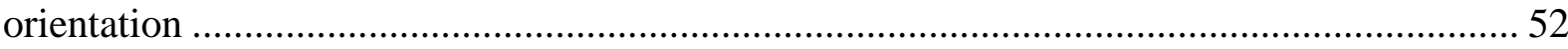

Figure 4.4.2.7 Comparison of outlet temperature distribution with same and different wire

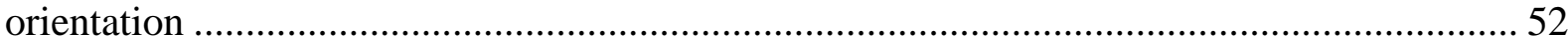

Figure 5.1.1 AFR-100 modeling for SHARP zoom calculation ...................................... 57

Figure 5.2.1.1 Homogeneous AFR-100 mesh showing focal assembly in center of right image. 58

Figure 5.2.1.2 Single-heterogeneous assembly AFR-100 mesh showing focal assembly in

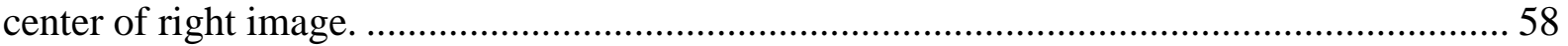

Figure 5.2.1.3 Seven-heterogeneous assembly AFR-100 mesh showing focal assembly in center of right image. 
Figure 5.2.1.4 Seven-heterogeneous assembly AFR-100 mesh showing details of focal assembly and surrounding assembly.

Figure 5.2.3.1 DIF3D vs. PROTEUS: Power distribution (W/cc) in axial slices of active core: (top) outlet at $\mathrm{z}=216 \mathrm{~cm}$, (middle) center at $\mathrm{z}=156 \mathrm{~cm}$, (bottom) and inlet at $\mathrm{z}=97 \mathrm{~cm}$. 62

Figure 5.2.4.1 Radially-integrated axial power distribution (W/cm) in focal assembly for various mesh models.

Figure 5.2.4.2 Power distribution (W/cc) in axial slices of active core: (top) outlet at $\mathrm{z}=216$ $\mathrm{cm}$, (middle) center at $\mathrm{z}=156 \mathrm{~cm}$, (bottom) and inlet at $\mathrm{z}=97 \mathrm{~cm}$.

Figure 5.2.4.3 (left) Pin power (W) in each pin of focal assembly; (right) Pin numbering scheme. 66

Figure 5.2.4.4 (Left) Linear power (W/cm) in selected pins of focal assembly; (right) Pin numbering scheme. 66

Figure 5.2.4.5 Normalized linear power in all pins of focal assembly. 67

Figure 5.3.1.1 The mesh of 7-assembly model 69

Figure 5.3.1.2 Maximum fuel temperature of 7-assembly model and single assembly model 69 Figure 5.3.1.3 Total power for each assembly from three models.......................................... 70

Figure 5.3.1.4 The numbering scheme and velocity distribution for 7-assembly model......... 70

Figure 5.3.2.1 Temperature distribution at active core outlet of focal assembly with homogeneous model heterogeneous neutronics models 71

Figure 5.3.2.2 Temperature distribution at active core outlet of 7 assemblies using heterogeneous neutronics model .......................................................................................... 71

Figure 5.3.2.3 Cladding and fuel temperature distributions in focal assembly........................ 73

Figure 5.3.2.4 Hot zone prediction with homogeneous mode and hot spot prediction with heterogeneous model.

Figure 5.3.2.5 Absolute cladding and fuel temperature deviation in focal assembly between homogeneous model and heterogeneous model (top) and pin numbering (bottom)................ 75

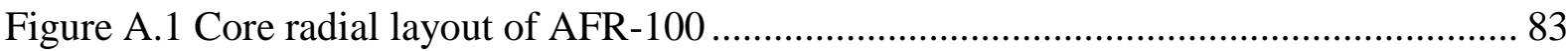

Figure A.2 Core axial layout and uranium enrichment distribution ........................................ 84

Figure A.3 Radial power distribution (MW per assembly in 1/3 Core)................................... 86

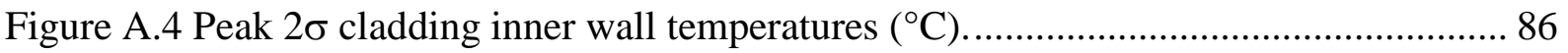

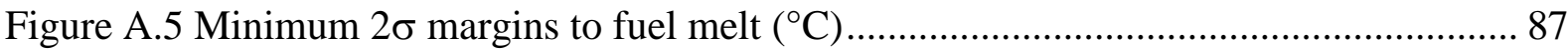

Figure B.1.1 Cross section generation procedure for heterogeneous 3D pin configurations .. 90 Figure B.1.2 General depiction of assembly into 1-D cylinder geometry (not to scale) ......... 90 Figure B.2.1 Visualization of various neutronics meshes for the 7-pin configuration ............. 91 
Figure B.4.2.1 MOCEX axial power profile with varying group structure (7-pin) ............... 94

Figure B.4.2.2 MOCEX axial power profile for uniform enrichment problem (7-pin).......... 95

Figure B.4.2.3 MOCEX axial power profile with varying group structure (91-pin) ............. 96

Figure B.4.2.4 MOCEX ring-wise axial power profiles with 33-groups (91-pin)................ 96

Figure B.4.3.1 Converged 2D neutronics mesh (265 elements) for the 7-pin configuration... 97

Figure B.4.3.2 Converged 2D neutronics mesh (3061 elements) for the 91-pin configuration98

Figure B.5.1 MOCEX vs. SN axial power profile comparison for uniform $300 \mathrm{~K}$ (91-pin). 101

Figure B.6.2 MOCEX comparison of power profile at 300K and $800 \mathrm{~K}$ (91-pin). 103

Figure B.6.3 MOCEX and SN axial power distributions for different temperature initial conditions (91-pin) 105

Figure B.6.4 MOCEX and SN axial power distributions for different temperature initial conditions, zoomed in at upper 18.0\% enriched core region to show detail (91-pin).......... 106

Figure C.1.1 Snapshot of polyhedral mesh for wire wrap rod bundle .............................. 107

Figure C.1.2 Verifications with STAR-CCM+ ........................................................... 109

Figure C.1.3 Boundary conditions for full length domain and 1wire wrap pitch domain ..... 110

Figure C.1.4 Mesh structure for wire wrap rod bundle with cladding and fuel .................. 111

Figure C.1.5 Outlet temperature distributions of full length 91-pin wire wrap rod bundle with conjugate heat transfer

Figure C.1.6. Outlet velocity contour for full length and 1 pitch. 112

Figure C.1.7 Detailed velocity distribution on the line between rods for full length and 1 pitch

Figure C.1.8 Minimum, average and maximum coolant temperature comparison on each elevation between full length and 1 pitch

Figure C.1.9 Normalized axial power distribution for BOC, MOC and EOC of AFR-100 .. 114

Figure C.1.10 Maximum coolant temperature comparison at different elevations for full length and 1 Pitch models with BOC axial power distribution........................................ 115

Figure C.1.11 Lateral velocity distribution ............................................................ 116

Figure C.1.12 Asymmetric distribution feature of hot spots in wire wrap rod bundle ......... 117

Figure C.1.13 Maximum coolant temperature with difference models in different subchannels

Figure C.1.14 Sketch of fuel and cladding ............................................................. 119

Figure C.1.15 Temperature prediction with and without conjugate heat transfer ............... 121

Figure C.1.16 Circumferential conduction in cladding and fuel. 
Figure C.1.17 Circumferential cladding temperature distribution of the center pin with different cladding thickness

Figure C.2.1 Hexahedral mesh generation for wire wrap rod bundle 123

Figure C.2.2 Mesh Generation for bare rod bundle with ICEM script 124

Figure C.2.3 Mesh generation for different physics codes 124 


\section{LIST OF TABLES}

Table 2.1.1 Comparison of HCFs related to cladding mid-wall temperature ............................ 5

Table 3.1.1 Core performance parameters .......................................................................... 12

Table 3.2.1.1 Strong spatial scalability of PROTEUS-SN on BlueGene/P (ANL) ................. 14

Table 3.2.1.2. Weak angle scalability of PROTEUS-SN on BlueGene/P (ANL, JSC) ............ 14

Table 4.1.1 Proposed hot channel factors for metallic fuel SFR ........................................... 20

Table 4.2.1. Required high-fidelity calculations for HCF evaluations ................................... 24

Table 4.3.1.1. MOCEX nominal condition average pin power in each ring (91-pin) ............. 27

Table 4.3.3.1 Cold cladding thickness perturbations and corresponding hot dimensions ....... 30

Table 4.3.3.2 MOCEX eigenvalue results for cladding perturbation (7-pin) .......................... 31

Table 4.3.3.3 MOCEX eigenvalue results for cladding perturbation (91-pin) ......................... 31

Table 4.5.1 Hot channel factors in bare rod bundle and wire wrap rod bundle ....................... 53

Table 4.5.2 Evaluation of hot channel factors for selected uncertainties.................................. 54

Table 4.5.3 Evaluation of temperature rises (degrees K) in hot channels for selected

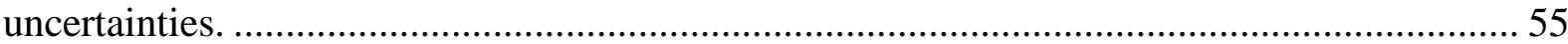

Table 4.5.4 Equations to compute coolant, cladding, and fuel hot channel factors ................. 55

Table 5.2.1.1 Neutronics meshes used in zooming calculations............................................ 57

Table 5.2.3.1 K-effective comparison for homogeneous assembly calculation. ....................... 61

Table 5.2.3.2 Integrated power comparison for homogeneous assembly calculation. ............. 61

Table 5.2.4.1 K-effective comparison for all neutronics zooming calculations. ...................... 63

Table A.1 Core performance parameters............................................................................. 84

Table B.1 Hot radial dimensions for AFR-100 fuel assembly.............................................. 88

Table B.2 Hot axial dimensions for AFR-100 fuel assembly ……………………................. 88

Table B.3.1 Comparison of PROTEUS-SN and PROTEUS-MOCEX neutronics solvers...... 92

Table B.4.1.1 MOCEX eigenvalues with varying degrees of axial refinement (7-pin)........... 93

Table B.4.2.1 MOCEX eigenvalues with varying group structures (7-pin) ............................ 93

Table B.4.2.2 MOCEX eigenvalue results with varying group structures (91-pin) ................ 95

Table B.4.3.1 MOCEX eigenvalue results with varying azimuthal subdivisions (7-pin) ....... 97

Table B.4.3.2 MOCEX eigenvalue results with varying radial subdivisions (7-pin) .............. 97

Table B.4.3.3 MOCEX results with varying radial and azimuthal subdivisions (91-pin) ....... 97

Table B.4.4.1 MOCEX eigenvalue results with varying angular cubature (7-pin).................. 98

Table B.4.4.2 MOCEX eigenvalue results with varying angular cubature (91-pin)................ 99 
Table B.4.5.1 Recommended MOCEX discretization parameters

Table B.5.1 MOCEX vs. SN eigenvalue results for uniform $300 \mathrm{~K}$ temperature (91-pin) ... 100

Table B.6.1 MOCEX eigenvalue results at two uniform temperatures (7-pin) ..................... 102

Table B.6.2 MOCEX eigenvalue results at two uniform temperatures (91-pin) ................... 102

Table B.6.3 MOCEX peak/average pin power in each ring at two uniform temperatures (91-

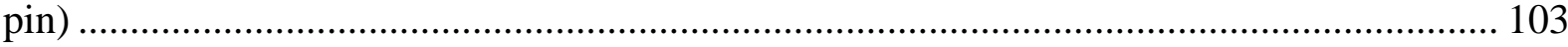

Table B.6.3 Estimated temperature ranges for 7-pin and 91-pin configurations ................... 103

Table B.6.4 MOCEX and SN peak / average axial power results for temperature profile initial condition (91 pin) ......................................................................................................... 104

Table C.1.1 Maximum coolant temperature with difference models.................................... 118 


\section{ACKNOWLEDGMENTS}

This research used resources of the Argonne Leadership Computing Facility, which is a DOE Office of Science User Facility supported under Contract DE-AC02-06CH11357. The authors thank the DOE ASCR Leadership Computing Challenge (ALCC) program for the 2017-2018 computing award of 44 million core-hours awarded on ALCF's BG/Q machine.

The authors gratefully acknowledge the computing resources provided on Blues, a highperformance computing cluster operated by the Laboratory Computing Resource Center at Argonne National Laboratory.

The authors thank Dr. T. Sofu, J. Thomas, and A. Tentner of Nuclear Engineering Division of ANL for their valuable comments on the SFR challenge problems and the applications of the SHARP toolkit. The authors thank A. Mausolff of the University of Florida (supported under a Nuclear Energy University Program fellowship) for technical contributions to the neutronics simulations in this work. 


\section{Introduction}

The Simulation-based High-efficiency Advanced Reactor Prototyping (SHARP) toolkit [Mahadevan 2014], [Merzari 2015] is under development by the Nuclear Energy Advanced Modeling and Simulation (NEAMS) Campaign of the U.S. Department of Energy, Office of Nuclear Energy. The SHARP toolkit is a high-fidelity reactor simulation tool comprised of the neutronics module PROTEUS [Shemon 2014], thermal hydraulics module Nek5000 [Fischer 2008], and structural mechanics module Diablo, and therefore the toolkit can analyze multi-physics thermal-structural-neutronics phenomena of core configurations with complex geometric features.

After releasing the SHARP toolkit in 2016, the main focus of the Reactor Product Line (RPL) of the NEAMS Campaign was shifted toward testing, validation and verification of SHARP component codes. To better understand and exploit the benefits of advanced modeling simulations to improve fast reactor economics, the NEAMS Campaign initiated the "Sodium-Cooled Fast Reactor (SFR) Challenge Problems" task whose primary objective is to demonstrate the value of the SHARP toolkit to important problems relevant to SFRs.

The first challenge problem is the assessment of hot channel factors (HCFs) for SFR designs. The primary objective of this challenge problem is to demonstrate the reduction or elimination of uncertainties that are involved in the SFR designs, and to assess the impact on fast reactor economics. For a particular design parameter such as fuel centerline temperature, the HCF is defined by comparing the maximum temperature under nominal and perturbed conditions. The temperature differences between the nominal and perturbed conditions are due to uncertainties that are involved in the nominal value calculations: i.e., if a $\mathrm{HCF}$ is greater than unity for a particular parameter, the decimal part (i.e., HCF minus one) represents uncertainties.

In the United States, sets of HCFs were developed for the Fast Flux Test Facility (FFTF), Clinch River Breeder Reactor (CRBR), and Experiment Breeder Reactor II (EBR-II). No additional attempts to calculate updated HCFs have been made since the SFR development programs were canceled in the mid-1990s. Looking back at the earlier efforts on the HCF development for FFTF, CRBR, and EBR-II, limited computing power and approximations in modeling and simulation methods resulted in the prediction of neutronics and thermal-hydraulic parameters with relatively large uncertainties, where some uncertainties could only be quantified by expensive experiments. The high-fidelity and multi-physics capabilities of the SHARP toolkit, accompanied by remarkable progress in the computing technologies during the last few decades, now make it possible to calculate most design parameters without the support of mockups or experiments and reduce uncertainties significantly compared to the uncertainties involved in the FFTF or CRBR designs. Reduction of the uncertainties (i.e., reduction of HCFs closer to unity) allows an increase in nominal parameters and safety margin, which in turn improves the economic competitiveness of the SFR. To demonstrate the economic benefits, an assessment of HCFs using the SHARP toolkit was initiated in this study. However, it is also recognized that the assessment of HCFs are challenging because of significant computational resources requirements. 
The second challenge problem is the assessment of the impact of the "SHARP Zoom" capability, which is a hybrid core modeling and simulation scheme enabled by combining coarse-andhomogeneous meshes and fine-and-heterogeneous meshes: i.e., most assemblies are represented using conventional homogenized meshes, but few assemblies of interest are represented with pinby-pin heterogeneous meshes in order to gain accurate local information. If the zoom capability is feasible and produces accurate results at selected local areas and integral quantities globally, the computational expense of using high fidelity SHARP toolkit will be significantly reduced, and improve the usability of SHARP toolkit. However, the implementation of the zoom capability is also challenging because the convergence of solutions and the resulting accuracy of the zooming calculations are dependent on the communication between the global coarse meshes and local fine meshes in multi-physics calculations.

The primary purpose of this study is to demonstrate the capability of the SHARP toolkit to resolve the two aforementioned challenge problems. For this study, the $100 \mathrm{MWe}$ Advanced Sodium-cooled Fast Reactor (AFR-100) [Grandy 2013] was selected as a reference SFR for evaluating HCFs and testing the feasibility of the SHARP zoom capability.

In Section 2, the two SFR challenge problems are discussed in detail, including potential benefits from using high fidelity tools to solve the problems. Information on the AFR-100, the SHARP toolkit, and the computation resources used in this study are provided in Section 3. In Sections 4 and 5, the results of this study on the two SFR challenge problems are discussed, respectively. Finally, the conclusion of this study is in Section 6. Various sensitivity calculations were performed in this study, and the results are presented in Appendices. 


\section{SFR Challenge Problems}

In this chapter, we discuss the two types of problems targeted in this study as applications for the SHARP toolkit: hot channel factor (HCF) calculations, and the "SHARP Zoom" capability for a sodium-cooled fast reactor design (SFR).

\subsection{Assessment of Hot Channel Factors}

Various uncertainties are involved in the predictions of reactor design parameters, which include theoretical and experimental analysis uncertainties, instrumentation uncertainties, manufacturing tolerances, correlation uncertainties, and method and simulation uncertainties. In order to assure integrity of fuel elements and reactor structures and ultimately to protect the health and safety of the public and environment, the fuel, cladding, and coolant temperatures should not exceed the design limits with minimum margins to design limits. For instance, in order to assure cladding integrity of a metallic fuel SFR, the peak cladding inner-wall temperature is required to be below the fuel-cladding eutectic formation temperature as shown in Figure 2.1.1.

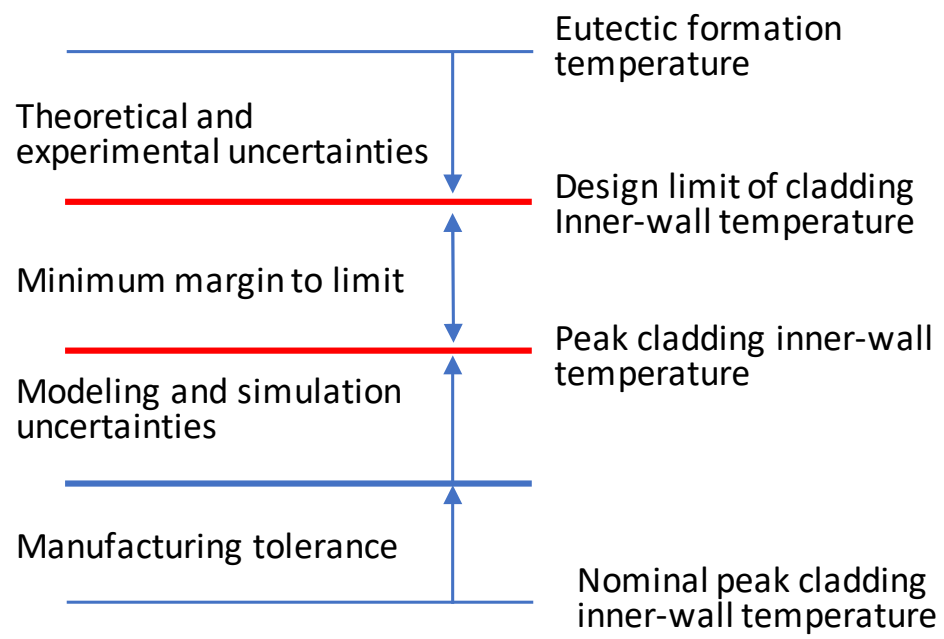

\section{Figure 2.1.1 Schematic of assessment of margin to fuel-cladding eutectic formation}

It is difficult to pin-point the exact eutectic formation temperature of a metallic fuel in an SFR because fuel-cladding eutectic formation is a complex phenomenon affected by fuel compositions, temperature, and irradiation histories. In order to account for theoretical and experimental uncertainties, the design limit (upper red line) of the eutectic formation temperature is lower than the value determined theoretically or by empirical corrections. On the other hand, the peak cladding temperature (lower red line) increases from the nominal calculation to account for manufacturing tolerances and uncertainties of cladding thickness and compositions. After considering all uncertainties (including manufacturing tolerances), the difference between the design limit and the 
peak cladding inner-wall temperature (gap between two red-lines in Figure 2.1.1) is defined as the "minimum margin to limit".

In Figure 2.1.1, the gap from the nominal peak cladding inner-wall temperature to the eutectic formation temperature minus the minimum margin to limit denotes the uncertainties that are involved in the calculation of the design parameters, and in order to count the uncertainties in the reactor design, so-called hot channel factor (HCF) methods have been developed and utilized since the early days of civil nuclear plant development. The hot channel factors are dimensionless numbers greater than unity such that the decimal part (i.e., HCF minus one) represents uncertainties in the parameter. Many ways to define the HCFs have been developed, but the semi-statistical method [Carelli 1980], which consists of direct and statistical hot channel factors, was adopted in this work.

In the United States, the SFR HCFs have been developed under the various SFR development programs, and the magnitudes of HCFs were strongly related to the status of modeling and simulation and computing power when the HCFs were developed. Figure 2.1.2 shows the SFR development, deployment, and operation history, including the activities on HCF development aimed at specific SFRs.

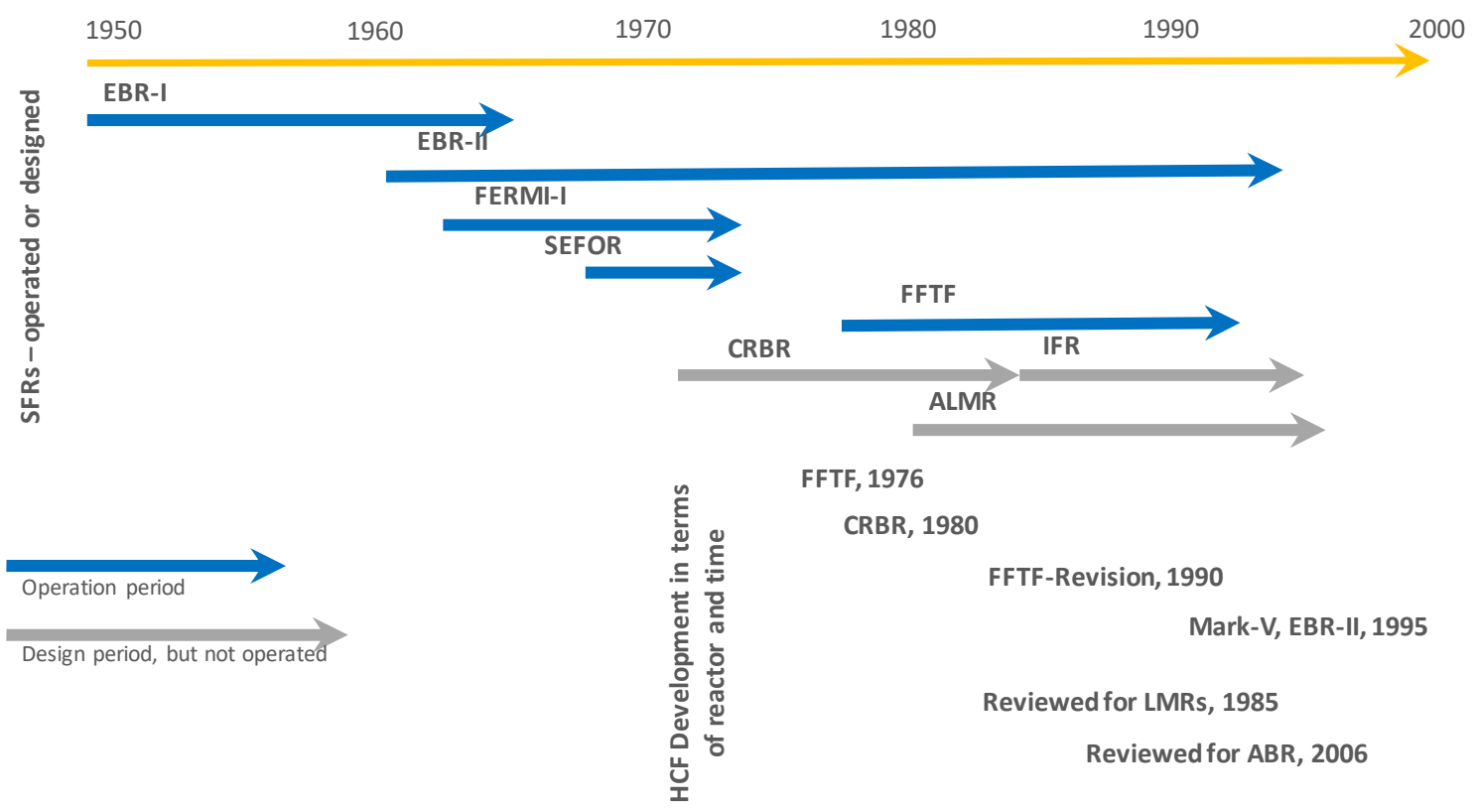

Figure 2.1.2 SFRs operated or designed in the United States

Four sets of HCFs have been developed under SFR programs in the United States. A set of HCFs was developed for FFTF initially in 1976 and later updated in 1990. A significant effort to develop HCFs was conducted to support the licensing of Clinch River Breeder Reactor (CRBR) in 1980. 
The last set of HCFs was developed in 1995 under the Integral Fast Reactor (IFR) project to develop Mark-V metallic fuels in the EBR-II. However, the EBR-II and FFRF were permanently shut down, and SFR programs were canceled in the mid-1990s, and therefore no additional efforts were regarding HCFs since then, except for reviews of existing HCF sets for new SFRs.

The HCFs of the CRBR [Carelli 1980] and EBR-II [Ku 1994] related to the cladding inner-wall temperature are compared in Table 2.1.1. The set of HCFs for the CRBR was developed in 1980, while the set for the EBR-II was developed in 1995. During the 15-year time-lag, number of HCFs was significantly reduced and the magnitudes of several HCFs were also changed. This trend informs that several uncertainties were eliminated or changed in the later design even though those uncertainties were involved in the earlier designs. The same trend is also observed when comparing two sets of HCFs for FFTF developed in 1976 and 1990.

Table 2.1.1 Comparison of HCFs related to cladding mid-wall temperature

\begin{tabular}{|c|c|c|c|c|c|c|}
\hline & \multicolumn{3}{|c|}{ CRBR - 1980} & \multicolumn{3}{|c|}{ EBR-II - 1995} \\
\hline & cool & film & clad & cool & film & clad \\
\hline \multicolumn{7}{|l|}{ Direct hot channel factor } \\
\hline Power measurement & 1.03 & 1.03 & 1.03 & 1.02 & 1.02 & 1.02 \\
\hline Inlet flow maldistribution & 1.02 & 1.006 & & & & \\
\hline Flow distribution calculated & 1.03 & & & & & \\
\hline Assembly flow maldistribution & 1.08 & & & & & \\
\hline Cladding circumferential temp. & & $1.7-2.1$ & 0.8 & & & \\
\hline Pellet-cladding eccentricity & & 1.14 & 1.14 & & & \\
\hline \multicolumn{7}{|l|}{ Statistic hot channel factor (3-sigma) } \\
\hline Reactor $\Delta \mathrm{T}$ variation & $\sim 1.14$ & 1.016 & & & & \\
\hline Inlet flow maldistribution & 1.059 & & & 1.079 & 1.025 & \\
\hline Wire Wrap orientation & 1.01 & & & 1.01 & & \\
\hline Sub-channel glow Area & 1.019 & & & & & \\
\hline Film Heat Transfer Coefficient & & 1.12 & & & 1.20 & \\
\hline Pellet-cladding eccentricity & & $1.0-1.17$ & $1.0-1.17$ & & & \\
\hline Coolant Properties & 1.017 & & & 1.06 & & \\
\hline Fissile Maldistribution & 1.058 & 1.005 & & 1.06 & 1.06 & 1.06 \\
\hline Nuclear data & & & & 1.07 & 1.10 & 1.10 \\
\hline Cladding thickness and conductivity & & & 1.12 & & & 1.085 \\
\hline Intra-assembly flow maldistribution & 1.058 & 1.005 & & 1.056 & 1.018 & \\
\hline
\end{tabular}

The time evolution of available computing power (in particular, memory and computation speed) and modeling and simulation tools used for development of HCFs are shown in Figure 2.1.3. Early HCF development took place in the 1970's and 1980's. In the 1980's and 1990's, the computation codes currently used for SFR design were developed and refined. Post 2010, the SHARP toolkits that are under development in the NEAMS campaign are seen.

Looking back at the earlier efforts for the FFTF and CRBR during the 1970s and early 1980s, limited computing power and numerous approximations in modeling and simulation methods 
resulted in the prediction of neutronics and thermal-hydraulic parameters with relatively large uncertainties, where some uncertainties could only be quantified by expensive experiments. For instance, the neutronics codes (such as XSRES-W1DX, DOT(XY), W-2DB, etc.) used for HCFs developments solved few group, low-dimension (1D, 2D, or 1D/2D synthesis) diffusion equations in a homogeneous medium [Hardie 1969, Mynatt 1973, Little 1969], and in order to compensate for the simplifications and approximations, the resulting neutron fluxes and integral parameters (for instance, core multiplication factor, reactivity coefficients, kinetics parameters) were adjusted by mockups performed in zero power critical experiment reactors (ZPR, ZPPR). Similarly, other codes also solve simplified system equations using empirical correlations and approximations in simplified or approximated geometries. For instance, the thermal-hydraulic code such as the early version of COBRA and ENERGY solved single or multi-channel equations with approximated wire-wrap models, and the results were calibrated using the experiments [Yang 2005].

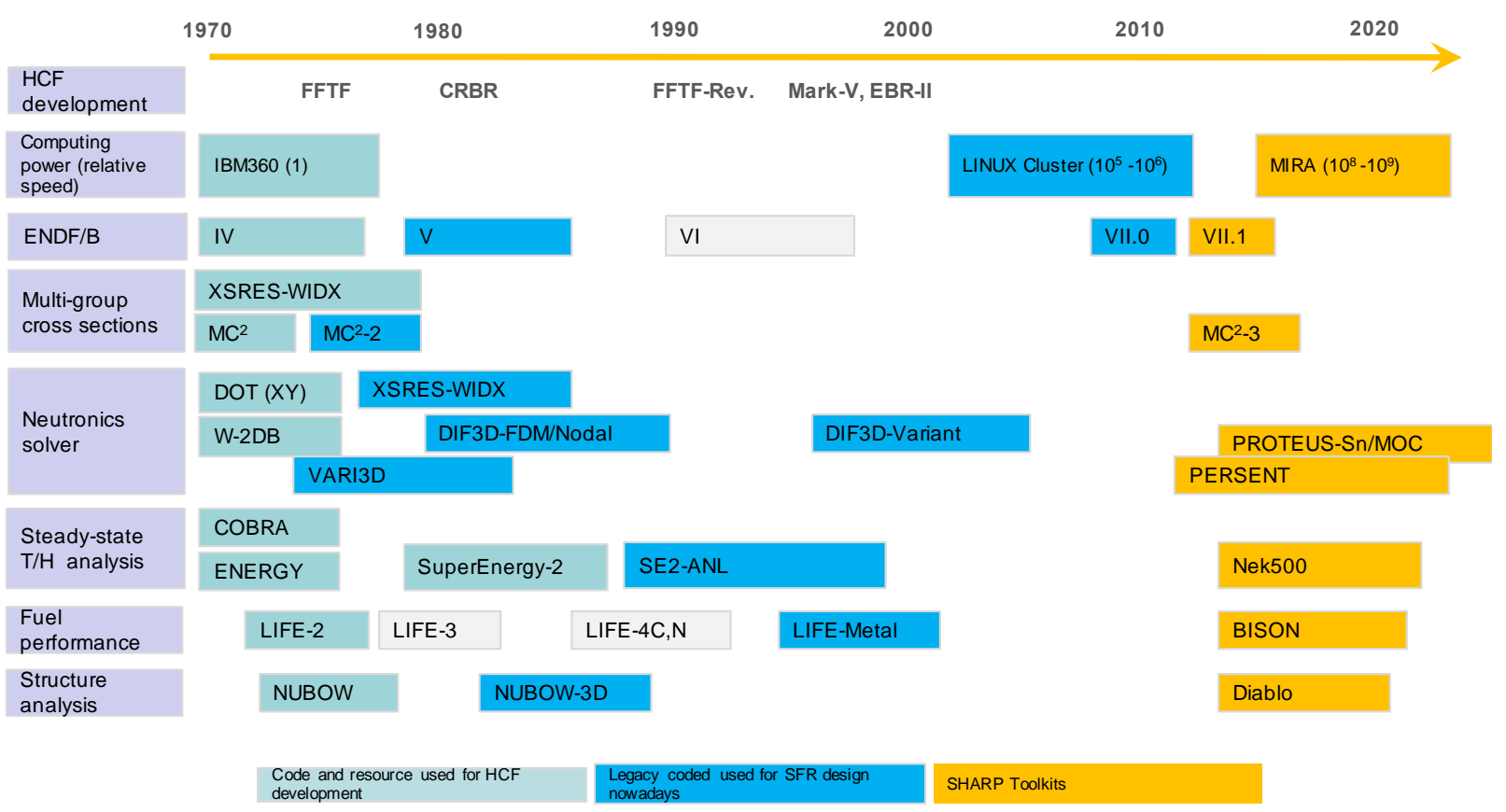

Figure 2.1.3 Evolution of computational resources and codes

During the last few decades, there were brilliant progresses in the computing power and computation code systems which enabled much more advanced and accurate modeling and simulation. For instance, the Mira BlueGene/Q supercomputer, one of the world's leading supercomputers, can do in one day what would take more than 1000 years using the IBM360 computer used in 1970s and 1980s, and today's high-fidelity multi-physics SHARP toolkit can solve the neutron transport and computational fluid dynamics equations without major physics approximations on the full geometry. Thus, the uncertainties associated with HCFs in the early SFR designs can be reduced or somehow eliminated using the high-fidelity multi-physics SHARP toolkit on high performance machines like Mira. 
Based on the observations and lessons learned from the SFR development programs in the United States, following two questions related to the HCFs are raised as the motivations of this study:

1. Can the SHARP toolkits calculate hot channel factors without extra mockup or experiments?

2. Can uncertainties that have been involved in the earlier SFR designs be eliminated or reduced using the SHARP toolkit and current computation resources?

If the answers for both questions are "yes", there are great economic benefits to SFR development and deployment. The first question is related to the overall development cost of SFRs. As explained, mockups and experiments have been performed in the earlier SFR development programs to compensate for the simplifications and approximations involved in the reactor designs. However, most legacy critical and experimental facilities have been decommissioned, and new construction of or recovering experimental facilities would be extraordinarily expensive or not allowed due to lack of fissile materials. Thus, if the SHARP toolkits can calculate HCFs reliably without mockups or experimental supports, overall development cost of future SFRs will be lowered significantly.

The second question is also related to reactor economic benefits and reliable operations because the reduction or elimination of uncertainties allows an increase in the peak cladding inner wall temperature, which ensure reliable operations with additional margin to design limits or/and permits extra increase in the reactor power rate. To emphasize the impact of HCFs reduction or elimination, the peak inner wall temperatures were evaluated using two sets of HCFs, and results are illustrated in Figure 2.1.4. The nominal values were arbitrarily selected from conventional SFR designs, the CRBR HCFs are obtained from Table 2.1.1, and the artificially improved HCFs were reduced by a factor of two from the CRBR HCFs.

The peak cladding inner temperature increases to $623{ }^{\circ} \mathrm{C}$ from the nominal temperature of 581 ${ }^{\circ} \mathrm{C}$ when accounting for the uncertainties considered in the CRBR design. However, if the HCFs decreased by a factor of two, the peak cladding inner-wall temperature decreases to $602{ }^{\circ} \mathrm{C}$. The reduction of cladding temperature of $21{ }^{\circ} \mathrm{C}$ would allow an increase in power rate: i.e., by assuming that other design parameters are also reduced with the same manner, the reactor power rate can be increased by $\sim 12 \%$, which yields extra earnings of $\sim \$ 100 \mathrm{M}$ per year from a $1000 \mathrm{MWe}$ fast reactor (based on current electricity cost of 12 cents per $\mathrm{kWh}$ ). 


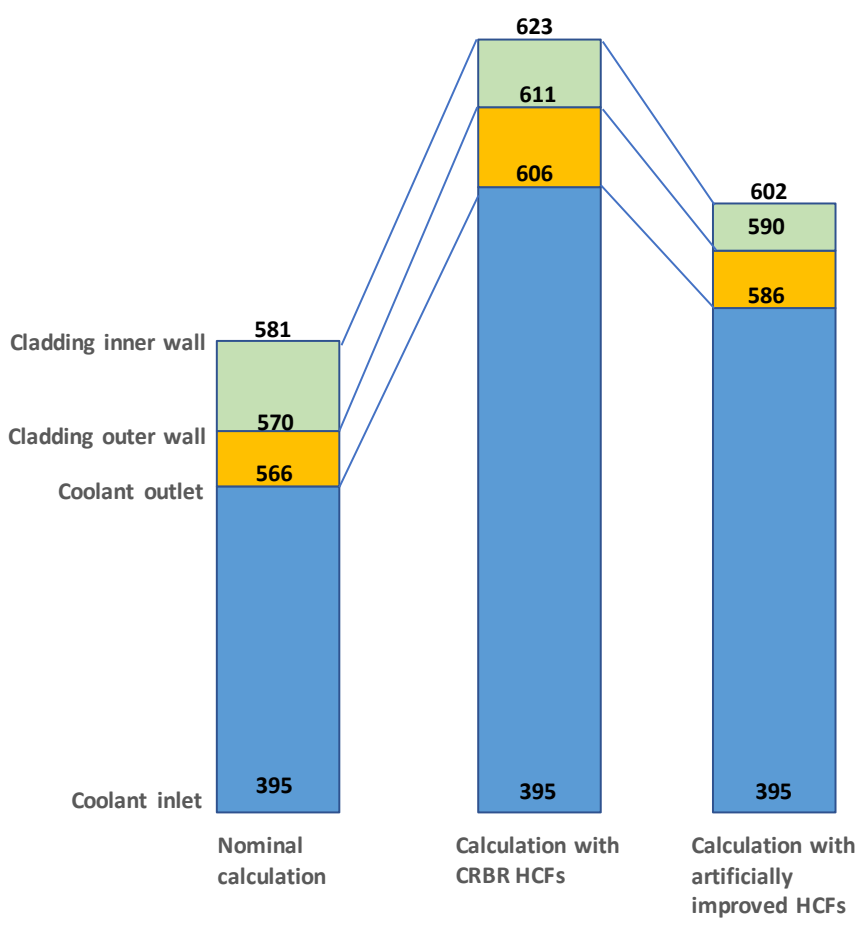

Figure 2.1.4 Peak cladding inner wall temperature depending on HCFs (artificial HCFs were hypothetically reduced from the CRBR HCFs)

Therefore, the reduction or elimination of uncertainties, and the calculations of HCFs without mockups or experimental supports will enhance the safety margins or/and improve the economic competitiveness of a SFR, and these benefits are the motivation of this study. As a tradeoff, however, the assessment of HCFs is challenging because high-fidelity computation tools (such as the SHARP toolkit) and high-performance computing power are required.

In this effort, the 100 MWe Advanced Sodium-cooled Fast Reactor (AFR-100) [Grandy 2013], DOE's non-water cooled small modular fast reactor, is selected as the reference fast reactor concept, and its neutron flux, power, and temperature distributions will be evaluated using the highfidelity multiphysics tools PROTEUS (neutronics) and Nek5000 (CFD) codes which are physics modules in the DOE NEAMS SHARP Toolkit for multi-physics reactor analysis. Specifically, hot channel factors will be calculated for a variety of perturbations in the AFR-100 design.

\subsection{SHARP Zoom Capability}

The SHARP toolkit produces high-fidelity solutions on high-performance computing platforms for core configurations with complex geometric features. However, whole core, multi-physics calculations using the SHARP toolkit are still impractical for large size problems because of excessive computational resources and time required until the solutions are fully converged in the iterative multi-physics calculations between the sub-component modules of the SHARP toolkit. To address this concern, the "SHARP Zoom" concept was proposed. 
The SHARP zoom concept is a hybrid core modeling and simulation scheme whereby coarseand-homogeneous meshes and fine-and-heterogeneous meshes are combined: i.e., most assemblies are represented using the conventional homogenized meshes, but a few assemblies of interest are represented with pin-by-pin heterogeneous meshes in order to gain accurate local information.

This zooming concept is useful to produce bounding values that are needed for development of licensing documents or a specific fuel development. For instance, in order to assure the integrity of fuel elements, the bounding values such as peak cladding inner-wall temperature, peak fuel centerline temperature, peak discharge burnup, peak linear power density, etc., are needed rather than the integral or average values. For development of a specific fuel concept, local irradiation information is needed rather than the integral information. Figure 2.2.1 shows the Americiumbearing blanket $(\mathrm{AmBB})$ concept that is under development by CEA, France for minor actinide transmutation using a SFR. The recovered Americium from existing reactors and the external uranium are mixed together and loaded into radial blanket regions for burning minor actinides. For this case, by adopting the zooming concept (i.e., pin-by-pin modeling of AmBB assembly and homogeneous modeling of other assemblies), the SHARP toolkit can provide detailed information (such as flux, temperature, burnup) needed for irradiation tests of AmBB fuel pins.

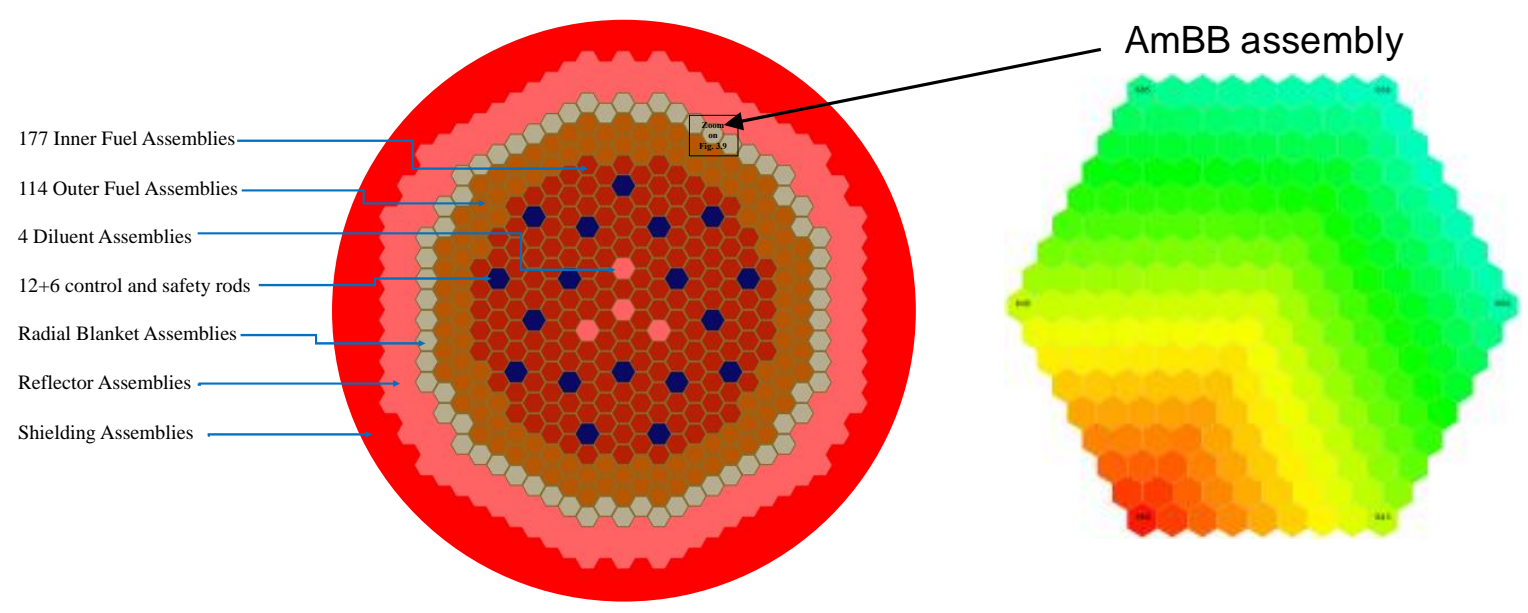

Figure 2.2.1 Americium-bearing blanket (AmBB) concept

The feasibility of the SHARP zoom concept was tested by comparing detailed pin-by-pin power and temperature distributions in the interested local areas with results obtained from conventional legacy code calculations. The SHARP zoom concept is tested in this work for a fuel assembly in the outer fuel zone of the AFR-100 design, which is an assembly surrounded by a mixture of other fuel assemblies. Neutronics zooming in this case is accurate and dramatically reduces the computational expense of the simulation. Additionally, the multi-physics coupling is necessary only for the focal assembly, reducing the computational expense of the fluid dynamics modeling dramatically. Therefore, by zooming into selected regions of interest, the cost of using the high fidelity SHARP toolkit is significantly reduced, and whole core effects can be taken into account 
with high detail in focus regions using accessible computational resources. This greatly improves the usability of the SHARP toolkit. . 


\section{SFR Design Problems and Computational Tools}

In this study, the 100 MWe Advanced Fast Reactor (AFR-100) [Grandy 2013] was selected as the reference core to resolve the SFR challenge, and the PROTEUS and Nek5000 codes were selected as the neutronics and steady-state thermal-hydraulics analyses, respectively. In this section, information on AFR-100, the SHARP toolkit, and the available computational resources are described.

\subsection{Advanced Fast Reactor as SFR Design Problem}

The AFR-100 was developed to target emerging markets where a clean, secured and stable electricity resource is required but a large-scale plant is not accommodated. The AFR is aimed to be sized for local small grids, transportable from pre-licensed factories to the remote plant site for affordable supply, and able to operate for a long period without frequent refueling. To achieve these strategic goals, several design requirements have been proposed for the AFR core development; a power rating of $100 \mathrm{MWe}$ which is equivalent to $250 \mathrm{MWth}$ with the target thermal efficiency of $40 \%$, a long refueling interval with small burnup reactivity swing, a core barrel diameter of less than $3.0 \mathrm{~m}$, and an active core height of less than $1.5 \mathrm{~m}$.

Detailed information on the AFR-100 is provided in Appendix A, including the computation methods. Figures 3.1.1 and 3.1.2 show the radial and axial core layouts of the AFR-100 core, respectively, and the major core performance parameters are summarized in Table 3.1.1.

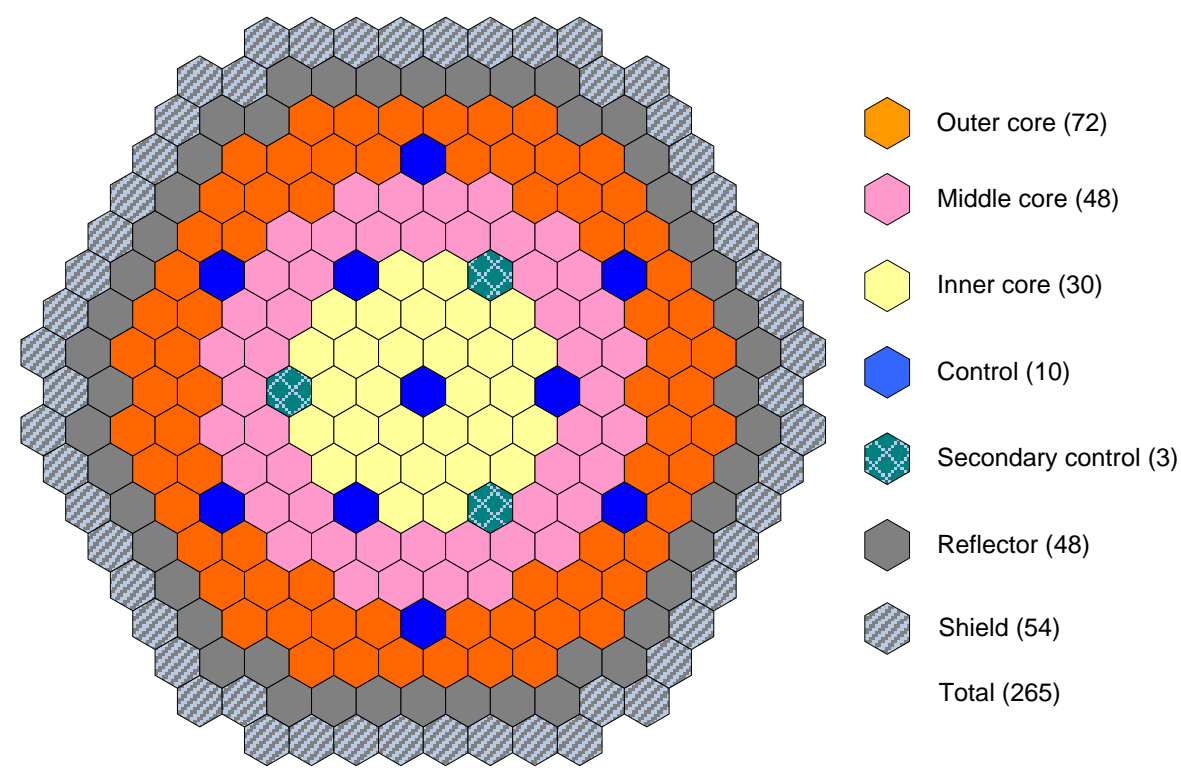

Figure 3.1.1 Core radial layout of AFR-100 


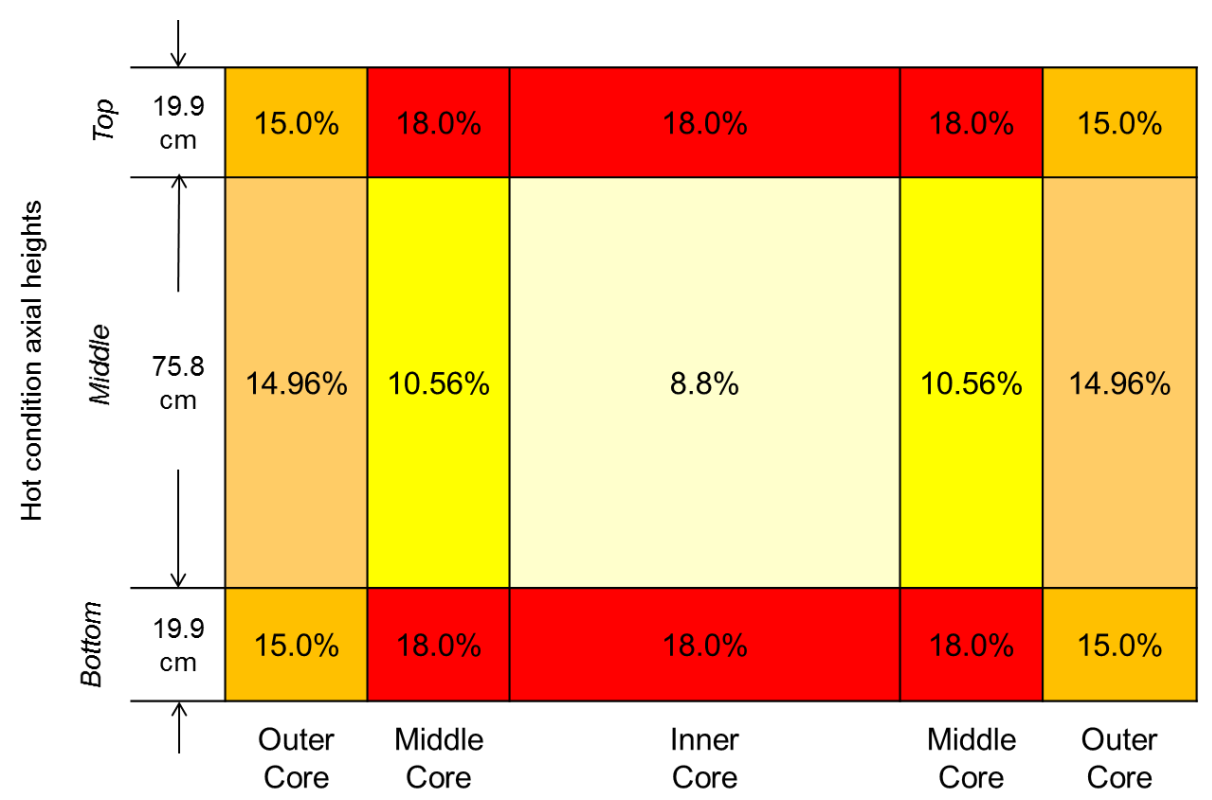

Figure 3.1.2 Core axial layout and uranium enrichment distribution

Table 3.1.1 Core performance parameters

\begin{tabular}{|l|c|}
\hline Parameters & AFR-100 \\
\hline Thermal power, MWt & 250 \\
\hline Refueling interval, year & 30 \\
\hline Number of batches & 1 \\
\hline Fuel form & $\mathrm{U}-\mathrm{Zr}$ \\
\hline Active core height, cm & 110 \\
\hline Average power density, $\mathrm{kW} / \mathrm{l}$ & 64.3 \\
\hline Fuel pin overall length, cm & 222 \\
\hline Initial heavy metal loading, $\mathrm{t}$ & 23.9 \\
\hline Specific power density, MW/t & 10.5 \\
\hline Average enrichment, \% & 13.7 \\
\hline Burnup reactivity swing, \% $\Delta \mathrm{k}$ & 1.1 \\
\hline Overall fissile breeding Ratio & 0.80 \\
\hline Average discharge burnup, MWd/kg & 101 \\
\hline Peak discharge burnup, MWd/kg & 172 \\
\hline Peak fast fluence, $10^{23} \mathrm{n} / \mathrm{cm}^{2}$ & 5.97 \\
\hline Linear heat rate (average/peak), kW/, & $15.2 / 28.2$ \\
\hline
\end{tabular}

The AFR-100 core adopted an onion-type enrichment zoning concept that was developed for a long refueling scheme and flat radial power distribution. The active core is divided into three different enrichment zones radially, and each zone has multiple zones axially. The average uranium enrichment is $11.97 \%$ for the inner core, $13.12 \%$ for the middle core, and $14.97 \%$ for the outer core. 
The core can maintain criticality for 30 years by employing the unique enrichment zoning strategy of the AFR-100 with low reactivity swing during the reactor life time. The average discharge burnup is $101 \mathrm{MWd} / \mathrm{kg}$ and the peak discharge burnup is $172 \mathrm{MWd} / \mathrm{kg}$.

\subsection{Computational Tools and Resources}

The SHARP Toolkit is a DOE NEAMS software product that performs highly detailed multiphysics calculations with online data transfer. In this section, two sub-components of the SHARP toolkit, PROTEUS and Nek5000, that were used to resolve the SFR challenge problems are briefly described, including the overall computation approach by coupling two component codes and computation resources.

\subsubsection{PROTEUS Neutronics Code}

PROTEUS has two neutronics solvers, PROTEUS-SN and PROTEUS-MOCEX, and both solvers were used for this work. All published scaling studies were performed with the PROTEUSSN code which we report below.

The PROTEUS-SN solver was a previous Gordon Bell Finalist for computational performance on the BlueGene/P (BG/P) architecture. It solves the second order, even parity form of the neutron transport equation using discrete ordinates approximation in angle, unstructured continuous finite element method in space, and multigroup approximation in energy. The code relies on the PETSc linear solver package for performing large scale matrix solves of the underlying eigenvalue problem, the METIS package for online mesh partitioning, and the HDF5 package for detailed output. Parallelism is performed with MPI, and depending on the memory requirements for the cross section data, typically 1 MPI rank/core is optimal and reasonable. The code does not use multithreading at this point as threading is not particularly useful for the matrix operations in this case.

Scaling data is provided for the $\mathrm{BQ} / \mathrm{P}$ architecture for a fast reactor problem in Tables 3.2.1.1 and 3.2.1.2 (all data accumulated at 1 MPI rank per core). Performance is very similar on the BlueGene/Q architecture provided the parallelization regime of $>1500$ spatial vertices/process and $>1-2$ angles per process is followed. The lower memory per core on BG/Q does not impact the simulations as long as parallelization in space and angle are maximized and the number of energy groups is less than 70 . In cases with 70 or more energy groups, 8 MPI ranks per node or less are often required to fit the cross section data into memory.

The PROTEUS-MOCEX code solves the method of characteristics equations in the 2D plane for an unstructured mesh and solves the exact equations in the axial plane using the discontinuous Galerkin finite element method. The method is also known as "extruded MOC" as it relies on an extruded 2D mesh in the axial plane for geometry description. The method is more accurate than other extruded MOC methods such as DeCart and MPACT which rely on the 2D/1D synthesis method and can suffer from convergence and accuracy issues in certain core geometries. While we do not have detailed scaling data to present for PROTEUS-MOCEX due to its recent development, 
it has performed well on both small, medium, and large clusters including the BlueGene/Q supercomputer. It is generally more memory intensive than the PROTEUS-SN code but uses less cores and less compute time, typically, to solve the same problem.

Table 3.2.1.1 Strong spatial scalability of PROTEUS-SN on BlueGene/P (ANL)

\begin{tabular}{|c|c|c|c|}
\hline Total Cores & Vertices Per Process & Total Time $(\mathrm{s})$ & Strong Scaling \\
\hline 8,192 & 7,324 & 2,402 & $100 \%$ \\
\hline 16,384 & 3,662 & 1,312 & $92 \%$ \\
\hline 24,576 & 2,441 & 873 & $92 \%$ \\
\hline 32,768 & 1,831 & 637 & $94 \%$ \\
\hline
\end{tabular}

Table 3.2.1.2. Weak angle scalability of PROTEUS-SN on BlueGene/P (ANL, JSC)

\begin{tabular}{|l|l|l|l|}
\hline Total Cores & Angular Directions & Total Time (s) & Weak Scaling \\
\hline 32,768 & 32 & 579 & $100 \%$ \\
\hline 73,728 & 72 & 572 & $101 \%$ \\
\hline 131,072 & 128 & 581 & $100 \%$ \\
\hline 163,840 & 160 & 691 & $84 \%$ \\
\hline 294,912 & 288 & 763 & $76 \%$ \\
\hline
\end{tabular}

Both PROTEUS-SN and PROTEUS-MOCEX have been studied for benchmark problems and compared well to Monte Carlo (MCNP or SERPENT) models as well as some limited validation data (ZPR, TREAT). However, the accuracy of high fidelity deterministic codes such as these is highly dependent on the accuracy of the multigroup constants fed into the codes as inputs. Significant research has been performed to assess the procedures for generating multigroup cross section data (an input parameter to deterministic neutronics codes like PROTEUS-SN and PROTEUS-MOCEX) for explicit pin-by-pin geometries [Hader 2014], [Jarrett 2016]. The techniques learned in the past few years have been implemented in this work.

\subsubsection{Nek5000 Thermal Hydraulics Code}

Nek5000 is an open source code designed to simulate unsteady incompressible Navier-Stokes flow, low Mach-number flows flow, heat transfer and species transport and incompressible magneto hydrodynamics (MHD). Nek5000 is a previous winner of the Gordon Bell Prize in high performance computing and continues to adapt to new architectures with its highly scalable algorithms. Its fast scalable multigrid solver can scale to more than 290,000 processors on the BG/P architecture using pure MPI with multiple ranks per core, and beyond 1M MPI ranks on BG/Q architecture at $60 \%$ efficiency with only 2000 DOFs/rank.

Nek5000 is based on a high-order spectral element method and achieves extremely rapid (exponential) convergence at low cost. Figure 3.2.2.1 shows the comparison of convergence behavior between Nek5000 and OpenFOAM (another well-known open source CFD code), where 
Nek5000 has a remarkable advantage on convergence behavior (fewer DOF are required for a desired accuracy). In addition to its high-order foundation, Nek5000 has several other features that make it ideally suited for large-scale parallel simulations. Temporal discretization is based on a high-order splitting that is third order accurate in time and reduces the coupled velocity-pressure Stokes problem to independent three Helmholtz and one elliptic solver per time step, i.e. for each velocity component and pressure, respectively. The velocity problems are diagonally dominant and thus easily solved by using Jacobi preconditioned conjugate gradient iteration. The pressure substep requires a Poisson solve at each step, which is performed through multigrid-preconditioned GMRES iteration coupled with temporal projection to find an optimal initial guess. Particularly important components of Nek5000 are its scalable coarse-grid solvers that are central to parallel multigrid. Nek5000 has been extensively tested for over 25 years.

More than 150 journal articles have been published by worldwide users of Nek5000. More than 400 tests have been performed to validate the models in the code in different research fields. The user community includes over 400 scientists and engineers in academia, laboratories and industry. The Nek5000 has been used for numerous INCITE and ALCC awards at the ALCF in recent years. All required physics modules for this work are already included in Nek5000. Figure 3.2.2.2 shows a schematic representation of the full hierarchy of thermal hydraulic codes in NEAMS toolkit. Given the computational cost, we focus on RANS-based CFD simulations for this SFR Grand Challenge program.

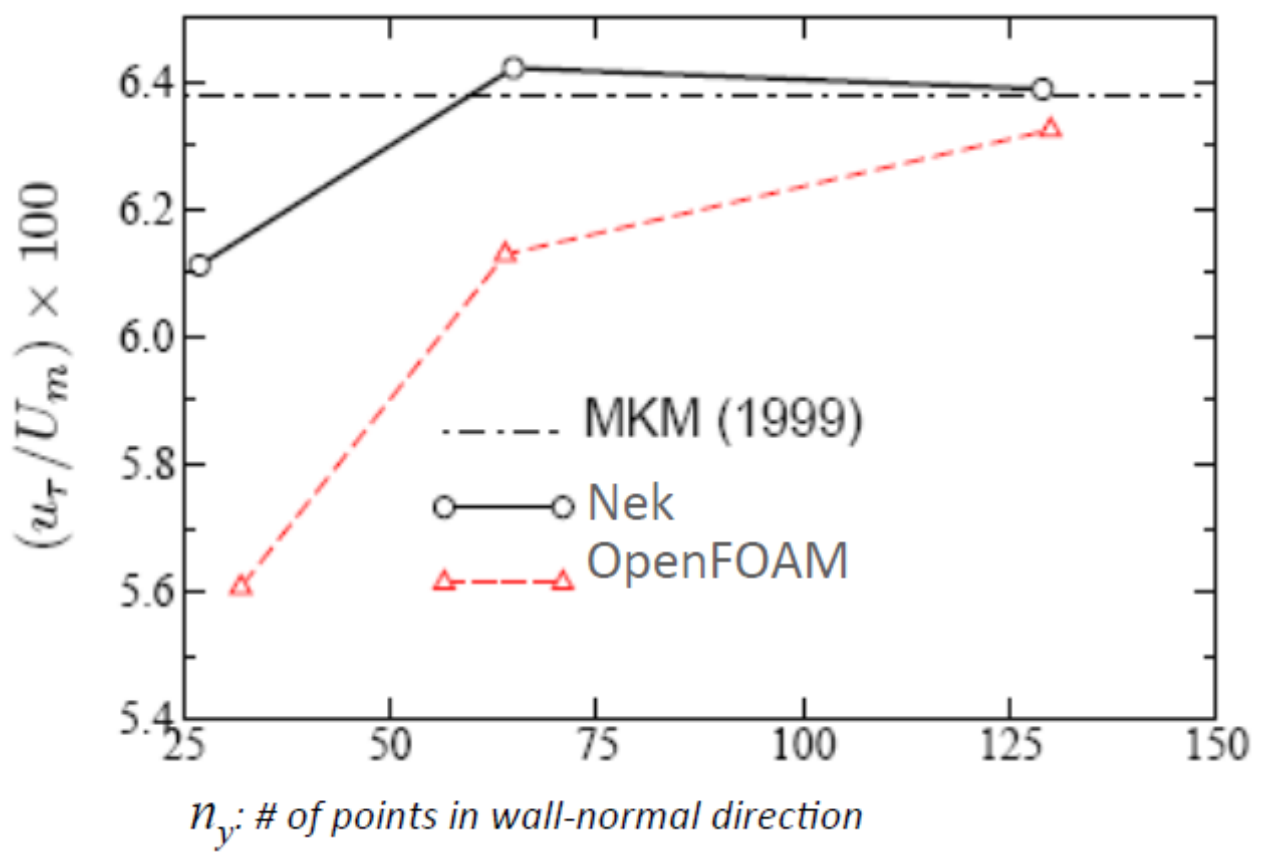

Figure 3.2.2.1 Comparison of convergence behavior between Nek5000 and OpenFOAM [Sprague 2010] 


\section{Deviation}

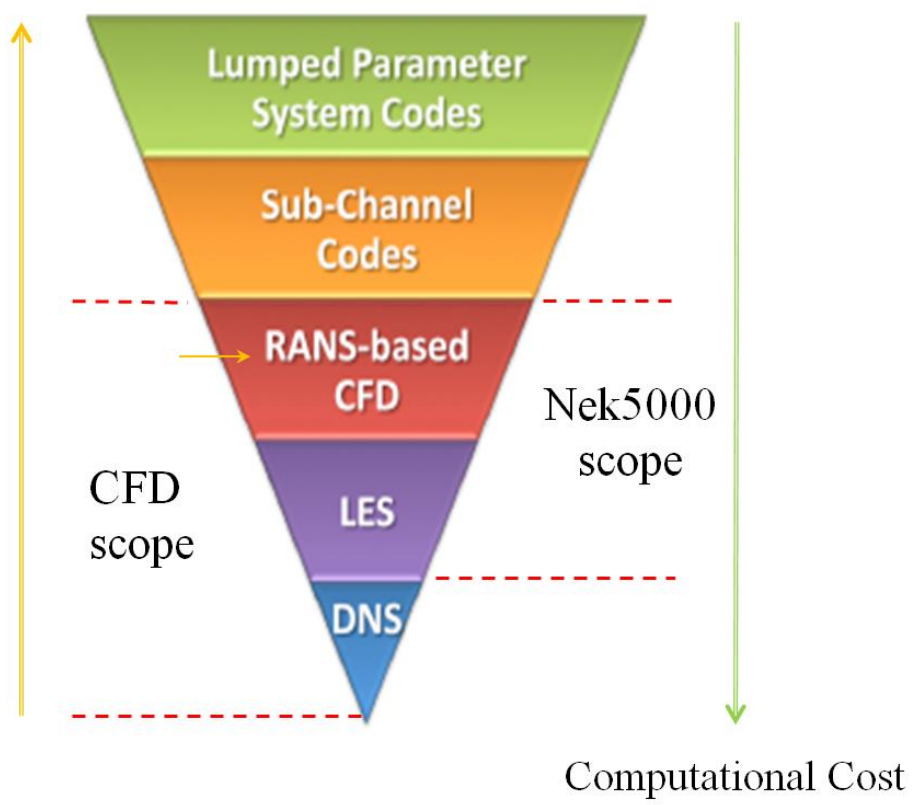

Figure 3.2.2.2 Hierarchical structure of NEAMS TH tools

\subsubsection{Multiphysics Coupling Approach and Computational Resources}

A schematic of the SHARP toolkit is shown in Figure 3.2.3.1. The PROTEUS neutronics solver shown in the figure is PROTEUS-SN (only one with ability to deform meshes) and Nek5000 fluid computational dynamics code are individual physics modules of the SHARP toolkit developed at Argonne National Laboratory under the DOE NEAMS program and other (Nek5000) funding. PROTEUS calculates the detailed power density and flux distributions in a nuclear reactor. Nek5000 calculates the detailed velocity and temperature distributions in a nuclear reactor core. PROTEUS and Nek5000 are tightly coupled together within the SHARP Toolkit and information (temperature, power) can be seamlessly exchanged online during the simulation while iterating between the two physics. We do not discuss the Diablo structural mechanics code in this work as it is not relevant for our applications. 


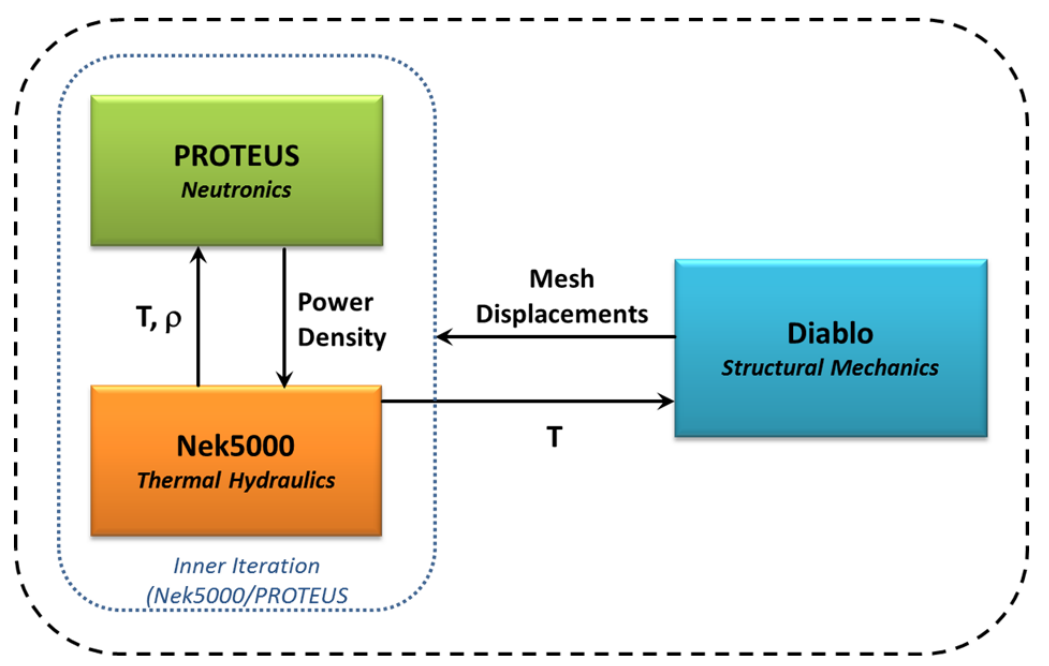

Figure 3.2.3.1 Schematic of online coupling using the SHARP toolkit

While online coupling within the SHARP toolkit certainly provides very accurate transfer of data (element wise basis), there are some limitations: (1) only one neutronics solver and one CFD solver are currently hooked up, (2) in today's implementation of SHARP, the codes must utilize the same number of compute cores during the simulation, and (3) parallelization in angle in PROTEUS within SHARP is prohibited, which means fewer cores total can be used and with a higher memory burden.

These limitations may need a large amount of computational resources for certain types of problems. Therefore, an offline coupling scheme was introduced in this study as shown in Figure 3.2.3.2 which allows a different neutronics solver (more appropriate for the proposed work) to be used along with Nek5000 and also allows each code to choose its own optimal parallelization scheme, providing much greater computational flexibility and efficiency. The drawback of offline coupling is the coarser-grained data transfer as well as the manual interventions that are required between code iterations that preclude efficient 2-way coupling.

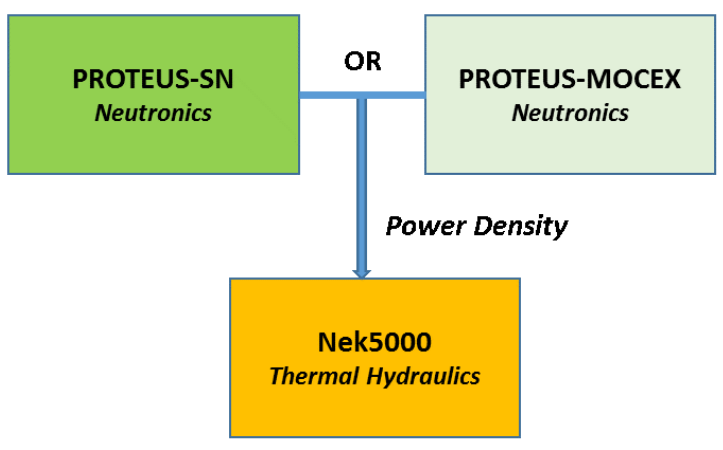

Figure 3.2.3.2 Schematic of offline coupling using components of the SHARP toolkit. 
There were two motivations to use offline coupling for this work. First, the PROTEUS-MOCEX solver was highly preferred due to performance issues of the PROTEUS-SN solver for the geometries chosen for the hot channel factor calculations. PROTEUS-MOCEX is an neutronics solver using an extruded method of characteristics methodology which is highly accurate for heterogeneous geometries. However, since PROTEUS-MOCEX is not hooked up with SHARP, it was necessary to perform coupling off-line. Secondly, the computational requirements of neutronics vs. $\mathrm{T} / \mathrm{H}$ for this work are vastly different and would require artificially large meshes to be used in order to maintain good computational scaling.

The offline coupling was performed one-way: initial calculation with PROTEUS-MOCEX, spatial distribution of power density given to Nek5000, and finally Nek5000 calculates temperature distribution. Ideally an iterative procedure would be implemented as shown in Figure 3.2.3.1, where neutronics would update the material cross sections based on temperature, and produce a new power density for Nek5000, but this would double the computational requirements and usually is not fully necessary due to quick convergence. In this work, the initial temperature distribution was assumed to be uniform (standard assumption) for convergence studies as well as for the initial power profiles given to Nek5000. Additionally, a relatively cheap STAR-CCM+ [CD-Adapco 2009] calculation was tentatively used to provide a better temperature guess and comparisons are performed against the uniform temperature distribution.

The available computational resources for this work include small local clusters (debugging), medium-scale high performance clusters (smaller production runs), and large-scale supercomputing machines (large production runs). Due to the high computational resource demands of some of the proposed challenge problems, two competitive proposals were written to access time on leadership and high-performance machines.

Local machines and small clusters are useful to perform pre-processing steps such as finite element mesh generation, multigroup cross section preparation, and testing of input files. No special proposals are needed to access these resources.

To perform more detailed testing, debugging, spatial and angular convergence studies, and small production runs for both PROTEUS and Nek5000, a pre-existing allocation at Argonne's Laboratory Computing Resource Center (LCRC) was utilized. Proposals are required for this resource and a proposal was submitted for FY18 in the amount of 450,000 core-hours. An existing allocation that was requested in FY16 was used for FY17 calculations. The LCRC is host to the Blues cluster (306 Sandy Bridge Xeon with 16 core/node and 64 GB, plus 40 Haswell Xeon nodes with 16 cores/node and $64 \mathrm{~GB}$ ) and Bebop cluster (664 Broadwall nodes with 36 cores/node and $128 \mathrm{~GB} /$ node, plus $352 \mathrm{KNL}$ nodes with 64 cores/node and $96 \mathrm{~GB}$ ). Roughly 150K-300K core hours are available per quarter, depending on user needs.

Finally, for the largest production simulations (in particular, PROTEUS heterogeneous neutronics simulations as well as Nek5000 wire wrap calculations), an Advanced Scientific Computing Research (ASCR) Leadership Computing Challenge (ALCC) proposal was written and 
awarded 44 million core-hours of compute time at the Argonne Leadership Computing Facility (ALCF), host to the Mira BlueGene/Q supercomputer. ALCF's Mira has 49,152 IBM BG/Q nodes with 16 cores/node and 16 GB of RAM per node. The ALCC award ran from July 1, 2017 through June 30, 2018. 


\section{Assessment of Hot Channel Factors}

The HCFs for the SFR were assessed and the results are presented in this Section. For this purpose, a literature review was performed and the HCFs that can be calculated with the SHARP toolkit were identified. A list of calculations to be performed as well as the neutronics and thermal hydraulics models and results for the hot channel factor calculations are discussed in this Section.

\subsection{Hot Channel Factors for Metallic Fuel SFRs}

The HCFs developed for the earlier SFRs have been reviewed in Appendix D, and the proposed set of HCFs for metallic fuel SFRs are listed in Table 4.1.1. In the last column, several HCFs are marked with "C" or "E" when the HCF can be calculated or eliminated by the SHARP toolkit, respectively. It is noted that two HCFs of "Intra-assembly flow maldistribution", and "Physics Modeling" can be eliminated in the HCF list if the uncertainties on modeling and simulations with the SHARP toolkit are assumed to be negligibly small.

Table 4.1.1 Proposed hot channel factors for metallic fuel SFR

\begin{tabular}{|c|c|c|}
\hline & Major source of uncertainties & $\begin{array}{c}\text { Remark } \\
(\mathrm{C} \text { or } \mathrm{E})^{\text {a) }}\end{array}$ \\
\hline \multicolumn{3}{|l|}{ Direct Hot Channel Factors } \\
\hline Power level measurement & $\begin{array}{l}\text { Few percent overpower is assumed in reactor normal } \\
\text { operation due to control rod dead-band }\end{array}$ & \\
\hline $\begin{array}{l}\text { Cladding circumferential } \\
\text { temperature }\end{array}$ & $\begin{array}{l}\text { Approximation in calculation of axial coolant } \\
\text { velocity and azimuthal temperature distribution } \\
\text { around a fuel pin. For instance, this HCF was } \\
\text { evaluated with a simplified wire-wrap model using } \\
\text { FATH0M-360 code and calibrated through ORNL- } \\
19 \text { tests. }\end{array}$ & $\mathrm{C}$ \\
\hline Control rod banking & $\begin{array}{l}\text { Control rods are grouped (banking) in operation } \\
\text { and there is manufacturing tolerance, which } \\
\text { introduces asymmetric power because insertion } \\
\text { depths are not identical. }\end{array}$ & \\
\hline \multicolumn{3}{|l|}{ Statistical Hot Channel Factors } \\
\hline $\begin{array}{l}\text { Reactor } \Delta \mathrm{T} \text { and inlet } \\
\text { temperature variation }\end{array}$ & $\begin{array}{l}\text { Uncertainties of reactor } \Delta \mathrm{T} \text { due to deterioration of } \\
\text { primary components. }\end{array}$ & \\
\hline Inlet flow maldistribution & $\begin{array}{l}\text { Uncertainties in inlet flow distribution due to flow } \\
\text { maldistribution in lower plenum, manufacturing } \\
\text { tolerances in internal structures and orifice, etc. }\end{array}$ & \\
\hline Loop temperature imbalance & $\begin{array}{l}\text { CRBR allows cold leg loop-to-loop temperature } \\
\text { imbalance of } 34{ }^{\circ} \mathrm{F} \text {, which results in } 4.9^{\circ} \mathrm{F}(2- \\
\text { sigma) uncertainty in inlet temperature. }\end{array}$ & \\
\hline Wire-wrap orientation & $\begin{array}{l}\text { Analyzed by sub-channel code, COTEC, which is } \\
1 \% \text { uncertainty. }\end{array}$ & $\mathrm{C}$ \\
\hline
\end{tabular}




\begin{tabular}{|l|l|c|}
\hline Sub-channel flow area & $\begin{array}{l}\text { Uncertainties from clad fabrication tolerance, } \\
\text { bowing, etc. }\end{array}$ & C \\
\hline Film heat transfer coefficient & Uncertainties from correlations. & C \\
\hline Cladding properties & $\begin{array}{l}\text { Uncertainties from manufacturing tolerance and } \\
\text { empirical correlations, etc. }\end{array}$ & C \\
\hline Coolant properties & $\begin{array}{l}\text { Uncertainties from material impurity and empirical } \\
\text { correlations etc. }\end{array}$ & C \\
\hline $\begin{array}{l}\text { Intra-assembly flow } \\
\text { maldistribution }\end{array}$ & $\begin{array}{l}\text { Uncertainties of flow distribution within assembly, } \\
\text { which are due to simplified model and applied } \\
\text { empirical factors of sub-channel codes. }\end{array}$ & E \\
\hline Physics Modeling & $\begin{array}{l}\text { There are many sub-factors under this category } \\
\text { such as nuclear data, flux solver approximation, 2D } \\
\text { synthesis method, etc. In FFTF and CRBR, lumped } \\
\text { uncertainties of power distribution were estimated } \\
\text { using ZPR, ZPPR mockups. }\end{array}$ & E \\
\hline Fissile fuel maldistribution & Manufacturing tolerance & C \\
\hline Fuel thermal conductivity & $\begin{array}{l}\text { Uncertainties due to fuel rod diameter, fresh and } \\
\text { irradiated fuel conductivity, porosity of swollen } \\
\text { fuel, redistribution, etc. }\end{array}$ & C \\
\hline Power level measurement & $\begin{array}{l}\text { Instrument uncertainties (flow rate, temperature, } \\
\text { etc.) and control systems }\end{array}$ & \\
\hline
\end{tabular}

a) $\mathrm{C}$ or E indicates that the HCF can be calculated (C) or eliminated (E) using SHARP toolkit.

\subsection{Strategy for Hot Channel Factor Assessments}

In this section, we describe the planned hot channel factor calculations for the beginning of cycle (BOC) AFR-100 fast reactor design, described in Appendix A. The vented fuel concept shown in Figure 4.2.1 was chosen for this analysis due to its shorter axial height compared to the non-vented concept.

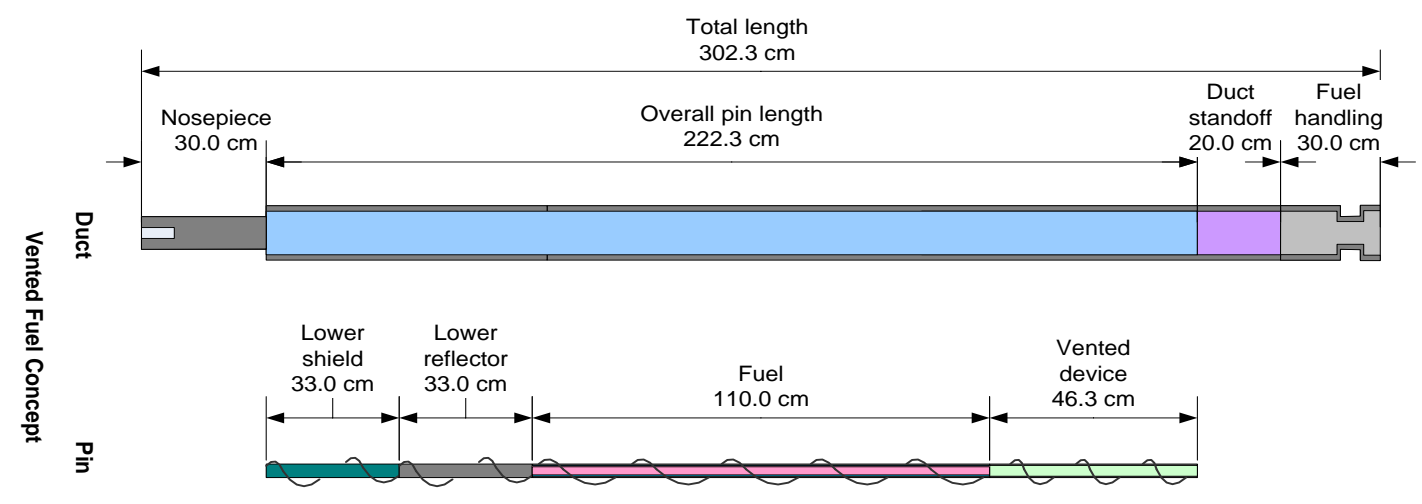

Figure 4.2.1 Depiction of fuel wrapped pin 
Each AFR-100 fuel assembly contains 91 helically wire-wrapped, closely packed fuel pins. In this study, in order to obtain accurate solutions using available computation resources within reasonable computation time, a wide-range of sensitivity studies were conducted to determine the required mesh discretization and other parameters for the PROTEUS and Nek5000 (see Appendix B and C). Sample meshes of a single fuel assembly with wire-wrap are shown in Figures 4.2.2. One can observe the extraordinary mesh complexity in the wire wrapped case. The wire-wrap around each fuel pin is the main source of strong inter-channel cross flow which is a significant energy transport mechanism in SFR assemblies. The wires have global influences on the flow behavior, and high speed patches of turbulence due to vortex shedding downstream of the wire persist for about a quarter of the wire-wrap periodic length as shown in Figure 4.2.3. Part of this work will assess the hot channel factor in bare bundle vs wire-wrap modeling.
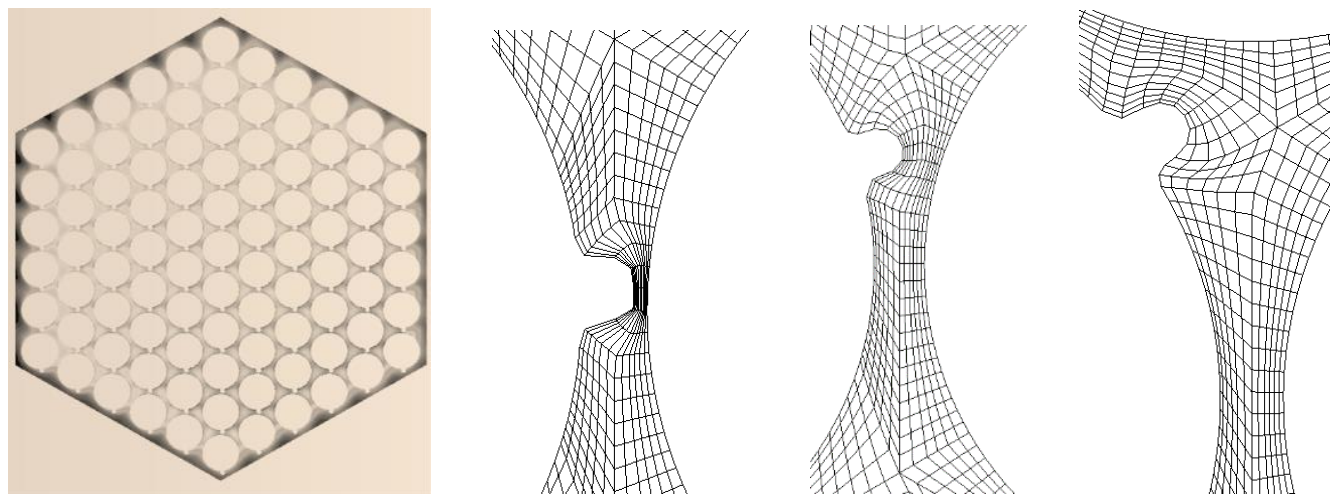

Figure 4.2.2 Close-up mesh of 91-Pin fuel assembly and varying sub-channel geometry (left: wire wrap, 3 right: Vicinity of wire wrap)

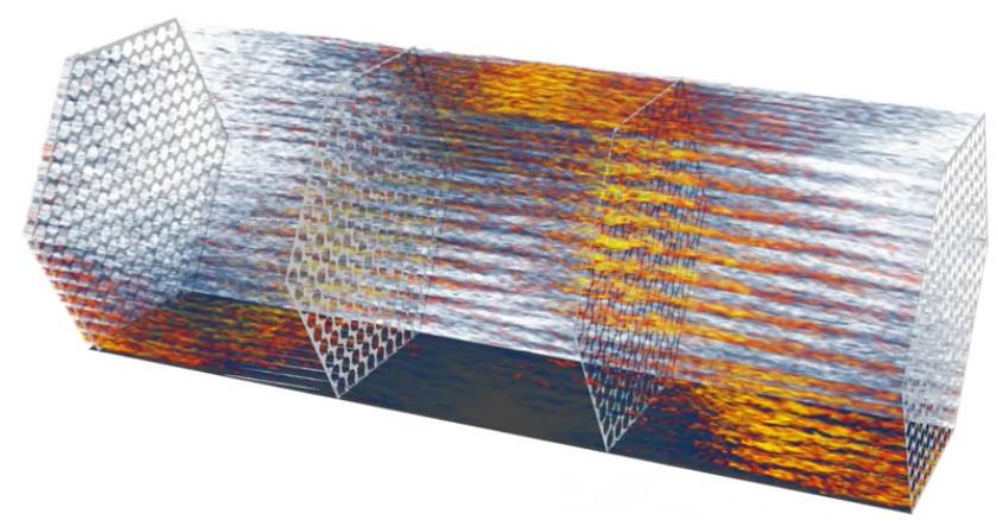

Figure 4.2.3 Nek5000 simulation for of 217-pin assembly with over 1 million grid points [Childs 2008]

It is noted that single pin or 7-pin bundles were utilized to calculate HCFs in the earlier SFR programs because of limited computation resources and approximations in computation tools, but 
the SHARP toolkit can model the full assembly explicitly. Among the HCFs listed in Table 4.1.1, some HCFs, in particular if manufacturing tolerances are associated, need stochastic simulations using 91-pin full assembly model. For instance, the HCF of fissile content maldistribution related to peak cladding inner-wall temperature should be calculated by randomly distributing fissile contents over 91 pins based on the manufacturing tolerance. However, a simplified 7-pin bundle model is sufficient rather than 91-pin full assembly model for some HCFs (such as coolant density uncertainty) because the uncertainties are uniformly smeared in the assembly. Thus, in this study, both a 7-pin bundle configuration and 91-pin full assembly configuration, depicted in Figure 4.2.4, were used for evaluating HCFs. In addition, since it is expected that the wire-wrap effect is small in some HCFs, both 7- and 91-pin configurations were modeled with and without wire-wrap. The two configurations are summarized here:

1. The 7-pin configuration is based on the AFR-100 inner fuel pin geometry (with and without wire wrap) surrounded by sodium coolant. Reflected boundary conditions are applied to the outer hex can.

2. The 91-pin single assembly configuration is based on the AFR-100 inner fuel assembly geometry (with and without wire wrap) including assembly duct and inter-assembly sodium gap. Reflected boundary conditions are applied to the outer hex can. The 91-pin geometry includes duct wall effects present in the actual AFR-100 assembly, although the effect of neighboring assemblies in the core is not accounted for due to the reflected geometry conditions.

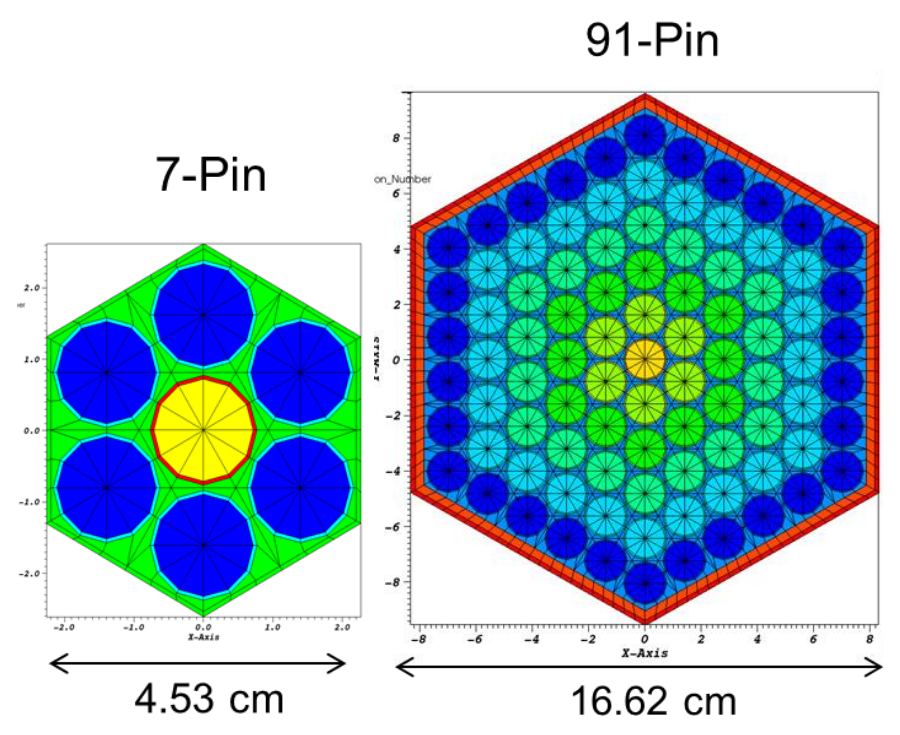

Figure 4.2.4 7-Pin (left) and 91-pin (right) configurations

The required high-fidelity calculations for the HCF evaluations are summarized in Table 4.2.1. The PROTEUS and Nek5000 references in this table indicates the required high-fidelity calculations by PROTEUS and Nek5000 codes.. 
Table 4.2.1. Required high-fidelity calculations for HCF evaluations

\begin{tabular}{|c|c|c|c|c|}
\hline \multirow{2}{*}{ Hot channel factors } & \multicolumn{2}{|c|}{ 7-pin } & \multicolumn{2}{|c|}{ 91-pin } \\
\hline & Bare bundle & With wire-wrap & Bare bundle & With wire-wrap \\
\hline \multicolumn{5}{|l|}{ Nominal Condition } \\
\hline & $\begin{array}{l}\text { PROTEUS } \\
\text { Nek5000 }\end{array}$ & Nek5000 & $\begin{array}{l}\text { PROTEUS } \\
\text { Nek5000 }\end{array}$ & Nek5000 \\
\hline \multicolumn{5}{|l|}{ Perturbed Conditions } \\
\hline Wire-wrap Orientation & & Nek5000 & & \\
\hline Cladding Thickness & & & $\begin{array}{l}\text { PROTEUS } \\
\text { Nek5000 }\end{array}$ & \\
\hline $\begin{array}{l}\text { Cladding Circumferential } \\
\text { Temperature Variation }\end{array}$ & & & Nek5000 & Nek5000 \\
\hline Cladding Properties & Nek5000 & & Nek5000 & \\
\hline Coolant Properties & Nek5000 & Nek5000 & Nek5000 & Nek5000 \\
\hline Fissile Mal-distribution & & & $\begin{array}{l}\text { PROTEUS } \\
\text { Nek5000 }\end{array}$ & \\
\hline Fuel Thermal Conductivity & & & Nek5000 & \\
\hline
\end{tabular}

Table 4.2.1 shows that PROTEUS calculations are needed in several spots, while Nek5000 calculations are needed at every spot. Generally, the PROTEUS calculations are not needed in most perturbed conditions because the impacts of the perturbed conditions on cladding, coolant, and wire-wrap orientation, etc. on neutronics characteristics (in particular, power distribution) are negligible. Thus, the power distributions at nominal condition without wire-wrap are sufficient to simulate the thermal-hydraulic perturbations. However, since the fissile maldistribution from manufacturing tolerance affects the neutronics characteristics, PROTEUS calculations are needed to evaluate the fissile maldistribution HCF. In this case, a stochastic analysis based on random sampling technique is used in this study because the distribution of the fissile content is statistically distributed in the assembly. Nek5000 calculations are needed in every HCF calculation because the change of thermo-physical properties may impact the peak temperatures of cladding, coolant, and fuel. In Table 4.2.1, the calculations in most HCFs are focused on the 91-pin bare bundle configurations by assuming that the wire-wrap effect on those HCFs are small. The comparison of the bare bundle and wire-wrap bundle are discussed in Appendix C.

\subsection{Neutronics Results for Hot Channel Factor Calculations}

For HCF calculations, iterations (i.e., data transfer and solution updates) between PROTEUS neutronics calculations and Nek5000 thermal-hydraulics calculations are needed at the nominal condition and at the perturbed state associated with uncertainties. The Nek5000 code needs power distributions, and the PROTEUS code needs temperature distributions. The peak temperature values obtained from the Nek5000 calculations are sensitive to the neutronics results, while the neutronics results are much less sensitive to the temperature variations (see the sensitivity studies in Appendix B). Thus, only one-way data transfer from PROTEUS to Nek5000 was considered, 
and the neutronics results at both nominal condition and perturbed status are discussed in this subsection. As discussed in Appendix B, the PROTEUS-MOCEX code was used for the neutronics analysis along with an offline coupling scheme to Nek5000.

The HCF calculations are performed for a representative 91-pin inner fuel assembly of the AFR100 core. This assembly is not coupled to other assemblies in the AFR-100 core, rather, the reflective boundary conditions imply it is part of an infinite lattice of inner fuel assemblies. The power level of the assembly was set at the average AFR-100 assembly power, $1.66667 \mathrm{MW}$, which is computed by the total power (250 MWth) divided by the number of fuel assemblies (150).

\subsubsection{Nominal Neutronics Results}

Detailed convergence studies were performed to determine the sensitivity of the neutronics result on mesh, angle, and energy discretization. The details are reported in Appendix B. Afterwards, axial power profiles for the nominal conditions of the 7-pin and 91-pin configurations were obtained with the discretization parameters listed in Section B.4.5 of Appendix B at a uniform $300 \mathrm{~K}$ temperature. To facilitate the data transfer to Nek5000, axial power profiles are normalized such that the power per unit length generated in the active core region has an average value of 1.0. The cost of the each simulation using the PROTEUS-MOCEX code is 8 core-hours (7-pin) and 61 core-hours (91-pin) on LCRC's Blues cluster.

The power profile for the 7-pin configuration is plotted in Figure 4.3.1.1. The peak/average power occurs at the top of the core with magnitude 1.9364, meaning that the power in this zone is $193 \%$ of the average axial power.

The power profile for the 91-pin configuration is plotted by ring in Figure 4.3.1.2. The peak/average power occurs at the top of the core with magnitude 1.7654 in the center pin, meaning that the power in this zone is $176 \%$ of the average axial power. The pins in other rings have nearly identical peak/average values, the largest deviation being pins in ring 6 which have $0.88 \%$ higher average/peak than the center pin. The average pin powers are reported in Table 4.3.1.1, which shows that both the pin power magnitudes and axial shapes are nearly identical in each ring. 


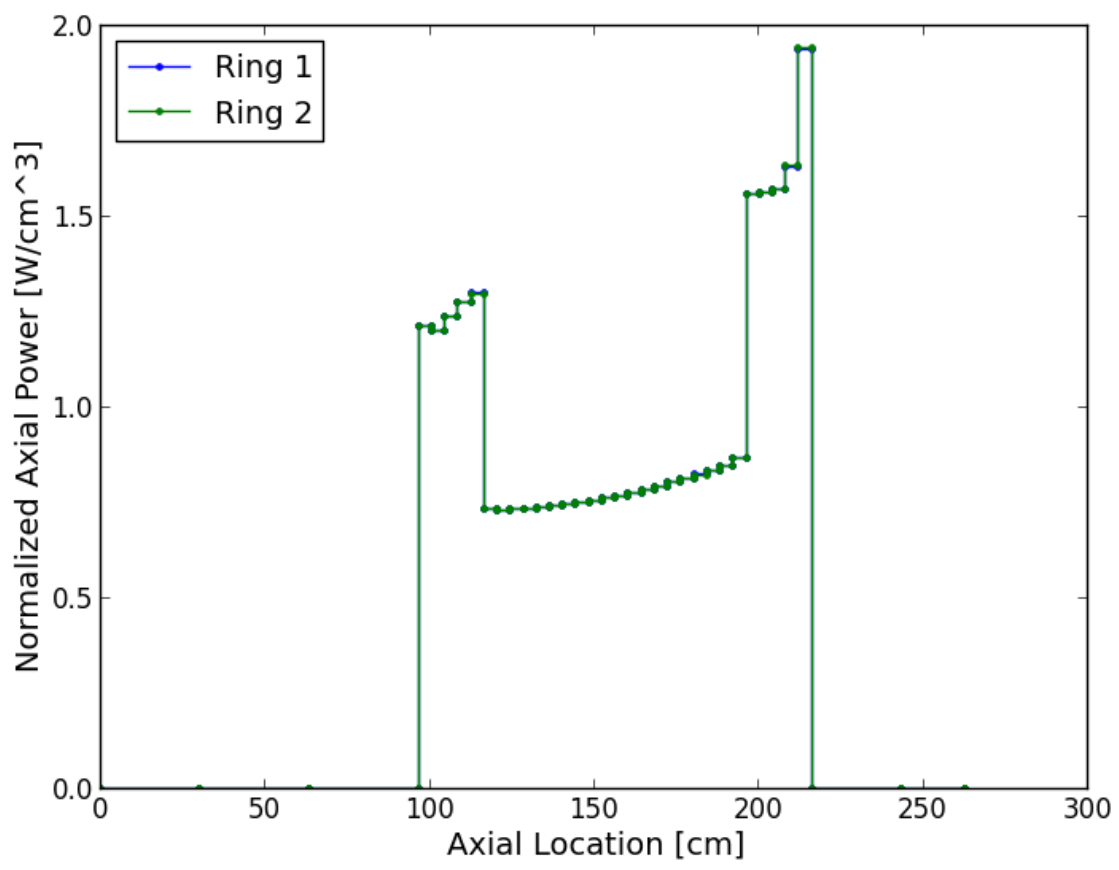

Figure 4.3.1.1. MOCEX nominal condition axial power profile (7-pin)

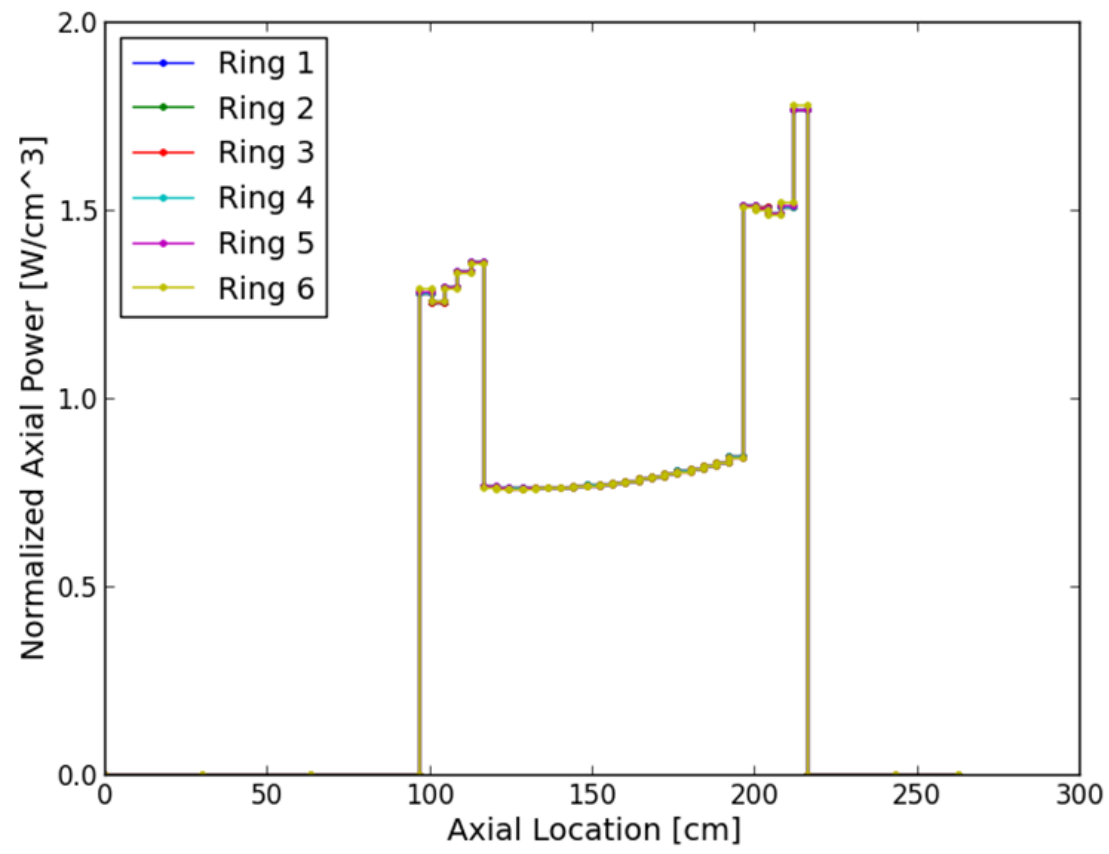

Figure 4.3.1.2. MOCEX nominal condition axial power profile (91-pin) 
Table 4.3.1.1. MOCEX nominal condition average pin power in each ring (91-pin)

\begin{tabular}{|l|l|l|}
\hline Ring & $\begin{array}{l}\text { Average } \\
\text { Power per Pin } \\
(\mathrm{kW})\end{array}$ & $\begin{array}{l}\text { Peak/Average } \\
\text { Axial Power } \\
\text { per Pin }\end{array}$ \\
\hline 1 (center) & 18.319 & 1.7654 \\
\hline 2 & 18.310 & 1.7651 \\
\hline 3 & 18.300 & 1.7649 \\
\hline 4 & 18.302 & 1.7652 \\
\hline 5 & 18.321 & 1.7673 \\
\hline 6 (next to duct) & 18.324 & 1.7810 \\
\hline
\end{tabular}

As previously noted, the 7-pin and 91-pin axial power profiles do not follow a cosine shape along the axial dimension due to the unique enrichment pattern in the assembly, where the top and bottom regions are highly enriched (18.0\%) compared to the middle region (8.8\%). However, even more notably, the top-most region of the active fuel zone is the highest power zone in the pin. This power peaking happens to occur in the zone where the sodium coolant is also highest temperature and is likely to significantly impact the hot channel factor calculations. The peak/average axial power for the 7-pin calculation is 1.9364 and that of the 91-pin calculation is 1.7654 . Therefore, the presence of the duct and inter-assembly gap decrease the overall power peaking along the axial dimension in the 91-pin configuration.

To further confirm and understand this behavior, a conventional 33-group DIF3D neutronics simulation using homogenized nodes on each axial plane was performed to reproduce the general behavior seen here (power peaking just below gas plenum). The detailed axial power distribution was reconstructed using a post-processing tool. The DIF3D simulation confirmed the general axial power shape, although the power peaking in the upper fuel zone (essentially just the top value in the figure which represents the power vs the axial average power) was calculated to be far less severe (1.6582 vs. 1.9364). The DIF3D simulation calculated the eigenvalue to be 1.12212 vs the "converged" MOCEX value of 1.12708 using the same 33g cross section sets. The differences are due to the high fidelity simulation capturing much more details effects particularly in the regions where the material changes. We note that a full detailed space-angle-mesh study was not performed by DIF3D here, but that DIF3D does have a limit on how finely detailed the axial mesh can be. 


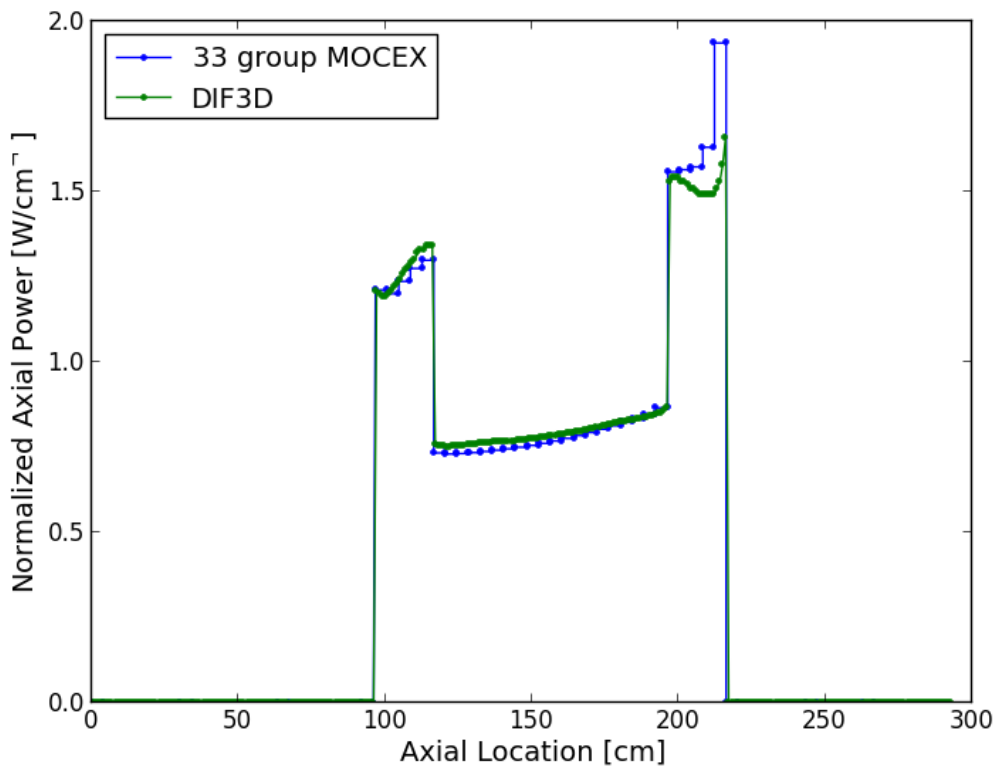

Figure 4.3.1.3 Axial power profile comparison between DIF3D and MOCEX (7-pin)

Once the power peaking was confirmed by conventional tools, the individual multigroup fluxes were examined to understand the origin of this behavior, which was somewhat unexpected and will impact the peak coolant temperature and subsequently the hot channel factors being calculated.

The power profile as well as multigroup fluxes from group 1 [10-14.190675 MeV], group 8 [301.97-497.87 keV], and group 25 [61.4-101.3 eV] are plotted in Figure 4.3.1.4 for the 33-group 7-pin calculation. Only the active core zones are shown for better visibility. The dramatic power peaking at the top of the fuel pins can be observed on the far left figure. The fast fluxes (groups 1 and 8) are not peaked in that axial zone, so fast fission is not the sole contributor of increased power in that zone.

However, peaks are seen in the group 25 flux (and subsequent thermal groups) as neutrons slow down and reflect back into the core from the sodium bond-filled gas plenum located directly above the active fuel. Since the fission cross section for U-235 is 1-3 orders of magnitude higher at epithermal and thermal energies as opposed to the fast energy groups (see Figure 4.3.1.5), the thermal fission is substantially increased in this zone. In fact, while the lower energy group flux in the power-peaked zone is two orders of magnitude lower than the fast flux $\left(10^{10} \mathrm{vs} 10^{12}\right)$, thermal fission contributes nearly as much energy in this zone as fast fission, leading to significantly higher total power production.

In conclusion, the nominal condition 7-pin and 91-pin configurations were generated successfully using the PROTEUS-MOCEX code. The center pin axial power distribution and magnitude accurately describes all of the pins, and thus is the only data needed for Nek5000. 


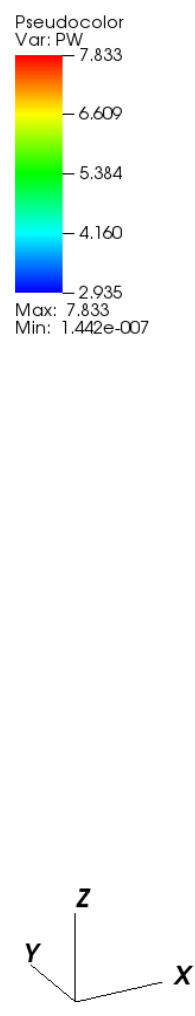

(a)
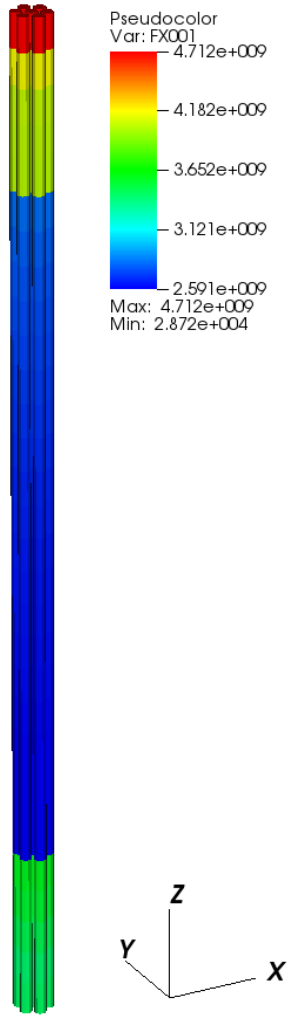

(b)
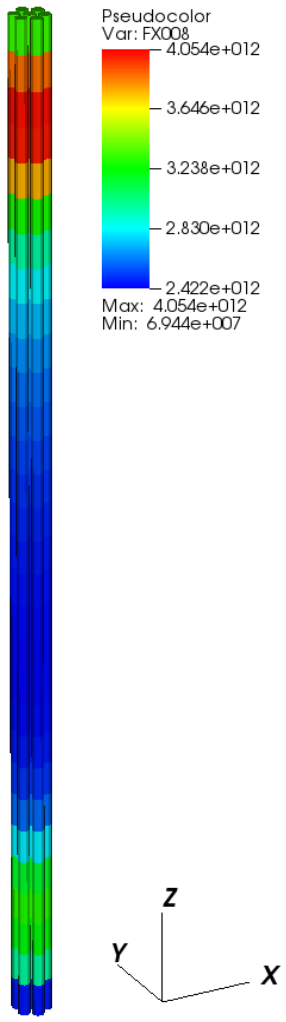

(c)

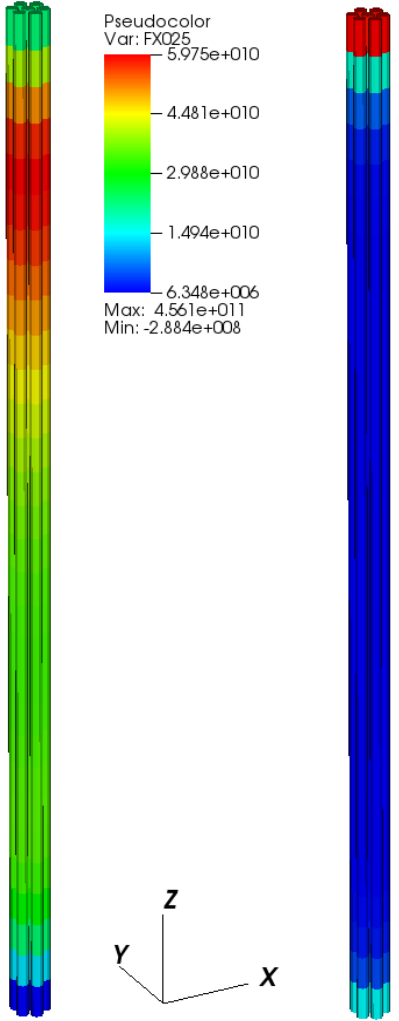

(d)

Figure 4.3.1.4 Axial profiles for computed by 33g MOCEX simulation (7-pin)

((a) axial power profile in active core (b) group 1 flux, (c) group 8 flux, and (d) group 25 flux)

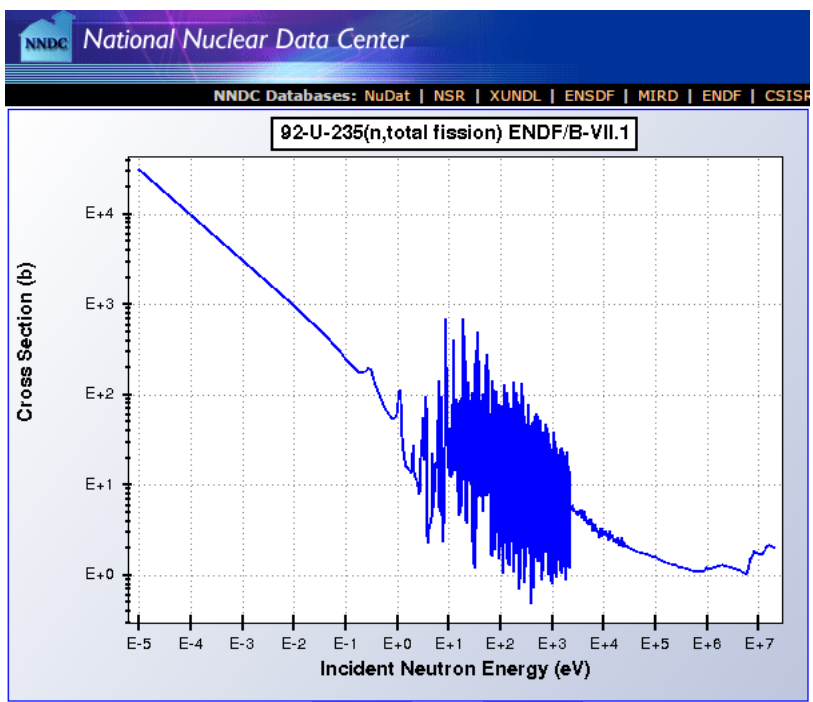

Figure 4.3.1.5 Fission cross section for $\mathrm{U}-235$ retrieved

(from National Nuclear Data Center website) 


\subsubsection{Neutronics Results Associated with Uncertainties in Thermo-Physical Properties}

The HCF associated with the uncertainties in thermo-physical properties quantifies the effect of uncertainties in coolant density, cladding thermal conductivity, fuel thermal conductivity, etc. These properties are related solely to the thermal-hydraulics calculation, with the exception of coolant density, which may have a neutronics effect but is unlikely to change the normalized axial power profile if changed uniformly and in modest amounts. Therefore, the nominal condition power profile already calculated was provided to Nek5000 for these perturbation calculations.

\subsubsection{Neutronics Results Associated with Uncertainties in Subchannel Flow Area}

The focus of this HCF is to quantify the effect of uncertainties in subchannel flow area (area between pins) due to manufacturing tolerances in the fuel pin cladding. The manufacturing tolerance on the cladding thickness was obtained from the EBR-II MARK-V fuel fabrication, which is $+/-3 \%$ [Ku 1994]. In addition, based on the communications with the fuel experts who are currently involved in the SFR metallic fuel development, the manufacturing tolerance on the cladding thickness of $+/-0.05 \mathrm{~mm}$ was also considered [Cheon 2017]. The cladding thickness of the center pin only was reduced for this study. With more compute time available and more time for meshing, the fuel pin cladding radius should be varied randomly according to a distribution. The presence of wire wrap with asymmetric fuel pin geometries causes severe meshing implications which are time consuming to resolve and to study.

Reducing the cold cladding thickness yields the new cladding dimensions in Table 4.3.3.1. The $0.05 \mathrm{~mm}$ reduction in thickness is a more dramatic scenario than reducing by $3 \%$ and results in a new cladding thickness of $0.473 \mathrm{~mm}$ (vs $0.508 \mathrm{~mm}$ ). This small geometry perturbation is depicted in Figure 4.3.3.1 and is the only one considered for neutronics. As seen in the following tables, the effect on the power profile is negligible and the effect one eigenvalue is very small. Using the MARK-V perturbation would result in an even smaller effect.

Table 4.3.3.1 Cold cladding thickness perturbations and corresponding hot dimensions

\begin{tabular}{|l|l|l|l|l|l|}
\hline & $\begin{array}{l}\text { Cold Clad } \\
\text { Thickness } \\
(\mathbf{c m})\end{array}$ & $\begin{array}{l}\text { Hot Inner } \\
\text { Clad } \\
\text { Radius } \\
(\mathbf{c m})\end{array}$ & $\begin{array}{l}\text { Hot Outer Clad } \\
\text { Radius (Bare } \\
\text { Bundle) }(\mathbf{c m})\end{array}$ & $\begin{array}{l}\text { Hot Outer Clad } \\
\text { Radius (w/ } \\
\text { Smeared WW) } \\
(\mathbf{c m})\end{array}$ & $\begin{array}{l}\text { Hot Clad Thickness } \\
\mathbf{( w / ~ S m e a r e d ~ W W ) ~} \\
(\mathbf{c m})\end{array}$ \\
\hline Unperturbed & 0.0500 & 0.6993 & 0.7496 & 0.7516 & 0.0523 \\
\hline $\begin{array}{l}\text { Thickness } \\
\text { reduced by 3\% }\end{array}$ & 0.0485 & 0.6993 & 0.7481 & 0.7501 & 0.0508 \\
\hline $\begin{array}{l}\text { Thickness } \\
\text { reduced by 0.05 } \\
\text { mm }\end{array}$ & 0.0450 & 0.6993 & 0.7446 & 0.7466 & 0.0473 \\
\hline
\end{tabular}




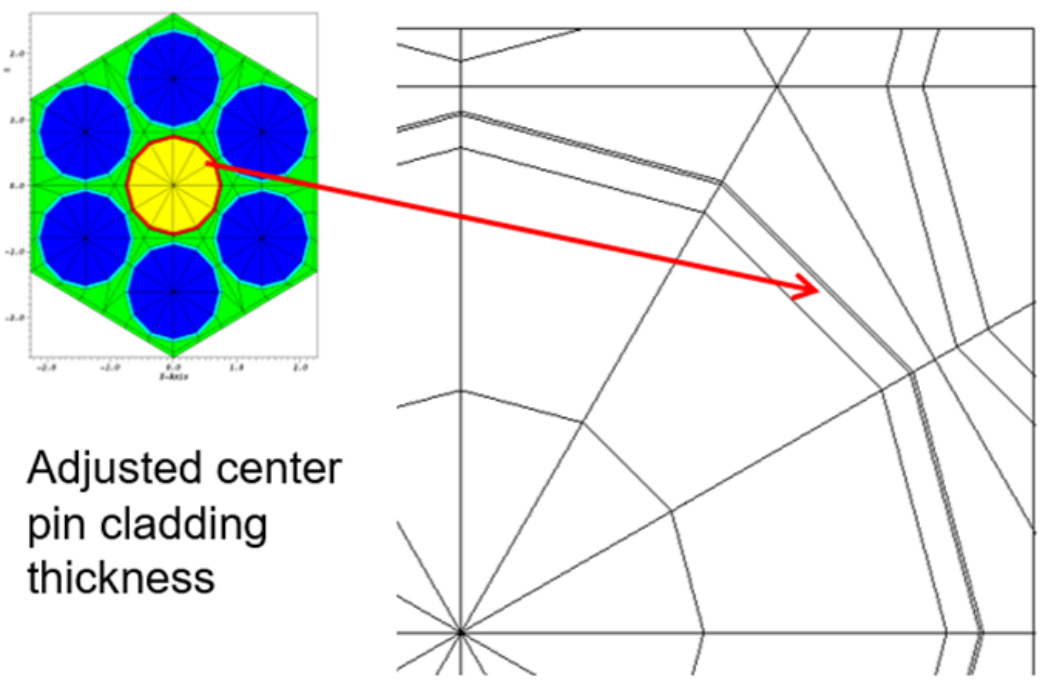

Figure 4.3.3.1 Overlay of the initial and perturbed meshes to show cladding perturbation ($0.05 \mathrm{~mm}$ change in cladding thickness).

Table 4.3.3.2 MOCEX eigenvalue results for cladding perturbation (7-pin)

\begin{tabular}{|l|l|l|l|l|l|l|l|l|}
\hline Case & $\begin{array}{l}\text { Energy } \\
\text { Group }\end{array}$ & Eigenvalue & $\begin{array}{l}\text { \% of Total } \\
\text { Power in } \\
\text { Center Pin }\end{array}$ & Temp. & $\begin{array}{l}\text { Angular } \\
\text { Cubature }\end{array}$ & $\begin{array}{l}\text { Mesh } \\
\text { Elements } \\
(2 \mathrm{D})\end{array}$ & $\begin{array}{l}\text { Mesh } \\
\text { Refinement }\end{array}$ & Planes \\
\hline Unperturbed & 33 & 1.12708 & $16.657 \%$ & $300 \mathrm{~K}$ & L3T5 & 265 & A1R1 & 78 \\
\hline $\begin{array}{l}\text { Clad } \\
\text { thickness } \\
\text { reduced by } \\
0.05 \mathrm{~mm}\end{array}$ & 33 & 1.12742 & $16.667 \%$ & $300 \mathrm{~K}$ & L3T5 & 265 & A1R1 & 78 \\
\hline
\end{tabular}

Table 4.3.3.3 MOCEX eigenvalue results for cladding perturbation (91-pin)

\begin{tabular}{|l|l|l|l|l|l|l|l|l|}
\hline Case & $\begin{array}{l}\text { Energy } \\
\text { Group }\end{array}$ & Eigenvalue & $\begin{array}{l}\% \text { of Total } \\
\text { Power in } \\
\text { Center Pin }\end{array}$ & Temp. & $\begin{array}{l}\text { Angular } \\
\text { Cubature }\end{array}$ & $\begin{array}{l}\text { Mesh } \\
\text { Elements } \\
\text { (2D) }\end{array}$ & $\begin{array}{l}\text { Mesh Refinement } \\
\text { Rlanes }\end{array}$ & A1R1 \\
\hline Unperturbed & 33 & 1.10327 & $1.09856 \%$ & $300 \mathrm{~K}$ & L3T5 & 3061 & 78 \\
\hline $\begin{array}{l}\text { Clad } \\
\text { thickness } \\
\text { reduced by } \\
0.05 \mathrm{~mm}\end{array}$ & 33 & 1.10330 & $1.09864 \%$ & $300 \mathrm{~K}$ & L3T5 & 3061 & A1R1 & 78 \\
\hline
\end{tabular}

The 7-pin configuration displayed a $+34 \mathrm{pcm}$ change in eigenvalue due to the $0.05 \mathrm{~mm}$ decrease in cladding thickness, but a negligible change in axial power profile or in the power distribution among pins. The 91-pin configuration displayed only a $+3 \mathrm{pcm}$ change in eigenvalue compared to the unperturbed case, and the axial power profile changed negligibly. This is reasonable because the 91-pin configuration has 90 unperturbed pins and only 1 perturbed pin (only 1\% of pins are 
perturbed) whereas the 7-pin configuration has 6 unperturbed pins and 1 perturbed pin (14\% of pins are perturbed). The 7-pin and 91-pin axial power profiles are displayed in Figure 4.3.3.2 and Figure 4.3.3.3. The percent of total power in the center pin is reported in the previous tables.

In conclusion, the effect of the cladding perturbation in neutronics is negligible. The neutronics calculations were not repeated for the MARK-V cladding perturbation (3\% reduction in cladding thickness) because the perturbation is even smaller. Using the unperturbed (nominal) condition power profiles in Nek5000 for the cladding perturbation hot channel factor calculation is therefore justified.

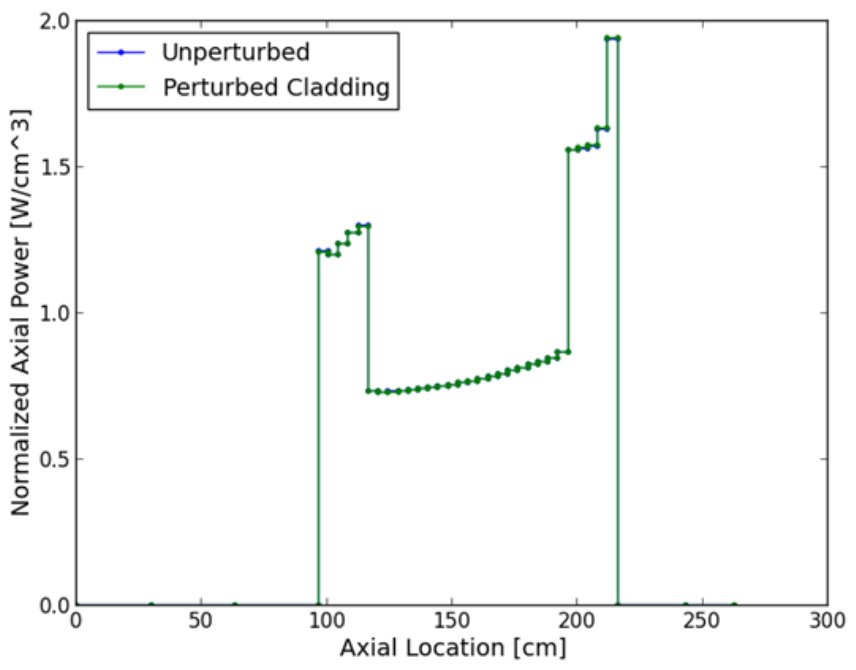

Figure 4.3.3.2 MOCEX axial power profile for cladding thickness change (7-pin)

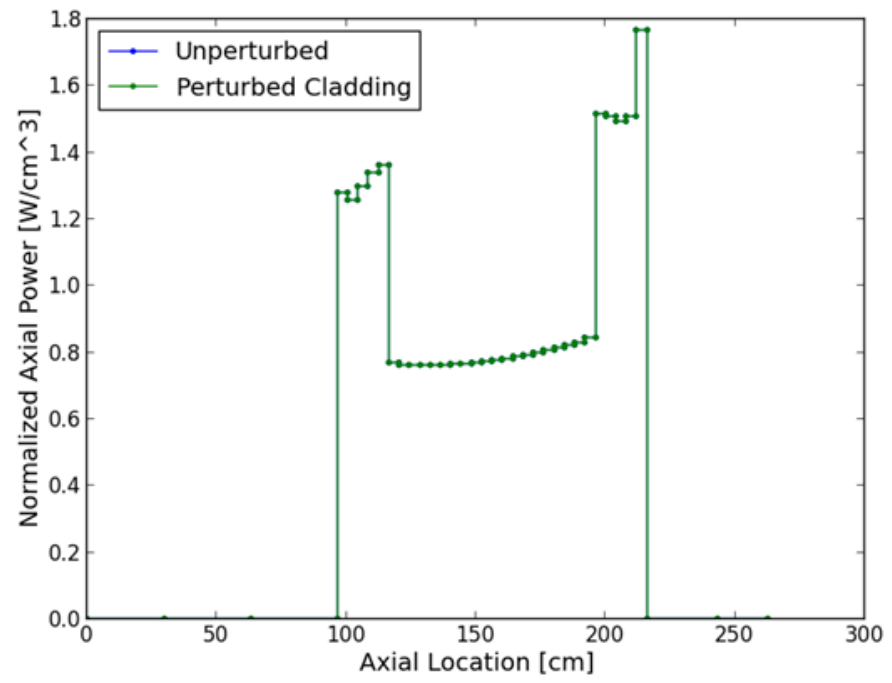

Figure 4.3.3.3 MOCEX axial power profile for cladding thickness change (91-pin) 


\subsubsection{Neutronics Results Associated with Uncertainties in Fuel Pellet Fissile Content}

Due to manufacturing uncertainty, the U-235 fissile content in a pin can vary up to $+/-6 \%$ from the target enrichment. This variability in enrichment causes changes in local pin power and temperature. Since it is difficult to know the variation of enrichment of each pin, a stochastic (random sampling) technique was used to assess the manufacturing fissile content uncertainty. To obtain meaningful stochastic distributions, thirty independent neutronics inputs for the 91-pin configuration were randomly selected as follows:

1. The fissile content in each pin is sampled individually and not affected by neighboring pins.

2. The bottom and top enrichment zones of a pin are assumed to have identical enrichments since the bottom and top fuel pellets have identical target enrichments and may be loaded from the same batch of fuel. This is a conservative assumption because it forces the top and bottom zones to have high enrichments simultaneously if a high enrichment is sampled, and thus the pin will have maximum integrated axial power from the nominal case.

3. A normal distribution is used to sample each enrichment perturbation, with mean $=0 \%$ and 1 -sigma $=2 \%(3$-sigma $=6 \%)$. In such a distribution, more than $99 \%$ of samples will lie within $6 \%$ of the mean, meaning that more than $99 \%$ of fuel pins will have enrichments within $6 \%$ of the target manufacturing value.

4. The sampled enrichment perturbations are rounded to the nearest integer in the discrete set $\{ \pm 6, \pm 5, \pm 4, \pm 3, \pm 2, \pm 1,0\} \%$. Limiting the enrichment perturbations to integers simplifies the definition of material compositions in neutronics.

The eigenvalue distribution and peak pin power from each of the thirty simulations was identified in an attempt to characterize the corresponding distributions as shown in Figure 4.3.4.1 and Figure 4.3.4.2. The eigenvalue histogram uses bin widths of $25 \mathrm{pcm}(0.00025)$, and the eigenvalue resembles a normal distribution. The bin widths in the peak pin power histogram are $0.05 \mathrm{~kW}$ (50 Watts). However, the distribution of peak pin powers does not follow a defined distribution, most likely due to the low number of samples. In future work, we recommend that much more than thirty simulations be performed because the axial heterogeneity of the fuel pin allows for more variability than a single enrichment zone pin. The peak pin power should follow a more predictable distribution when provided with more samples.

Due to the relatively small number of samples (30), the simulation with the maximum peak pin power was conservatively chosen to transfer power to Nek5000. A normal distribution would be more likely to occur with more random sampling and the simulation with a peak pin power lying in the $95 \%$ confidence interval (2-sigma) could be selected as in Figure 4.3.4.3. 


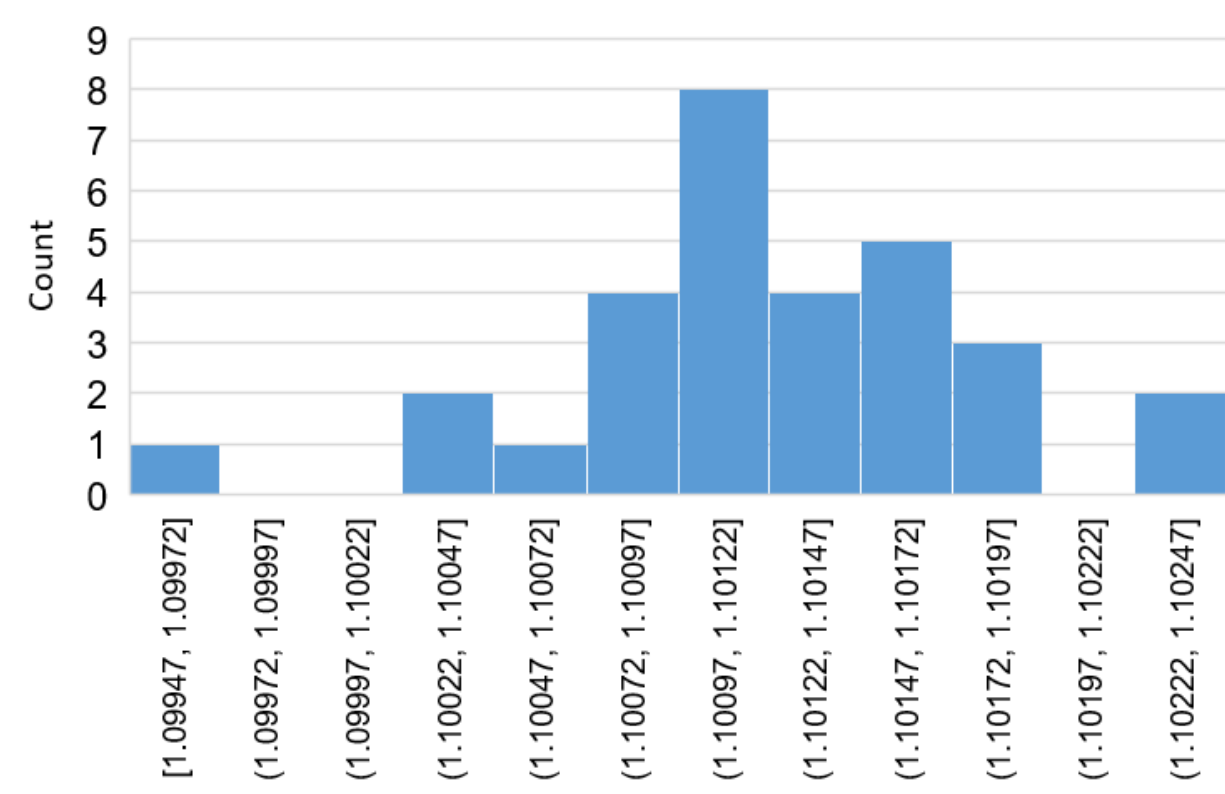

Eigenvalue

Figure 4.3.4.1. Eigenvalue distribution based on 30 random samples.

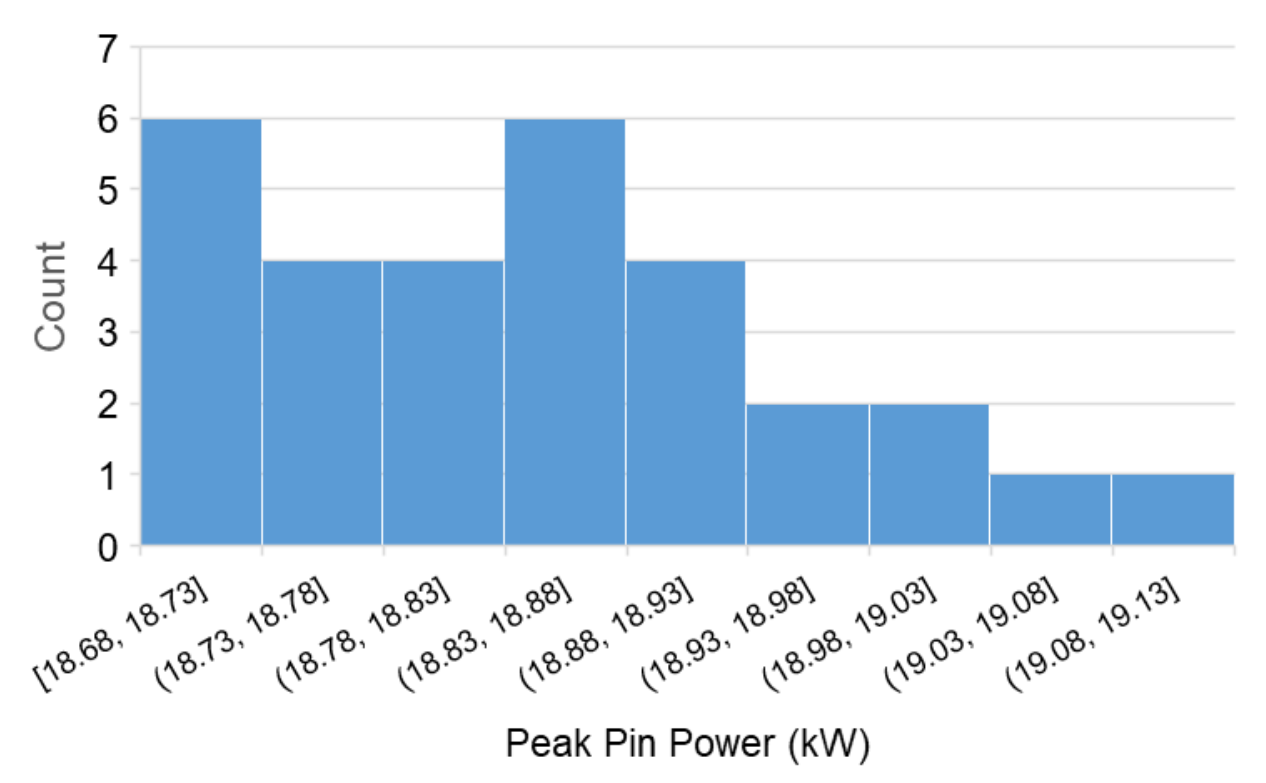

Figure 4.3.4.2. Peak pin power distribution based on 30 random samples. 


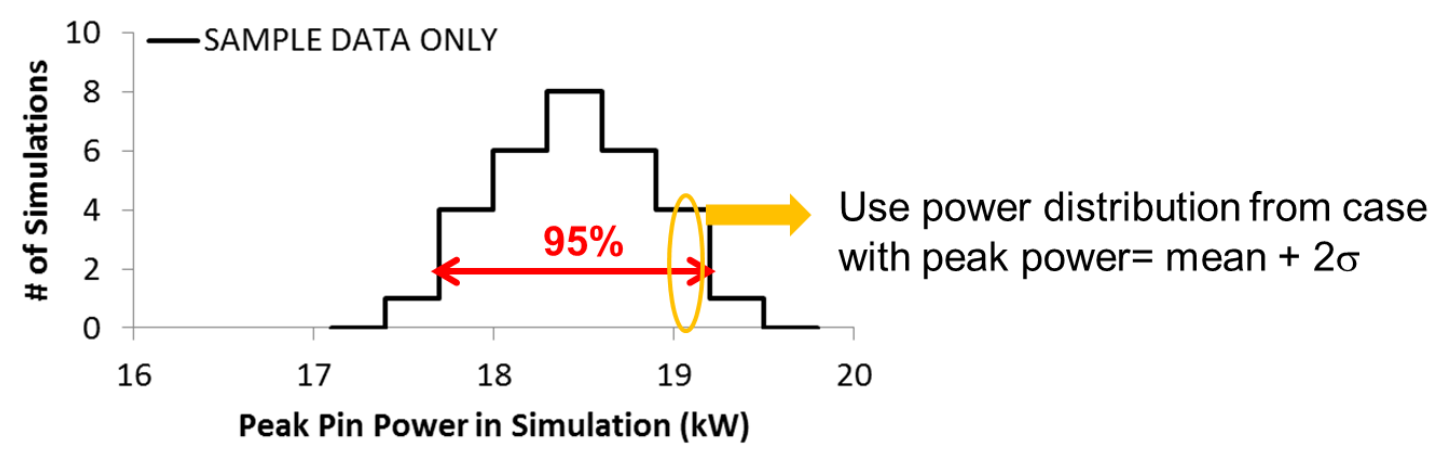

Figure 4.3.4.3. Hypothetical peak pin power distribution based on random sampling with a very large number of samples.

The peak pin power occurring out of all thirty simulations was $19.082 \mathrm{~kW}$ (pin 41 in ring 5 of Simulation 25). This particular pin was randomly assigned $+7 \%$ enrichment perturbation to the top/bottom zones, and $+4 \%$ perturbation to the middle zone. The $+7 \%$ value was outside of the $99 \%$ confidence interval (recall that $99 \%$ of samples are likely to be within $6 \%$ of the target enrichment) but this is not unexpected because out of 5,460 enrichment samples (91 pins/simulation*2 zones/pin*30 simulations), roughly 15 samples are expected to lie outside this interval. However, this large perturbation did not occur in the center fuel pin where powers are naturally highest due to the intra-assembly flux distribution, therefore the effect is somewhat mitigated. The distribution of enrichment perturbations from Simulation 25 are plotted in Figure 4.3.4.4 and are shown to follow a normal distribution, as specified. The yellow line represents a normal distribution with the mean 0 and standard deviation 2, and the blue bars represent the 91 samples within a single simulation. The data closely follows a normal distribution, with some deviation due to the limited number of samples (91 top/bottom zone samples, and 91 middle zone samples). 

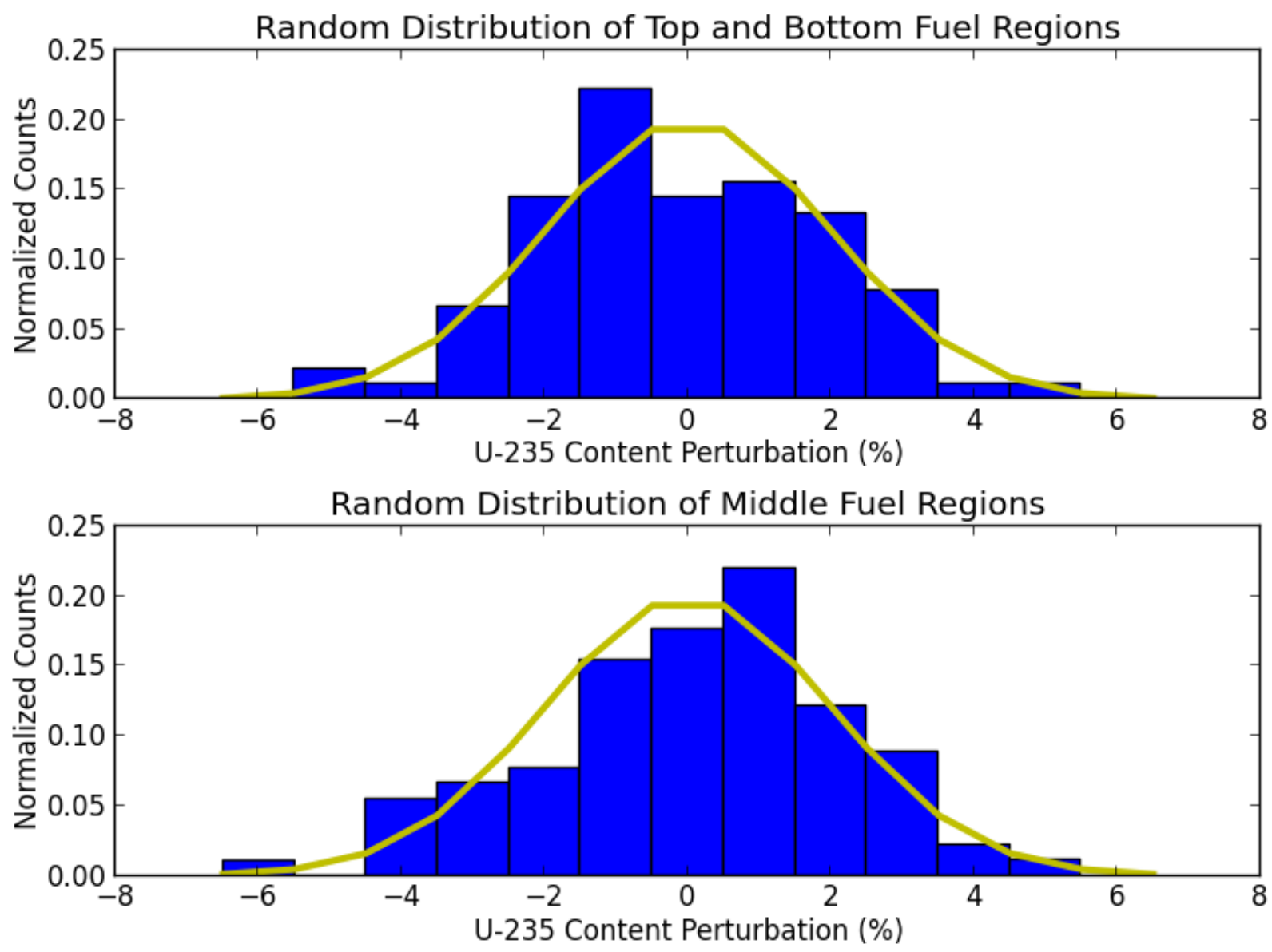

Figure 4.3.4.4. Normal distribution of enrichment variation for Simulation 25. 


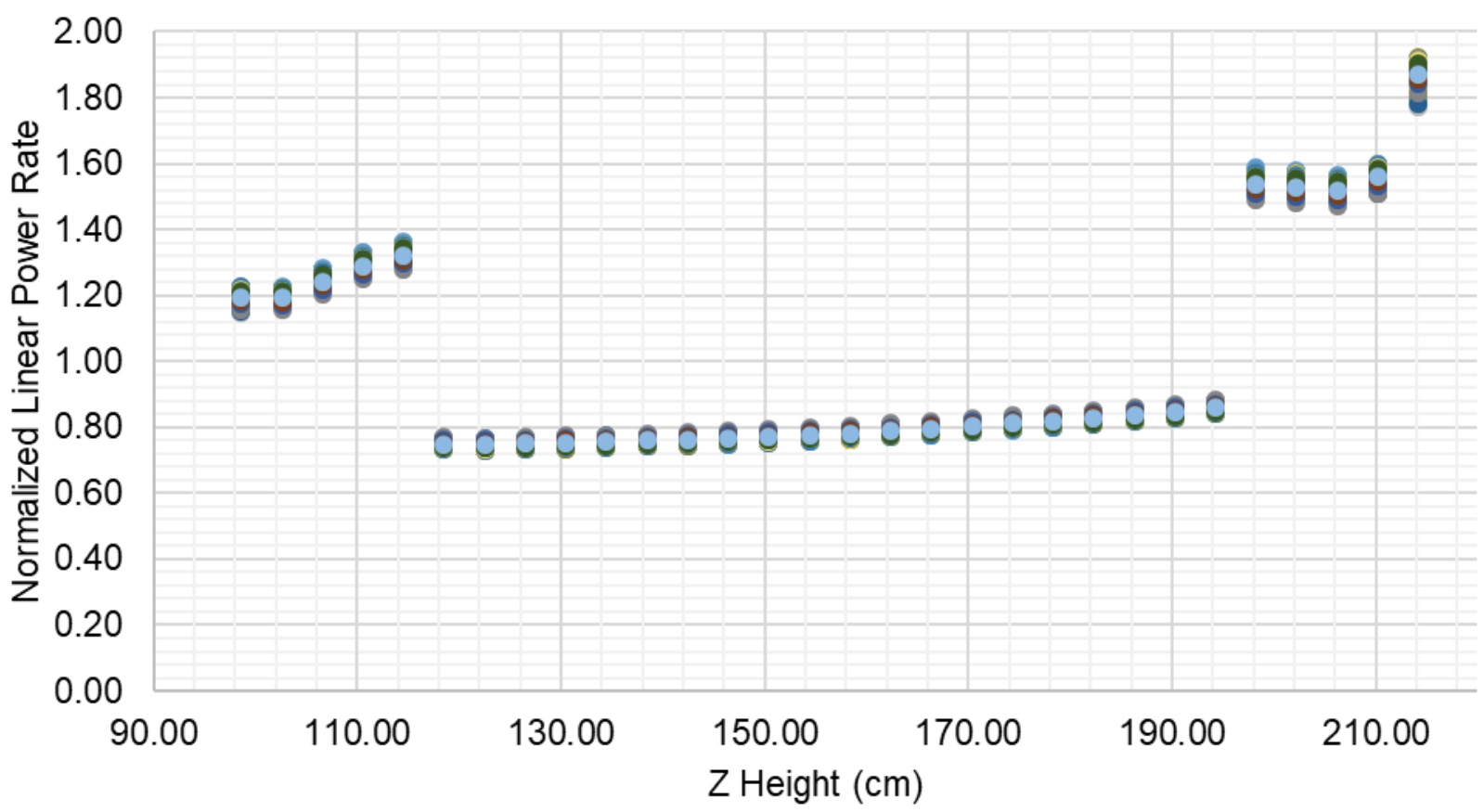

Figure 4.3.4.5. Normalized axial power distribution for all 91 pins within Simulation 25.

The normalized axial power distribution for each pin needed for Nek5000 is plotted in Figure 4.3.4.5. The figure demonstrates that the axial power shape is not significantly impacted by the local enrichment perturbations. Therefore, the same axial power shape can be used for all of the pins in Nek5000, where the scaling factor (pin power) is unique.

Figure 4.3.4.6 depicts the pin power distribution at the active core outlet $(\mathrm{z}=216 \mathrm{~cm})$ for the nominal case (left) and the perturbed condition bounding case (right). The nominal case assumes all pins are manufactured with the targeted fissile content. The perturbed case shown is from simulation 25, the bounding case used for Nek5000 calculations, where the pins exhibit randomized fissile content perturbations according to a normal distribution described in this section. The color scale is identical in the two figures although the values are normalized and not directly translatable to Watt units. The maximum pin power occurs in pin 41, ring 5 (seen as a red color in the figure). The maximum pin power in the perturbed case is $19.082 \mathrm{~kW}$, and the average pin power is 18.276 $\mathrm{kW}$. The total heat generation in the 91 fuel pins is $1.663070 \mathrm{MW}$, and the heat generation outside the fuel pins is $3.595 \mathrm{~kW}$, or $0.2 \%$ of the total assembly power $(1.666665 \mathrm{MW})$. The heat generation outside the fuel pins is ignored in Nek5000.

We note that the nominal neutronics calculation used the same total assembly power $(1.666665$ MW) as in the perturbed calculation, but the pin power values were post-processed to Nek5000 by smearing all power into the fuel pins to yield an average pin power of $18.315 \mathrm{~kW}$ (whereas in reality, some of this power should have been generated in the coolant and duct). Therefore, the nominal pin powers are about $0.2 \%$ higher than the average pin power in the perturbed calculation. 
Ideally, the nominal calculation should be repeated to remove this smeared normalization assumption, however, the bias of about $-40 \mathrm{~W}$ per pin is very small compared to the pin power variations induced by the local fissile content perturbations, which in the peak pin case is $+807 \mathrm{~W}$ above the average pin power. Therefore, this procedure is justifiable, but in the future the data should be processed in a more consistent manner, since the perturbation of interest may not be large in comparison to this calculation bias.
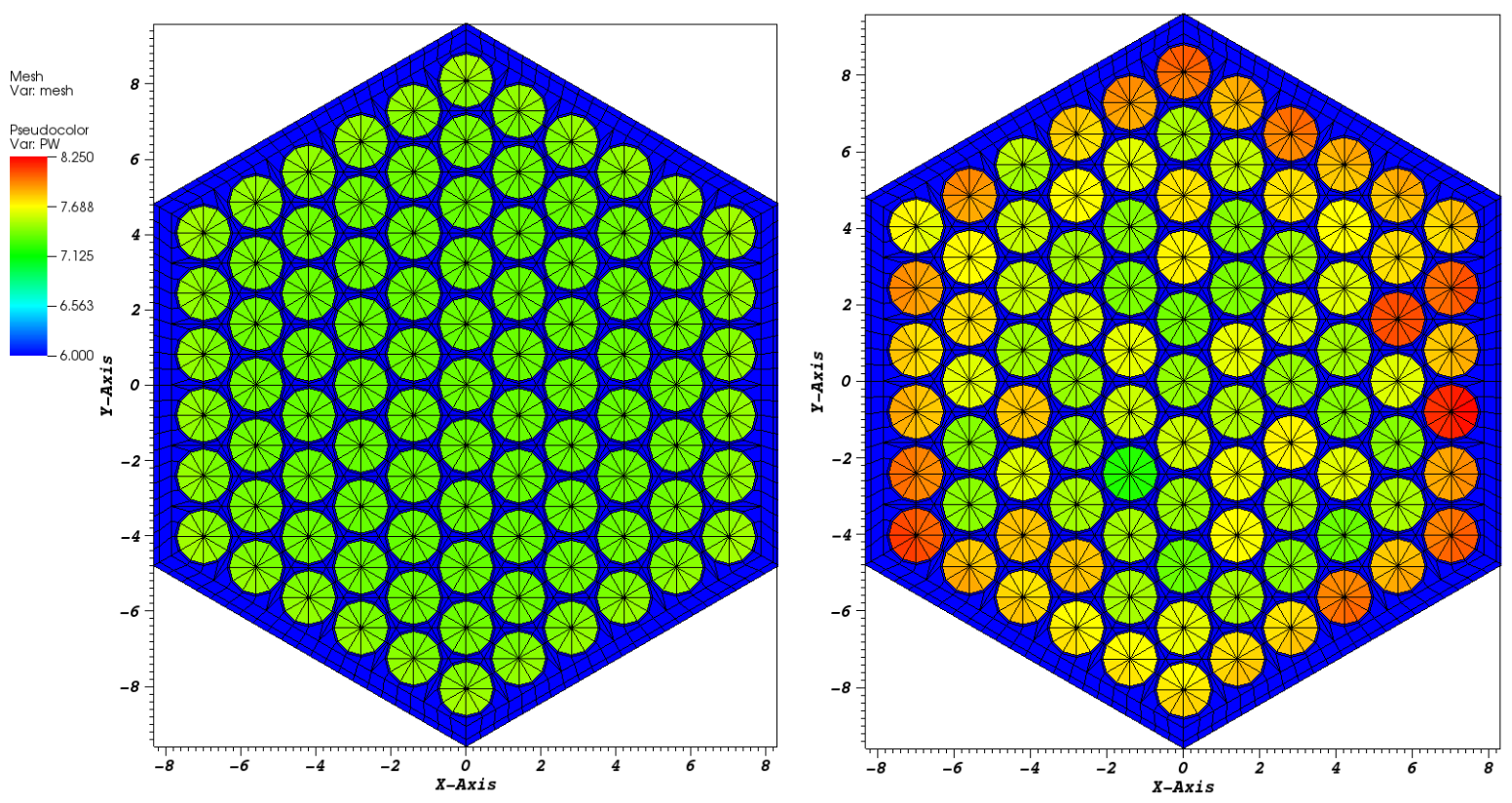

Figure 4.3.4.6. Power distribution at active core outlet from nominal condition (left) and randomized fissile content bounding case (right).

\subsection{Thermal-Hydraulic Results for Hot Channel Factor Calculations}

Nek5000 calculations are computationally expensive due to the inclusion of high-fidelity features. Significant manpower is also required to generate the input finite element meshes. So, before moving forward, detailed sensitivity studies were conducted to save computational resources and ensure the reliability of the calculations, which include the test of mesh generation schemes and performance comparison between bare rod bundle and rod bundle with wire-wrap with the commercial computational fluid dynamics code of STAR-CCM+. The full details are reported in Appendix C. Additionally, when constructing a simulation for flow through a wire-wrapped fuel rod bundle, scientists may utilize a smaller bundle than design in order to minimize material or computational costs. The 7-pin rod bundle is the smallest bundle that can reasonably replicate the full assembly geometry. However, small bundles may not capture the required flow physics in larger bundles [Brockmeyer 2017]. Therefore, simulations on the full-size rod bundle (91-pin) are also conducted for assessment against the 7-pin bundle. Hence, the hot channel factor calculations 
are performed for two geometry types: a 7-pin configuration based on AFR-100 with and without wire wrap and a 91-pin full AFR-100 fuel assembly with and without wire wrap.

\subsubsection{Bare Rod Bundle Results}

The pin pitch/diameter (P/D) ratio for AFR is about 1.08, which makes the assembly a tight lattice. The flow in tight rod bundles is characterized by long-term, large-scale coherent patterns in the stream-wise direction. Therefore, unsteady simulations are necessary if accuracy in the prediction of averaged statistics is needed. Since the Reynolds number in the AFR-100 assembly can reach 25,000, it is very expensive to perform the calculation with Large Eddy Simulation (LES)methodology. Some literatures ([Yu 2011], [Merzari 2008]) point out that Unsteady Reynolds-Averaged Navier-Stokes (URANS) simulation perform equally well, and solve for the transient solution at a fraction of the computational cost of large eddy simulation (LES). Therefore, URANS simulation with $\mathrm{k}-\omega$ turbulence model is applied for this study. URANS simulations as performed in Nek5000 are more accurate than RANS simulations as performed in STAR-CCM+ because they capture the coherent structure in a tight lattice, which is impossible to reproduce with RANS simulation.

Three axial power distributions are applied to the 7-pin bundle simulation: uniform power distribution, the core average BOC power distribution as estimated from initial design report [Grandy 2013], and the power distribution for the central fuel pin of the inner-core type fuel assembly calculated from PROTEUS. Uniform power distribution is the most common power distribution when performing CFD standalone calculations since neutronics information is either unnecessary or unavailable. The axial power distribution from PROTEUS is a representative axial power profile of the AFR-100 assembly which may have peak temperatures based on the heterogeneous fissile distribution as shown in Figure 3.1.2, rather than the core average BOC power. These three power distributions are shown in Figure 4.4.1.1. The power distribution from PROTEUS deviates greatly from the uniform and core-average BOC power distributions, as explained in Section 4.3.1. The PROTEUS calculation also indicates that the deviation of the radial power distribution (power distribution from pin to pin) is negligibly small. Thus, the power distribution is considered radially uniform in the simulation. 


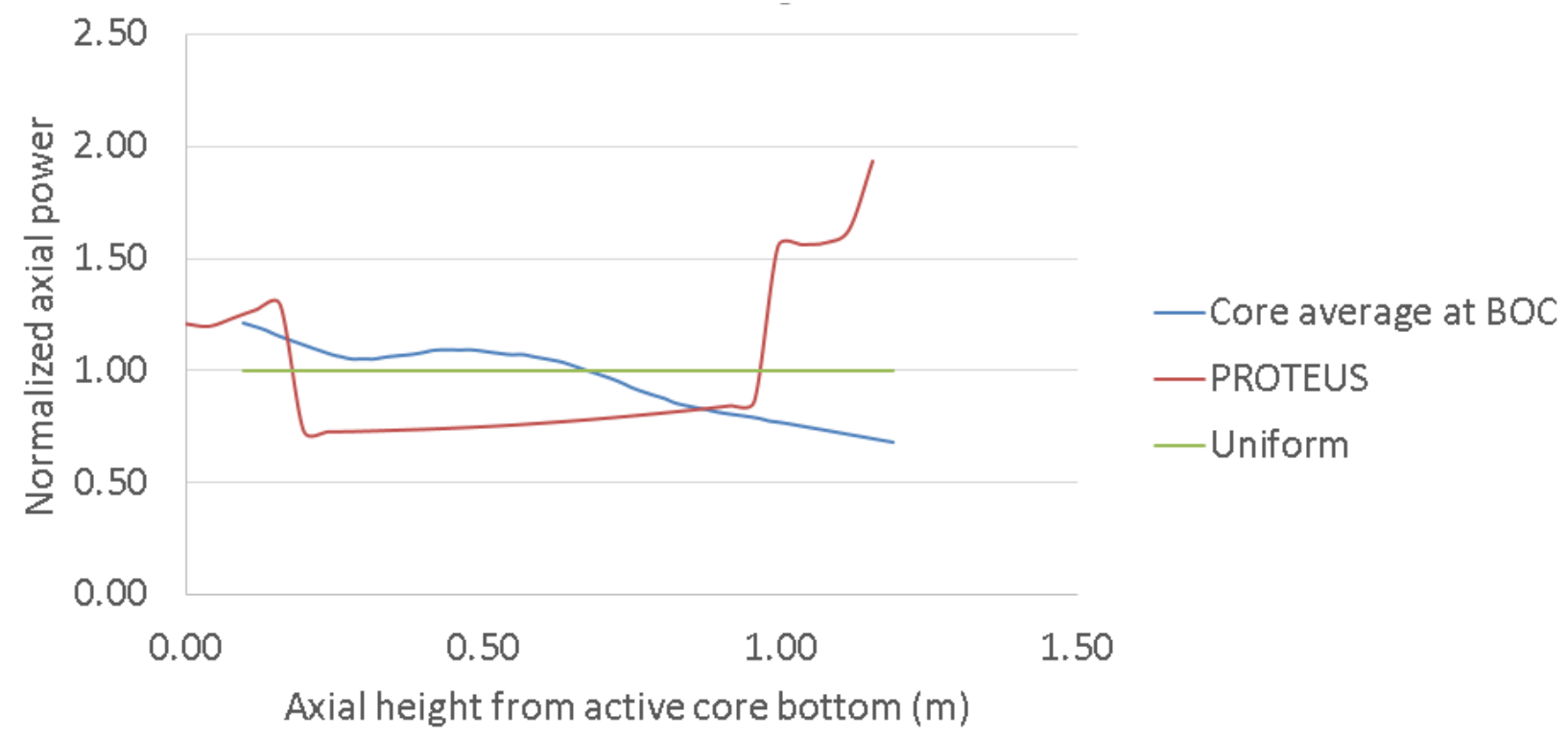

Figure 4.4.1.1 Three axial power distributions applied to 7-pin bare rod bundle

Figure 4.4.1.2 shows the temperature distributions at different elevations within the last wirewrap pitch (uppermost region of the active core) calculated using the power distribution from PROTEUS. The temperature is hotter in the center and cooler in the edge due to slightly increased amounts of coolant near the periphery of the 7-pin configuration. The magnitude of the temperature increases along the flow direction.

Figure 4.4.1.3 shows the outlet temperature with different axial power distribution. The average coolant temperature stays the same in the three cases despite the very different power distributions. Since the power rate reaches maximum at outlet in the PROTEUS distribution while it reaches minimum at outlet in the BOC distribution, the maximum fuel temperature difference at outlet between these two cases is large. The maximum fuel temperature using the power distribution from PROTEUS is $1010 \mathrm{~K}$ while it is $896.1 \mathrm{~K}$ using the BOC power distribution. Although the average outlet temperature is the same for three different axial power distributions, the higher power rate at the outlet can lead to higher temperature gradients that cause higher maximum fuel temperature. In summary, without using a proper axial power profile from high fidelity tool calculation, the maximum fuel could be under-estimated by more than $100 \mathrm{~K}$ in this case. It also indicates that the maximum fuel temperature is quite sensitive to the power distribution. 


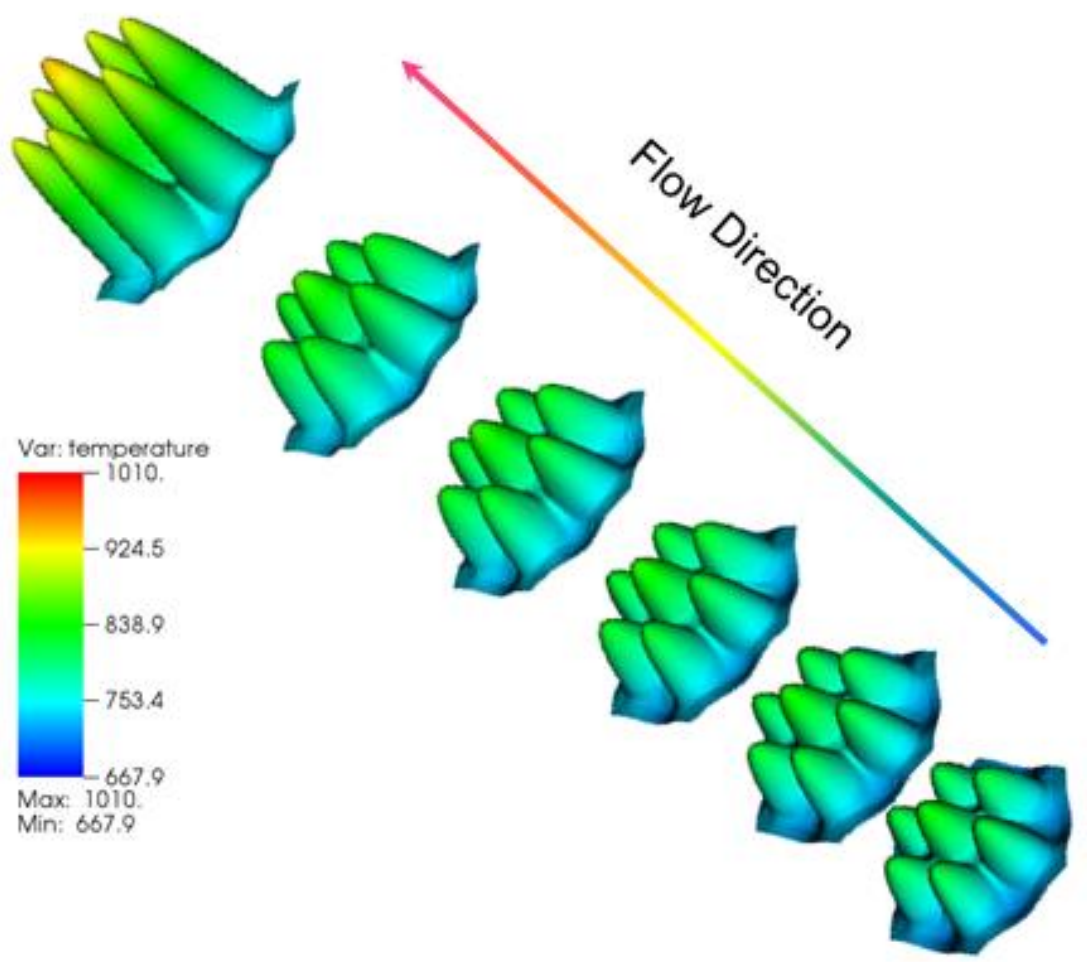

Figure 4.4.1.2 Temperature distributions at different Elevations (4.0P, 4.2P, 4.4P, 4.6P, 4.8P, 5.0P) in 7-pin bare rod bundle using PROTEUS power distribution 

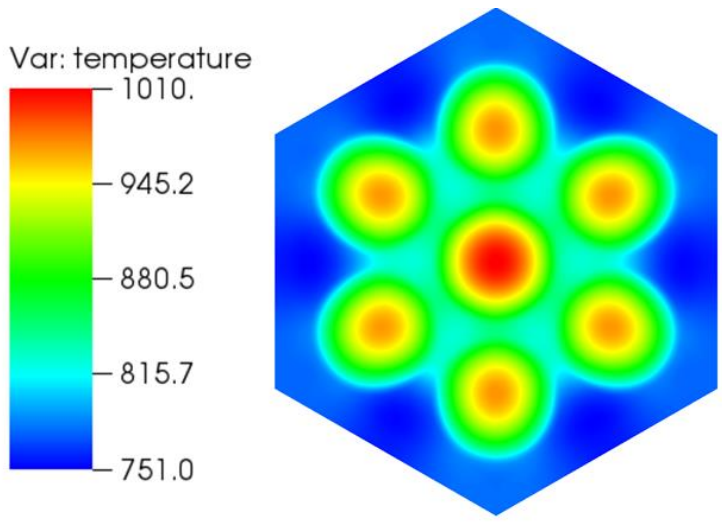

a) Power distribution from PROTEUS
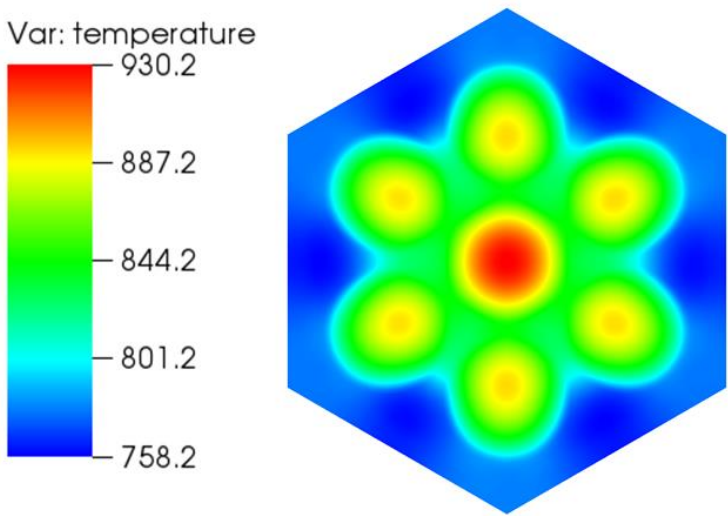

b) Uniform power distribution
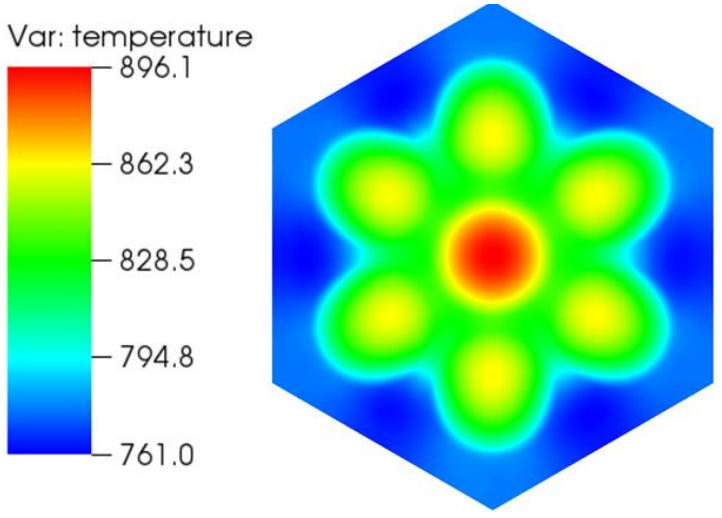

c) Power distribution from core average power at BOC

Figure 4.4.1.3 Outlet temperature distributions with different axial power distribution in 7pin bare rod bundle

It is beneficial to analyze the detailed maximum temperature in different sub-channels and rods according to the numbering scheme shown in Figure 4.4.1.4. Rod 1 is the center rod which is surrounded by six sub-channels, labelled 1-6. Rods 2 through 7 are located in the second ring of 
the assembly. Only sub-channels 1-6 as shown in the figure are analyzed because other subchannels are located at the edge of the assembly and experience a sharp temperature drop.

The maximum temperature for the different sub-channels and rods are shown in 4.4.1.5. For the same axial power profile, the maximum temperatures in all the sub-channels are similar. The difference is within $2{ }^{\circ} \mathrm{C}$. The slight asymmetry of the maximum temperature is caused by the mixing introduced by the coherent structure in the fluid flow. The cladding and fuel maximum temperature of rod 1 is obviously higher than other rods that neighbor the edge sub-channels which are much cooler than the center sub-channels.

The case using the axial power distribution from PROTEUS has higher maximum temperature than the cases using the uniform or core average BOC power distributions. While the average bulk temperature in the core is the same for all power profiles (due to same average power production across the core), the higher power rate at the outlet in the PROTEUS distribution leads to higher heat flux at that location. The increased heat flux must be associated with an increased temperature gradient and therefore higher outlet temperature. However, the detailed maximum temperatures in different sub-channels and rods have similar trends despite the different axial power distributions. Therefore the axial power distribution only affects the magnitude of the maximum temperatures, and the overall trends are insensitive to the axial power shape.

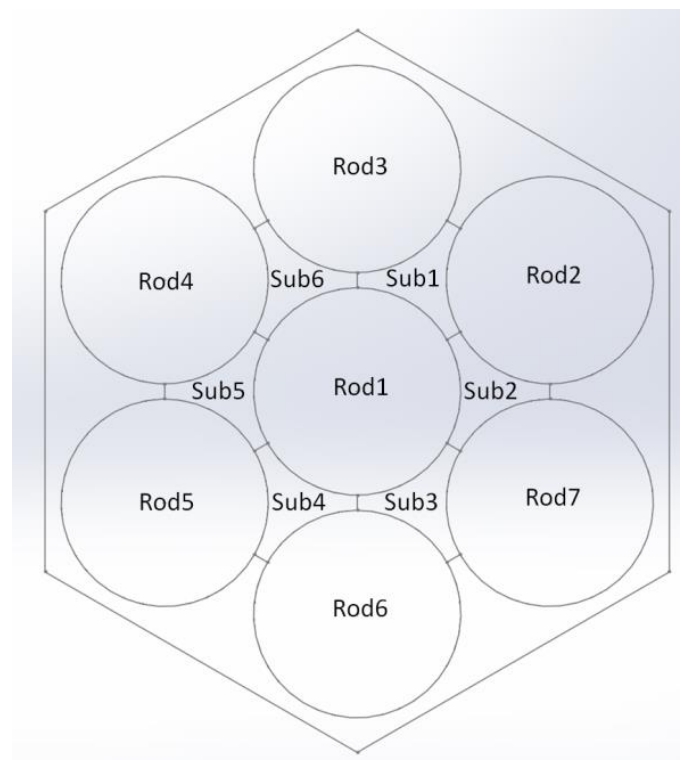

Figure 4.4.1.4 Numbering scheme for sub-channels and rods in 7-pin bare rod bundle 


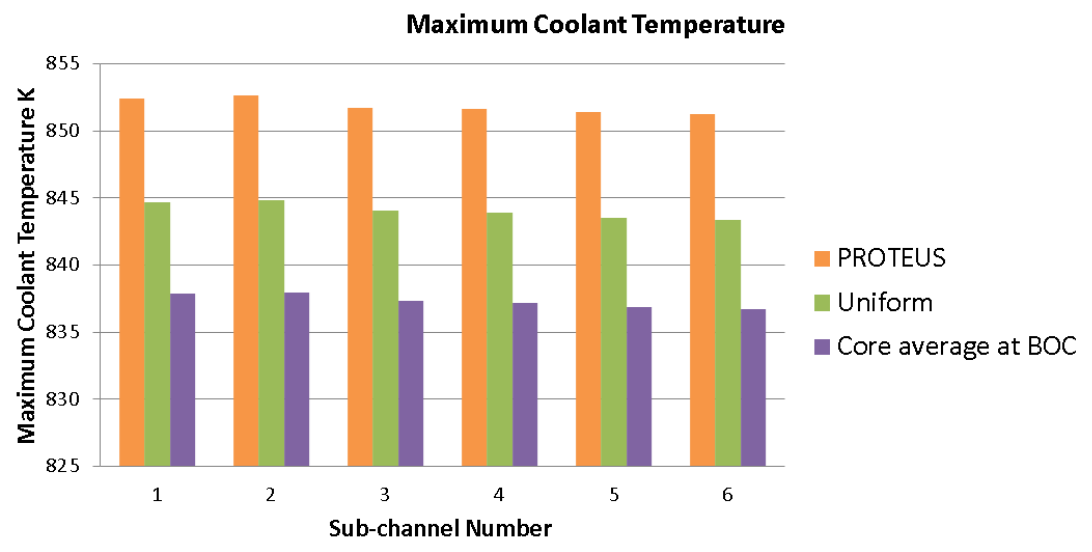

a) Maximum Coolant Temperature in different Sub-channels

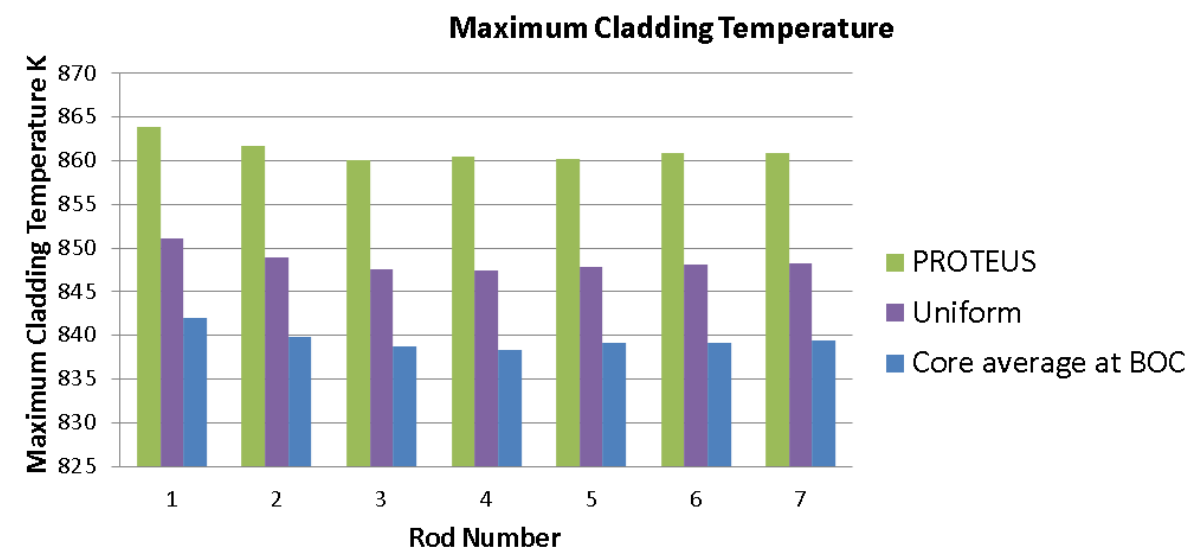

b) Maximum Cladding Temperature in Different Rods

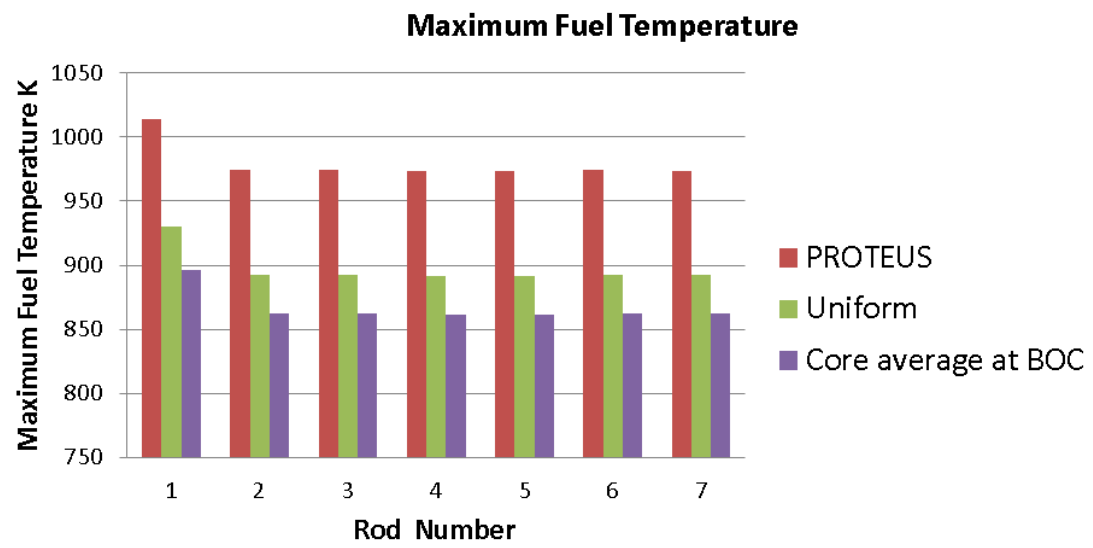

c) Maximum Fuel Temperature in Different Rods

Figure 4.4.1.5 Maximum temperature for different sub-channels and rods in 7-pin bare rod bundle 


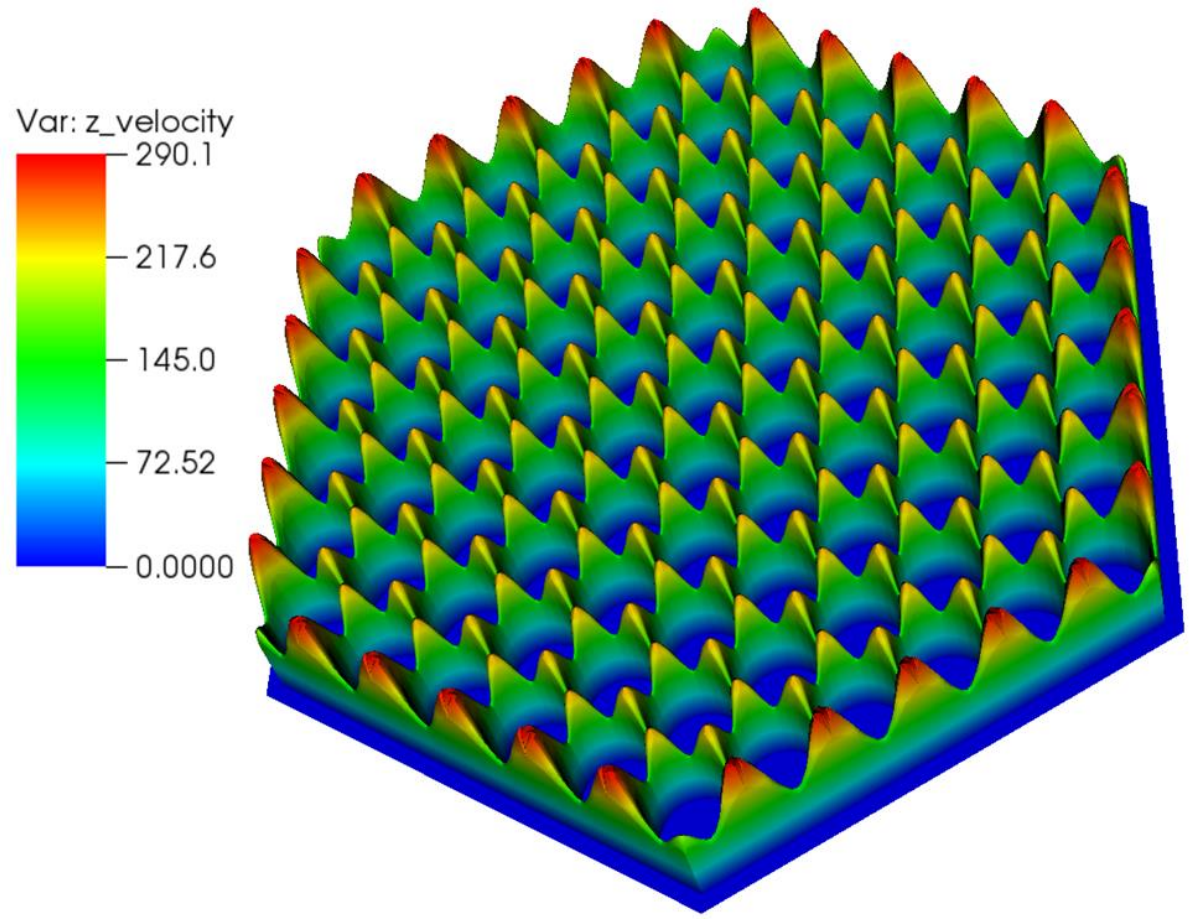

a) Velocity Distribution

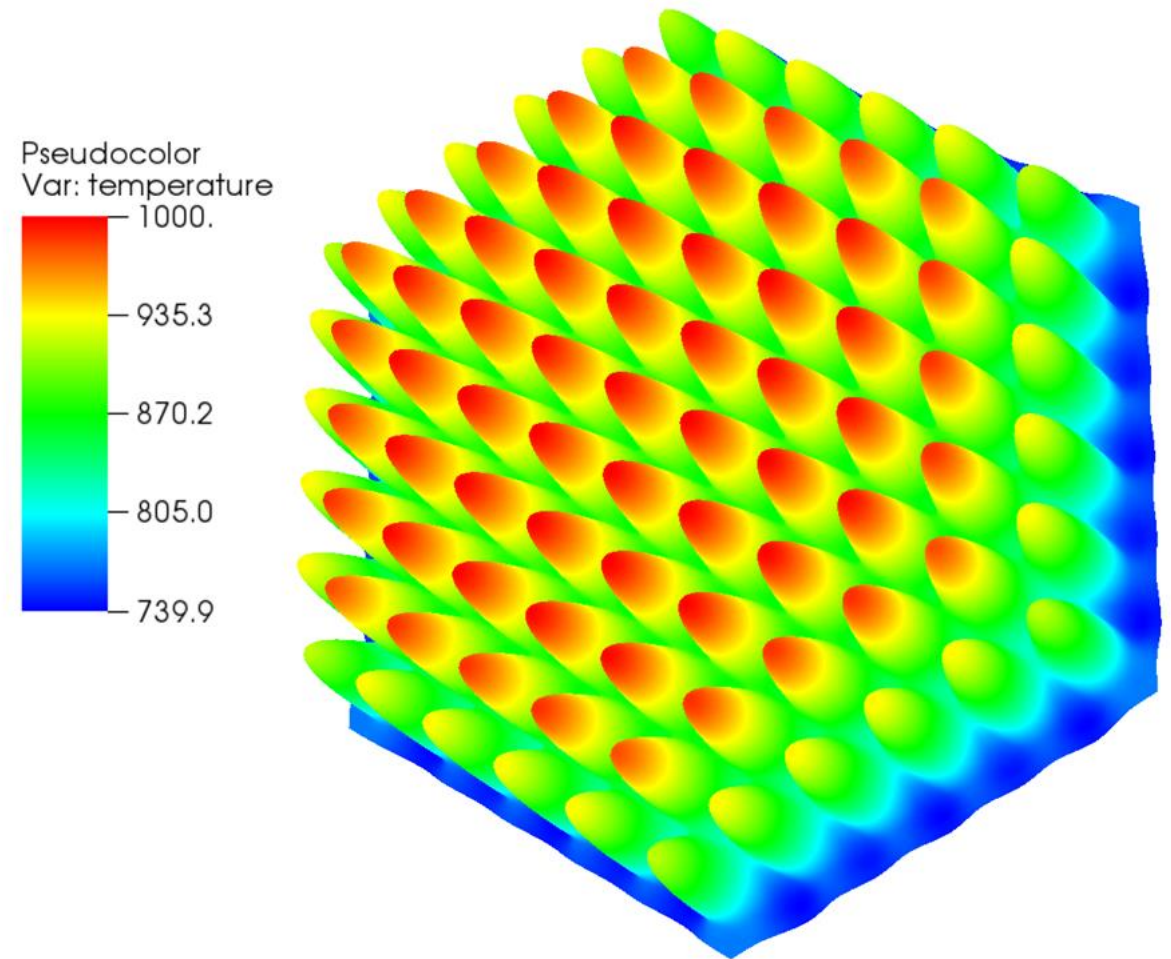

a) Temperature Distribution

Figure 4.4.1.6 Outlet velocity and temperature distribution in 91-pin bare rod bundle 
The velocity and temperature distribution at the outlet in the 91-pin bare rod bundle are shown in Figure 4.4.1.6. The velocity in the edge sub-channels is obviously larger than that in other subchannels due to larger hydraulic diameter. The temperature in the edge sub-channels is colder mainly because of two factors: increased velocity as shown, and lower volumetric heat flux in the edge zone.

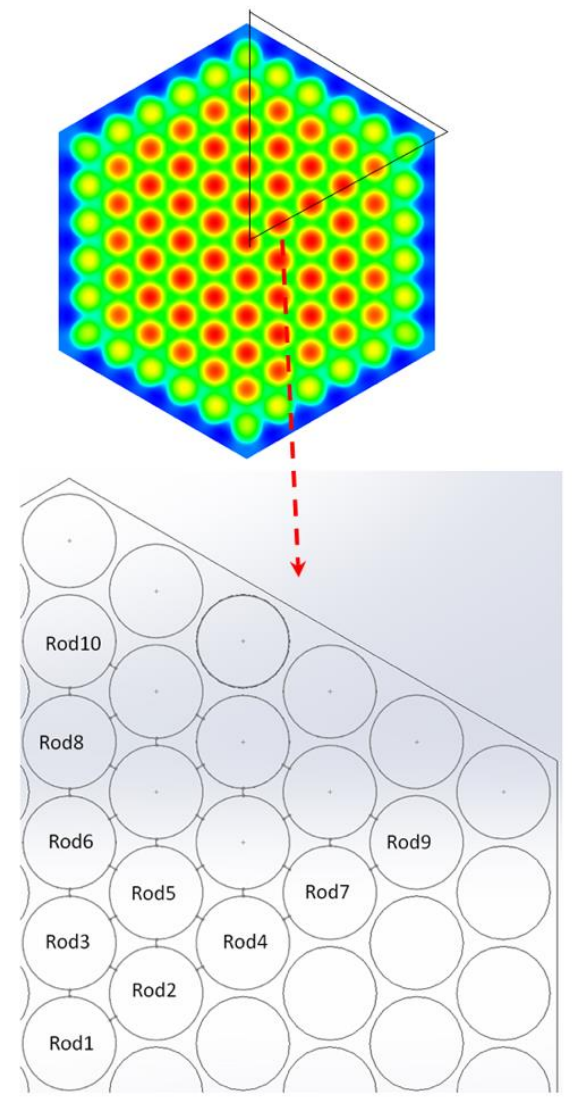

Figure 4.4.1.7 Rod numbering scheme in 91-pin bare rod bundle

In order to better understand the temperature distribution in a 91-pin bare rod bundle, the maximum fuel and cladding temperature are analyzed. Based on the analysis of the 7-pin bare rod bundle, the temperature is quite homogeneous in each ring. Only $1 / 6^{\text {th }}$ of 91 -pin assembly will be examined. The numbering scheme of rods in 91-pin bare rod bundle is shown in Figure 4.4.1.7. Rod 1 is the center pin. Rod 9 and Rod 10 are located at the $5^{\text {th }}$ ring of the assembly. 


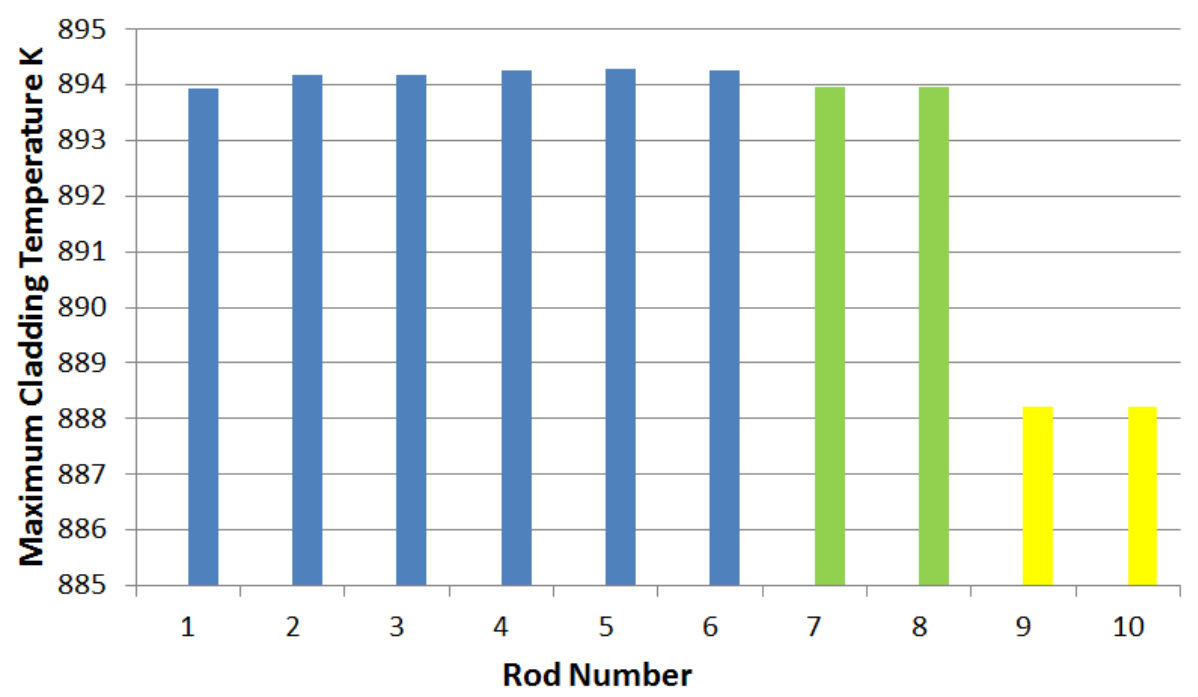

a) Maximum cladding Temperature

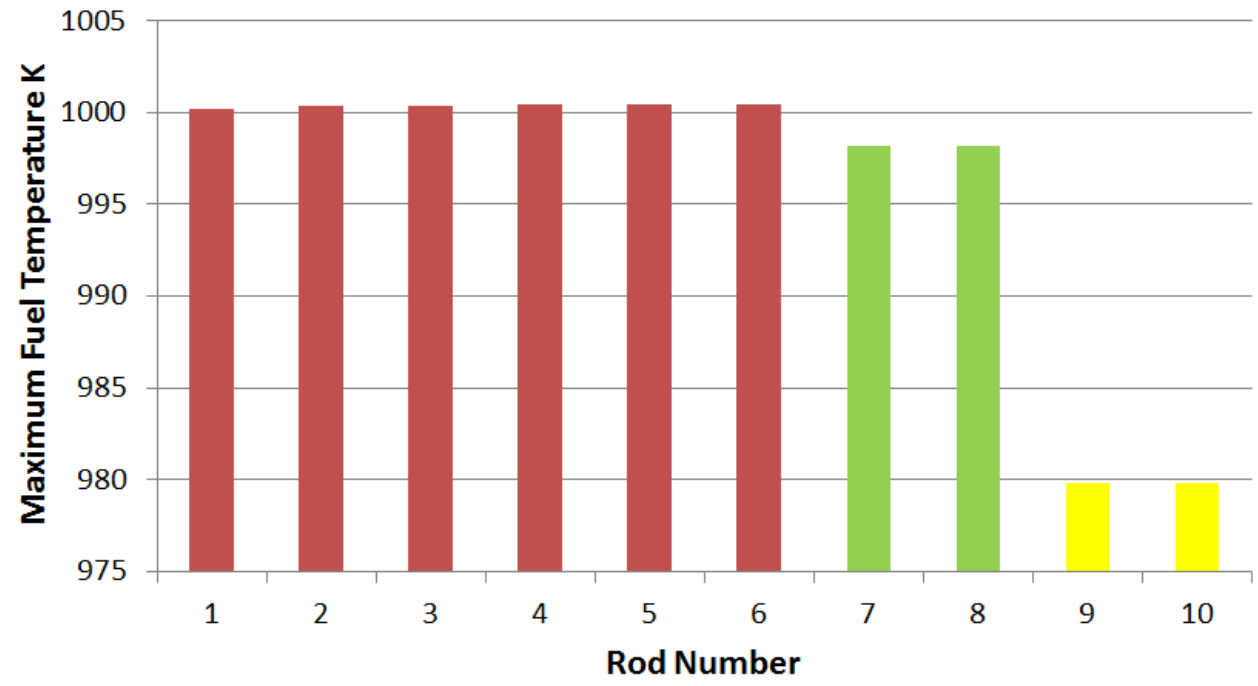

b) Maximum Fuel Temperature

Figure 4.4.1.8 Maximum temperature for different rods in 91-pin bare rod bundle

Figure 4.4.1.8 shows the maximum temperature for different rods in 91-pin bare rod bundle. Given that heat conduction is the only heat transfer mechanism between cladding and fuel, the trend of maximum temperature for fuel and cladding is similar. Within the $3^{\text {rd }}$ ring of the assembly, the maximum temperature is quite homogenous. The maximum temperature begins to drop at the $4^{\text {th }}$ ring. At 5th ring, the maximum temperature drops significantly because it is right next to the peripheral layer of the assembly. For the first 3 rings, $4^{\text {th }}$ and $5^{\text {th }}$ ring act as an insulation from the coolest zone of the assembly. That reveals the mechanism of why the hot spots always appear within the first 3 rings (for AFR-100) of the assembly. 


\subsubsection{Wire Wrap Rod Bundle Results}

The simulations on 7-pin and 91-pin wire wrap rod bundles are performed in Nek5000 to investigate the effect of the modeling the wire wrap explicitly as opposed to the bare bundle. The velocity distributions for both 91-pin bare rod bundle and wire wrap rod bundle are shown in Figure 4.4.2.1. Obvious differences in the velocity distribution are observed due to the wire effect. Strong inter-channel mixing is introduced by the wire, which affects not only the lateral velocity but also the stream wise velocity.

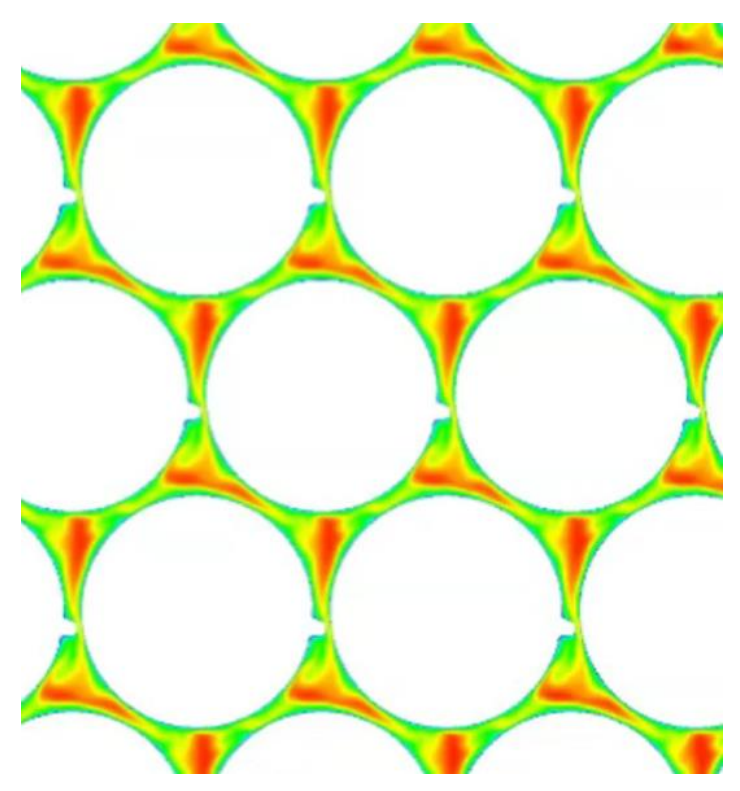

a) wire wrap rod bundle

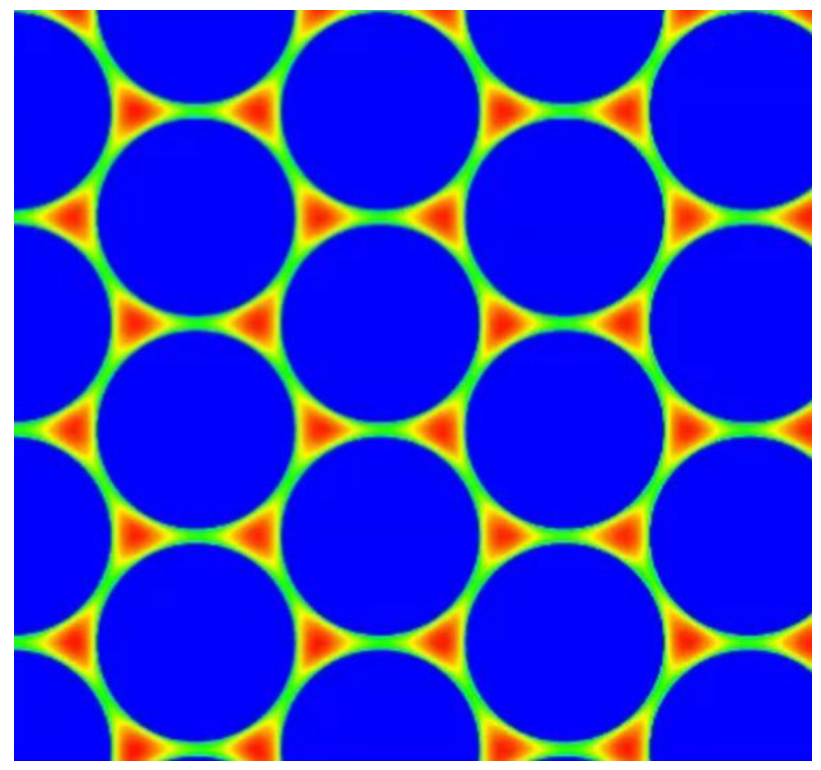

b) bare rod bundle

Figure 4.4.2.1 Comparison of outlet velocity distribution between 91-pin bare rod bundle and 91-pin wire wrap rod bundle

The maximum coolant temperature in different sub-channels for 7-pin and 91-pin are shown in Figure 4.4.2.2 and Figure 4.4.2.3. Due to stronger inter-channel mixing in the wire wrap rod bundle, the the maximum coolant temperature in different sub-channels deviates more than that in the bare rod bundle. However, the hottest sub-channel has similar maximum coolant temperature in both bare and wire wrap rod bundles. This finding reveals that modeling the wire wrap has a limited impact for HCF calculations. Bare rod bundle simulation can accurately capture the maximum temperature in spite of missing the precise local temperature distribution. Given that the bare rod bundle calculation is much cheaper, the bare rod bundle model is a more practical and efficient model for such calculations. In addition, the wire wrap model can introduce extra error due to simplifying the contact point between the wire and the rod, without taking into account the conjugate heat transfer and by using the periodic boundary condition. The maximum outer cladding temperature deviation between 91-pin bare rod bundle and 91-pin wire wrap rod bundle is about 2 ${ }^{\circ} \mathrm{C}$. In addition, the outer cladding maximum temperature appears on Rod 3 and Rod 5 in the wire wrap rod bundle, which is also well captured by bare rod bundle model. 


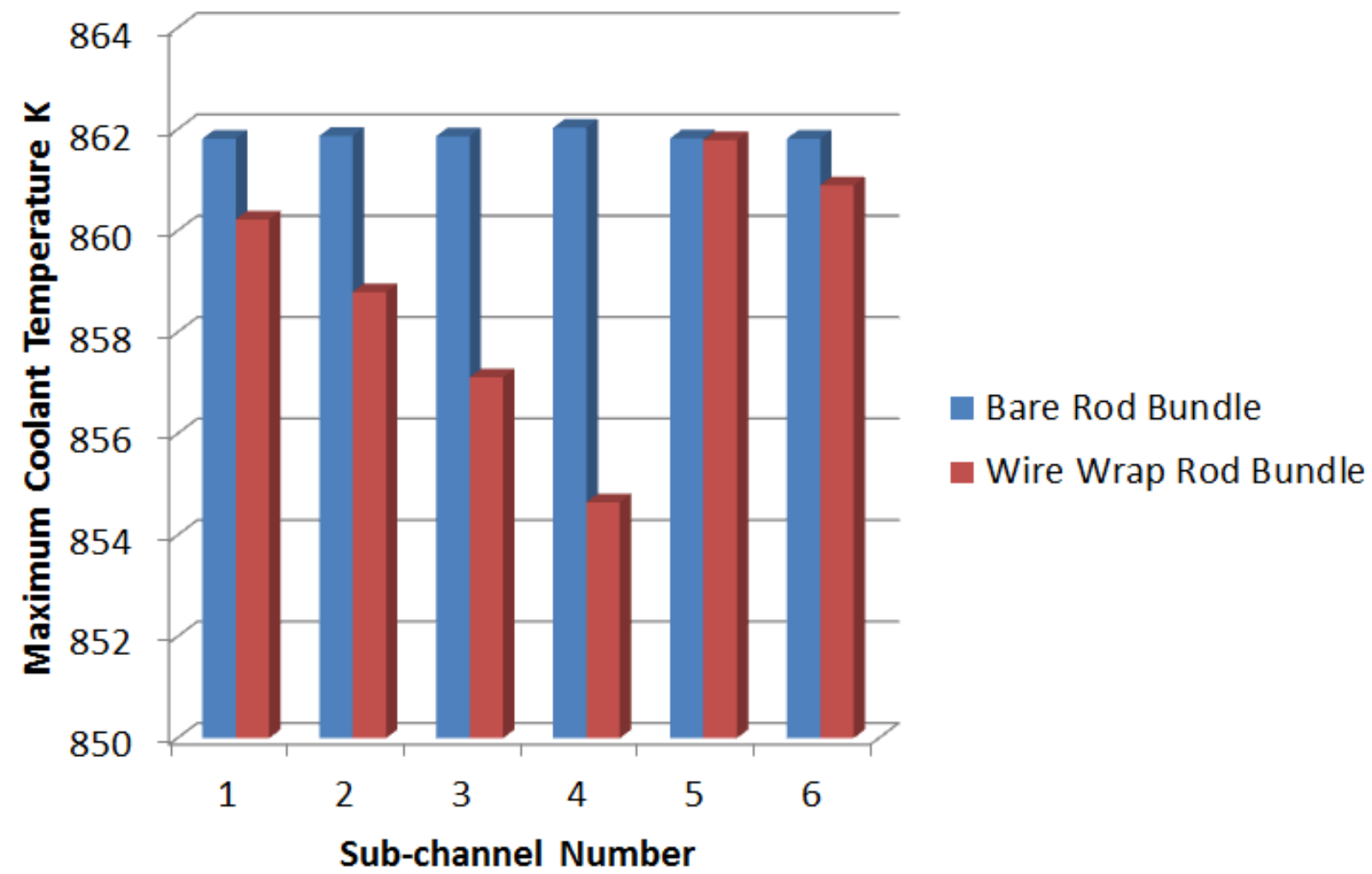

Figure 4.4.2.2 Sub-channel maximum coolant temperature between 7-pin bare rod bundle and 7-pin wire wrap rod bundle

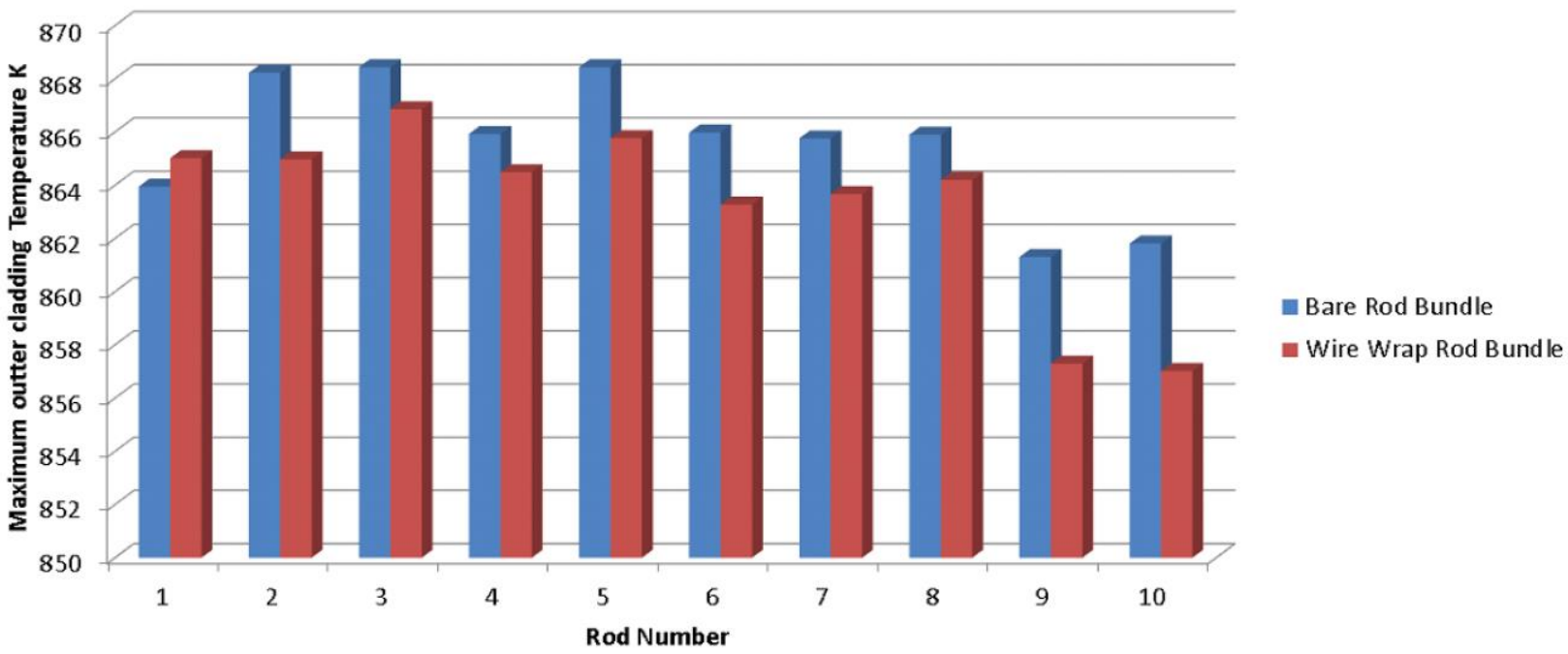

Figure 4.4.2.3 Maximum outer cladding temperature between 91-pin bare rod bundle and 91-pin wire wrap rod bundle 
To calculate the hot channel factor due to reversed wire orientation, the explicit modeling of wire wrap is necessary. However, the generation of a pure hexahedral mesh for a wire wrapped rod bundle with different wire orientations is challenging. Given the complexity of the geometric feature, the HCF calculation for wire orientation was performed in only the 7-pin wire wrapped rod bundle. An innovative tet-to-hex meshing strategy is applied in this study. Figure 4.4.2.4 shows the work flow of this strategy, which begins with a tetrahedral mesh. Since the tetrahedral elements can best represent the complex geometry, the automated mesh generation capability in ICEM CFD 18.2 was employed to build the pure tetrahedral mesh for the wire wrap rod bundle with the center pin wire wrap oriented in the opposite direction. Then, the tetrahedral mesh is converted to hexahedral mesh to adapt the mesh to Nek5000. Finally, mesh extrusion is applied to build boundary layers for better simulation of the flow near the wall.

In order to provide a code-to-code verification, the simulation for wire wrap with different wire orientations is also performed with STAR-CCM+. The velocity distributions from Nek5000 and STAR-CCM+ are presented in Figure 4.4.2.5 and show good agreement. Some deviation is caused by different numerical methods used in these codes (URANS for Nek5000 and RANS for STAR$\mathrm{CCM}+$ ).

Both velocity and temperature for wire wrap bundle with same and different wire orientation is shown in Figure 4.4.2.6 and Figure 4.4.2.7. Reversal of the wire orientation in the center pin disturbs the flow pattern in the vicinity of the center pin and thus the temperature distribution. As seen from the figure, it changes the location of the hot spot rather than the temperature value of the hot spot. The effects incurred by reversed wire wrap in a pin are well captured by the SHARP model. This hot channel factor could not be well estimated by other tools previously. 


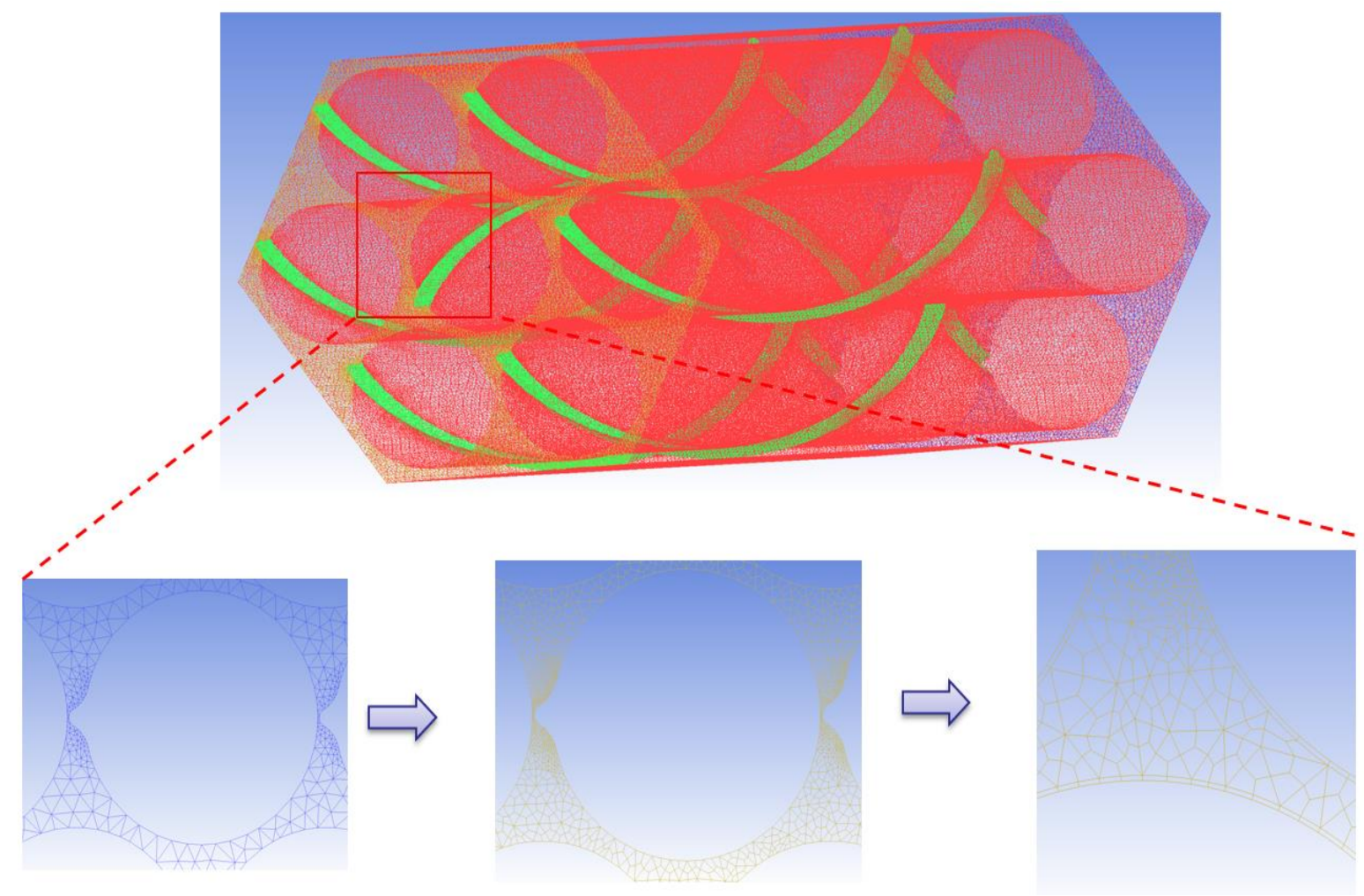

Figure 4.4.2.4 Workflow of tet-to-hex meshing strategy

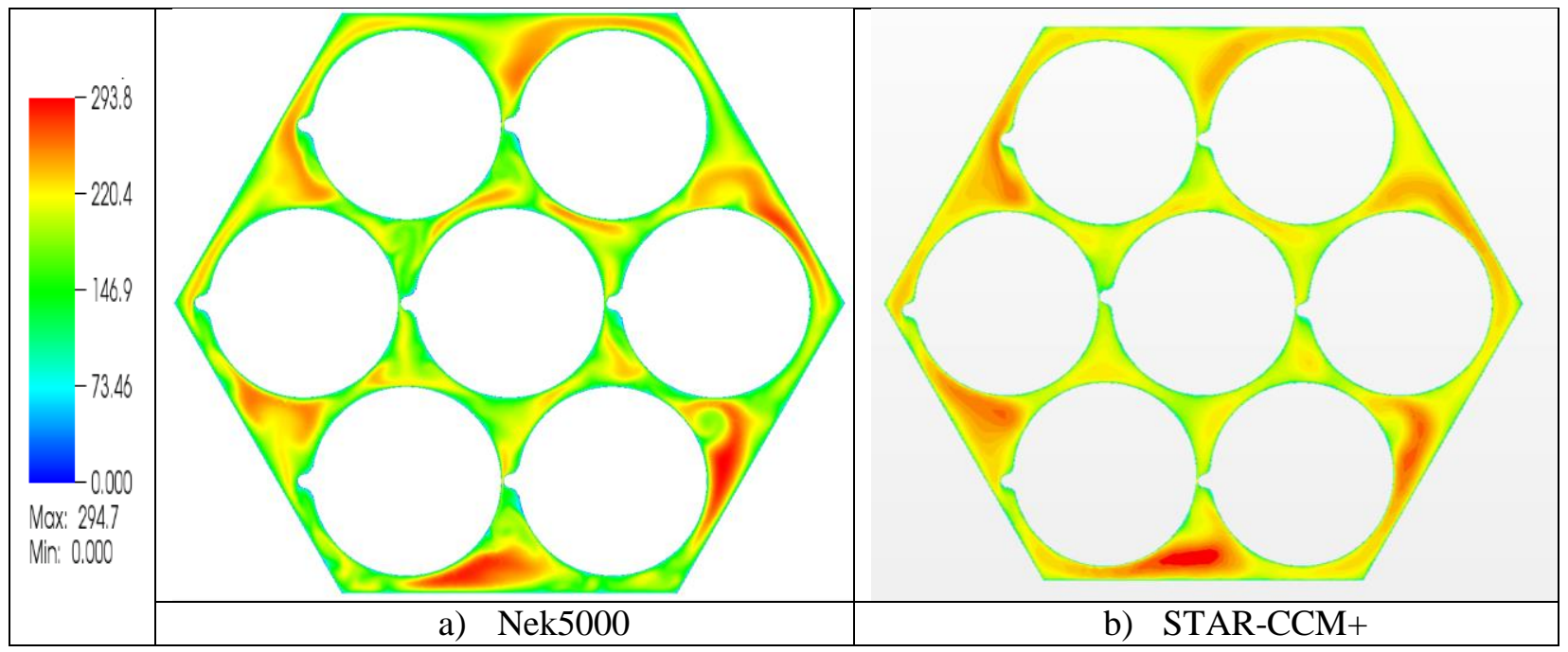

Figure 4.4.2.5 Velocity distribution with different wire orientation from Nek5000 and STARCCM+ 


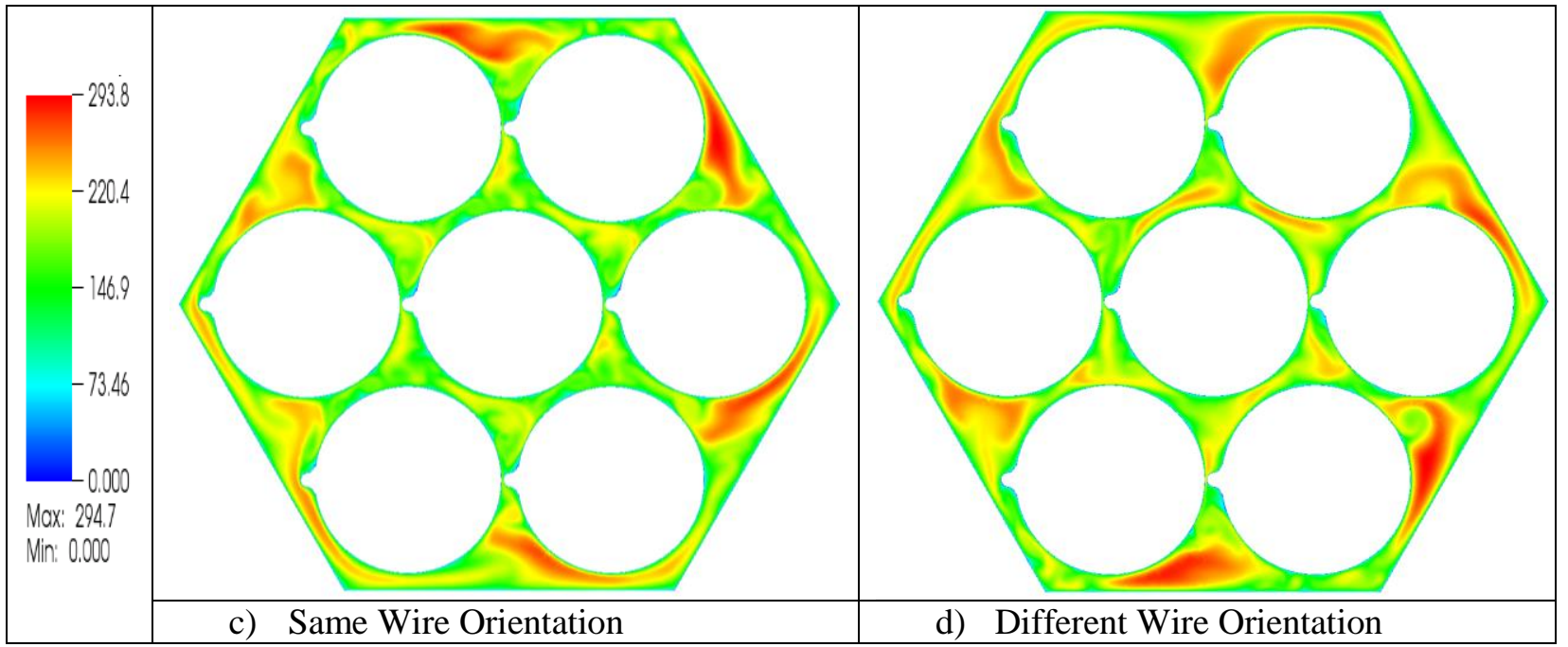

Figure 4.4.2.6 Comparison of outlet velocity distribution with same and different wire orientation

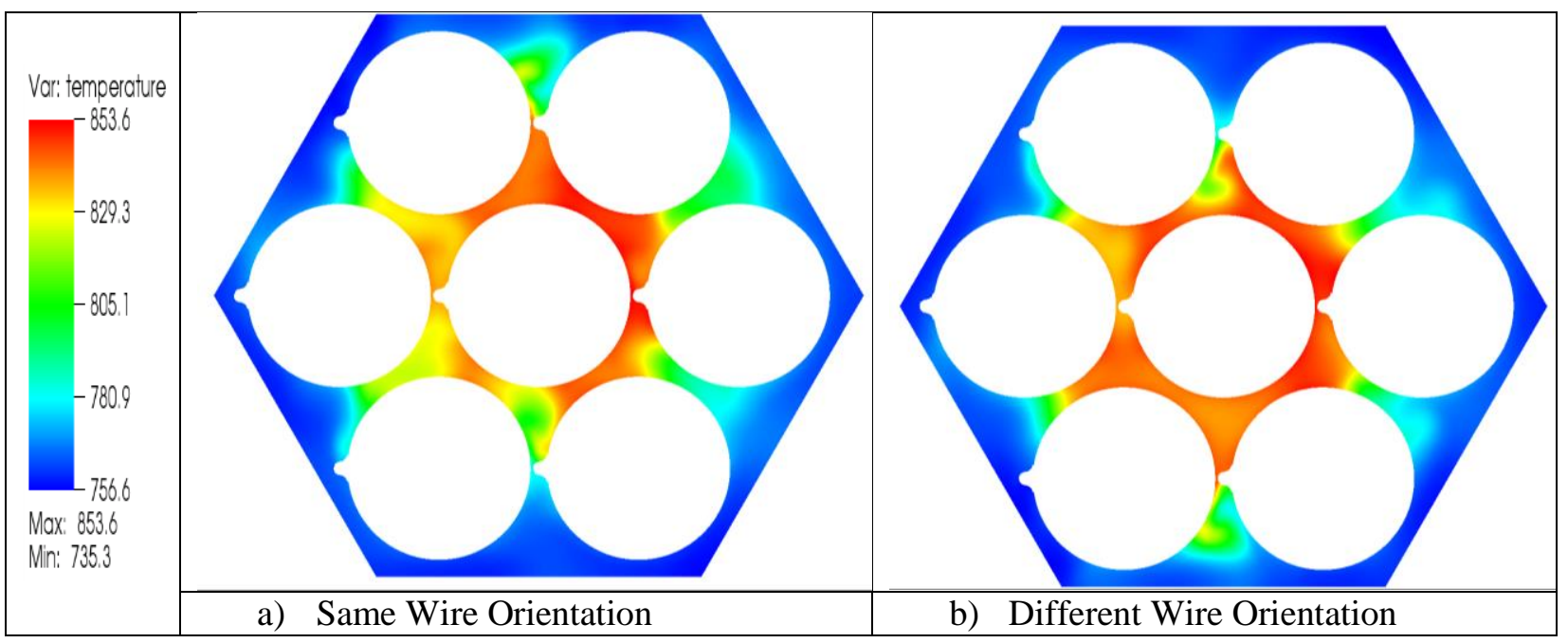

Figure 4.4.2.7 Comparison of outlet temperature distribution with same and different wire orientation

\subsection{Estimation of Hot Channel Factors}

The previous sections describe the neutronics and thermal hydraulic models for the nominal condition. Since the perturbations considered here are usually not big enough to change the flow and heat transfer behavior significantly, the study associated with the uncertainties is investigated based on the computational models (meshes, etc.) built for the nominal condition.

The peak cladding temperature HCFs due to perturbed coolant density and coolant specific heat are calculated in both the bare rod bundle and the wire wrap rod bundle, and listed in Table 4.5.1. 
The calculated hot channel factors of the peak cladding temperature induced by the uncertainties in the coolant properties are not sensitive to the number of pins in the bundle configurations, and the results from bare rod bundle configuration are more conservative than those from wire-wrap rod bundle configuration. Even though analysis of a smaller pin bundle may be sufficiently accurate for HCF calculations, in this study, we chose to use as ideal model as possible to demonstrate the capability of SHARP for HCF calculations. Therefore, the 91-pin bare bundle configurations were utilized to calculate further HCFs.

Table 4.5.1 Hot channel factors in bare rod bundle and wire wrap rod bundle

\begin{tabular}{|c|c|c|c|c|c|}
\hline \multirow{2}{*}{} & \multirow{2}{*}{ Uncertainties } & \multicolumn{2}{|c|}{ 7-pin } & \multicolumn{2}{c|}{ 91-pin } \\
\cline { 3 - 6 } & $\begin{array}{c}\text { Bare rod } \\
\text { bundle }\end{array}$ & $\begin{array}{c}\text { Wire wrap } \\
\text { rod bundle }\end{array}$ & $\begin{array}{c}\text { Bare rod } \\
\text { bundle }\end{array}$ & $\begin{array}{c}\text { Wire wrap } \\
\text { rod bundle }\end{array}$ \\
\hline $\begin{array}{c}\text { Coolant } \\
\text { Density }\end{array}$ & $\pm 0.5 \%$ & 1.001 & 1.0002 & 1.001 & 1.0002 \\
\hline $\begin{array}{c}\text { Coolant } \\
\text { Specific Heat }\end{array}$ & $\pm 3 \%$ & 1.016 & 1.016 & 1.016 & 1.016 \\
\hline
\end{tabular}

Several perturbed conditions (due to some physical uncertainty, manufacturing tolerance, or modeling approximation) were investigated to assess the increase in the peak temperatures.. The results are listed in Table 4.5.2. All hot channel factors were computed using the 91-pin model except for the wire wrap vs. bundle and reverse wire wrap orientation HCFs which used the 7-pin model. The detailed temperature data and equations used for each HCF calculation are described in Appendix E. The uncertainties or manufacturing tolerances and HCFs for EBR-II were obtained from Ref. [Ku 1994], which was developed for Mark-V fuels. Compared to the HCFs for EBR-II Mark-V fuel, the newly estimated HCFs using the SHARP toolkit are reduced in general due to the reductions of uncertainties in modeling and simulation. For instance, in the EBR-II, the uncertainty of fuel thermal conductivity was considered as the HCF of fuel centerline temperature by assuming that the fuel centerline temperature is proportional to the fuel thermal conductivity. However, the calculated variation of the peak fuel centerline temperature was slightly smaller than the uncertainty of fuel thermal conductivity. Moreover, the hot channel factor from manufacturing fissile maldistribution was evaluated based on a stochastic technique. Thirty distributions of the fissile enrichment in the peak assembly were randomly selected and simulated by the PROTEUS code, and the power distribution for a bounding case was provided to Nek5000 for a temperature calculation.

The temperature data used to calculate the various hot channel factors is included in Table 4.5.3. The corresponding equations for each hot channel factor are listed in Table 4.5.4. As seen in Table 4.5.2, the largest reduction in HCF was seen by using the SHARP tools to stochastically sample the fissile maldistribution occurring from manufacturing uncertainties. This large reduction can be explained by understanding that the legacy value assumed the worst case scenario: the center pin 
was manufactured with the largest possible deviation. This case is extremely likely even across numerous assemblies, and therefore the legacy value is overconservative. While the wire wrap calculations are extremely promising, some further work is recommended to analyze the impact of geometric modeling assumptions at the wire wrap-cladding interface. Overall, the results obtained with PROTEUS and Nek5000 show consistently improved results over legacy techniques. This procedure of computing HCF using high fidelity models shows promise for being repeated for any arbitrary reactor of choice, since these tools are targeted to solve many reactor types and geometries.

Table 4.5.2 Evaluation of hot channel factors for selected uncertainties

\begin{tabular}{|c|c|c|c|c|c|c|c|}
\hline \multirow[t]{2}{*}{ HCF } & \multirow{2}{*}{$\begin{array}{l}\text { Uncertainties } \\
\qquad(3 \sigma) \%\end{array}$} & \multicolumn{2}{|c|}{$\begin{array}{c}\text { Coolant HCF } \\
\text { (A) }\end{array}$} & \multicolumn{2}{|c|}{$\begin{array}{l}\text { Cladding HCF } \\
\text { (B) }\end{array}$} & \multicolumn{2}{|c|}{$\begin{array}{l}\text { Fuel HCF } \\
\text { (C) }\end{array}$} \\
\hline & & Legacy & SHARP & Legacy & SHARP & Legacy & SHARP \\
\hline Cladding Thickness & \pm 3 & & & $\begin{array}{c}1.03^{\sim} \\
1.05\end{array}$ & 1.018 & & \\
\hline $\begin{array}{l}\text { Cladding Thermal } \\
\text { Conductivity }\end{array}$ & \pm 7 & & & 1.088 & 1.082 & & \\
\hline $\begin{array}{l}\text { Fuel Thermal } \\
\text { Conductivity }\end{array}$ & \pm 25 & & & & & 1.25 & 1.226 \\
\hline Coolant Specific Heat & \pm 3 & 1.017 & 1.016 & & & & \\
\hline Coolant Density & \pm 0.5 & 1.016 & 1.001 & & & & \\
\hline $\begin{array}{l}\text { Fissile Mal- } \\
\text { distribution }\end{array}$ & \pm 6 & & & 1.06 & 1.036 & 1.06 & 1.016 \\
\hline $\begin{array}{l}\text { Cladding } \\
\text { Circumferential } \\
\text { Temperature } \\
\text { Variation }\end{array}$ & $\begin{array}{l}\text { (Wire wrap } \\
\text { vs bare } \\
\text { bundle) }\end{array}$ & 1.024 & 1.01 & & & & \\
\hline Wire Orientation & $\begin{array}{l}\text { (Reversed } \\
\text { wire } \\
\text { orientation in } \\
\text { center pin) }\end{array}$ & 1.01 & 1.003 & & & & \\
\hline
\end{tabular}


Table 4.5.3 Evaluation of temperature rises (degrees $\mathrm{K}$ ) in hot channels for selected uncertainties.

\begin{tabular}{|c|c|c|c|c|c|c|c|}
\hline \multirow{2}{*}{ HCF } & \multirow{2}{*}{$\begin{array}{c}\text { Uncertainties } \\
\text { (3s) \% }\end{array}$} & \multicolumn{2}{|c|}{$\begin{array}{c}\text { Coolant HCF } \\
\text { (A) }\end{array}$} & \multicolumn{2}{|c|}{$\begin{array}{l}\text { Cladding HCF } \\
\text { (B) }\end{array}$} & \multicolumn{2}{|c|}{$\begin{array}{l}\text { Fuel HCF } \\
\text { (C) }\end{array}$} \\
\hline & & $\Delta \mathrm{T}_{\mathrm{N}}$ & $\Delta T_{P}$ & $\Delta \mathrm{T}_{\mathrm{N}}$ & $\Delta T_{P}$ & $\Delta \mathrm{T}_{\mathrm{N}}$ & $\Delta T_{P}$ \\
\hline Cladding Thickness & \pm 3 & & & 6.67 & 6.79 & & \\
\hline $\begin{array}{l}\text { Cladding Thermal } \\
\text { Conductivity }\end{array}$ & \pm 7 & & & 6.67 & 7.22 & & \\
\hline $\begin{array}{l}\text { Fuel Thermal } \\
\text { Conductivity }\end{array}$ & \pm 25 & & & & & 142.32 & 174.48 \\
\hline $\begin{array}{l}\text { Coolant Specific } \\
\text { Heat }\end{array}$ & \pm 3 & 206.4 & 209.7 & & & & \\
\hline Coolant Density & \pm 0.5 & 206.4 & 206.6 & & & & \\
\hline $\begin{array}{l}\text { Fissile Mal- } \\
\text { distribution }\end{array}$ & \pm 6 & & & 6.67 & 6.91 & 142.32 & 144.6 \\
\hline $\begin{array}{l}\text { Cladding } \\
\text { Circumferential } \\
\text { Temperature } \\
\text { Variation }\end{array}$ & $\begin{array}{l}\text { (Wire wrap vs } \\
\text { bare bundle) }\end{array}$ & 204.4 & 206.4 & & & & \\
\hline Wire Orientation & $\begin{array}{l}\text { (Reversed } \\
\text { wire } \\
\text { orientation in } \\
\text { center pin) }\end{array}$ & 180.24 & 180.78 & & & & \\
\hline
\end{tabular}

N: Nominal P: Perturbed

Table 4.5.4 Equations to compute coolant, cladding, and fuel hot channel factors

\begin{tabular}{|c|c|}
\hline Coolant HCF (A) & $\begin{aligned} \mathrm{HCF}_{\mathrm{A}}= & \left(\mathrm{T}_{\text {max_coolant }}-\mathrm{T}_{\text {inlet_coolant }}\right)_{\text {perturbed }} / \\
& \left(\mathrm{T}_{\text {max_coolant }}-\mathrm{T}_{\text {inlet_coolant }}\right)_{\text {nominal }}\end{aligned}$ \\
\hline Cladding HCF (B) & $\begin{aligned} \mathrm{HCF}_{\mathrm{B}}= & \left(\mathrm{T}_{\text {cladding_innerwall }}-\mathrm{T}_{\text {cladding_outerwall }}\right) \text { perturbed } \\
& \left(T_{\text {cladding_innerwall }}-\mathrm{T}_{\text {cladding_outerwall }}\right) \text { nominal }\end{aligned}$ \\
\hline Fuel HCF (C) & $\begin{aligned} H C F_{c}= & \left(T_{\text {fuel_centerline }}-T_{\text {fuel_outerwall }}\right)_{\text {perturbed }} / \\
& \left(T_{\text {fuel_centerline}}-T_{\text {fuel_outerwall }}\right)_{\text {nominal }}\end{aligned}$ \\
\hline
\end{tabular}




\section{Zoom Capability of the SHARP Toolkit}

The SHARP zoom concept is a hybrid core modeling and simulation scheme whereby coarseand-homogeneous meshes and fine-and-heterogeneous meshes are combined: i.e., most assemblies are represented using the conventional homogenized meshes, but a few assemblies of interest are represented with pin-by-pin heterogeneous meshes in order to gain accurate local information. The key idea behind SHARP zoom is to significantly lower the computational expense while preserving high fidelity in a region of interest. By demonstrating the feasibility of the SHARP zoom concept, the computational expense of using the high fidelity SHARP tools will be significantly reduced, and the usability of the SHARP toolkit will be improved.

\subsection{Application of Zoom Capability to AFR-100 Peak Temperature Calculations}

The SHARP zoom concept is demonstrated for the full core AFR-100 design by selecting the assembly with peak cladding temperature as the focal assembly to be represented heterogeneously. Figure 5.1.1 (left) illustrates the peak cladding temperatures (BOC and EOC) in the assemblies as calculated by legacy tools in a previous analysis, which is in an outer core fuel assembly.

For zooming calculations, a full core PROTEUS neutronics simulation is required to provide accurate neutron current distributions at the surfaces of the focal assembly (i.e., boundary condition). Before demonstrating the zooming capability, a sensitivity analysis was conducted in this study to answer to the question of "how many neighboring assemblies should be explicitly modeled in the PROTEUS calculations to achieve accurate solutions?" Three core models are considered in this study: homogeneous, single-heterogeneous, and seven-heterogeneous assembly models.

In the homogeneous assembly model, all assemblies are modeled homogeneously. In the singleheterogeneous calculation, the single focal assembly is modeled with pin-by-pin explicit geometry, and the other assemblies are modeled with a conventional homogenized assembly approach. In the seven-heterogeneous assembly model, the focal assembly and the surrounding six assemblies are modeled pin-by-pin, and the remainder of the core is homogenized.

The single-heterogeneous assembly model is depicted in Figure 5.1.1. Considering the neutron diffusion length is typically shorter than the assembly pitch in an SFR, it is expected that the flux distributions in the focal assembly from seven-heterogeneous assembly model would be accurate. If the single-heterogeneous assembly model yields similar results to the seven-heterogeneous assembly model, the SHARP zoom concept is promising as it indicates that local distributions are not sensitive to modeling approximations outside the assembly of interest: i.e., the singleheterogeneous model of the focal assembly is sufficient for zooming calculations. In contrast to the PROTEUS calculations, full core simulations are not needed for the Nek5000 calculations because there is no cross flow between assemblies and the thermal-hydraulic parameters in each assembly are isolated by duct. But in order to decide the appropriate number of heterogeneous assemblies in 
the Nek5000 calculations, sensitivity calculations were also conducted with a single heterogeneous assembly model and seven heterogeneous assembly model. The detailed results of the sensitivity analyses are provided in Sections 5.2 and 5.3.
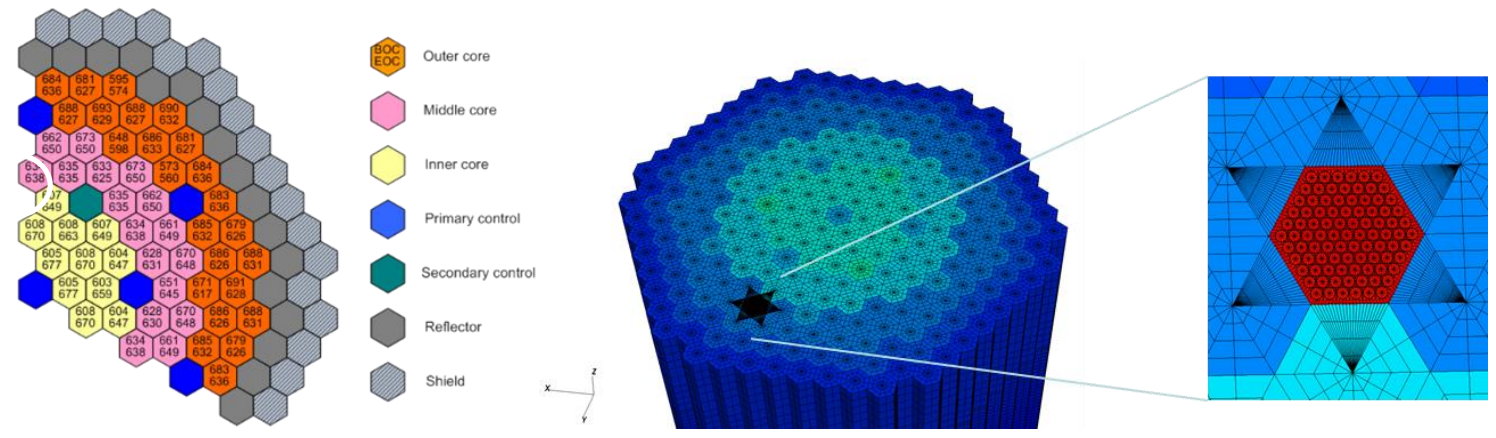

Figure 5.1.1 AFR-100 modeling for SHARP zoom calculation

\subsection{Neutronics Calculations}

PROTEUS-SN was selected as the neutronics solver for the zooming calculations because it offers significant computational advantages over PROTEUS-MOCEX for large core problems with significant homogenization. This section describes the details of the neutronics calculations.

\subsubsection{Neutronics Meshes}

Three 3-D linear finite element neutronics meshes were prepared for the zooming calculations (see Table 5.2.1.1 for a summary). The homogeneous assembly mesh has 0.938 million vertices, the single-heterogeneous assembly mesh has 1.27 million vertices, and the seven-heterogeneous mesh has 3.06 million vertices. By extrapolation, a full core heterogeneous mesh (265 assemblies) would require roughly 80 million vertices. Due to the excellent scalability of PROTEUS-SN, the computational cost is directly related to the mesh size. Since the largest mesh here is only $4 \%$ of the size of a fully explicit core mesh, the computational expense is likely to be a similar fraction of a fully explicit core calculation. Images of the three meshes follow in Figure 5.2.1.1 - Figure 5.2.1.4. Notably, the explicitly modeled assemblies require many more finite elements to represent the pin features (cladding, fuel). In order to maintain a conformal mesh, sectors of the homogeneous assemblies neighboring the pin-by-pin assemblies are also required to have finer meshes, even though there are no geometry details in those assemblies.

Table 5.2.1.1 Neutronics meshes used in zooming calculations. 


\begin{tabular}{|l|c|}
\hline Mesh & Number of Vertices \\
\hline Homogeneous & $0.938 \mathrm{M}$ \\
\hline Single-Heterogeneous & $1.27 \mathrm{M}$ \\
\hline Seven-Heterogeneous & $3.06 \mathrm{M}$ \\
\hline
\end{tabular}
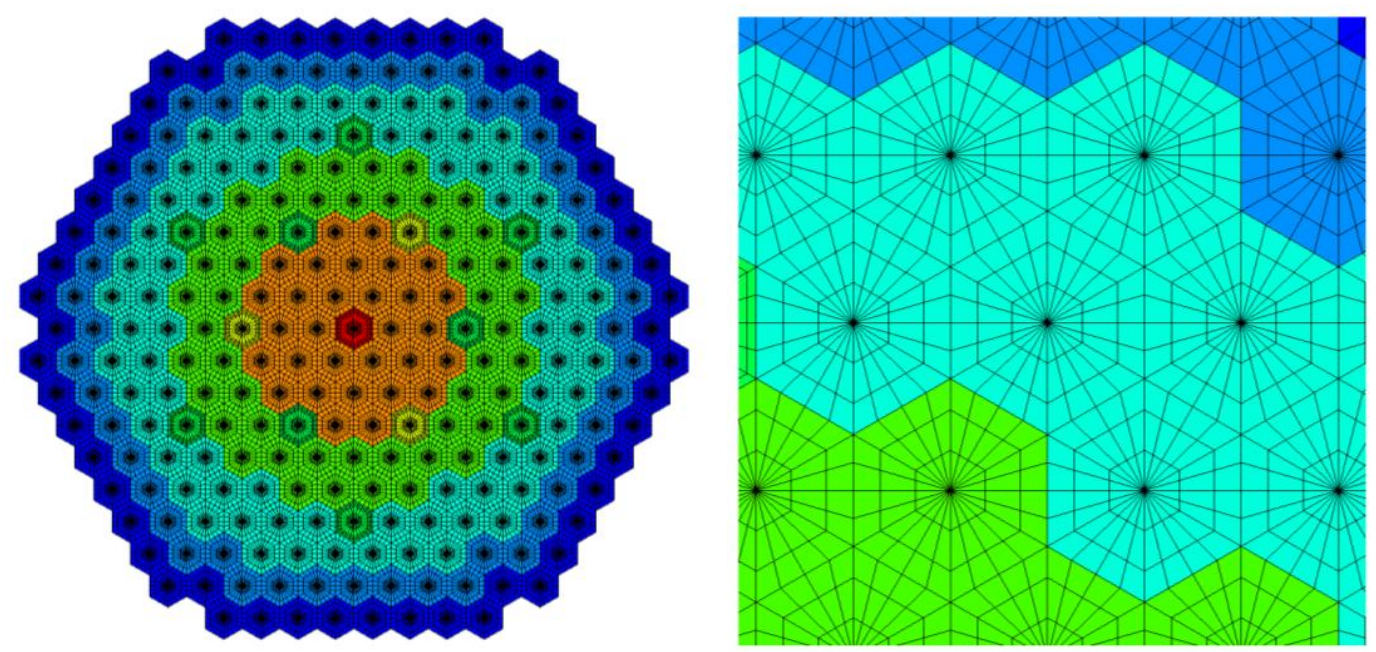

Figure 5.2.1.1 Homogeneous AFR-100 mesh showing focal assembly in center of right image.
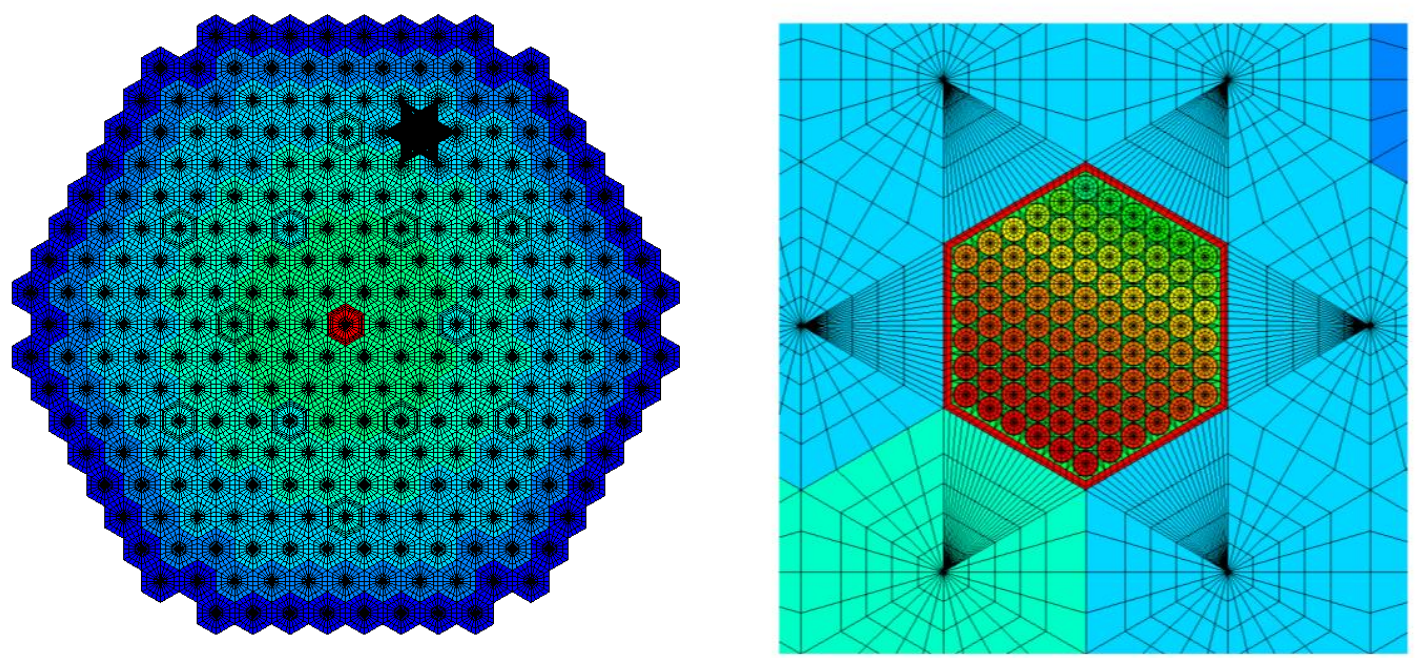

Figure 5.2.1.2 Single-heterogeneous assembly AFR-100 mesh showing focal assembly in center of right image. 

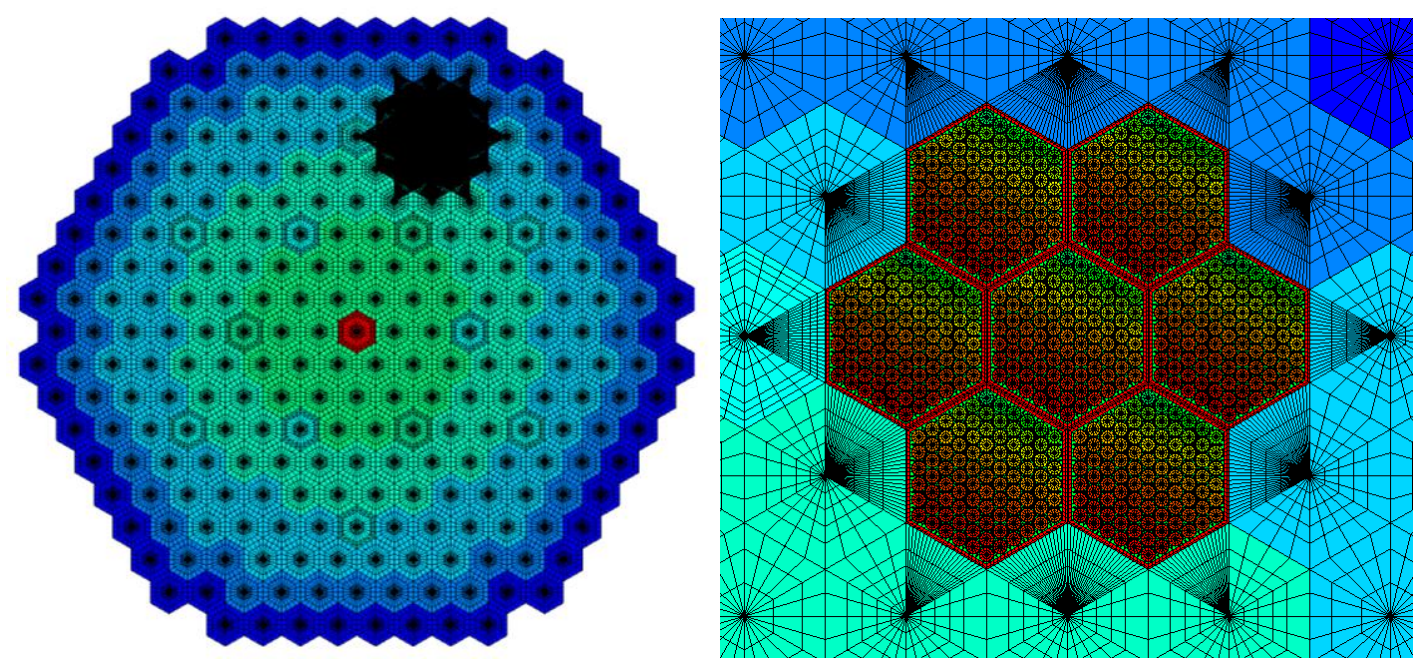

Figure 5.2.1.3 Seven-heterogeneous assembly AFR-100 mesh showing focal assembly in center of right image.

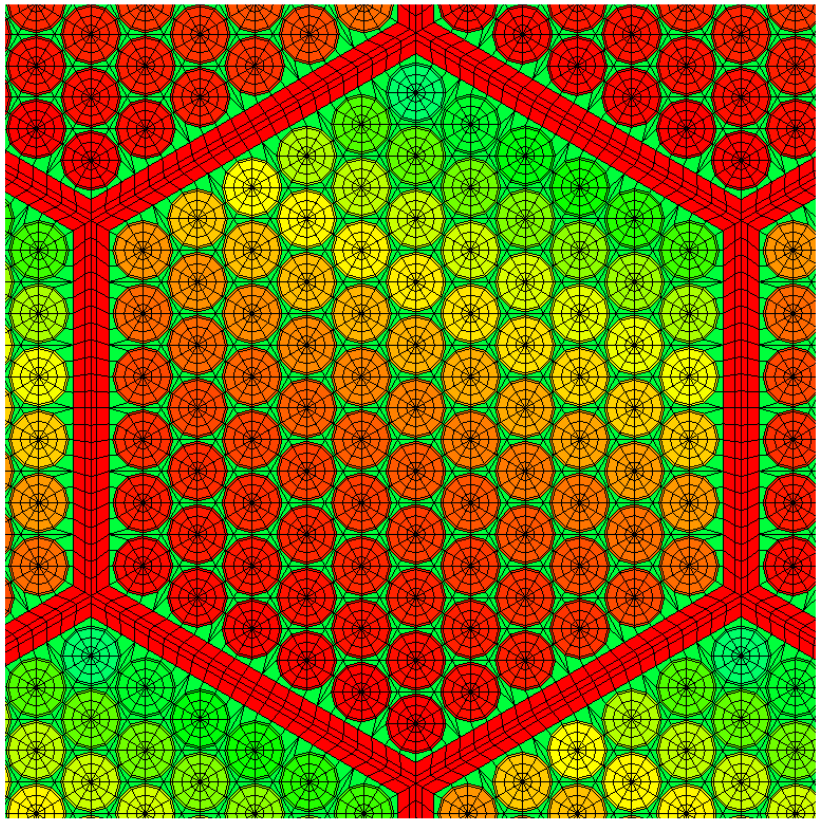

Figure 5.2.1.4 Seven-heterogeneous assembly AFR-100 mesh showing details of focal assembly and surrounding assembly.

\subsubsection{Multigroup Cross Section Generation Procedure}

PROTEUS-SN requires multigroup cross section data as input. For the SHARP zoom calculations, this data is processed with the $\mathrm{MC}^{2}-3$ code using a simple procedure for the homogeneous calculation, and a more complex procedure for the two heterogeneous calculations. 
For the homogeneous full core calculation, each axial region of each assembly was homogenized and simulated as independent compositions in a 1041-group $\mathrm{MC}^{2}-3$ infinite medium calculation. The full core geometry was approximated with a volume-equivalent R-Z geometry in TWODANT using the 1041-group cross sections to obtain the correct flux distribution across the core. The 1041group flux distribution is then used to collapse the cross sections to broad groups (9-group, 33group).

For the two calculations with heterogeneous fuel assembles (single-heterogeneous and sevenheterogeneous), a more complex procedure is employed to consider heterogeneous effects. The homogeneous procedure in the preceding paragraph is first used to obtain 1041-group cross sections and fluxes in the entire core for homogenized regions. Then, all fuel regions are re-processed using heterogeneous cylindrical geometry to approximate the rings in the assembly (ring 1 fuel, ring 1 clad, coolant, ring 2 clad, ring 2 fuel, ring 2 clad, ring 2 coolant, etc.). The appropriate 1041-group flux from TWODANT is used as an external spectrum source. The cross sections are then collapsed to broad groups for each fuel region, and re-merged with the homogeneous assembly cross sections from the first step. Homogeneous fuel cross sections are discarded. In both zooming calculations considered here, all of the heterogeneous assemblies are fuel assemblies. If the focal assembly (or neighboring assembly) was a control assembly, an extra control assembly calculation would be required.

\subsubsection{Code-to-Code Verification with Legacy Solver}

Code-to-code verification was performed for the homogeneous calculation using the DIF3DVARIANT transport code as a reference. Both the PROTEUS-SN and DIF3D-VARIANT calculations solved identical geometry using the same cross section data, however, DIF3D uses a different representation of chi (fission neutron energy spectrum) for compositions than PROTEUS. The eigenvalue and axially-integrated assembly powers showed excellent agreement as seen in Table 5.2.3.1 and Table 5.2.3.2. The $-181 \mathrm{pcm}$ difference in eigenvalue is due to the compositional chi treatment in DIF3D. The integrated assembly powers match with less than $2 \%$ difference, except for a few peripheral shield assemblies, which exhibit differences up to $10 \%$. The shield errors originate in DIF3D where the leakage is not well-modeled and thus PROTEUS more accurately calculates the leakage effects and is the more accurate result. To summarize, the PROTEUS-SN homogeneous model yielded results consistent with DIF3D-VARIANT, to the extent to which DIF3D is known to be accurate.

Figure 5.2.3.1 is a visual comparison of the power distribution computed by DIF3D-VARIANT (left column) and PROTEUS-SN (right column) at three axial heights: the active core outlet (top), midpoint (middle), and inlet (bottom). The same color scale is used in all figures. The DIF3DVARIANT calculations use 1/3rd core symmetry; a dashed box is placed around the comparable geometry in the PROTEUS-SN figures. 
Table 5.2.3.1 K-effective comparison for homogeneous assembly calculation.

\begin{tabular}{|l|c|}
\hline Code & K-Effective \\
\hline DIF3D-VARIANT (33g, P3P1) & 1.01816 \\
\hline PROTEUS-SN (33g, L3T5, P1) & 1.01635 \\
\hline Delta K (pcm) & -181 \\
\hline
\end{tabular}

Table 5.2.3.2 Integrated power comparison for homogeneous assembly calculation.

\begin{tabular}{|l|c|}
\hline Assembly Type & $\begin{array}{c}\text { Max Rel. Diff. in Assembly } \\
\text { Power (9g) }\end{array}$ \\
\hline Control & $1.9 \%$ \\
\hline Driver & $0.2 \%$ \\
\hline Reflector & $1.4 \%$ \\
\hline Shield & $10.2 \% *$ \\
\hline
\end{tabular}



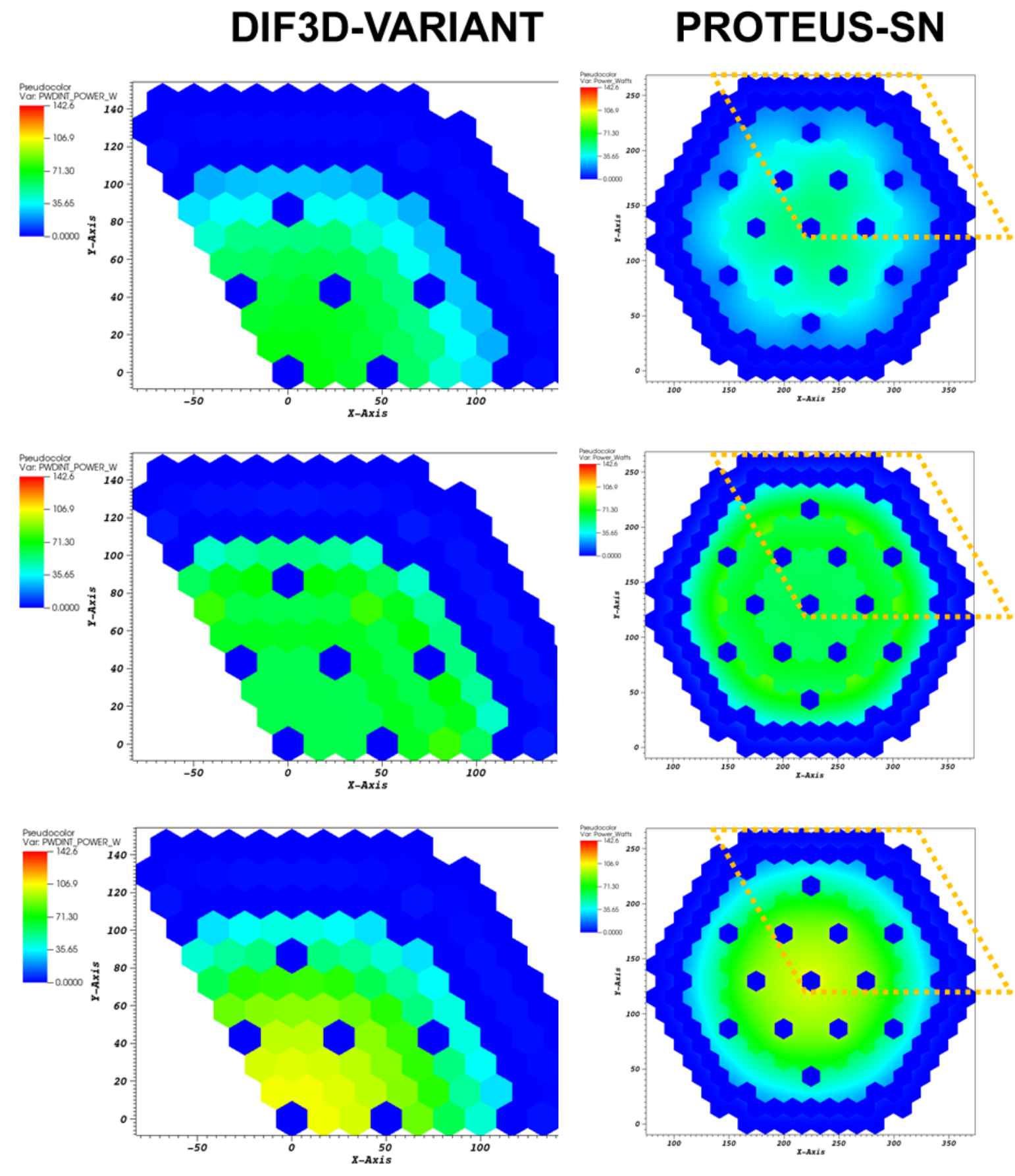

Figure 5.2.3.1 DIF3D vs. PROTEUS: Power distribution (W/cc) in axial slices of active core: (top) outlet at $\mathrm{z}=216 \mathrm{~cm}$, (middle) center at $\mathrm{z}=156 \mathrm{~cm}$, (bottom) and inlet at $\mathrm{z}=97 \mathrm{~cm}$. 


\subsubsection{Neutronics Results}

The homogenous, single-heterogeneous, and seven-heterogeneous assembly models were simulated with PROTEUS-SN. The eigenvalue is consistent (within $10 \mathrm{pcm}$ ) between all three cases as shown in Table 5.2.4.1. The computational requirements of each simulation are also shown. The computational time quickly increases as the mesh size increases. The trend is not linear because the actual complexity of the problem increases with increasing fidelity (heterogeneous materials as well as additional mesh elements).

The axial power distribution (integrated radially within the focal assembly) was consistent between all three simulations. The total power in the focal assembly was also consistent between all three simulations (within $0.5 \%$ ). Therefore, we conclude that the integral parameters in the focal assembly are not sensitive to homogenization or conversely de-homogenization of itself nor the surrounding assemblies, provided the multigroup cross sections include heterogeneous geometry effects as explained in Section 5.2.2.

Table 5.2.4.1 K-effective comparison for all neutronics zooming calculations.

\begin{tabular}{|c|c|c|}
\hline $\begin{array}{c}\text { PROTEUS-SN } \\
\text { Mesh } \\
(33 g, \text { L3T5, P1) }\end{array}$ & K-Effective & $\begin{array}{c}\text { Solve Time } \\
\text { (Nodes, Wall Clock Time) }\end{array}$ \\
\hline Homogeneous & 1.01635 & $\begin{array}{c}802 \text { core-hours } \\
\text { (256 BG/Q nodes, } 11 \mathrm{~min}) \\
\end{array}$ \\
\hline $1-\mathrm{Het}$ & 1.01634 & $\begin{array}{c}\text { 2,280 core-hours } \\
\text { (256 BG/Q nodes, } 33 \mathrm{~min}) \\
\end{array}$ \\
\hline 7-Het & 1.01626 & $\begin{array}{c}11,965 \text { core-hours } \\
\text { (1024 BG/Q nodes, } 43 \mathrm{~min})\end{array}$ \\
\hline
\end{tabular}




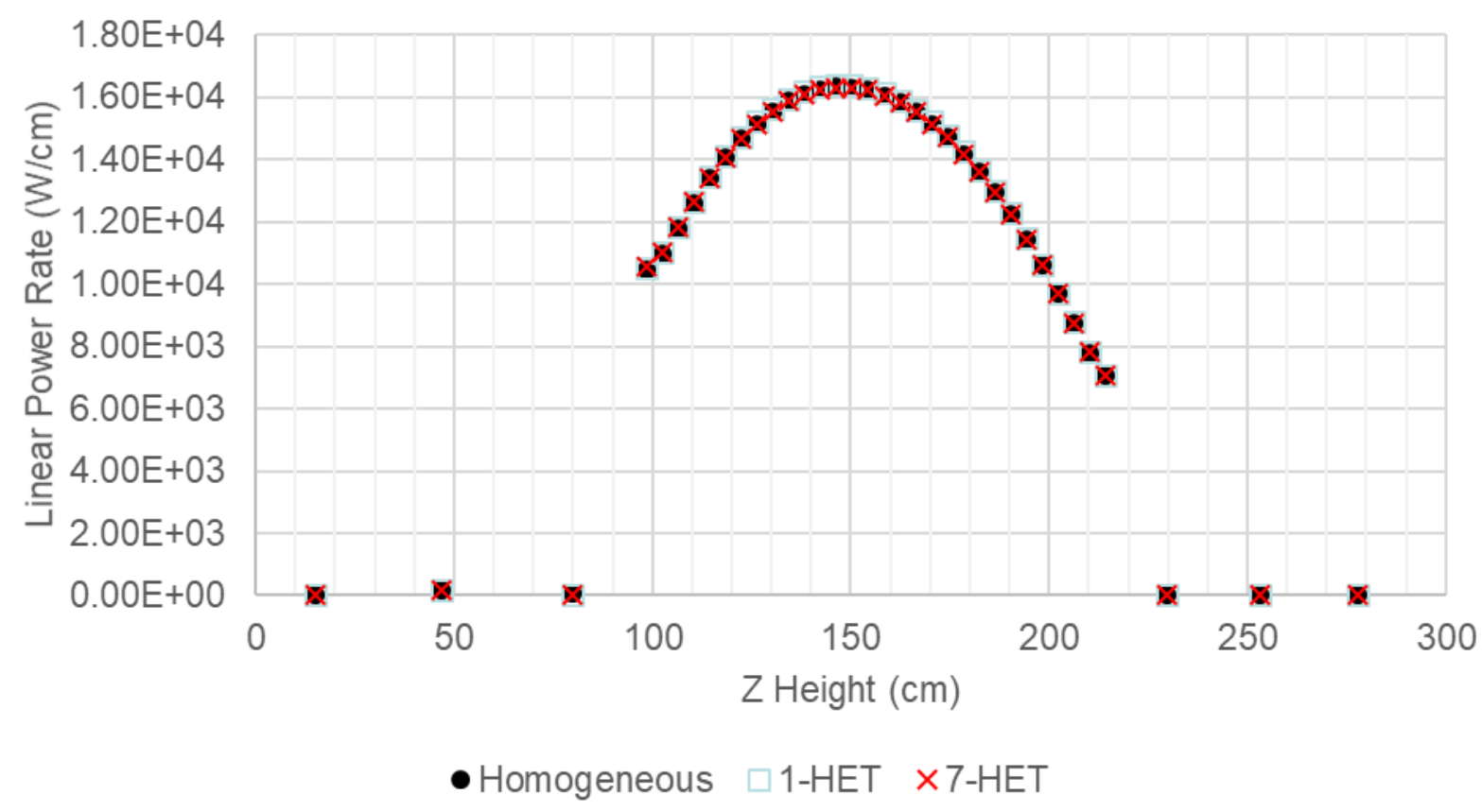

Figure 5.2.4.1 Radially-integrated axial power distribution $(W / \mathrm{cm})$ in focal assembly for various mesh models.

Figure 5.2.4.2 is a visual comparison of the power distribution computed by PROTEUS-SN for all three meshes: homogeneous (left), single-heterogeneous (middle) and seven-heterogeneous (right) at three axial heights: the active core outlet (top), midpoint (middle), and inlet (bottom). The same color scale is used in all figures. As the focal assembly and neighboring assemblies are successively de-homogenized, the power tilt in these assemblies comes into focus. While the total assembly integrated powers are nearly identical in all three mesh cases, the peak power intensity (W/cc) is larger when the pins are represented explicitly, as shown with the red color in the two right columns. The power in the homogeneous assembly is smeared across a larger volume and therefore appears lower. It is noted that no attempts were made to compared the pin power distribution in the focal assembly with the result obtained from the legacy calculations because the implemented pin power reconstruction method in the legacy code (i.e., RCT) is inconsistent with the neutron flux solver (i.e., DIF3D/VARIANT) used in the AFR-100 design. The RCT code reconstructs the pin power distributions by interpolating neutron fluxes obtained from a finite difference diffusion equation solver for six triangular nodes per a single hexagonal node, while the variational transport equation solver (i.e., DIF3D/VARIANT) was used for the AFR-100 design: i.e.., the comparison of high-fidelity solutions with the resulting power distributions from low order finite difference diffusion equation may mislead. 
Hom
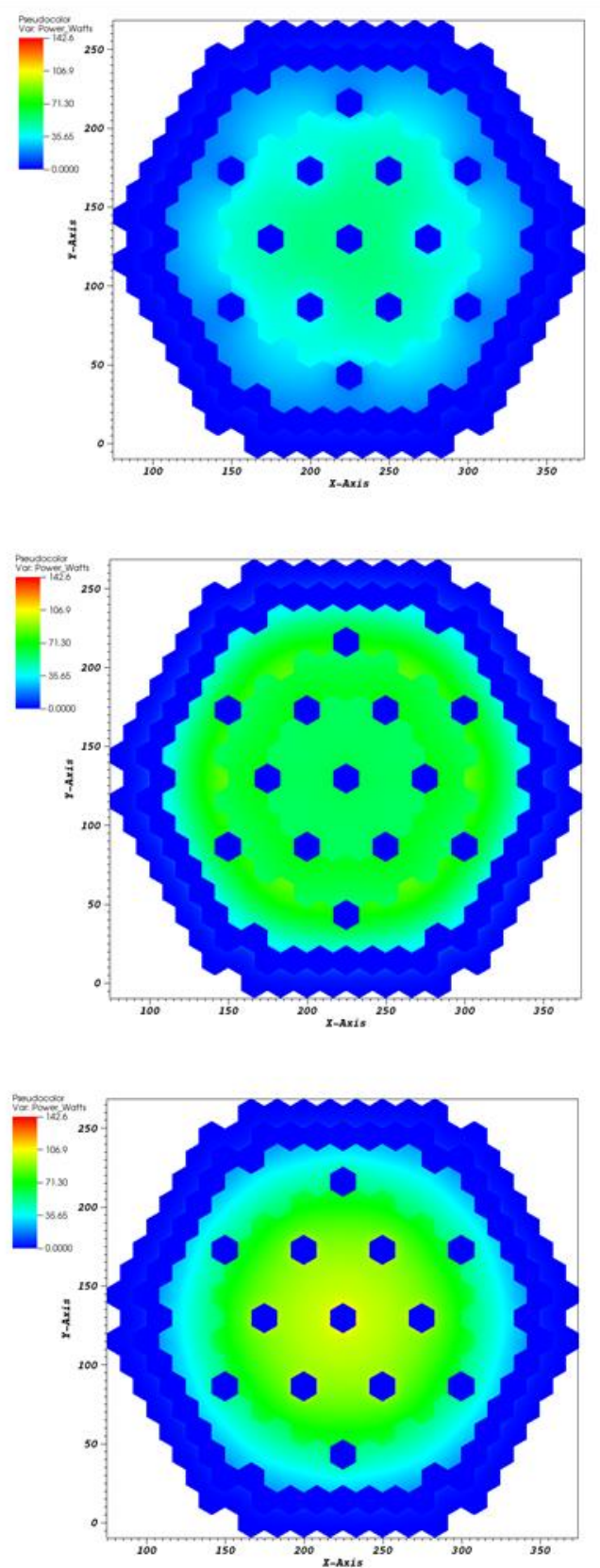

\section{1-Het}
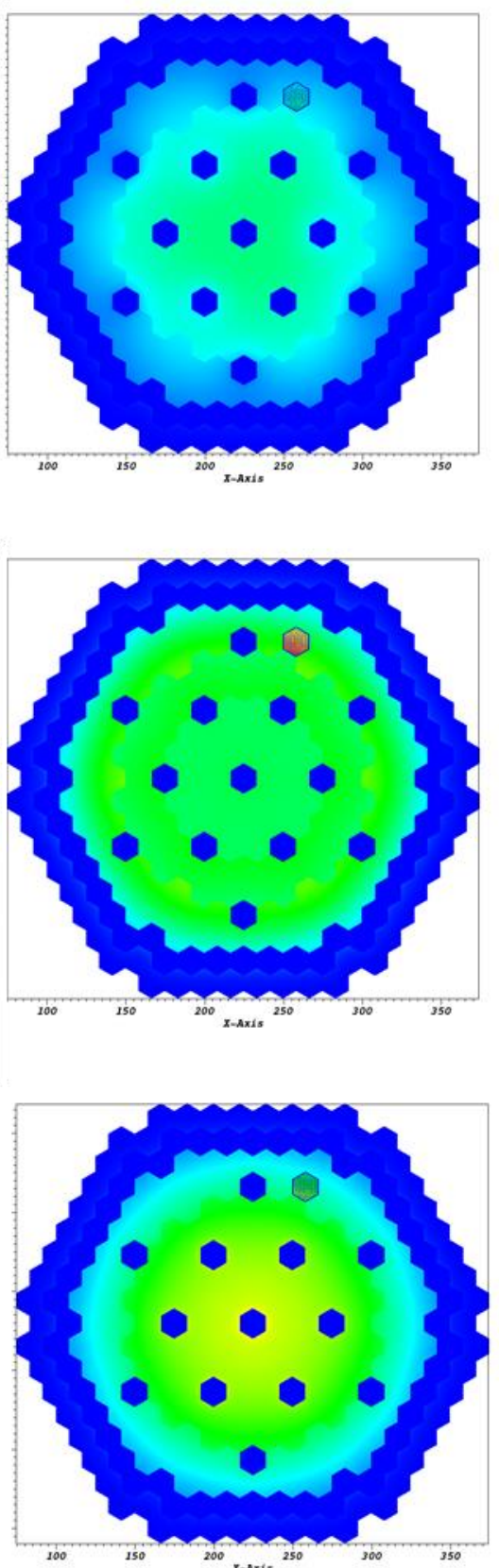

7-Het
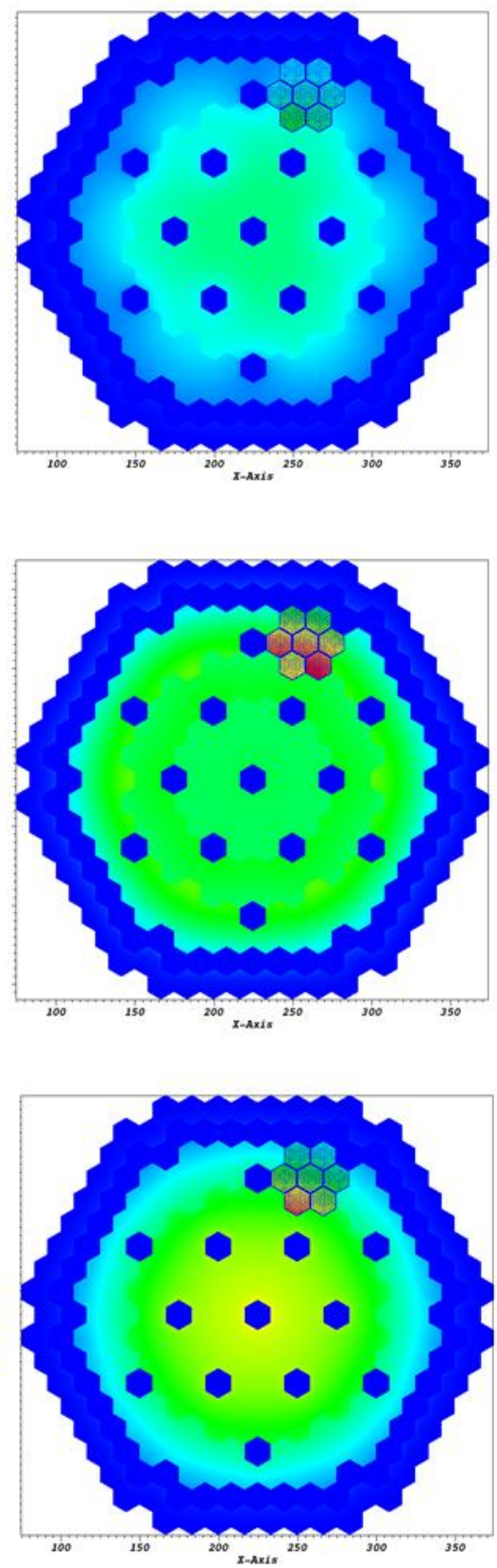

Figure 5.2.4.2 Power distribution (W/cc) in axial slices of active core: (top) outlet at $\mathrm{z}=216$ $\mathrm{cm}$, (middle) center at $\mathrm{z}=156 \mathrm{~cm}$, (bottom) and inlet at $\mathrm{z}=97 \mathrm{~cm}$.

Next, the focal assembly pin powers computed with the two zooming calculations were compared in Figure 5.2.4.3. Each pin power matched within $0.4 \%$. The radial power tilt is seen in 
the coloration of the figure on the right, and in the oscillation of power as the pins are identified and plotted in each successive ring. Note that the pin numbering here runs clockwise within a ring instead of the standard counterclockwise. The axial power distribution of the center pin as well as outer corner pins 67 (higher power) and 82 (lower power) were compared as well in Figure 5.2.4.4. These power distributions showed excellent agreement as well. Both of these plots support the conclusion that the single-heterogeneous model is adequate and acceptable for generating detailed, pin-by-pin axial power distributions in an SFR fuel assembly.
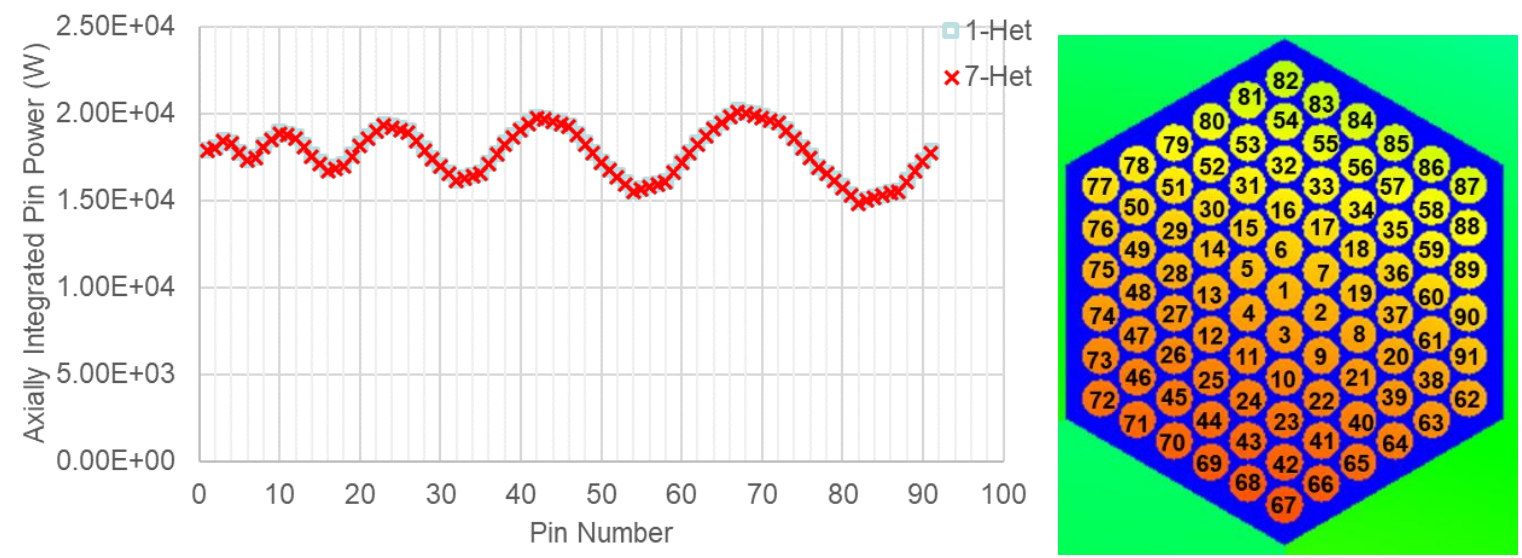

Figure 5.2.4.3 (left) Pin power $(W)$ in each pin of focal assembly; (right) Pin numbering scheme.

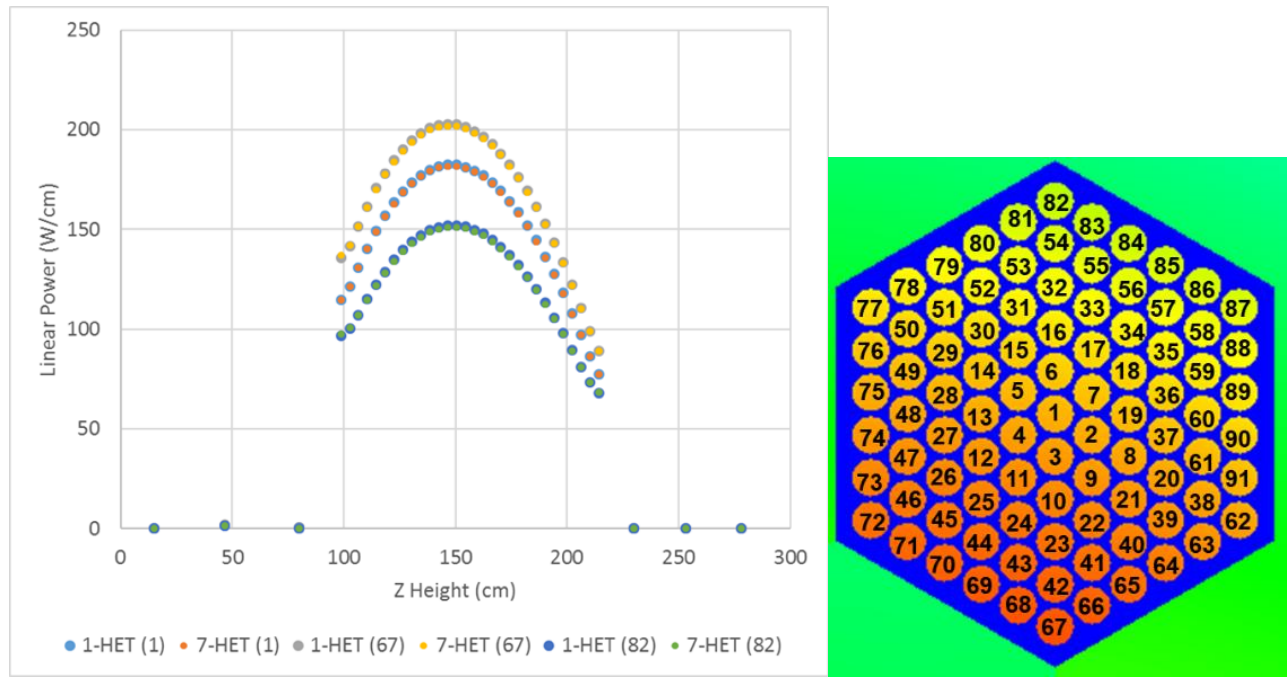

Figure 5.2.4.4 (Left) Linear power (W/cm) in selected pins of focal assembly; (right) Pin numbering scheme.

Finally, the normalized axial power distribution for all pins in the focal assembly was plotted on the same chart in Figure 5.2.4.5. Despite the power tilt in this assembly, all 91 pins in the assembly have nearly identical normalized power distributions. This significantly facilitates the data transfer 
to Nek5000, since a single axial shape can be defined and multiplied by individual pin powers to scale it.

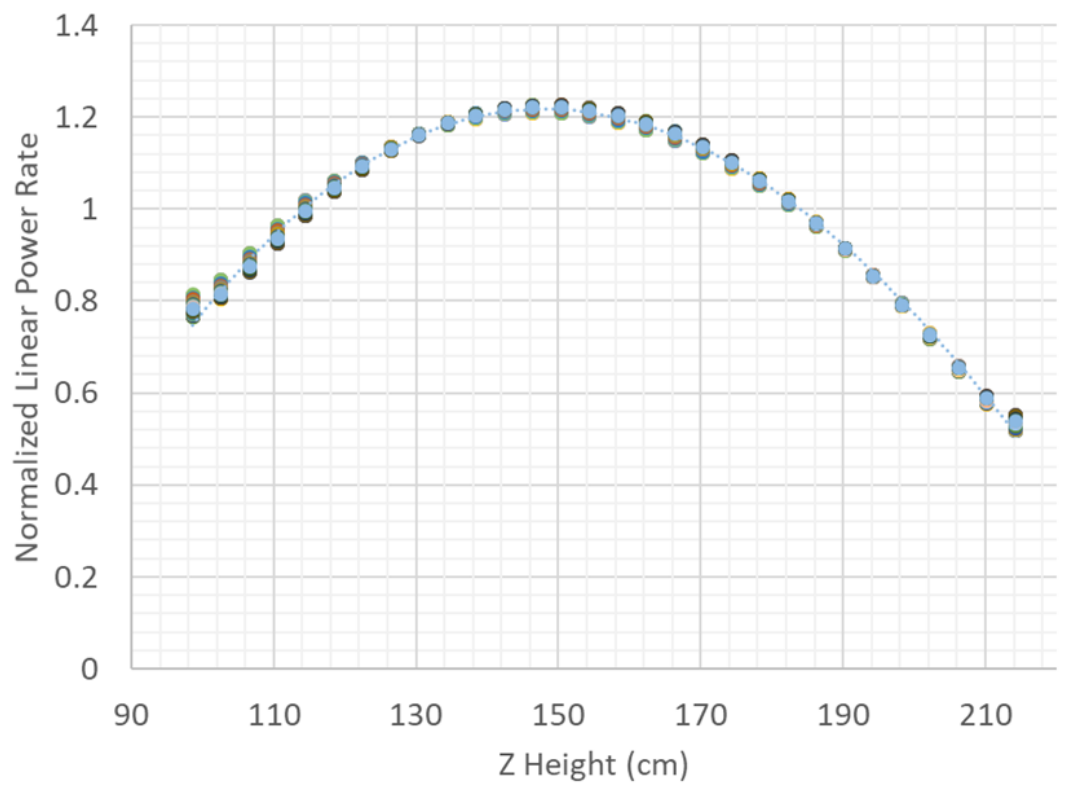

Figure 5.2.4.5 Normalized linear power in all pins of focal assembly.

\subsubsection{Neutronics Zooming Summary}

Three neutronics calculations of the AFR-100 core were performed to demonstrate the SHARP zooming concept. First, a conventional homogeneous assembly calculation was performed as a baseline and for comparison with legacy solvers. Progressively more heterogeneity was introduced in two subsequent calculations: the single-heterogeneous assembly calculation modeled a single focal assembly with pin-by-pin level detail, and the seven-heterogeneous assembly calculation modeled the focal assembly and the surrounding ring of six fuel assemblies as pin-by-pin. The power distribution in the focal assembly was compared across the three simulations.

To summarize, the computed integral quantities (eigenvalue, total assembly power, axial power distribution) were extremely consistent across all three simulations. The individual pin powers were nearly identical between the two heterogeneous simulations. At this time, the pin powers were not constructed for the homogeneous simulation. The current findings support the recommendation to use a single-heterogeneous model in neutronics provided the focal assembly is a fuel type and surrounded by other similar assemblies. If the focal assembly or neighboring assemblies contain control material (strong absorber), then the sensitivity study should be repeated to verify whether a single pin-by-pin assembly representation is sufficient. It is possible that reconstruction of pin powers from a homogeneous simulation may also be sufficient, but this has not yet been investigated. 


\subsection{Thermal Hydraulics Calculations}

Nek5000 was selected as the thermal hydraulic solver for the zooming. The power rate of each pin is calculated in the preceding PROTEUS neutronics calculations. This section describes the details of the thermal hydraulic calculations.

\subsubsection{Thermal Hydraulic Model}

A heterogeneous seven-assembly model (91-pin bare bundles including the focal assembly and its neighboring 6 assemblies) was built for Nek5000. Fuel, cladding, duct wall and the sodium gap between the assemblies are taken as solid and modeled for conjugate heat transfer. The mesh and assembly numbering is shown in Figure 5.3.1.1, where the focal assembly is labeled Assembly 1. Adiabatic boundary conditions are applied to the outermost boundary of the model. The rest of the core is not modeled, both because it is not needed, and because modeling it as homogeneous porous medium would contribute errors, as explained below. Figure 5.3.1.2 shows the rod temperatures in the 7-assembly and single assembly models. The nearly identical results indicate that the influence from the neighboring assemblies is very small. Heat conduction in the assembly duct and interassembly sodium gap homogenizes the temperature difference circumferentially and reduces the impact of neighboring assemblies. Moreover, a previous investigation [Yu 2015] has shown that a homogeneous porous medium model can over predict the duct wall temperature by $80 \mathrm{~K}$. Given that taking into account the full core with a porous medium model not only increases the complexity of the model but also introduces large deviations on the boundary, the 7-assembly model is suitable for SHARP zooming calculations.

Three models are adopted in the neutronics calculation: the homogeneous model, singleheterogeneous model and seven-heterogeneous model. The power rate for each pin calculated by these three models are imported into Nek5000 as the energy source term.

Assemblies modeled homogeneously in neutronics yield uniform pin powers and shapes across the assembly to Nek5000. While in reality, the neutronics solution on a homogeneous assembly has a gradient, there is no easy way to reconstruct pin powers consistent with this gradient for an offline transfer to a thermal hydraulics calculation. Therefore, the average pin power is calculated and applied to all pins. This is clearly a shortcoming of attempting to couple high fidelity $\mathrm{T} / \mathrm{H}$ calculations with low fidelity neutronics calculations. On the other hand, assemblies modeled heterogeneously in neutronics yield unique powers and shapes in each pin.

The total assembly power in the focal assembly and surrounding six assemblies predicted by the three neutronics model is consistent (Figure 5.3.1.3). The average velocity and numbering scheme for the 7-assembly model is shown in Figure 5.3.1.4. Assembly 1 is the focal assembly which is an outer fuel assembly. All surrounding assemblies are also outer fuel assemblies, except for Assembly 4 , which is a middle core assembly having a unique axial power distribution similar to the inner fuel assembly discussed in Chapter 4. Assembly 5 has the highest total power. 


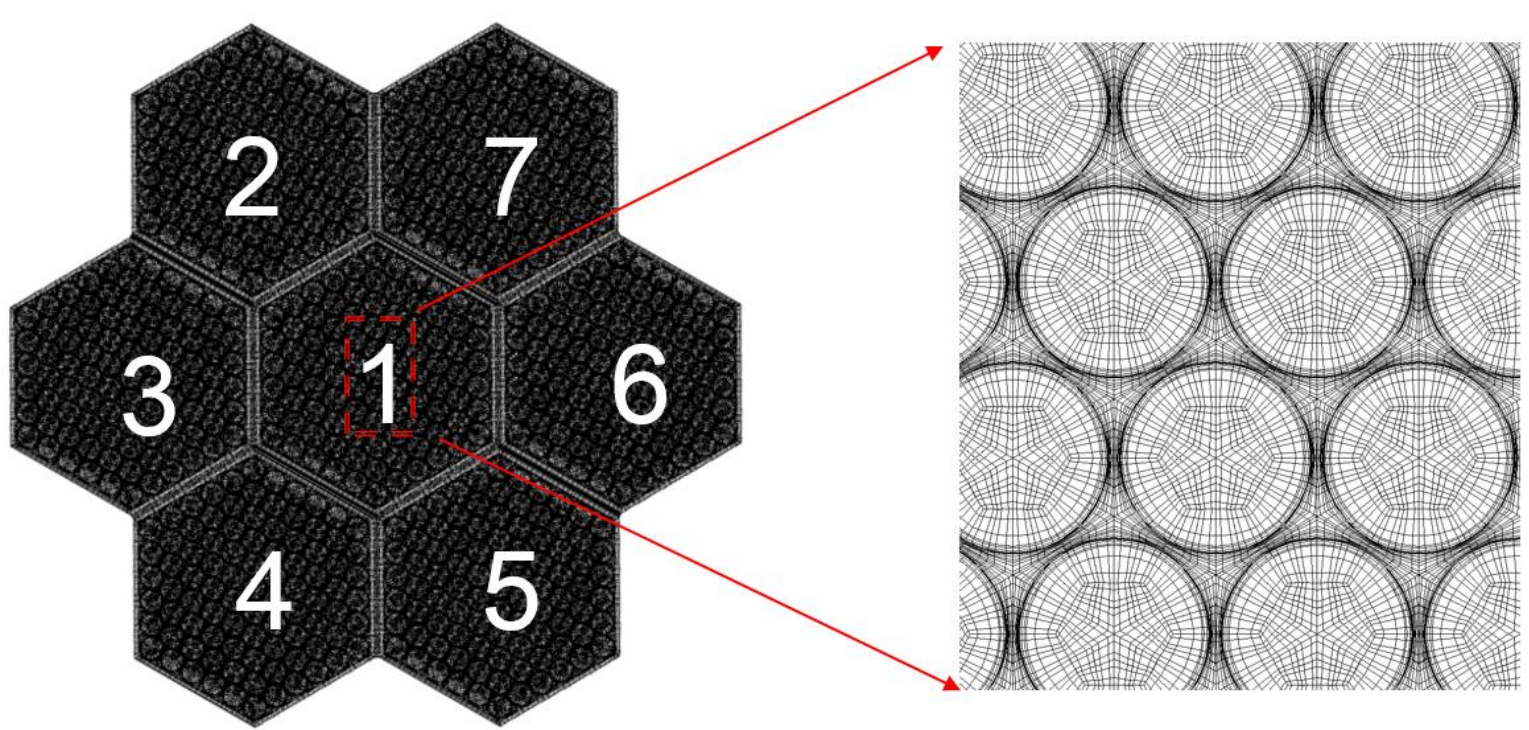

Figure 5.3.1.1 The mesh of 7-assembly model

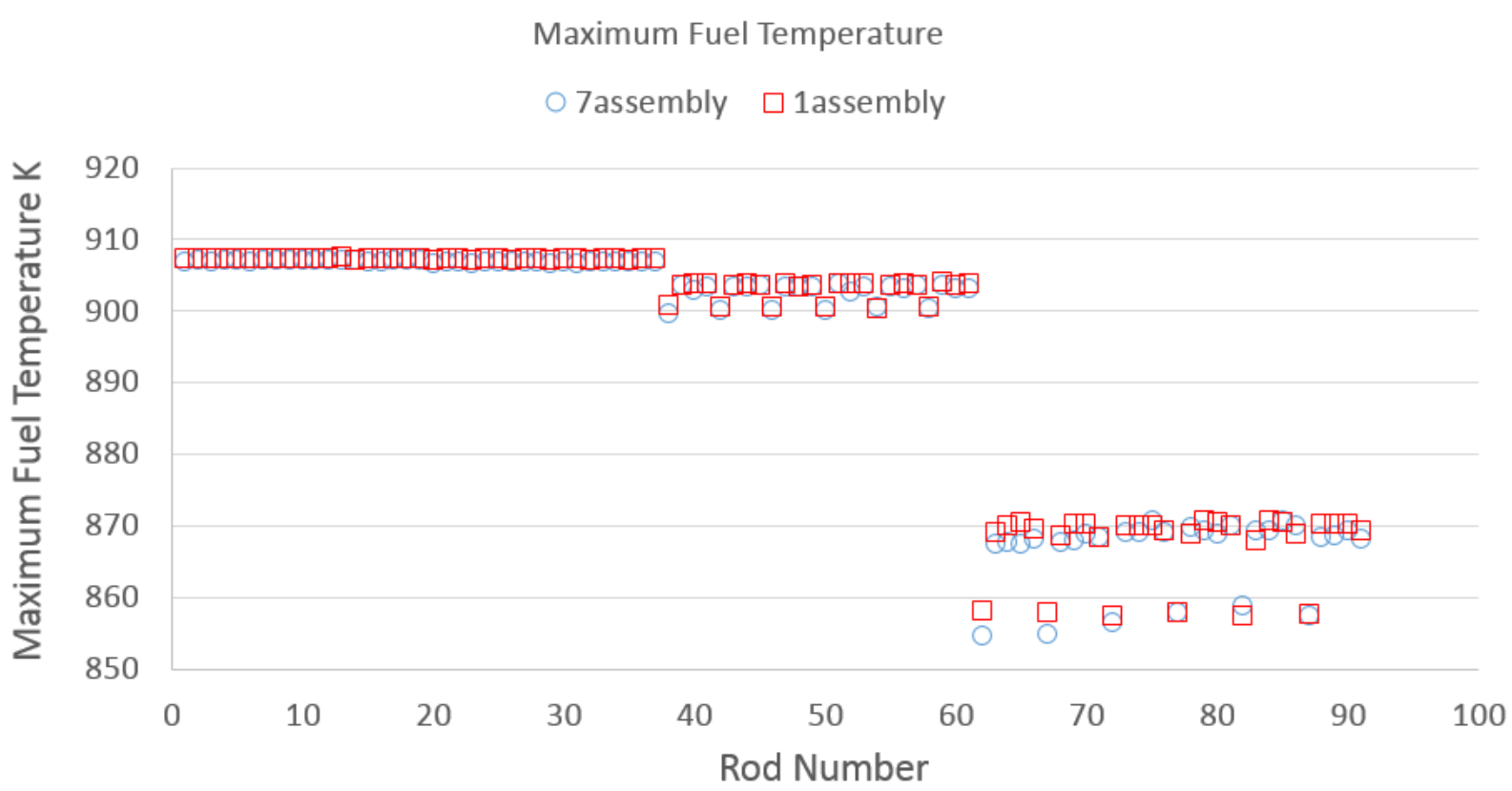

Figure 5.3.1.2 Maximum fuel temperature of 7-assembly model and single assembly model 


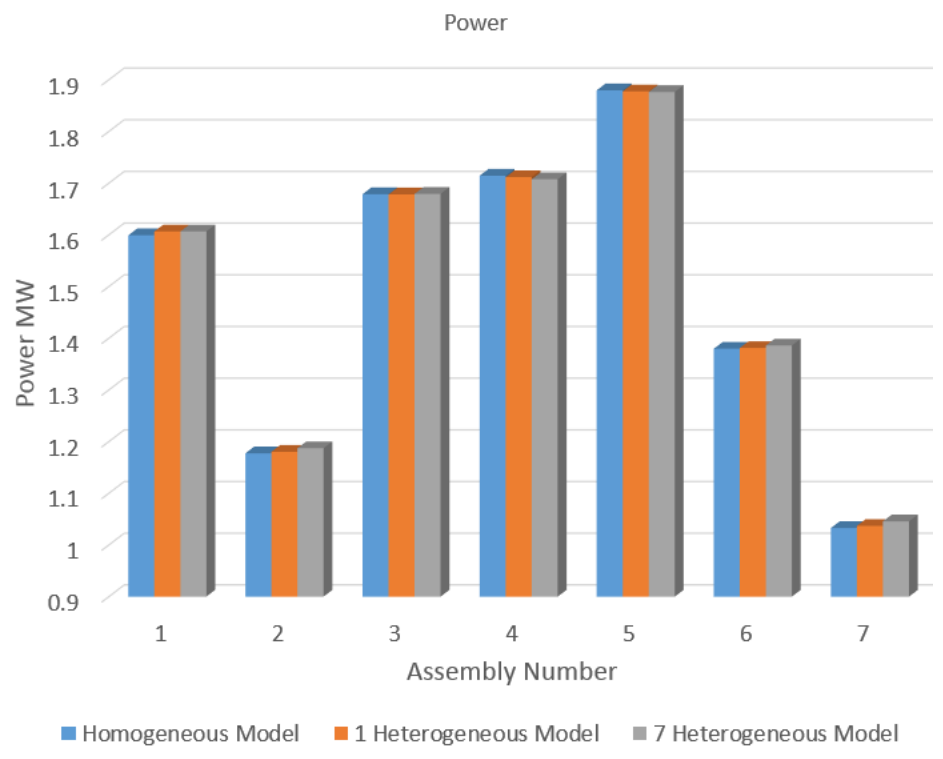

Figure 5.3.1.3 Total power for each assembly from three models
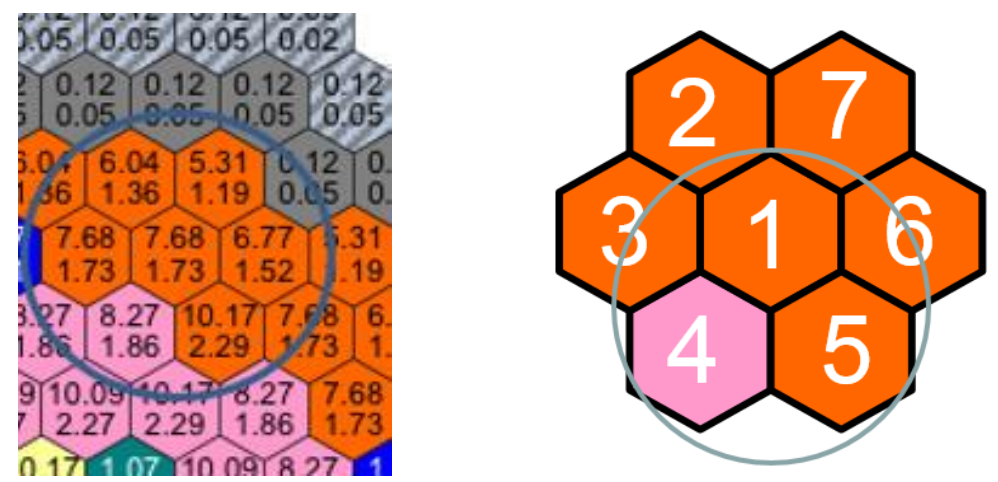

Figure 5.3.1.4 The numbering scheme and velocity distribution for 7-assembly model

\subsubsection{Thermal Hydraulic Results}

The temperature distribution of the focal assembly using powers derived from the homogeneous and heterogeneous neutronics models are shown in Figure 5.3.2.1. We note again that the neutronics solution on a homogeneous assembly has some gradient but there is no easy way to reconstruct the pin-by-pin powers consistent with this gradient for transfer to a pin-by-pin thermal hydraulics calculation. The homogeneous model therefore predicts the same temperature distribution as that of an isolated single assembly. The neighboring assemblies have little effect on the temperature distribution of focal assembly. The obvious and correct temperature gradient is observed in the heterogeneous model. The temperature gradient occurs because the assemblies and pins closer to the center of the core have higher power rates. 


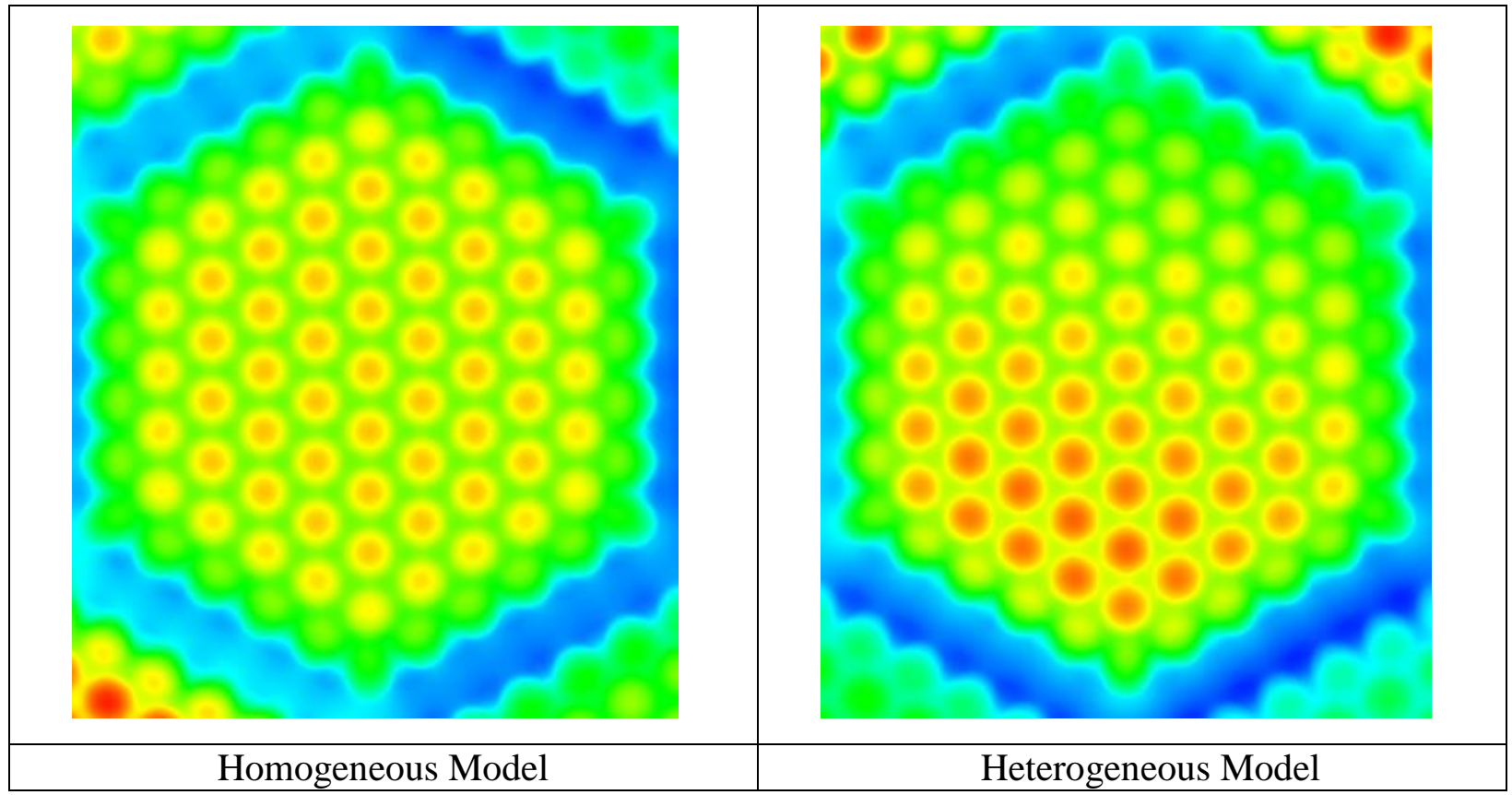

Figure 5.3.2.1 Temperature distribution at active core outlet of focal assembly with homogeneous model heterogeneous neutronics models

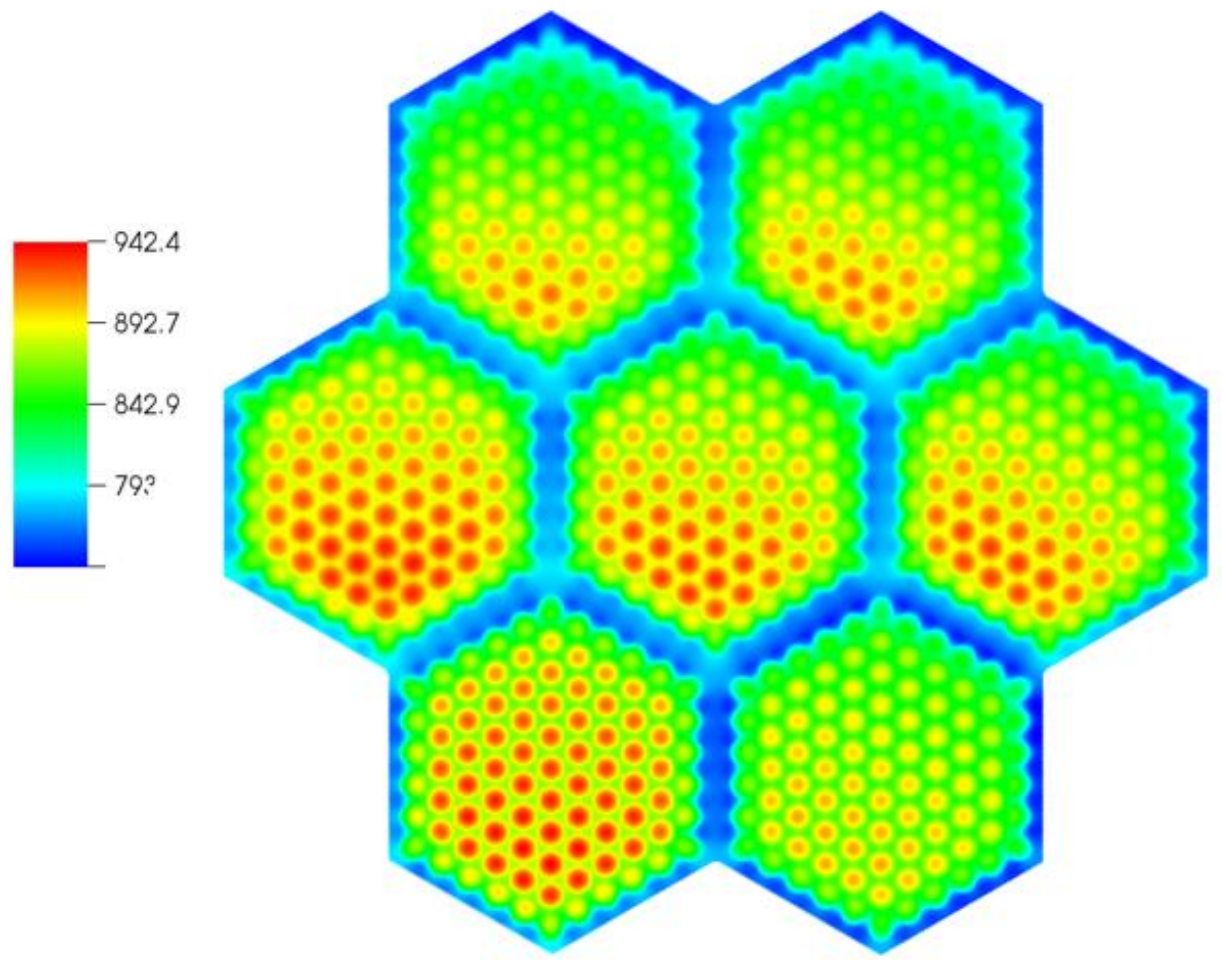

Figure 5.3.2.2 Temperature distribution at active core outlet of 7 assemblies using heterogeneous neutronics model 
The Nek5000 7-assembly model using powers from the 7-heterogeneous assembly neutronics model yielded the temperature distribution shown in Figure 5.3.2.2. The temperature tilt, or temperature bias, exists in all assemblies. Due to this temperature bias, the hot spot of each assembly no longer occurs in the center of the assembly. In other words, since the sevenheterogeneous model can reproduce the detailed temperature distribution in all the assemblies, it can predict the location of the hot spot for each assembly precisely. For instance, the hot spot of assembly 4 (middle core assembly) appears at the outlet of the active core due to its unique power distribution. The hot spot of the focal assembly occurs below the active core outlet. From the results, we can predict that the maximum fuel temperature (958.5 C) appears in Assembly 4. Although Assembly 5 has maximum total power, the larger average flow velocity through this assembly (assigned as part of the specification) decreases the maximum fuel temperature substantially. The SHARP zooming capability therefore provides a powerful tool to predict the location and temperature of hot spots in the focal assemblies in confirmatory design calculations.

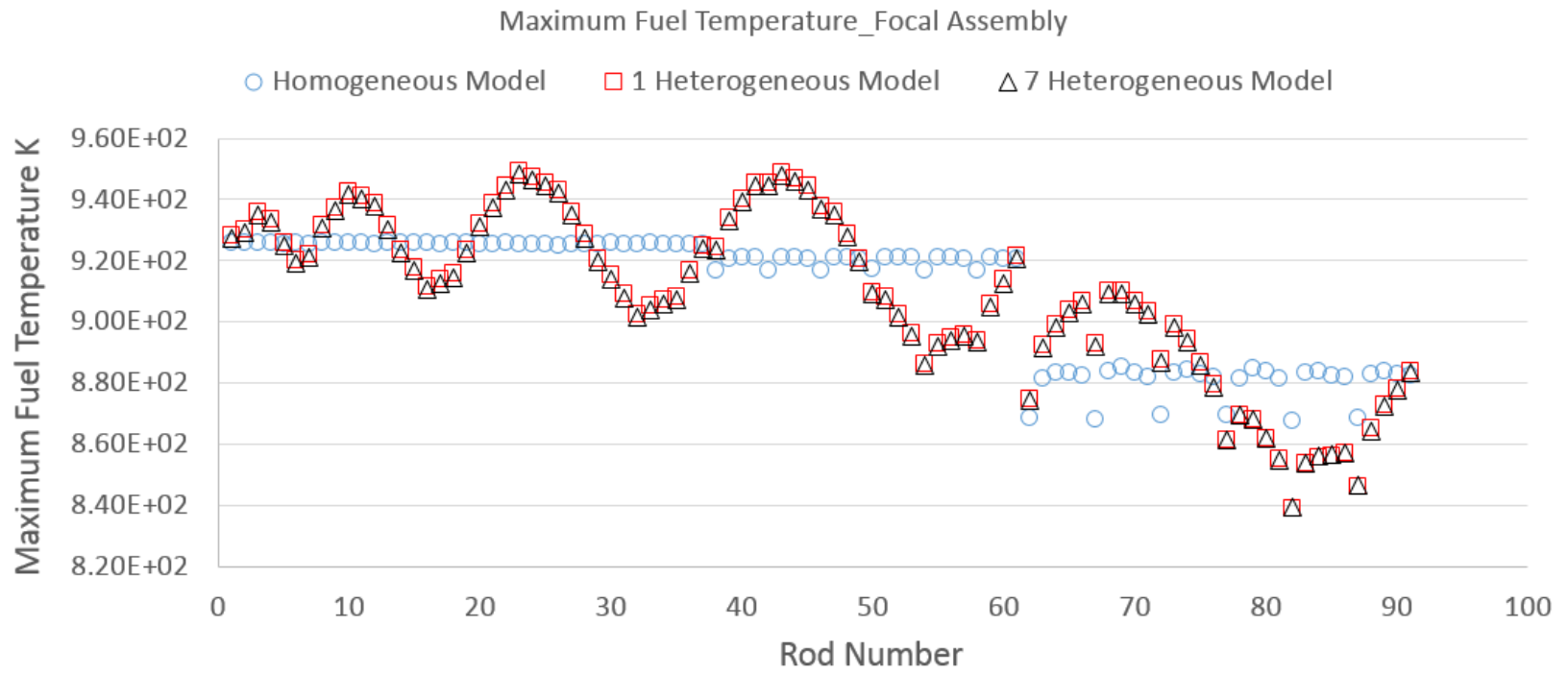




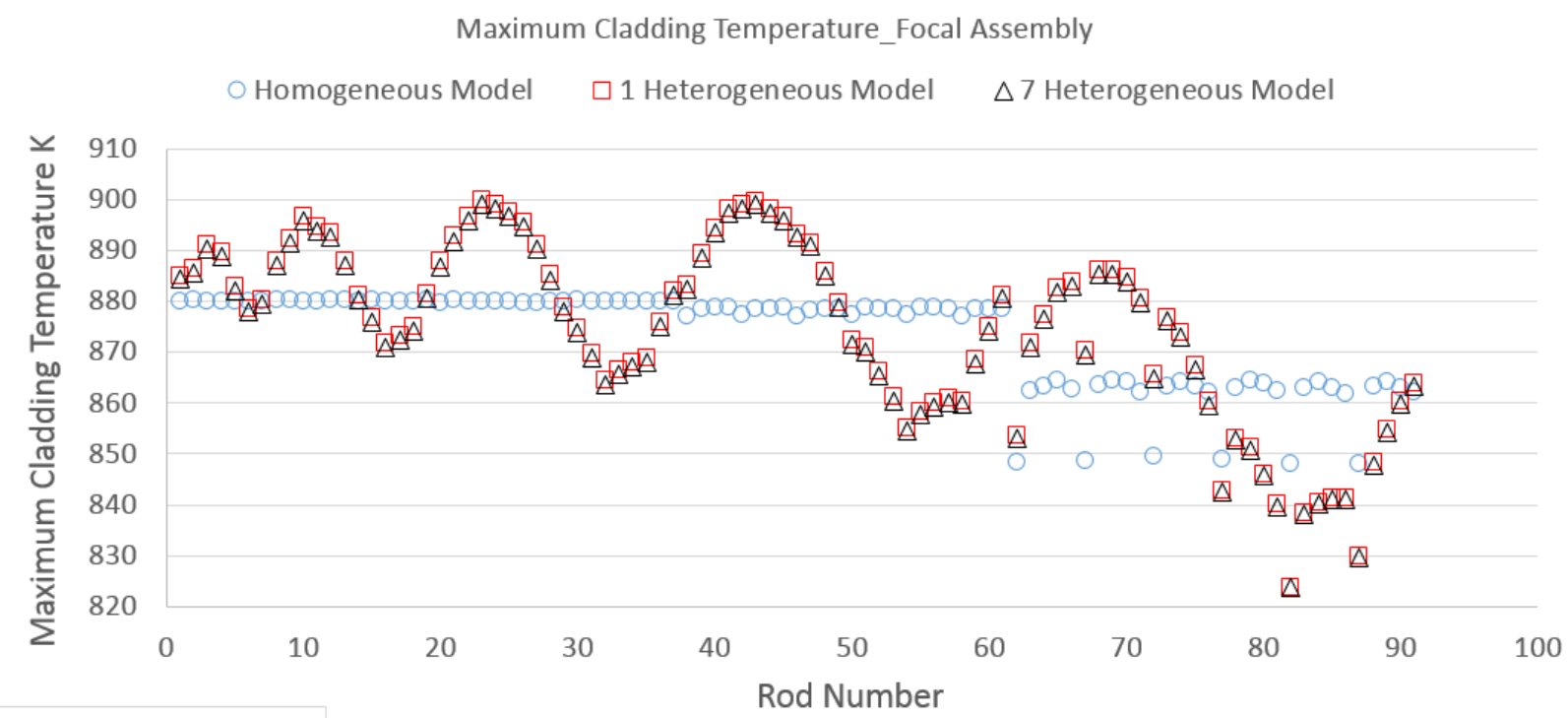

Figure 5.3.2.3 Cladding and fuel temperature distributions in focal assembly

The detailed maximum cladding and fuel temperature in focal assembly presented in Figure 5.3.2.3 further confirm the primary conclusion mentioned above. The maximum cladding and fuel temperatures from the single-heterogeneous and seven-heterogeneous neutronics model are identical. Individual SFR assemblies are confirmed to be insulated enough from each other from a thermal hydraulic perspective that as a result, only the focal assembly is required for heterogeneous modeling in the Nek5000 calculation. The homogeneous neutronics model predicts a uniform temperature in the center region which is more like a hot zone while the heterogeneous model can predict the specific location of the hot spot, near pin number 43 (Figure 5.3.2.4). 
Hot Zone Prediction from Homogeneous Model Heterogeneous Model

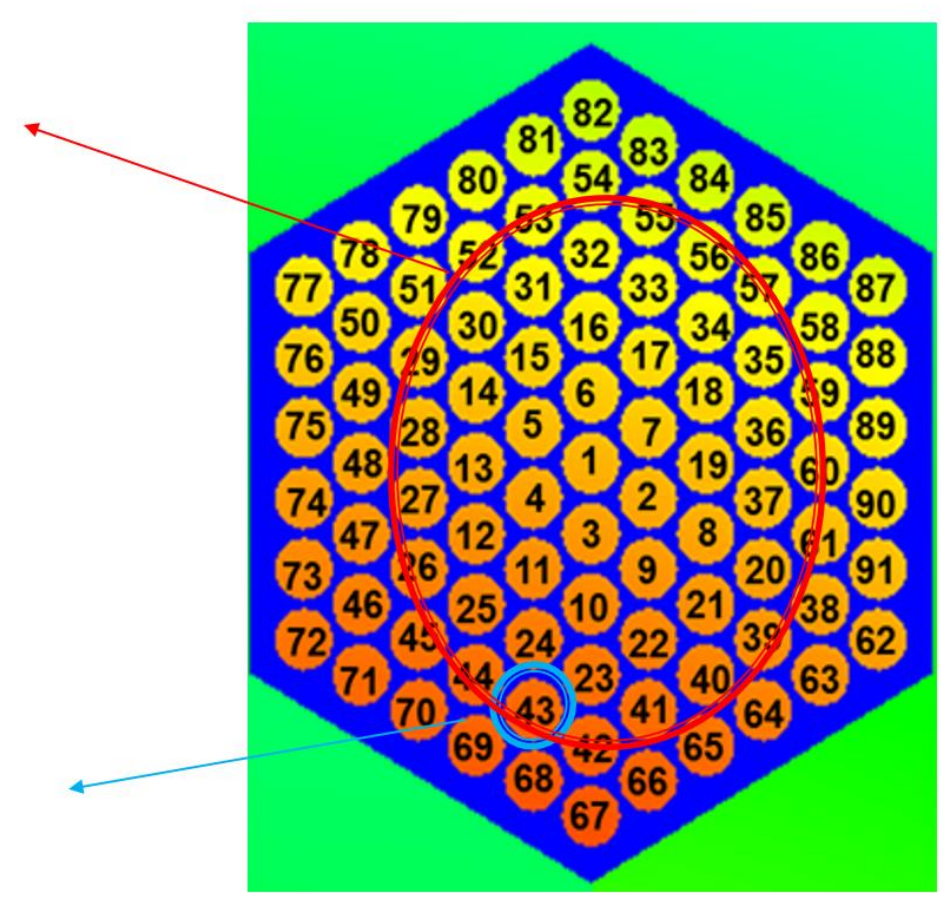

Figure 5.3.2.4 Hot zone prediction with homogeneous mode and hot spot prediction with heterogeneous model 


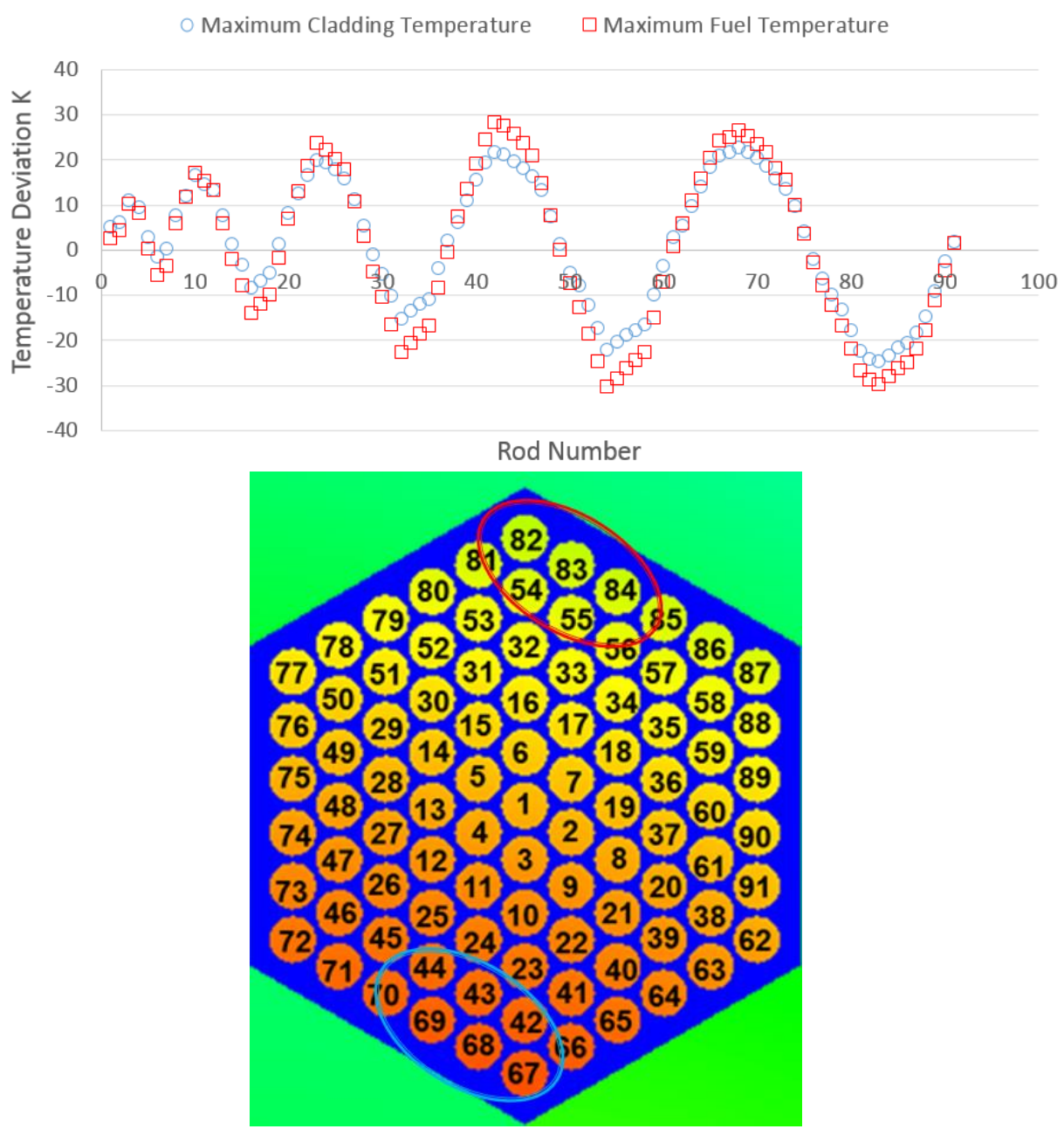

Figure 5.3.2.5 Absolute cladding and fuel temperature deviation in focal assembly between homogeneous model and heterogeneous model (top) and pin numbering (bottom)

The heterogeneous neutronics model can capture the temperature bias in each assembly while homogeneous model cannot. Figure 5.3.2.5 indicates that the homogeneous model can overestimate or underestimate the fuel and cladding temperature by about $30 \mathrm{C}$ (unless somehow the pin powers can be reconstructed - no capability exists for this work yet). For the case of a peripheral fuel assembly, it can underestimate the fuel temperate on one side of the assembly and overestimate it on the other side.

\subsubsection{Thermal Hydraulic Zooming Summary}

The pin-by-pin power distributions calculated by three PROTEUS neutronics full core models (homogeneous model, single-heterogeneous model and seven-heterogeneous model) are loaded into Nek5000 thermal hydraulic simulations of a limited part of the core in order to demonstrate the SHARP zoom concept. The following conclusions were derived: 
- Individual SFR assemblies are insulated enough from each other such that only the heterogeneous single assembly model for the focal assembly is sufficient in the thermal hydraulic calculation with the Nek50000 code.

- Use of SHARP zooming capability can identify the local hot spot location and predict more accurate maximum cladding/fuel temperatures than a homogeneous model used by legacy tools without pin power reconstruction (the differences from the homogeneous model is up to $30 \mathrm{~K})$.

- The SHARP zooming calculation, performed by coupling PROTEUS neutronics and Nek5000 thermal hydraulic simulations with varying fidelity and geometry, can be applied to multiple locations of the core where hot spots are expected. The neutronics model can be built once provided each focal assembly is explicitly represented. The thermal hydraulics calculation for multiple assemblies can be performed in parallel on a smaller geometry domain, which improves the computational efficiency and applicability of the SHARP tools significantly. 


\section{Conclusions}

The Simulation-based High-efficiency Advanced Reactor Prototyping (SHARP) toolkit is under development by the Nuclear Energy Advanced Modeling and Simulation (NEAMS) Campaign of the U.S. Department of Energy, Office of Nuclear Energy. After releasing the SHARP toolkit in 2016, the main focus of the Reactor Product Line (RPL) of the NEAMS Campaign was shifted toward testing, validation and verification of SHARP component codes. To better understand and exploit the benefits of advanced modeling simulations, the NEAMS Campaign initiated the "Sodium-Cooled Fast Reactor (SFR) Challenge Problems" multi-year task.

The SFR challenge problems include the assessment of hot channel factors (HCFs) and the demonstration of a zooming capability on the 100 MWe Advanced Fast Reactor (AFR-100) using the SHARP toolkit. The primary objective of the first challenge problem is to reduce or/and eliminate HCFs using the high-fidelity SHARP toolkit and eventually improve the SFR economics. The HCF is a quantified uncertainty that is involved in nominal calculation of a design parameter. The second challenge problem is the demonstration of the SHARP zooming capability, which is a hybrid core modeling and simulation scheme enabled by combining coarse-and-homogeneous meshes and fine-and-heterogeneous meshes. If both challenge problems are resolved using this SHARP toolkit, the economic competitiveness of an SFR is significantly improved by saving computation costs and enhancement of safety margins.

Computational models, mesh capabilities, and coupling procedures were developed for multiphysics calculations using the neutronics (PROTEUS) and thermal-hydraulic (Nek5000) component codes of the SHARP toolkit in order to demonstrate the HCF calculation capability and zooming concept. In this study, a one-way offline coupling method was employed where the neutronics solver PROTEUS computes the power profile (according to an initial temperature distribution guess), and the thermal hydraulics solver Nek5000 evaluates the peak temperature using the neutronics power profile. Temperature feedback to neutronics code for updating the power profile was not considered in order to save computation time and resources. If the initial temperature profile used in the neutronics calculation is reasonably accurate, the one-way offline method is valid because the neutronics power profile has relatively weak dependence on small temperature variation.

Several hot channel factors for metal fueled SFRs were identified through literature review as potential candidates for reduction or elimination through high-fidelity simulations. The HCFs of the peak temperatures at cladding outer surface, cladding inner wall surface, and fuel centerline induced by cladding manufacturing tolerance and uncertainties on the material thermo-physical properties were evaluated. Several HCFs are not sensitive to using smaller pin bundles for $\mathrm{T} / \mathrm{H}$ modeling and therefore a smaller model could be used if computational resources are not sufficient, particularly for wire wrap calculations. Additionally, comparing HCFs derived from bare rod bundle and wire wrap rod bundle models indicate that most HCFs are not sensitive to wire wrap 
modeling. The prediction from bare rod bundle is more conservative than that from wire wrap rod bundle. The wire wrap calculation involves additional geometric complexity, significant computational expense, and it also requires the conjugate heat transfer in the fuel pin to be neglected due to the difficulty of meshing. Thus, some extra error is introduced in wire-wrap calculations due to the neglect of the circumferential heat conduction. In this study, we use as ideal model as possible to demonstrate the capability of the SHARP tools for HCF calculations. One important finding is that the cost of random sampling techniques for estimating HCFs, which are more natural to assess uncertainties stochastically, can be reduced substantially by making appropriate and justified simplifications as shown in this work. As expected, the resulting HCFs obtained from the highfidelity SHARP calculations are generally smaller than those developed for the earlier SFRs because the most uncertainties involved in the modeling and simulations were reduced or eliminated. Some HCFs such as cladding circumferential temperature variation and wire orientation which cannot be accessed in previous study have also been computed accurately.

Full core AFR-100 simulations were carried out to test the feasibility of the SHARP zooming capability. Several bounding design parameters (i.e., peak temperatures at cladding inner wall surface and fuel centerline) and integral and average parameters (i.e., core multiplication factor, and assembly-average power distribution, etc.) were evaluated. The conclusion of this work is that the neutronics profile in a focal assembly can be accurately represented using full core PROTEUS model consisting of homogenized assemblies except the heterogeneously-modeled focal assembly. The detailed pin powers can be communicated to a Nek5000 model of a single heterogeneous fuel assembly due to absence of cross flow between the ducted fuel assemblies. Modeling such small portions of the core explicitly yields significant computational savings over an explicit full core model. These results should be verified for different assembly types (e.g. control) as some of the findings could change. Additionally, the improvement over legacy tool procedures should be quantified.

In summary, the PROTEUS and Nek5000 high fidelity tools developed under NEAMS can deliver more precise power distribution and thermal hydraulic results than previous tools. Not only can the HCFs can be obtained but also the information around hot spot regions can be explicitly analyzed. The results obtained with the SHARP toolkit has already demonstrated the potential reduction in calculated HCFs of SFRs compared to legacy techniques. The SHARP zooming concept can be employed in multiple location of the core to detect the location and temperature of the possible hot spots in the core as part of a confirmatory analysis calculation.

Future work is planned to apply the $\mathrm{HCF}$ and zooming calculation capabilities demonstrated in this study to the Versatile Test Reactor (VTR) and other fast reactors with different coolant types (lead or gas). 


\section{References}

[Adams 1975] C. H. Adams, "Specifications for VARI3D—A Multidimensional Reactor Design Sensitivity Code,” FRA-TM-74, Argonne National Laboratory (1975).

[Adams 1987] C. H. Adams, "GAMSOR Newsletter \#1," Internal Memo, June 26, 1987.

[Alcouffe 1984] R. E. Alcouffe, F. W. Brinkley, D. R. Marr, and R. D. O'dell, 'User's Guide for TWODANT: A Code Package for Two-Dimensional, Diffusion-Accelerated Neutral Particle Transport," LA-10049-M, Los Alamos National Laboratory (1984).

[Beitel 1984] J. C. Beitel, "Status of and Revised Input for SE2-ANL," Internal Memo, January 30, 1985.

[Brockmeyer 2017] Landon Brockmeyer, Lane B. Carasik, Elia Merzari, Yassin Hassan, Numerical simulations for determination of minimum representative bundle size in wire wrapped tube bundles, Nuclear Engineering and Design, Volume 322, 2017, Pages 577 590.

[Carelli 1980] M. D. Carelli, A. J. Friedland, "Hot Channel Factors for Rod Temperature Calculations in LMFBR Assemblies," Nuclear Engineering and Design 62 (1980).

[CD-Adapco 2009] "STAR-CCM+ User Guide", Published by CD-Adapco, 2009.

[Cheon 2017] J. S. Cheon, private communications for the fuel fabrication tolerance (2017).

[Childs 2008] H. R. Childs and D. J. Bremer, "Documentation of High Impact Visualizations and Improvement Plans for Utilization of VisIt for Reactor Simulation", LLNL-TR-407624, October 8, 2008.

[Fischer 2008] P. F. Fischer, J. W. Lottes, and Stefan G. Kerkemier, Nek5000 Web Page, http://nek5000.mcs.anl.gov, 2008.

[Grandy 2013] C. Grandy, T.K. Kim, et al, "Advanced Fast Reactor - 100 - Design Overview”, Proceedings of FR-13: Fast Reactor and Related Fuel Cycles, Paris, France, March 4-7, 2013.

[Hader 2014] J. S. Hader, E. R. Shemon and C. H. Lee, "Initial Verification of Heterogeneous Multigroup Cross Sections in MC2-3/PROTEUS", Transactions of ANS Winter Meeting 2014, Anaheim, CA, November 9-13, 2014.

[Hardie 1969] R. W. Hardie, W. W. Little , Jr. , "1DX: A One-Dimensional Diffusion Code for Generating Effective Nuclear Cross Sections", BNWL-954, Battelle Northwest Laboratory (1969)

[Hu 2013] Rui Hu, Thomas H. Fanning, A momentum source model for wire-wrapped rod bundles - Concept, validation, and application, In Nuclear Engineering and Design, Volume 262, 2013, Pages 371-389.

[Jarrett 2016] M. G. Jarrett, E. R. Shemon and C. H. Lee, "Heterogeneous Multigroup Cross Sections for Fast Reactor Calculations with MC2-3/PROTEUS", Proceedings of PHYSOR 2016, Sun Valley, ID, May 1-5, 2016. 
[Jeong 1995] Jeong, J., Hussain, F., 1995. On the identification of a vortex. J. Fluid Mech. 285, 69-94.

[Ku 1994] J. Y. Ku, L. K. Chang and D. Mohr, “Analysis of IFR Driver Fuel Hot Channel Factors," Fourth International Topical Meeting on Nuclear Thermal Hydraulics, Operation and Safety, Taipei, Taiwan, April 5-8, 1994.

[Kim 2012] T. K. Kim, C. Grandy, and B. Hill, "A 100 MWe Advanced Sodium-Cooled Fast Reactor Core Concept," PHYSOR 2012 - Advances in Reactor Physics - Linking Research, Industry, and Education, Knoxville, Tennessee, USA, April 15-20, 2012.

[Lee 2012] C. H. Lee and W. S. Yang, "MC2-3: Multigroup Cross Section Generation Code for Fast Reactor Anaysis," ANL/NE-11-41 Rev. 1, Argonne National Laboratory, January, 2012.

[Little 1969] W. W. Little, R. W. Hardie, “2DB User's Manual - Revision I”, BNWL-831, Rev. I, Battelle Pacific Northwest Laboratory (1969).

[Mahadevan 2014] V. S. Mahadevan, et al, "SHARP Coupled Multiphysics Toolkit for Reactor Analysis," Proceedings of ICAPP 2014, Charlotte, NC, April 6-9, 2014.

[Merzari 2008] E. Merzari, H. Ninokata, E. Baglietto, Numerical simulation of flows in tightlattice fuel bundles, In Nuclear Engineering and Design, Volume 238, Issue 7, 2008, Pages 1703-1719, ISSN 0029-5493.

[Merzari 2015] E. Merzari, E. Shemon, Y. Yu, J. Thomas, A. Obabko, R. Jain, V. Mahadevan, J. Solberg, R. Ferencz, R. Whitesides et al, "Multi-Physics Demonstration Problem with the SHARP Reactor Simulation Toolkit", Argonne National Laboratory Technical Report ANL-NE-15/44, December 21, 2015.

[Muraoka 1976] J. Muraoka, et al, “Assessment of FFTF Hot Channel Factors,” HEDL-TI-75226 (1976).

[Mynatt 1973] F. B. Mynatt, et. al., "The DOT III Two Dimensional Discrete Ordinates Transport Code," ORNL-TM-4280 (1973).

[Palmiotti 1995] G. Palmiotti, E. Lewis, C. Carrico, "VARIANT: VARIational Anisotropic Nodal Transport for Multidimensional Cartesian and Hexagonal Geometry Calculation," Argonne National Laboratory ANL-95/40, 1995.

[Shemon 2014] E. R. Shemon, C. H. Lee, M. A. Smith and A. Marin-Lafleche, "NEAMS Neutronics: Development and Validation Status", Proceedings of ICAPP 2014, Charlotte, NC, April 6-9, 2014.

[Sprague 2010] M. A. Sprague, "A comparison of Nek5000 and OpenFOAM for the DNS of Turbulent Channel Flow”, Presentation at Nek5000 Users Meeting, Argonne National Laboratory, December 10, 2010.

[Toppel 1983] B. J. Toppel, "A User's Guide to the REBUS-3 Fuel Cycle Analysis Capability," ANL-83-2, Argonne National Laboratory (1983).

[Waltar 1981] A. E. Walter, A. B. Reynolds, "Fast Breeder Reactors," Pergamon Press (1981).

[Waltar 2012] A. Walter, Fast Spectrum Reactors, Springer, New York (2012) 
[Yang 1995] W. S. Yang and A. M. Yacout, "Assessment of the SE2-ANL Code Using EBR-II Temperature Measurements", Proceedings of NURETH-7, Saratoga Springs, NY, September 10-15, 1995.

[Yang 2005] W. S. Yang, "Potential Gains through Reduced Hot Spot Factors," ANL Intralaboratory memo (2005)

[Yu 2011] Y.Q. Yu, B.H. Yan, X. Cheng, H.Y. Gu, Simulation of turbulent flow inside different subchannels in tight lattice bundle, In Annals of Nuclear Energy, Volume 38, Issue 11, 2011, Pages 2363-2373.

[Yu 2015] Yiqi Yu, Elia Merzari, Aleksandr Obabko, Justin Thomas, A porous medium model for predicting the duct wall temperature of sodium fast reactor fuel assembly, Nuclear Engineering and Design,295:48-58,2015 


\section{APPENDIX A. AFR-100}

The AFR-100 was developed to target emerging markets where a clean, secured and stable electricity resource is required but a large-scale plant is not accommodated. The AFR is aimed to be sized for local small grids, transportable from pre-licensed factories to the remote plant site for affordable supply, and able to operate for a long period without frequent refueling. To achieve these strategic goals, several design requirements have been proposed for the AFR core development; a power rating of $100 \mathrm{MWe}$ which is equivalent to $250 \mathrm{MWth}$ with the target thermal efficiency of $40 \%$, a long refueling interval with small burnup reactivity swing, a core barrel diameter of less than $3.0 \mathrm{~m}$, and an active core height of less than $1.5 \mathrm{~m}$.

The ANL suite of fast reactor analysis code system was used for the neutronics design. Fuel cycle analysis was performed with the DIF3D/REBUS-3 code system [Palmiotti 1995, Toppel 1983]. Region-dependent 21-group neutron cross section sets generated for a typical metallic fueled sodium-cooled fast reactor under the USDOE Integral Fast Reactor (IFR) program were utilized in this study. Using three-dimensional hexagonal-z geometry model, fuel cycle analyses were performed. Material thermal expansion at operating condition is modeled by adjusting the hexagonal pitch, axial meshes, and the fuel and structure volume fractions appropriately. The radial and axial thermal expansions were assumed to be the same as SS-316 (0.615\%) and HT-9 (0.731\%).

The U-10Zr binary metallic fuel was used as the primary fuel form of the AFR-100 because of its potential inherent safety features, favorable behavior for a long refueling interval, and because it is rapidly deployable. For accommodating the irradiation induced swelling, the binary metallic fuel has smeared density fuel of $75 \%$, and the swelling was modeled by a uniform axial growth of $8 \%$ in the core design.

Reactivity feedback coefficients and reactor kinetics parameters are calculated by positioning the primary control assemblies at the critical location. The coolant, fuel, and structural material density coefficients and the sodium void worth are determined using the VARI3D perturbation code [Adams 1975]. The first-order perturbation theory option is used for density coefficients, while the exact perturbation theory option is employed for the coolant void worth. The effective delayed neutron fraction and prompt neutron lifetime are also calculated using the VARI3D code. The radial and axial expansion coefficients and the control assembly worth are determined by the direct differences in the eigenvalues for the base and perturbed conditions using the DIF3D code.

Reactor orifice zoning and flow allocation were determined through steady-state thermalhydraulic analyses. Heating rates for thermal-hydraulic calculations were determined by coupled neutron and gamma heating calculations. Region-dependent 9-group neutron cross section set and 21-group gamma cross section set were used along with a gamma production matrix generated with NJOY and SDX codes in 9 neutron groups and 21 gamma groups. Neutron and gamma flux calculations were performed using the triangular-z finite difference option of the DIF3D code. Following the neutron flux calculation, the gamma source distribution was determined with the GAMSOR [Adams 1987] code. Using this gamma source distribution, the gamma flux calculation 
was performed as a fixed source problem. The neutron heating distribution was determined by multiplying the neutron flux by the neutron KERMA factors, and the gamma heating distribution is determined by multiplying the gamma flux by the gamma KERMA factors. The total heating rate was appropriately normalized to the reactor power.

The sub-channel analysis code SE2-ANL was employed for whole core temperature calculations. SE2-ANL is a modified version of the SUPERENERGY-2 [Beitel 1984] thermalhydraulic code interfaced with Argonne heating calculations. The SUPERENERGY-2 code is a multi-assembly, steady-state sub-channel analysis code designed specifically to perform efficient calculations of the detailed core-wide coolant temperature profiles in sodium cooled fast reactor core geometries. At Argonne, reactor hot spot analysis methods as well as fuel and cladding temperature calculation models have been added to the original version of SUPERENERGY-2. Both nominal and $2 \sigma$ temperatures are calculated for the fuel and cladding. The nominal fuel and cladding temperatures are obtained by solving a steady-state one-dimensional conduction equation. The $2 \sigma$ temperatures are calculated using the semi-statistical method.

Figures A.1 and A.2 show the radial and axial core layouts of the AFR-100 core, respectively, and detailed design parameters are provided in Ref. [Grandy 2013].

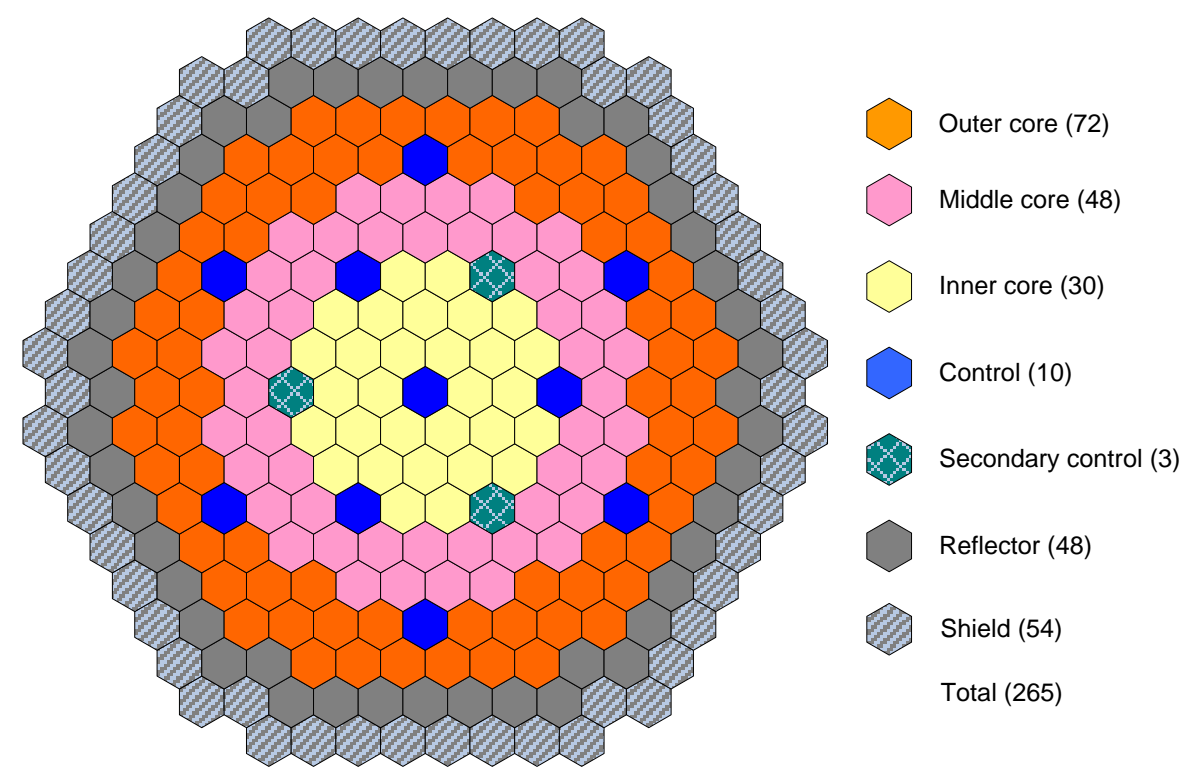

Figure A.1 Core radial layout of AFR-100 


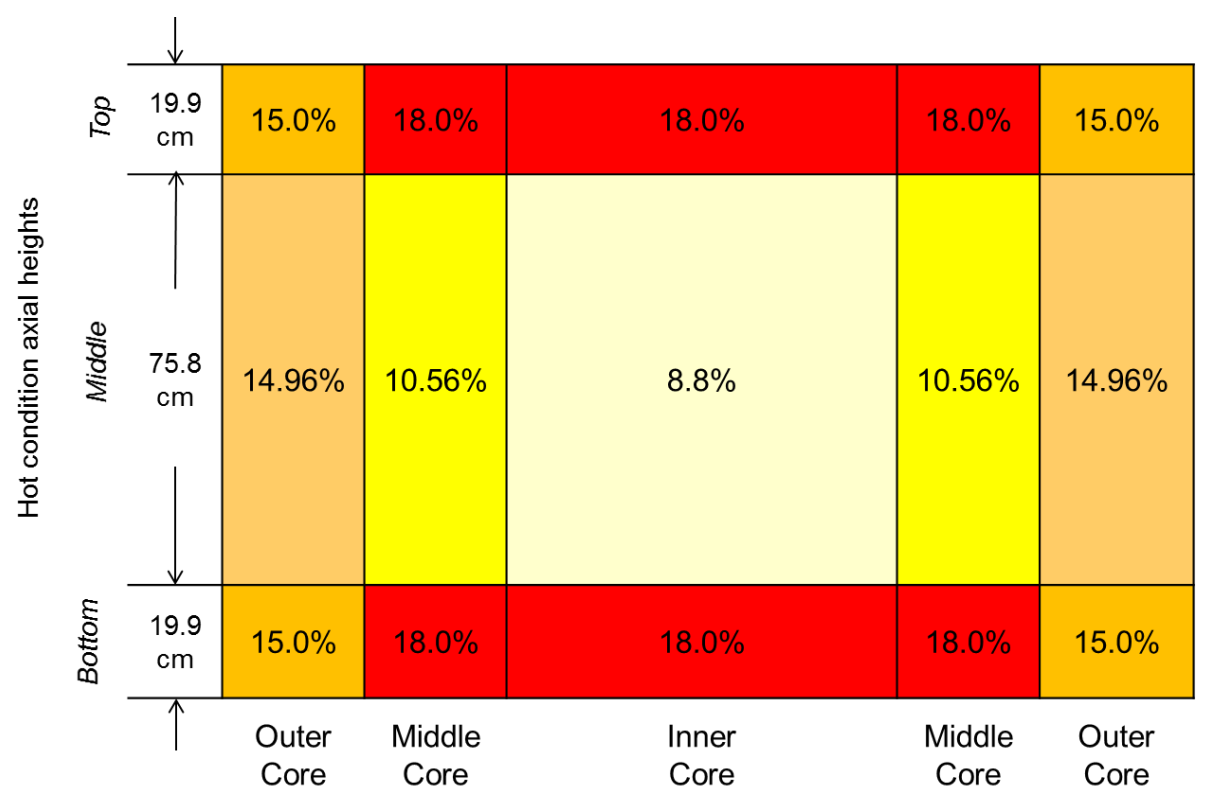

Figure A.2 Core axial layout and uranium enrichment distribution

The AFR-100 core adopted an onion-type enrichment zoning concept that was developed for a long refueling scheme and flat radial power distribution. The active core is divided into three different enrichment zones radially, and each zone has multiple zones axially. The average uranium enrichment is $11.97 \%$ for the inner core, $13.12 \%$ for the middle core, and $14.97 \%$ for the outer core.

Table A.1 provides the major core performance parameters of the AFR-100. The core can maintain criticality for 30 years by employing the unique enrichment zoning strategy of the AFR100 with low reactivity swing during the reactor life time. The average discharge burnup is 101 $\mathrm{MWd} / \mathrm{kg}$ and the peak discharge burnup is $172 \mathrm{MWd} / \mathrm{kg}$.

Table A.1 Core performance parameters.

\begin{tabular}{|l|l|}
\hline Parameters & AFR-100 \\
\hline Thermal power, MWt & 250 \\
\hline Refueling interval, year & 30 \\
\hline Number of batches & 1 \\
\hline Fuel form & $\mathrm{U}-\mathrm{Zr}$ \\
\hline Active core height, cm & 110 \\
\hline Average power density, kW/1 & 64.3 \\
\hline
\end{tabular}




\begin{tabular}{|l|l|}
\hline Fuel pin overall length, cm & 222 \\
\hline Initial heavy metal loading, $\mathrm{t}$ & 23.9 \\
\hline Specific power density, MW/t & 10.5 \\
\hline Average enrichment, $\%$ & 13.7 \\
\hline Burnup reactivity swing, $\% \Delta \mathrm{k}$ & 1.1 \\
\hline Overall fissile breeding Ratio & 0.80 \\
\hline Average discharge burnup, MWd/kg & 101 \\
\hline Peak discharge burnup, MWd/kg & 172 \\
\hline Peak fast fluence, $10^{23} \mathrm{n} / \mathrm{cm}{ }^{2}$ & 5.97 \\
\hline Linear heat rate (average/peak), kW/, & $15.2 / 28.2$ \\
\hline
\end{tabular}

Figure A.3 shows the power distribution in MW per each assembly at BOC and EOC. The peak radial power at BOC occurs in the sixth row, which is in the outer core zone, but the location moves to the second row, which is in the inner core. Figures A.4 and A.5 show the peak cladding inner wall temperatures and the minimum margins to fuel melt, respectively, with $2 \sigma$ hot channel factors that were employed in the CRBR core design. In the AFR-100, the flow rate of each assembly was determined to have flat coolant exit temperatures, and the calculated cladding and fuel temperatures were affected by the flow rates. The overall peak $2 \sigma$ cladding inner wall temperature is $693^{\circ} \mathrm{C}$. The overall minimum margin to fuel melt is higher than $420{ }^{\circ} \mathrm{C}$.

It is noted that all design parameters were calculated using the ANL legacy tools, and significant reductions of the peak cladding and fuel temperatures are expected when the parameters are reevaluated using the SHARP toolkits and newly developed HCFs in this study. 


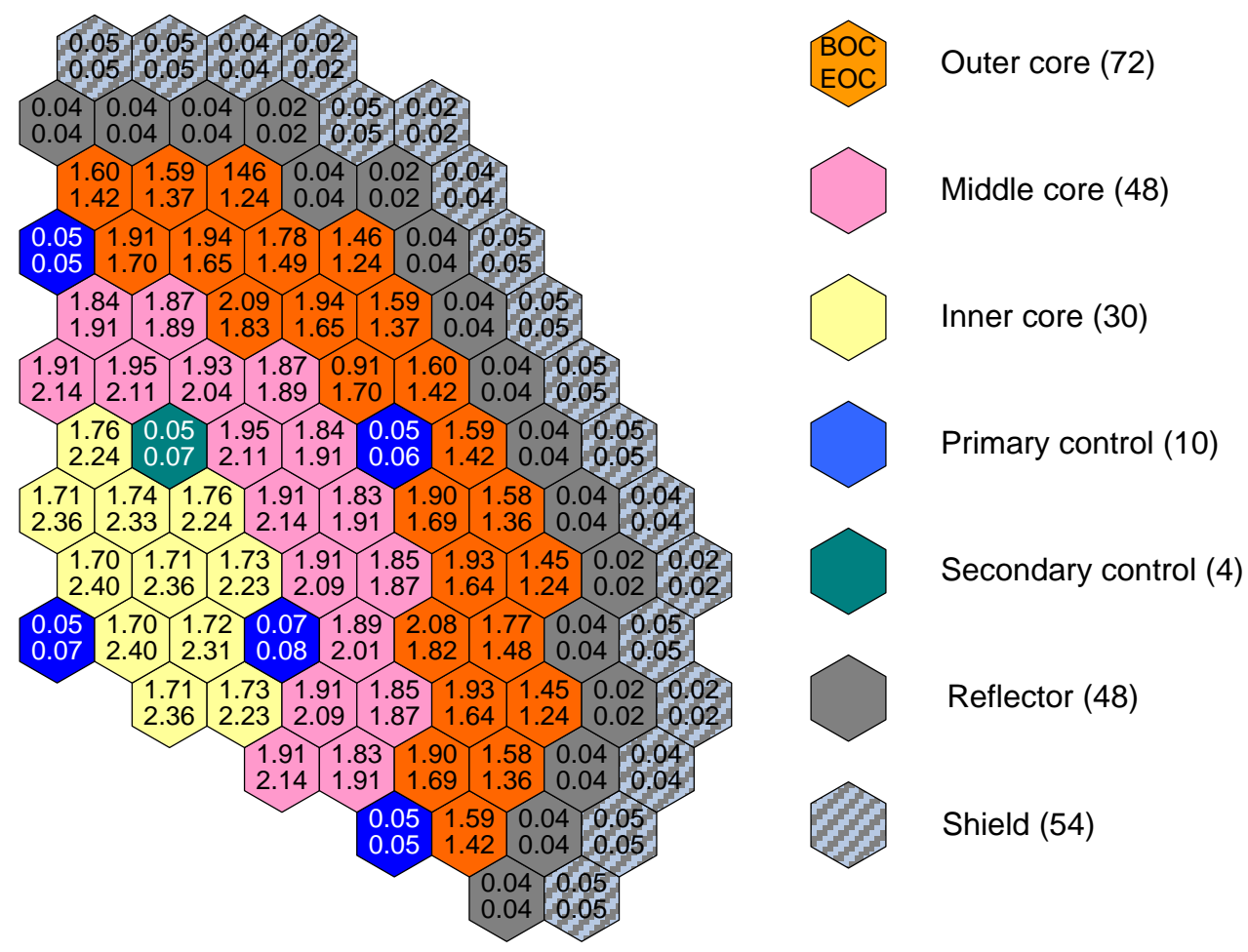

Figure A.3 Radial power distribution (MW per assembly in 1/3 Core).

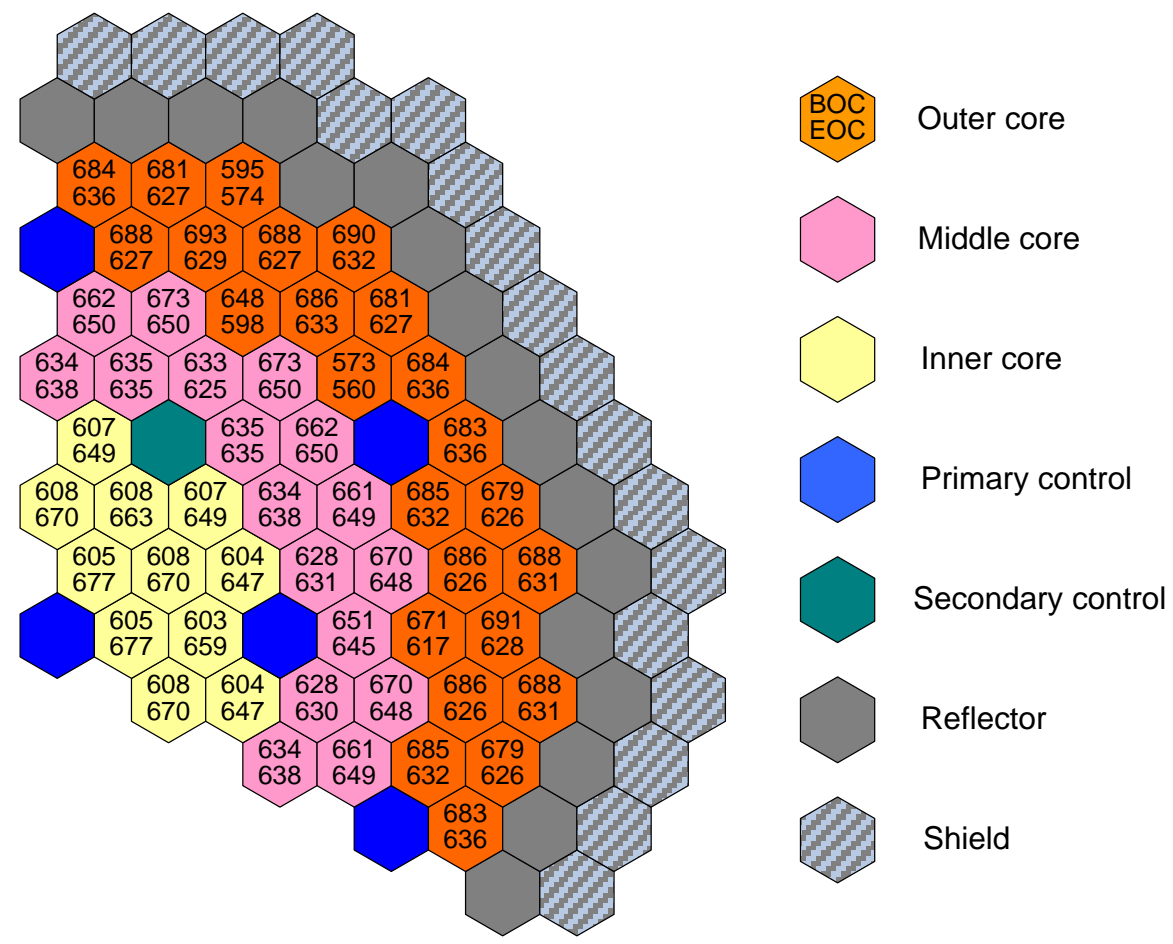

Figure A.4 Peak $2 \sigma$ cladding inner wall temperatures $\left({ }^{\circ} \mathrm{C}\right)$. 


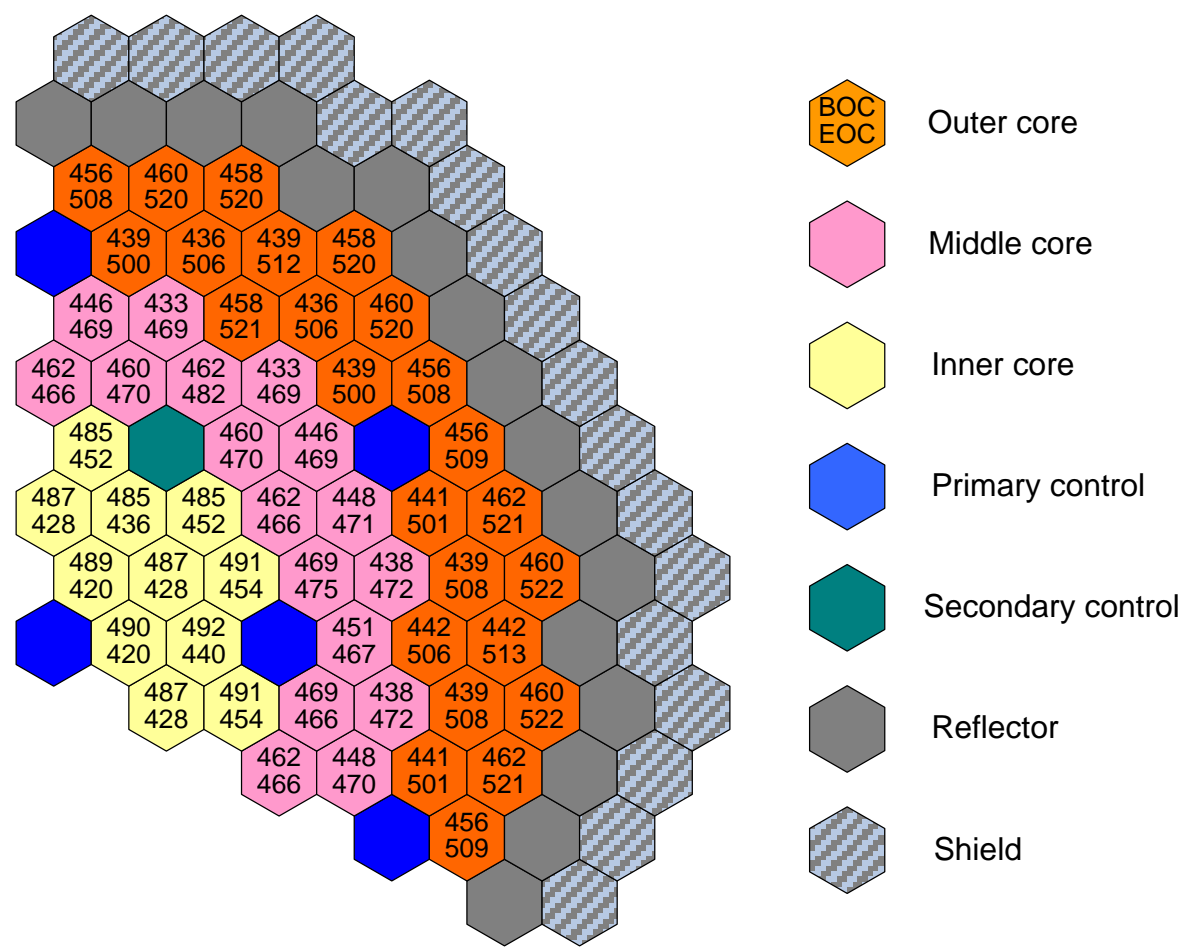

Figure A.5 Minimum $2 \sigma$ margins to fuel melt $\left({ }^{\circ} \mathrm{C}\right)$ 


\section{APPENDIX B. PROTEUS SENSITIVITY STUDIES}

Before performing multi-physics calculations with the Nek5000 code, a series of neutronics sensitivity studies were performed to identify optimal discretization parameters to ensure accuracy and efficiency. The PROTEUS neutronics approach, models, and results are discussed in this section.

Unlike computational fluid dynamics simulations, heterogeneous neutronics simulations require a comprehensive description of material compositions, densities, and interaction cross sections in addition to the detailed geometry input. Significant time was spent developing the detailed material densities for the hot configuration (assuming 0.615\% SS316 and 0.731\% HT9 thermal expansion factors, $8 \%$ fuel axial swelling, fuel radial swelling contacts hot inner cladding radius, displaced sodium bond to $27 \mathrm{~cm}$ above active core, $\mathrm{B}_{4} \mathrm{C}$ swelling ignored in lower shield). Specific neutronics approximations include replacement of fission gas in plenum with helium, geometrical homogenization of helium into cladding, and smearing the wire wrap into the cladding. The hot radial and axial dimensions are summarized in Table B.1 and Table B.2.

Table B.1 Hot radial dimensions for AFR-100 fuel assembly

\begin{tabular}{|l|l|}
\hline Assembly Parameter & Value at Hot Condition \\
\hline Assembly Pitch & $16.6176 \mathrm{~cm}$ \\
\hline Duct Flat-Flat & $16.2996 \mathrm{~cm}$ (outer) \\
& $15.6959 \mathrm{~cm}$ (inner) \\
\hline Pin Pitch & $1.6121 \mathrm{~cm}$ \\
\hline Clad Radii & $0.7516 \mathrm{~cm}$ (outer w/ smeared WW) \\
& $0.7496 \mathrm{~cm}$ (outer bare bundle) \\
& $0.6993 \mathrm{~cm}$ (inner) \\
\hline Elevation & $292.9491 \mathrm{~cm}$ (total) \\
& $96.6198 \mathrm{to} 216.1504 \mathrm{~cm}$ (active core) \\
\hline Pin Power & $18.315 \mathrm{~kW} /$ pin \\
\hline
\end{tabular}

Table B.2 Hot axial dimensions for AFR-100 fuel assembly

\begin{tabular}{|l|l|}
\hline Axial Zone & Lower Z $(\mathrm{cm})$ \\
\hline Lower Structure & 0.0 \\
\hline Lower Shield & 30.2139 \\
\hline Lower Reflector & 63.4169 \\
\hline Inner Bottom Core & 96.6198 \\
\hline Inner Middle Core & 120.4933 \\
\hline Inner Top Core & 196.2649 \\
\hline $\begin{array}{l}\text { Vented Gas Plenum with } \\
\text { Displaced Sodium Bond }\end{array}$ & 216.1504 \\
\hline
\end{tabular}




\begin{tabular}{|l|l|}
\hline $\begin{array}{l}\text { Vented Gas Plenum with } \\
\text { Fission Gas }\end{array}$ & 243.3153 \\
\hline Upper Structure & 262.7352 \\
\hline
\end{tabular}

\section{B.1 Multigroup Cross Section Generation}

Multigroup cross section generation is the first step in performing any deterministic neutronics simulation. This step involves condensing the material interaction cross section data (a highly complex function of energy for each isotope and reaction type) into constants associated with broad energy group ranges that can be input to the deterministic transport solver. This is done by weighting the continuous energy data with the expected neutron flux in the energy group to preserving the total reaction rate in the energy group. The presence of heterogeneous geometry features (pin-by-pin representation, assembly duct, etc) increases the difficulty of generating accurate multigroup cross sections because the local neutron flux in each spatial region is difficult to accurately determine without advanced tools.

In this work, the $M^{2}-3$ advanced cross section generation code [Lee 2012] in conjunction with TWODANT [Alcouffe 1984] was used to generate multigroup constants due to its ability to account for heterogeneity effects. Prior to running these codes, the material compositions were determined for hot conditions by taking the manufactured compositions, converting natural elements into isotopics (needed by $\mathrm{MC}^{2}-3$ ), and smearing the composition over the expanded hot volumes. Then, homogenized compositions for each axial segment (needed only for cross section generation procedures) were calculated based on the hot volume fractions.

The workflow used for $\mathrm{MC}^{2}-3 / \mathrm{TWODANT}$ is shown in Figure B.1.1 and is based on previous experience generating multigroup cross sections for highly heterogeneous geometries [Hader 2014], [Jarrett 2016]. An infinite medium homogeneous mixture for each axial composition was simulated in $\mathrm{MC}^{2}-3$ to yield 1041-group cross sections. These compositions were then used in a TWODANT R-Z radially-homogenized model (either 7-pin or 91-pin) to capture the axial leakage effect in 3D. The resulting 1041-group region dependent fluxes were used to collapse the 1041group cross sections for the non-fuel axial zones to obtain heterogeneous cross sections. For axial zones containing active fuel, new 1041-group cross sections including pin radial heterogeneity effects were generated by converting the 7-pin or 91-pin geometry into 1-D cylindrical geometry in $\mathrm{MC}^{2}-3$ while preserving volume fractions of each material to model the explicit fuel, cladding and sodium materials. Such a conversion is depicted in Figure B.1.2 (rings are not to scale or correct in number). The resulting fuel zone 1041-group cross sections were then collapsed with the TWODANT 1041-group fluxes. 


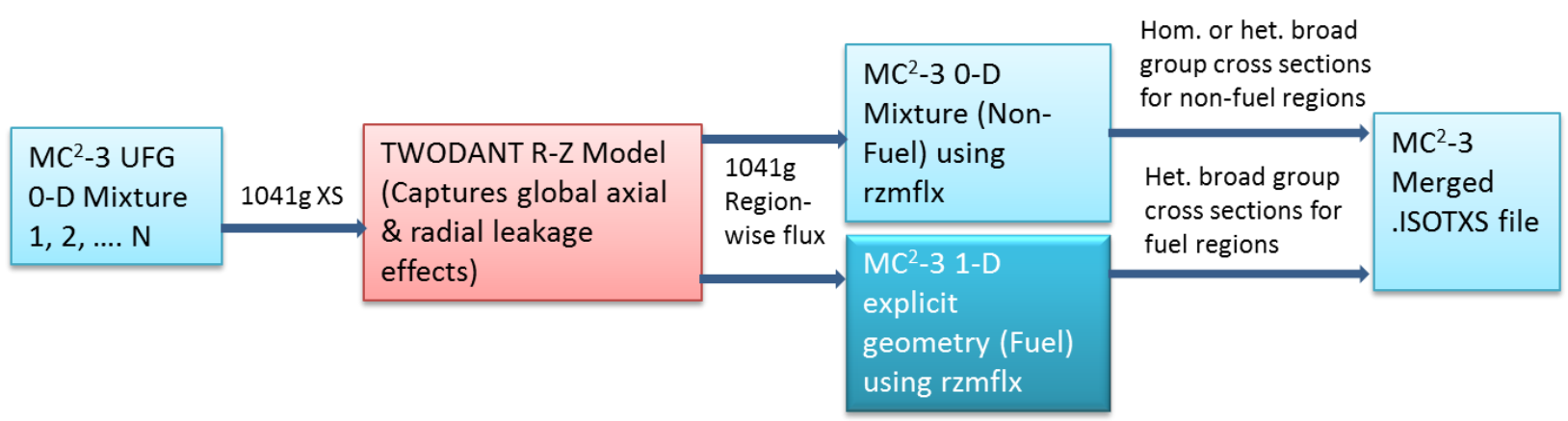

Figure B.1.1 Cross section generation procedure for heterogeneous 3D pin configurations

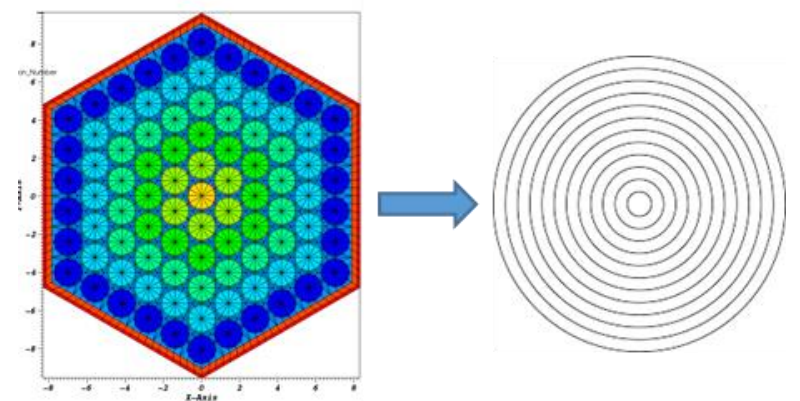

Figure B.1.2 General depiction of assembly into 1-D cylinder geometry (not to scale)

Broad group (4-group, 9-group, 33-group, 70-group) cross sections configurations were generated including assembly duct, inter-assembly sodium gap, and 6 rings of pins for the 91-pin assembly calculations at two temperatures $(300 \mathrm{~K}$ and $1000 \mathrm{~K})$ with specific naming conventions according to the PROTEUS temperature interpolation requirements.

\section{B.2 Mesh Preparation}

When preparing meshes for coupled calculations using the SHARP toolkit, the H5M (MOAB) mesh format is required for all physics modules. The offline coupling method used in this work removes this restriction, so each physics module can use its own mesh generator and mesh format. The UFMesh format from the PROTEUS MeshTools suite was chosen to generate the PROTEUS meshes as it easily incorporates repeated pin cell features in a hexagonal geometry, as well as assembly ducts. Meshes of varying degrees of fidelity were generated for both the 7-pin and 91-pin configurations in order to perform mesh convergence studies. The azimuthal intervals (A) and radial intervals $(\mathrm{R})$ for the fuel pins can be varied independently of each other, and the number of axial planes can also be varied. Several 2D meshes for the 7-pin configuration are shown in Figure B.2.1 with variable azimuthal (A) and radial (R) intervals. For PROTEUS-SN, a 3D finite element mesh is required. For PROTEUS-MOCEX, a 2D finite element mesh is required, along with the axial plane discretization provided separately. 


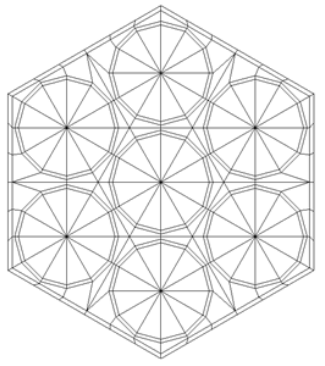

A1R1

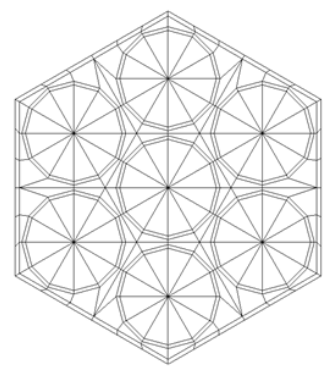

A1R1

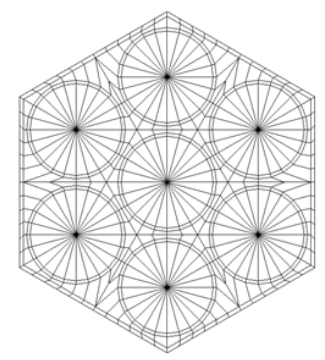

A2R1

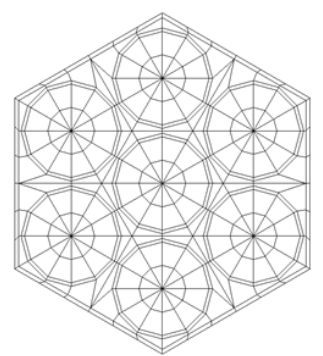

A1R2

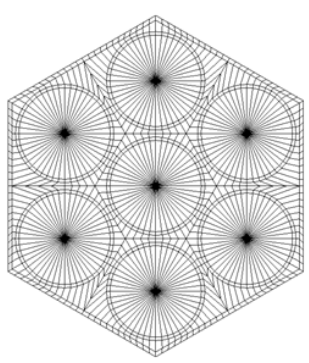

A4R1

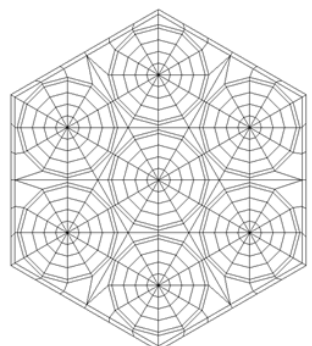

A1R4

Figure B.2.1 Visualization of various neutronics meshes for the 7-pin configuration

\section{B.3 Transport Solvers: PROTEUS-SN vs. PROTEUS-MOCEX}

The original intention of this analysis was to use the PROTEUS-SN solver so that the offline coupling method could be compared to online coupling method using the SHARP toolkit. However, the 7-pin configuration studied in this work presented serious challenges for the PROTEUS-SN solver. Slow performance with reflective boundary conditions is a known problem with PROTEUSSN because the preconditioner used in PETSc cannot properly precondition the reflective boundary condition part of the matrix. For larger domains such as the 91-pin configuration, the relative burden of the reflected boundary conditions becomes smaller, but the 7-pin case is quite small and thus, ironically, the effect is more severe. To understand this from a physics perspective, numerous angles are coupled together on the reflected boundary surface which require the definition of a preconditioner that handles the full angular connections (this was attempted in the past, but failed to scale in parallel). Without a proper preconditioner (acceleration), the time required to converge the angular boundary conditions is prohibitive. DIF3D-VARIANT, for example, also shows poor convergence behavior for optically thin, infinite Cartesian problems. Most first order discrete ordinates $(\mathrm{SN})$ codes have serious problems with this issue and some actually forbid it by only allowing 3 reflected boundary conditions. It should be noted that while thermal hydraulics codes like Nek5000 can compute the result to this infinite problem by ignoring the connection to all other assemblies, PROTEUS-SN cannot ignore these connections and was not built to run efficiently on infinitely large problems. 
Therefore, the PROTEUS-MOCEX neutronics solver was chosen to solve both the 7-pin and 91-pin configurations because it does not suffer from convergence issues due to reflective boundary conditions. Additionally, for highly heterogeneous geometry, the PROTEUS-MOCEX solver is expected to yield a more accurate solution then PROTEUS-SN due to its different methodology (discontinuous Galerkin/MOC vs. continuous FEM). A limited number of PROTEUS-SN calculations are performed for the 91-pin configuration in order to draw comparisons to the PROTEUS-MOCEX solver. The two solvers are briefly summarized in Table B.3.1.

Table B.3.1 Comparison of PROTEUS-SN and PROTEUS-MOCEX neutronics solvers

\begin{tabular}{|l|l|l|}
\hline Features & PROTEUS-SN & PROTEUS-MOCEX \\
\hline Formulation & $\begin{array}{l}2^{\text {nd }} \text { order even parity } \\
\text { formulation with continuous } \\
\text { finite elements }\end{array}$ & $\begin{array}{l}\text { 2D method of characteristics } \\
\text { + discontinuous Galerkin } \\
\text { finite elements in Z } \\
\text { dimension }\end{array}$ \\
\hline Multiphysics & $\begin{array}{l}\text { SHARP coupling, } \\
\text { deformation, temperature and } \\
\text { density feedback }\end{array}$ & $\begin{array}{l}\text { T/H module in progress, } \\
\text { temperature interpolation } \\
\text { possible with input file } \\
\text { manipulation }\end{array}$ \\
\hline Mesh Discretization & $\begin{array}{l}\text { 3D unstructured finite } \\
\text { element }\end{array}$ & $\begin{array}{l}\text { 2D unstructured finite } \\
\text { element extruded in the } 3^{\text {rd }} \\
\text { dimension }\end{array}$ \\
\hline Angle Discretization & Discrete ordinates & Discrete ordinates \\
\hline Energy Discretization & Multigroup & Multigroup \\
\hline Heterogeneous Problems & $\begin{array}{l}\text { Better for homogeneous and } \\
\text { partially heterogeneous } \\
\text { systems (explicit duct wall, } \\
\text { but not explicit pins) }\end{array}$ & $\begin{array}{l}\text { Ideal for fully heterogeneous } \\
\text { systems }\end{array}$ \\
\hline $\begin{array}{l}\text { Performance with Reflected } \\
\text { Boundary Conditions }\end{array}$ & $\begin{array}{l}\text { Severe convergence issues for } \\
\text { optically thin domains }\end{array}$ & No convergence issues \\
\hline
\end{tabular}

\section{B.4 Mesh, Angle and Energy Convergence Studies}

A series of mesh, angular, and energy convergence studies was performed in the following section. This step is always necessary in deterministic neutronics simulations, and especially worthwhile to guarantee the accuracy of the 7-pin bare bundle and 91-pin bare bundle nominal condition simulations for hot channel factor, which will be re-used to provide power profiles to Nek5000. A uniform $300 \mathrm{~K}$ temperature distribution was used for the convergence studies. 


\section{B.4.1 Axial Mesh Refinement}

The 7-pin and 91-pin configurations have identical fuel pin axial geometry as listed in Table B.2. The only difference between the two configurations, axially, is the extra duct and interassembly sodium gap present in the 91-pin configuration which extend the entire axial length.

Axial refinement studies were performed for the 7-pin geometry. The axial active fuel zones (inner bottom, inner middle, and inner top core) were subdivided into $<5 \mathrm{~cm}$ axial intervals already in order to apply different temperatures in future calculations. Several calculations with MOCEX were performed with different axial subdivisions among non-fuel zones as well as further refinement of the fuel zone (156 plane case) as specified in axial division as specified in Table B.4.1.1. The group structure, temperature, cubature, and 2D mesh were held constant to isolate the axial discretization effects. It was determined at 78 axial subdivisions were optimal based on eigenvalue convergence. The same axial refinement can be assumed to apply for the 91-pin configuration.

Table B.4.1.1 MOCEX eigenvalues with varying degrees of axial refinement (7-pin)

\begin{tabular}{|l|l|l|l|l|l|l|}
\hline $\begin{array}{l}\text { Energy } \\
\text { Group }\end{array}$ & Eigenvalue & Temperature & $\begin{array}{l}\text { Angular } \\
\text { Cubature }\end{array}$ & $\begin{array}{l}\text { Mesh } \\
\text { Elements } \\
\text { (2D) }\end{array}$ & $\begin{array}{l}\text { Mesh } \\
\text { Refinement }\end{array}$ & $\begin{array}{l}\text { Number of } \\
\text { Planes }\end{array}$ \\
\hline 4 & 1.13044 & $300 \mathrm{~K}$ & L3T5 & 3061 & A1R1 & 36 \\
\hline 4 & 1.12976 & $300 \mathrm{~K}$ & L3T5 & 3061 & A1R1 & 54 \\
\hline 4 & 1.12980 & $300 \mathrm{~K}$ & L3T5 & 3061 & A1R1 & 78 \\
\hline 4 & 1.12980 & $300 \mathrm{~K}$ & L3T5 & 3061 & A1R1 & 156 \\
\hline
\end{tabular}

\section{B.4.2 Energy Group Refinement}

Energy group refinement studies were performed using 4-, 9-, 33-, and 70-group cross section data sets generated with the $\mathrm{MC}^{2-3 / T W O D A N T}$ procedure defined in Section B.1. The temperature, cubature, 2D mesh, and axial discretizations were held constant to isolate the energy group discretization effects.

Table B.4.2.1 presents the 7-pin eigenvalues as a function of energy groups. A minimum of 33 energy groups is necessary. The 70-group cross section set changes the eigenvalue by $-59 \mathrm{pcm}$, but, as shown in Figure 4.3.4.2.1 the normalized axial power does not significantly differ between the 33-group and 70-group calculations. This is reflected in the similar peak/average axial power reported in the table for the 2 calculations (differ by less than 1\%). Heterogeneous fast reactor problems typically do require the use of 33 or more energy groups.

Table B.4.2.1 MOCEX eigenvalues with varying group structures (7-pin) 


\begin{tabular}{|l|l|l|l|l|l|l|l|}
\hline $\begin{array}{l}\text { Energy } \\
\text { Group }\end{array}$ & Eigenvalue & $\begin{array}{l}\text { Peak/ } \\
\text { Average } \\
\text { Axial } \\
\text { Power }\end{array}$ & Temperature & $\begin{array}{l}\text { Angular } \\
\text { Cubature }\end{array}$ & $\begin{array}{l}\text { Mesh } \\
\text { Elements } \\
\text { (2D) }\end{array}$ & $\begin{array}{l}\text { Mesh } \\
\text { Refinement }\end{array}$ & Planes \\
\hline 4 & 1.12976 & 1.7982 & $300 \mathrm{~K}$ & L3T5 & 3061 & A1R1 & 78 \\
\hline 9 & 1.12902 & 1.9410 & $300 \mathrm{~K}$ & L3T5 & 3061 & A1R1 & 78 \\
\hline 33 & 1.12708 & 1.9364 & $300 \mathrm{~K}$ & L3T5 & 3061 & A1R1 & 78 \\
\hline 70 & 1.12649 & 1.9298 & $300 \mathrm{~K}$ & L3T5 & 3061 & A1R1 & 78 \\
\hline
\end{tabular}

Since the 70-group calculation significantly increases the computational requirements and has nearly the same peak/average power axially, the 33-group cross section set is satisfactory for future calculations. Note, only the power from the first ring was plotted in Figure B.4.2.1 because the power in both rings are nearly identical.

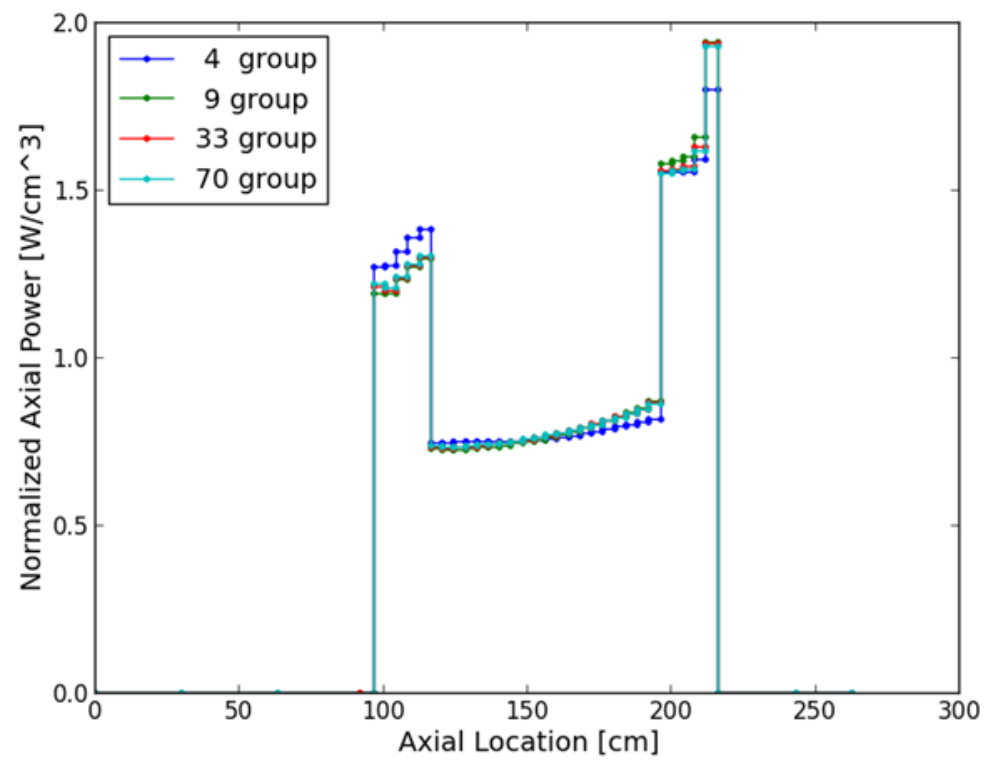

Figure B.4.2.1 MOCEX axial power profile with varying group structure (7-pin)

The axial power profile in Figure B.4.2.1 is unusual due to the onion-type enrichment pattern of the inner fuel $(18.0 \%$ enriched at the top and bottom zones and $8.8 \%$ in the middle zone) also described in Figure 3.1.2. A quick test was performed to give confidence in the model by assigning to the entire active fuel region a uniform $18.0 \%$ enrichment. The expected cosine shape for the uniform enrichment case is clearly seen in Figure B.4.3.2 using a 4-group model and confirms the material and geometry mapping. The power peaking seen in the upper core region (close to $216 \mathrm{~cm}$ axial height) is discussed in the main body of this report in Section 4.3.1. For now, we note that that the peak axial power is in the axial center of the core in the uniform enrichment case (roughly $125 \%$ of average axial power), whereas it is located at the top of the core in the actual AFR-100 
design (193\% of average axial power). This has significant impact on the outlet temperature distribution as will be seen in the Nek5000 calculation section.

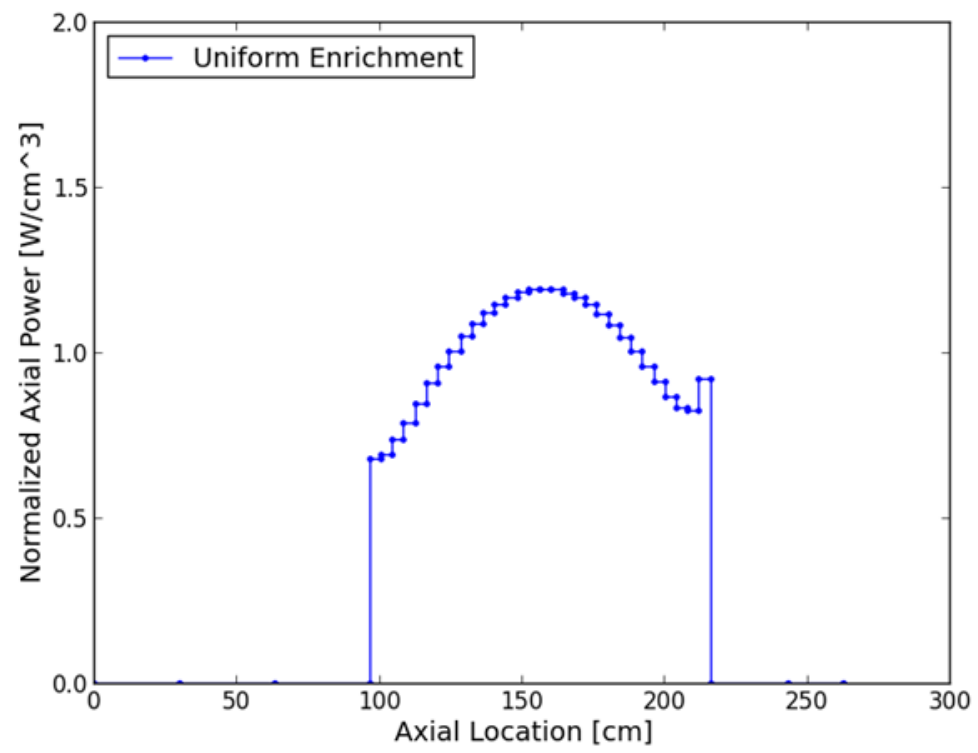

Figure B.4.2.2 MOCEX axial power profile for uniform enrichment problem (7-pin)

Energy group convergence studies were similarly performed for the 91-pin configuration. In this case, the difference in eigenvalue between 33-group and 70-group simulations is only $7 \mathrm{pcm}$. Inspection of Figure B.4.2.3 indicates the 33-group and 70-group power profiles (for the center pin) are almost identical. Thus, 33-group cross sections are sufficient for the 91-pin configuration. The difference in ring-wise power profiles is negligible as shown in Figure B.4.2.4. Therefore, the center-pin (Ring 1) profile is representative of all pins in the 91-pin geometry.

Table B.4.2.2 MOCEX eigenvalue results with varying group structures (91-pin)

\begin{tabular}{|l|l|l|l|l|l|l|}
\hline $\begin{array}{l}\text { Energy } \\
\text { Group }\end{array}$ & Eigenvalue & Temperature & $\begin{array}{l}\text { Angular } \\
\text { Cubature }\end{array}$ & $\begin{array}{l}\text { Mesh } \\
\text { elements } \\
(2 D)\end{array}$ & $\begin{array}{l}\text { Mesh } \\
\text { Refinement }\end{array}$ & Planes \\
\hline 4 & 1.09641 & $300 \mathrm{~K}$ & L3T5 & 3061 & A1R1 & 78 \\
\hline 33 & 1.10327 & $300 \mathrm{~K}$ & L3T5 & 3061 & A1R1 & 78 \\
\hline 70 & 1.10320 & $300 \mathrm{~K}$ & L3T5 & 3061 & A1R1 & 78 \\
\hline
\end{tabular}




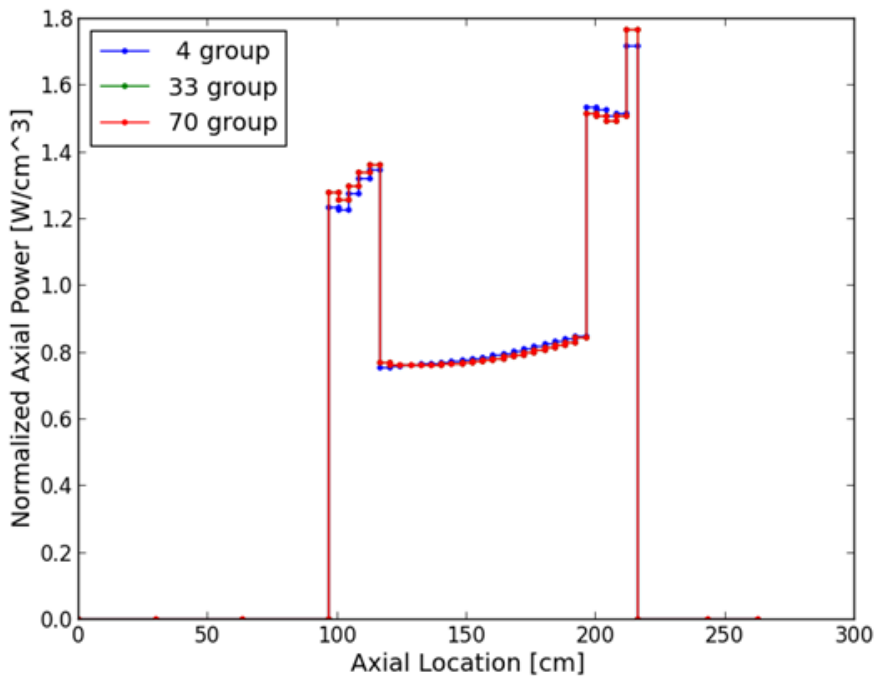

Figure B.4.2.3 MOCEX axial power profile with varying group structure (91-pin)

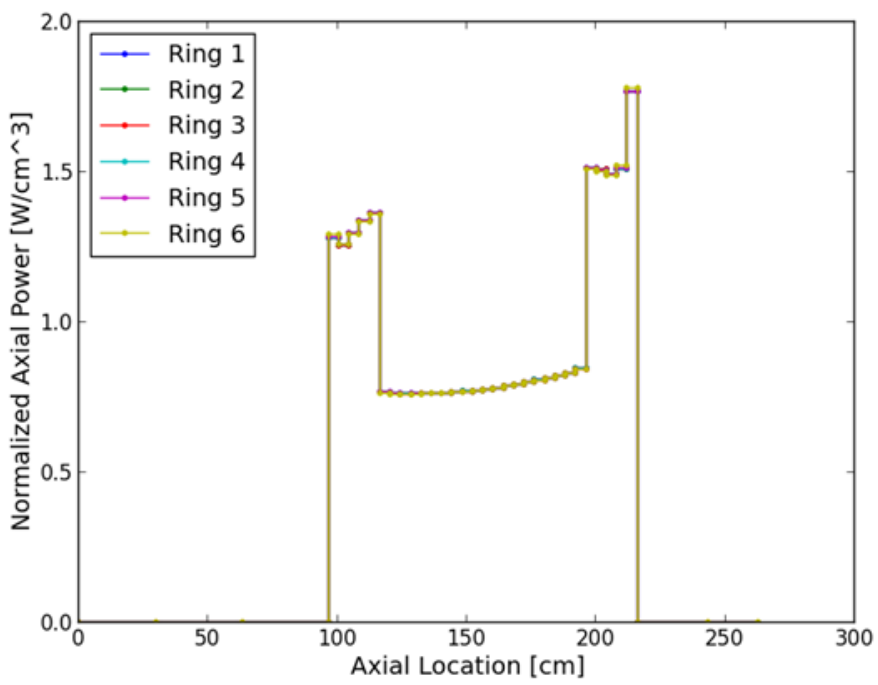

Figure B.4.2.4 MOCEX ring-wise axial power profiles with 33-groups (91-pin)

\section{B.4.3 Radial Mesh Refinement}

Radial mesh refinements studies were performed for the 7-pin configuration. The starting 2D mesh (denoted A1R1, indicating azimuthal order 1 and radial order 1) was refined by first increasing the azimuthal order while maintaining a constant value for the radial division of the fuel sections. The calculation was extremely insensitive to varying either the azimuthal order or increasing the number of radial subdivisions. We note that the results in the following tables used the coarse 4-group cross section set for quick throughput of calculations; however, the same trends 
typically apply for finer cross section sets so do not need to be repeated for the 33-group set. The converged radial mesh is depicted in Figure B.4.3.1.

Table B.4.3.1 MOCEX eigenvalue results with varying azimuthal subdivisions (7-pin)

\begin{tabular}{|l|l|l|l|l|l|l|}
\hline $\begin{array}{l}\text { Energy } \\
\text { Group }\end{array}$ & Eigenvalue & Temperature & $\begin{array}{l}\text { Angular } \\
\text { Cubature }\end{array}$ & $\begin{array}{l}\text { Mesh } \\
\text { Elements (2D) }\end{array}$ & $\begin{array}{l}\text { Mesh } \\
\text { Refinement }\end{array}$ & Planes \\
\hline 4 & 1.12980 & $300 \mathrm{~K}$ & L3T5 & 265 & A1R1 & 78 \\
\hline 4 & 1.12977 & $300 \mathrm{~K}$ & L3T5 & 529 & A2R1 & 78 \\
\hline 4 & 1.12977 & $300 \mathrm{~K}$ & L3T5 & 1057 & A4R1 & 78 \\
\hline 4 & 1.12977 & $300 \mathrm{~K}$ & L3T5 & 1585 & A6R1 & 78 \\
\hline
\end{tabular}

Table B.4.3.2 MOCEX eigenvalue results with varying radial subdivisions (7-pin)

\begin{tabular}{|l|l|l|l|l|l|l|}
\hline $\begin{array}{l}\text { Energy } \\
\text { Group }\end{array}$ & Eigenvalue & Temperature & $\begin{array}{l}\text { Angular } \\
\text { Cubature }\end{array}$ & $\begin{array}{l}\text { Mesh Elements } \\
\text { (2D) }\end{array}$ & $\begin{array}{l}\text { Mesh } \\
\text { Refinement }\end{array}$ & Planes \\
\hline 4 & 1.12980 & $300 \mathrm{~K}$ & L3T5 & 265 & A1R1 & 78 \\
\hline 4 & 1.12980 & $300 \mathrm{~K}$ & L3T5 & 529 & A1R2 & 78 \\
\hline 4 & 1.12978 & $300 \mathrm{~K}$ & L3T5 & 1057 & A1R4 & 78 \\
\hline
\end{tabular}

The 91-pin configuration displayed similar insensitivity to the radial mesh, as shown in Table B.4.3.3, despite the addition of the duct and inter-assembly sodium gap. The A1R1 mesh, depicted in Figure B.2.1, produces sufficiently converged results.

Table B.4.3.3 MOCEX results with varying radial and azimuthal subdivisions (91-pin)

\begin{tabular}{|l|l|l|l|l|l|l|}
\hline $\begin{array}{l}\text { Energy } \\
\text { Group }\end{array}$ & Eigenvalue & Temperature & $\begin{array}{l}\text { Angular } \\
\text { Cubature }\end{array}$ & $\begin{array}{l}\text { Mesh } \\
\text { Elements (2D) }\end{array}$ & $\begin{array}{l}\text { Mesh } \\
\text { Refinement }\end{array}$ & Planes \\
\hline 33 & 1.10327 & $300 \mathrm{~K}$ & L3T5 & 3061 & A1R1 & 78 \\
\hline 33 & 1.10323 & $300 \mathrm{~K}$ & L3T5 & 6121 & A2R1 & 78 \\
\hline 33 & 1.10324 & $300 \mathrm{~K}$ & L3T5 & 16609 & A4R2 & 78 \\
\hline
\end{tabular}

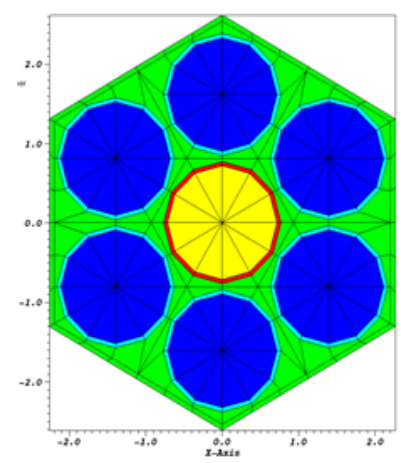

Figure B.4.3.1 Converged 2D neutronics mesh (265 elements) for the 7-pin configuration 


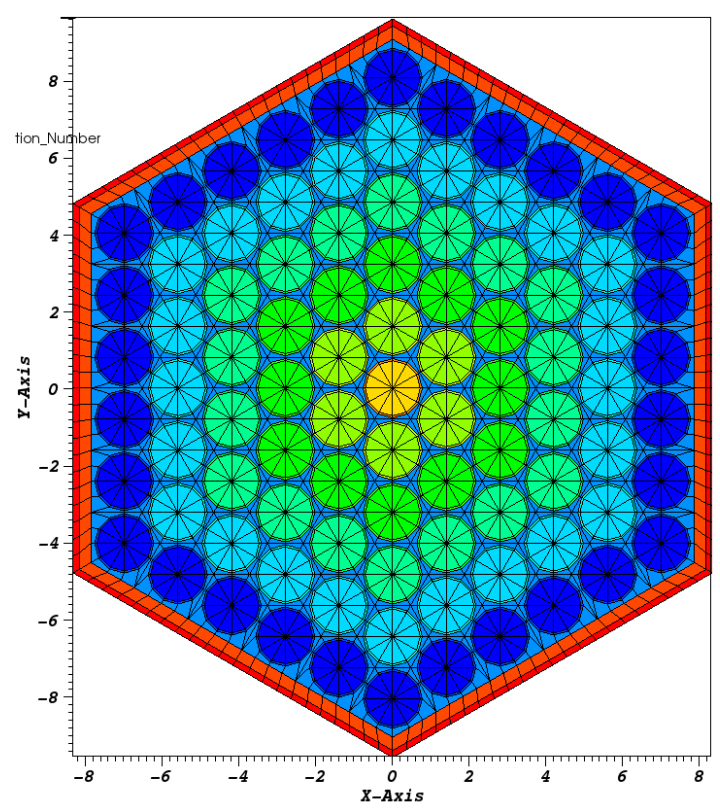

Figure B.4.3.2 Converged 2D neutronics mesh (3061 elements) for the 91-pin configuration

\section{B.4.4 Angular Cubature Refinement}

Angular cubature refinement studies are necessary for all deterministic codes using the discrete ordinate approximation. For this (and most) analysis the Legendre-Tchebychev product cubature is preferred because it allows the number of axial angular directions to be varied independently of the radial angular directions. The Legendre (L) order dictates the number of axial planes at which collections of Tchebychev angles are placed on the sphere. When reflected boundary conditions are applied, only certain sets of angular cubature are permitted which preserve the angular reflection off the planes present in the geometry. The L3T5, L7T11, and L7T17 cubatures meet these requirements (among others).

The MOCEX eigenvalue was not dependent on the cubature for the 7-pin case at all and therefore cubature order L3T5 is sufficient for this problem.

Table B.4.4.1 MOCEX eigenvalue results with varying angular cubature (7-pin)

\begin{tabular}{|l|l|l|l|l|l|l|}
\hline $\begin{array}{l}\text { Energy } \\
\text { Group }\end{array}$ & Eigenvalue & Temperature & $\begin{array}{l}\text { Angular } \\
\text { Cubature }\end{array}$ & $\begin{array}{l}\text { Mesh Elements } \\
(\text { 2D) }\end{array}$ & $\begin{array}{l}\text { Mesh } \\
\text { Refinement }\end{array}$ & Planes \\
\hline 4 & 1.12980 & $300 \mathrm{~K}$ & L3T5 & 265 & A1R1 & 78 \\
\hline 4 & 1.12980 & $300 \mathrm{~K}$ & L7T11 & 265 & A1R1 & 78 \\
\hline 4 & 1.12980 & $300 \mathrm{~K}$ & L7T17 & 265 & A1R1 & 78 \\
\hline
\end{tabular}

The 91-pin configuration was slightly more sensitive to cubature than the 7-pin configuration. Increasing the cubature from L3T5 to L7T11 yielded $+17 \mathrm{pcm}$, and another $+4 \mathrm{pcm}$ when increasing 
to L7T17. However, since the axial profile was nearly identical in all three cases, the dramatic increase in computational resources (61 core-hours for L3T5 vs. 252 core-hours for L7T17) was not justified, and L3T5 is recommended.

Table B.4.4.2 MOCEX eigenvalue results with varying angular cubature (91-pin)

\begin{tabular}{|l|l|l|l|l|l|l|}
\hline $\begin{array}{l}\text { Energy } \\
\text { Group }\end{array}$ & $\begin{array}{l}\text { Eigenval } \\
\text { ue }\end{array}$ & $\begin{array}{l}\text { Temperatur } \\
\mathrm{e}\end{array}$ & $\begin{array}{l}\text { Angular } \\
\text { Cubatur } \\
\text { e }\end{array}$ & $\begin{array}{l}\text { Mesh } \\
\text { Elements } \\
(2 \mathrm{D})\end{array}$ & $\begin{array}{l}\text { Mesh } \\
\text { Refinement }\end{array}$ & Planes \\
\hline 33 & 1.10327 & $300 \mathrm{~K}$ & L3T5 & 3061 & A1R1 & 78 \\
\hline 33 & 1.10344 & $300 \mathrm{~K}$ & L7T11 & 3061 & A1R1 & 78 \\
\hline 33 & 1.10348 & $300 \mathrm{~K}$ & L7T17 & 3061 & A1R1 & 78 \\
\hline
\end{tabular}

\section{B.4.5 Convergence Study Conclusions}

In summary, the PROTEUS-MOCEX calculation for both 7-pin and 91-pin configurations was insensitive to spatial resolution and angular cubature. A minimum of 33 energy groups should be used along with axial mesh subdivisions less than or equal to $5 \mathrm{~cm}$. Based on these observations, the energy, angle, and mesh discretization parameters listed in Table B.4.5.1 are recommended to obtain converged axial power profiles in subsequent hot channel factor calculations.

Table B.4.5.1 Recommended MOCEX discretization parameters

\begin{tabular}{|l|l|l|l|l|l|}
\hline Configuration & $\begin{array}{l}\text { Energy } \\
\text { Group }\end{array}$ & $\begin{array}{l}\text { Angular } \\
\text { Cubature }\end{array}$ & $\begin{array}{l}\text { Mesh } \\
\text { Elements } \\
(2 \mathrm{D})\end{array}$ & $\begin{array}{l}\text { Mesh } \\
\text { Refinement }\end{array}$ & $\begin{array}{l}\text { Axial } \\
\text { Planes }\end{array}$ \\
\hline 7-Pin & 33 & L3T5 & 265 & A1R1 & 78 \\
\hline 91-Pin & 33 & L3T5 & 3061 & A1R1 & 78 \\
\hline
\end{tabular}

For a uniform $300 \mathrm{~K}$ temperature distribution, the axial power distribution and relative power magnitude across pins is nearly identical in the 7-pin configuration and also in the 91-pin configuration, meaning the center pin power axial distribution is representative of all pins in each configuration. This simplifies the amount of data required to be transferred to Nek5000 as the same power profile can be applied to all pins.

\section{B.5 Discussion of PROTEUS-SN vs. PROTEUS-MOCEX Results}

The PROTEUS-SN code was not used for the 7-pin configuration due to convergence issues related to the boundary conditions. However, it was used to solve a limited number of test cases for the 91-pin configuration. The code performance was much slower than PROTEUS-MOCEX, by a factor of 100-1000x the computational cost, most likely due to the reflective boundary conditions. While we assess is not practical to use the PROTEUS-SN code for this geometry, we present some results for comparison, since this is the solver within SHARP. 
Table B.5.1 displays several eigenvalue results using both codes with varying levels of mesh and angular refinement. It is well known that the SN code (based on the continuous finite element method) requires many more finite elements than the MOCEX code for similar accuracy due to the inability to capture steep spatial gradients with a coarse mesh. The results show a discrepancy of about $210 \mathrm{pcm}$ between the most refined SN and MOCEX calculations which we have not been able to resolve. It may be an inherent issue with one of the methodologies for reflected boundary conditions. Regardless, the MOCEX result is more trustworthy for this calculation due to its underlying methodology being accurate for heterogeneous calculations.

Table B.5.1 MOCEX vs. SN eigenvalue results for uniform $300 \mathrm{~K}$ temperature (91-pin)

\begin{tabular}{|c|c|c|c|}
\hline Code & $\begin{array}{l}\text { Options } \\
A=\text { Azimuthal mesh fidelity } \\
\mathrm{R}=\text { Radial mesh fidelity } \\
\mathrm{Z}=\text { Axial mesh fidelity }\end{array}$ & Eigenvalue & Computational Cost \\
\hline MOCEX & $\begin{array}{l}\text { 33g L3T5 A1R1Z2 } \\
\text { (3540 ele/plane, } 78 \text { planes) }\end{array}$ & 1.10327 & $\begin{array}{l}62 \text { core-hours } \\
(1.3 \mathrm{hr} \text { on } 48 \text { Blues cores })\end{array}$ \\
\hline MOCEX & $\begin{array}{l}\text { 33g L3T5 A2R1Z2 } \\
\text { (7080 ele/plane, } 78 \text { planes) }\end{array}$ & 1.10323 & $\begin{array}{l}139 \text { core-hours } \\
(2.9 \mathrm{hr} \text { on } 48 \text { Blues cores })\end{array}$ \\
\hline MOCEX & $\begin{array}{l}\text { 33g L3T5 A4R2Z2 } \\
\text { (18528 vert/plane, } 78 \text { planes) }\end{array}$ & 1.10324 & $\begin{array}{l}298 \text { core-hours } \\
(3.1 \mathrm{hr} \text { on } 96 \text { Blues cores })\end{array}$ \\
\hline MOCEX & $\begin{array}{l}\text { 33g L7T11 A1R1Z3 } \\
\text { (3540 ele/plane, } 90 \text { planes) }\end{array}$ & 1.10340 & $\begin{array}{l}206 \text { core-hours } \\
\text { ( } 4.3 \mathrm{hr} \text { on } 48 \text { Blues cores })\end{array}$ \\
\hline SN & $\begin{array}{l}\text { 33g L3T5 A2R1Z1 } \\
\text { (7080 ele/plane, } 55 \text { planes) }\end{array}$ & 1.09995 & $\begin{array}{l}1536 \text { core-hours } \\
\text { ( } 1.5 \mathrm{hr} \text { on } 1024 \text { Blues cores) }\end{array}$ \\
\hline SN & $\begin{array}{l}\text { 33g L3T5 A4R2Z1 } \\
\text { (18528 ele/plane. } 55 \text { planes) }\end{array}$ & 1.10090 & $\begin{array}{l}7782 \text { core-hours } \\
\text { ( } 3.8 \mathrm{hr} \text { on } 2048 \text { Blues cores) }\end{array}$ \\
\hline SN & $\begin{array}{l}\text { 33g L7T11 A2R1Z1 } \\
\text { (7080 ele/plane, } 55 \text { planes) }\end{array}$ & 1.10042 & $\begin{array}{l}8806 \text { core-hours } \\
\text { ( } 4.3 \mathrm{hr} \text { on } 2048 \text { Blues cores })\end{array}$ \\
\hline $\mathrm{SN}$ & $\begin{array}{l}\text { 33g L7T11 A2R2Z3 } \\
\text { (9264 ele/plane, } 90 \text { planes) }\end{array}$ & 1.10119 & $\begin{array}{l}109773 \text { core-hours } \\
6.7 \mathrm{hr} \text { on } 16384 \mathrm{BGQ} \text { cores }\end{array}$ \\
\hline
\end{tabular}




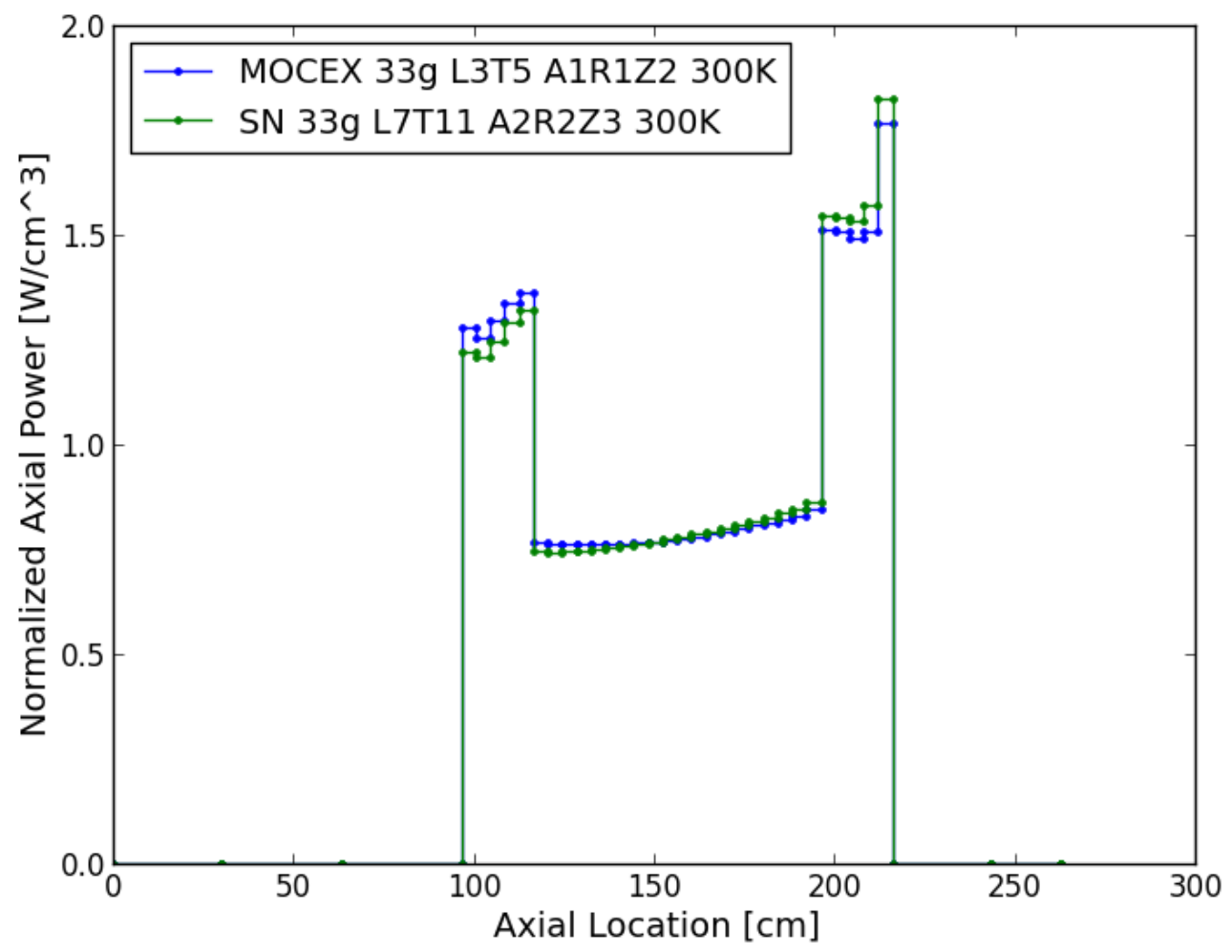

Figure B.5.1 MOCEX vs. SN axial power profile comparison for uniform $300 \mathrm{~K}$ (91-pin)

Figure B.5.1 shows the normalized axial power profiles between the most converged MOCEX and SN solutions available. There are inherent differences: the power appears to be shifted towards the top of the core in the SN solution, yielding a higher peak/average axial value (1.8256) vs the MOCEX value (1.7654). This is a difference of $+3.4 \%$. The SN solution should be simulated in Nek5000 to determine the sensitivity of Nek5000 on this difference.

In summary, while the PROTEUS-SN code can be used to solve the 91-pin configuration, it is not recommended at this point due to unexplained differences from the PROTEUS-MOCEX simulation which we believe to be more accurate for this geometry. It is also much more computationally expensive for this problem. Nonetheless, if the discrepancy cannot be resolved, the SN power profile should be simulated in Nek5000 to determine the effect on the hot channel factor, if any.

\section{B.6 Discussion of Initial Temperature Condition}

The previous neutronics calculations assumed a uniform $300 \mathrm{~K}$ temperature (room temperature), since there is no temperature interpolation capability in PROTEUS-MOCEX, and the PROTEUSSN temperature interpolation capability was limited to SHARP coupled problems (called from SHARP driver). This is valid because the power shape does not usually have a strong dependence on temperature. Since the average core temperature is actually closer to $800 \mathrm{~K}$, a uniform $800 \mathrm{~K}$ simulation was tested to assess any differences in the axial power profile. 
Between the uniform $300 \mathrm{~K}$ and $800 \mathrm{~K}$ temperature simulations, the 7-pin $800 \mathrm{~K}$ simulation eigenvalue was lower by $653 \mathrm{pcm}$ as shown in Table B.6.1. However, the normalized power profiles for $300 \mathrm{~K}$ and $800 \mathrm{~K}$, shown in Figure B.6.1, did not exhibit significant difference in terms of shape or magnitude. The peak axial power differed by only $0.6 \%$ in the two cases. Additionally, the fuel pins in each ring did not show significant differences in their reported power.

Table B.6.1 MOCEX eigenvalue results at two uniform temperatures (7-pin)

\begin{tabular}{|l|l|l|l|l|l|l|l|}
\hline $\begin{array}{l}\text { Energy } \\
\text { Group }\end{array}$ & Eigenvalue & $\begin{array}{l}\text { Peak / } \\
\text { Average } \\
\text { Power }\end{array}$ & Temperature & $\begin{array}{l}\text { Angular } \\
\text { Cubature }\end{array}$ & $\begin{array}{l}\text { Mesh } \\
\text { Elements } \\
(2 \mathrm{D})\end{array}$ & $\begin{array}{l}\text { Mesh } \\
\text { Refinement }\end{array}$ & Planes \\
\hline 70 & 1.12649 & 1.9364 & $300 \mathrm{~K}$ & L3T5 & 265 & A1R1 & 78 \\
\hline 70 & 1.11996 & 1.9250 & $800 \mathrm{~K}$ & L3T5 & 265 & A1R1 & 78 \\
\hline
\end{tabular}

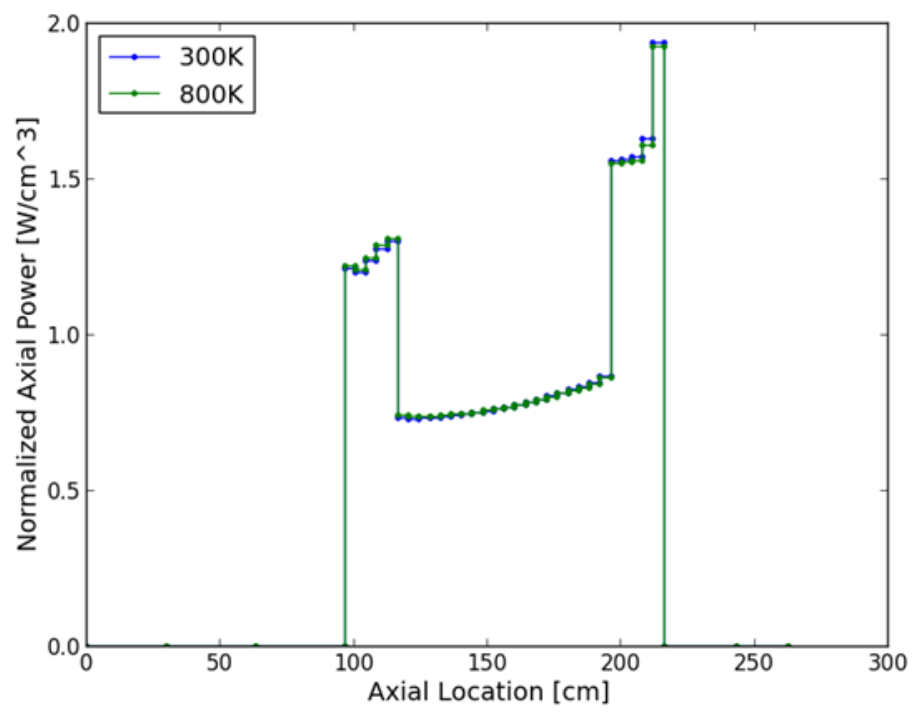

Figure B.6.1 MOCEX comparison of power profile at 300K and $800 \mathrm{~K}$ (7-pin).

The 91-pin $800 \mathrm{~K}$ simulation eigenvalue was 874 pcm lower than the $300 \mathrm{~K}$ value, shown in Table B.6.2. The peak/average axial pin powers are listed in Table B.6.3 by ring number. One can see that the higher $800 \mathrm{~K}$ uniform temperature elevates the peak/average power at the top of the active core by

Table B.6.2 MOCEX eigenvalue results at two uniform temperatures (91-pin)

\begin{tabular}{|l|l|l|l|l|l|l|}
\hline $\begin{array}{l}\text { Energy } \\
\text { Group }\end{array}$ & Eigenvalue & Temperature & $\begin{array}{l}\text { Angular } \\
\text { Cubature }\end{array}$ & $\begin{array}{l}\text { Mesh } \\
\text { Elements } \\
(2 \mathrm{D})\end{array}$ & $\begin{array}{l}\text { Mesh } \\
\text { Refinement }\end{array}$ & Planes \\
\hline
\end{tabular}




\begin{tabular}{|l|l|l|l|l|l|l|}
\hline 33 & 1.10327 & $300 \mathrm{~K}$ & L3T5 & 3061 & A1R1 & 78 \\
\hline 33 & 1.09453 & $800 \mathrm{~K}$ & L3T5 & 3061 & A1R1 & 78 \\
\hline
\end{tabular}

Table B.6.3 MOCEX peak/average pin power in each ring at two uniform temperatures (91pin)

\begin{tabular}{|l|l|l|l|}
\hline Ring & $\begin{array}{l}\text { Peak/Average } \\
\text { Axial Power } \\
\text { per Pin } \\
(300 \mathrm{~K})\end{array}$ & $\begin{array}{l}\text { Peak/Average } \\
\text { Axial Power } \\
\text { per Pin } \\
(800 \mathrm{~K})\end{array}$ & $\begin{array}{l}\text { \% Increase in } \\
\text { peak/average } \\
\text { pin power at } \\
800 \mathrm{~K}\end{array}$ \\
\hline 1 (center) & 1.7654 & 1.7972 & $+1.8 \%$ \\
\hline 2 & 1.7651 & 1.7962 & $+1.8 \%$ \\
\hline 3 & 1.7649 & 1.8009 & $+2.0 \%$ \\
\hline 4 & 1.7652 & 1.8077 & $+2.4 \%$ \\
\hline 5 & 1.7673 & 1.8225 & $+3.1 \%$ \\
\hline 6 (next to duct) & 1.7810 & 1.8726 & $+5.1 \%$ \\
\hline
\end{tabular}

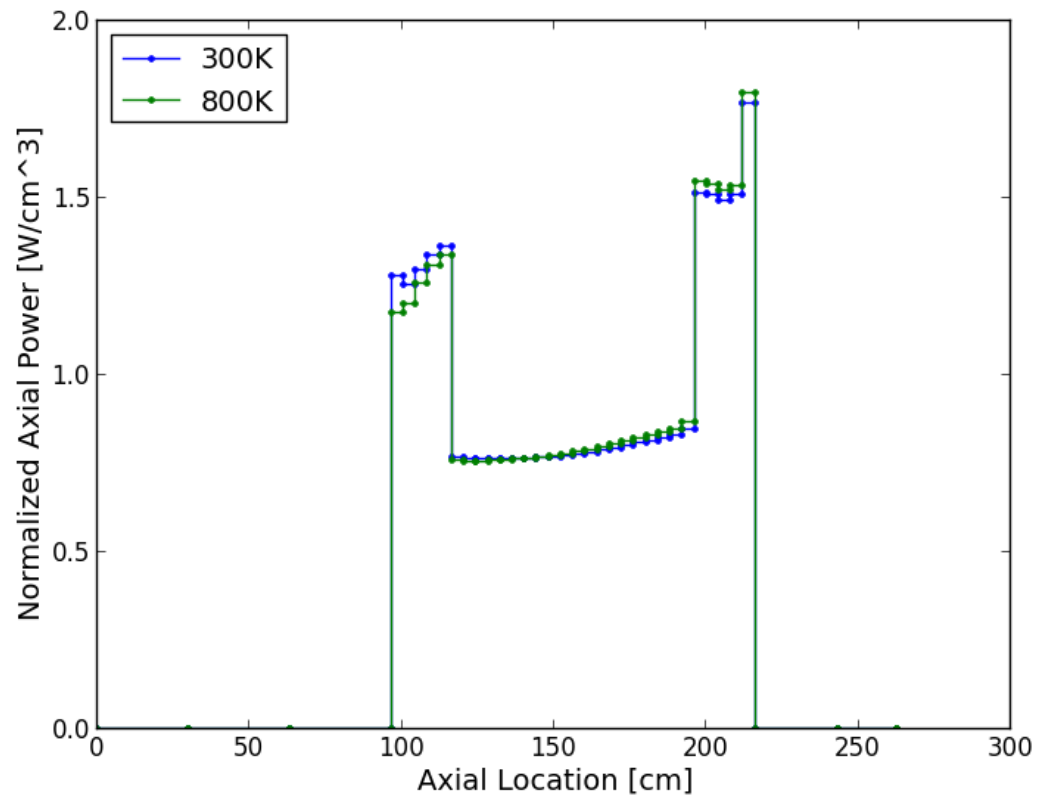

Figure B.6.2 MOCEX comparison of power profile at $300 \mathrm{~K}$ and $800 \mathrm{~K}$ (91-pin).

However, uniform temperature profiles are not representative of the true temperature condition in the reactor which varies along the axial dimension due to the power shape. The temperature range in the fuel, cladding, and sodium coolant were estimated by quick STAR-CCM+ calculations and shown in Table B.6.3.

Table B.6.3 Estimated temperature ranges for 7-pin and 91-pin configurations 


\begin{tabular}{|l|c|c|}
\hline \multicolumn{1}{|c|}{ Zone } & $\begin{array}{c}\text { 7-Pin Temperature } \\
\text { Range }\end{array}$ & $\begin{array}{c}\text { 91-Pin Temperature } \\
\text { Range }\end{array}$ \\
\hline Fuel & $718-854 \mathrm{~K}$ & $668-948 \mathrm{~K}$ \\
\hline Cladding & $672-808 \mathrm{~K}$ & $668-863 \mathrm{~K}$ \\
\hline Sodium Coolant & $668-784 \mathrm{~K}$ & $668-873 \mathrm{~K}$ \\
\hline
\end{tabular}

In order to use a non-uniform temperature profile as the initial condition, code development was needed. First, automatic temperature interpolation was enabled in standalone PROTEUS-SN. This feature was previously enabled from the SHARP driver using Nek5000 temperature feedback to drive the cross section interpolation, so enabling temperature feedback in initial condition neutronics calculations required only modest code changes due to the existing infrastructure. For PROTEUS-MOCEX, a script was developed to define linear combinations of materials at different temperatures to simulate temperature interpolation via the input file.

The lower temperature bound in Table B.6.3 was assigned to the bottom of the active fuel zone, and the upper temperature bound was assigned to the top of the active fuel zone. The middle of the active fuel zone was assigned temperatures based on linear interpolation of the lower and upper bounds. Materials above and below the active core were assigned a constant value according to the lower or upper range. Using these temperatures for an initial condition in neutronics should yield a good estimate of the coupled SHARP result that iterates back and forth between neutronics and $\mathrm{T} / \mathrm{H}$.

The results of the temperature gradient study are shown in the following table and figures. Applying the temperature gradient impacted the axial power profile by suppressing the power in the upper-most zones of the active fuel, lowering the peak / average power of the axial power profile. This behavior was observed in both the MOCEX and SN, although the impact was greater in the MOCEX calculations. The difference between the SN and MOCEX results is relatively significant, as mentioned in the preceding section. For the temperature gradient calculations, the power peaking is 1.7045 in MOCEX vs 1.7926 in SN. This could cause a large effect on the thermal hydraulics calculation of temperature. This will be investigated in FY18.

Table B.6.4 MOCEX and SN peak / average axial power results for temperature profile initial condition (91 pin)

\begin{tabular}{|l|l|l|l|}
\hline Code & Temperature & Discretization & $\begin{array}{l}\text { Peak/Avg Axial } \\
\text { Power }\end{array}$ \\
\hline MOCEX & Uniform 300 K & 33g L3T5 R1A1 78 planes & 1.7654 \\
\hline MOCEX & Temp. Profile & 33g L3T5 R1A1 78 planes & 1.7045 \\
\hline
\end{tabular}




\begin{tabular}{|l|l|l|l|}
\hline SN & Uniform 300 K & 33g L7T11 R2A2 90 planes & 1.8256 \\
\hline SN & Temp. Profile & 33g L7T11 R2A2 90 planes & 1.7926 \\
\hline
\end{tabular}

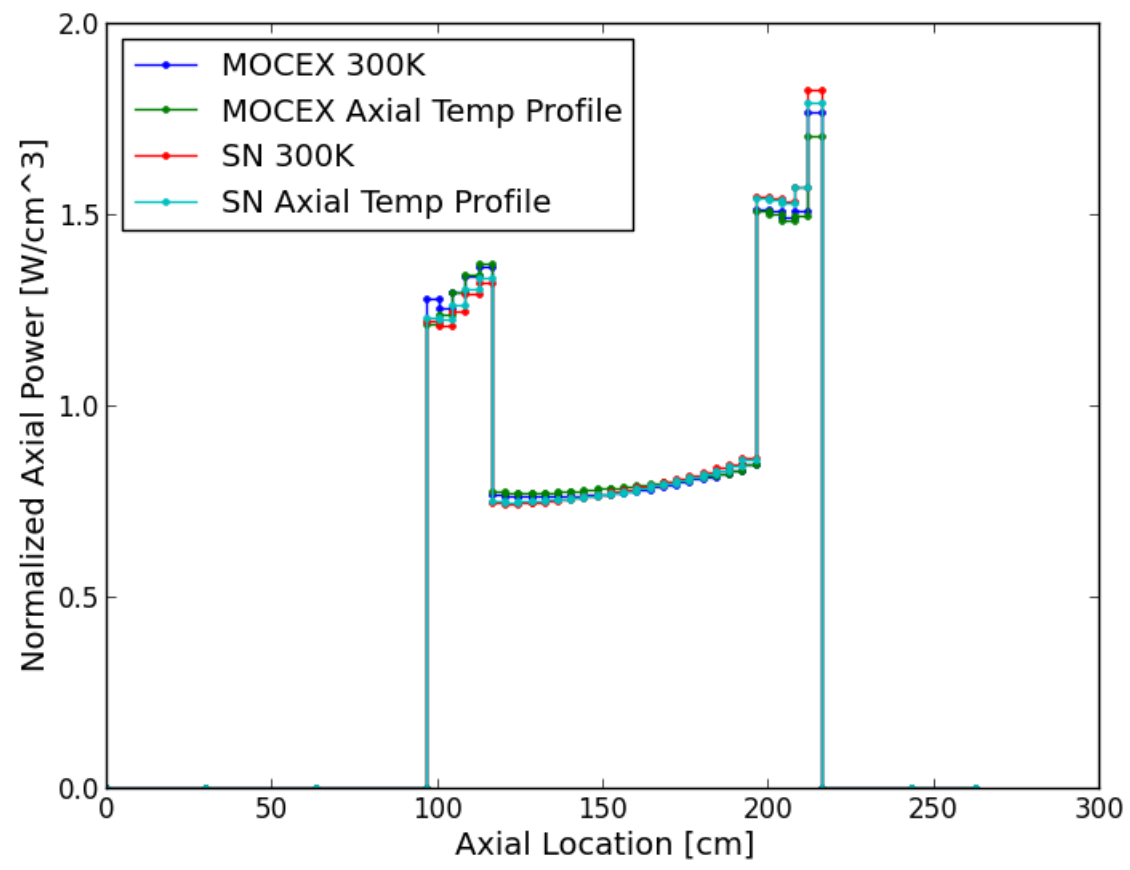

Figure B.6.3 MOCEX and SN axial power distributions for different temperature initial conditions (91-pin)

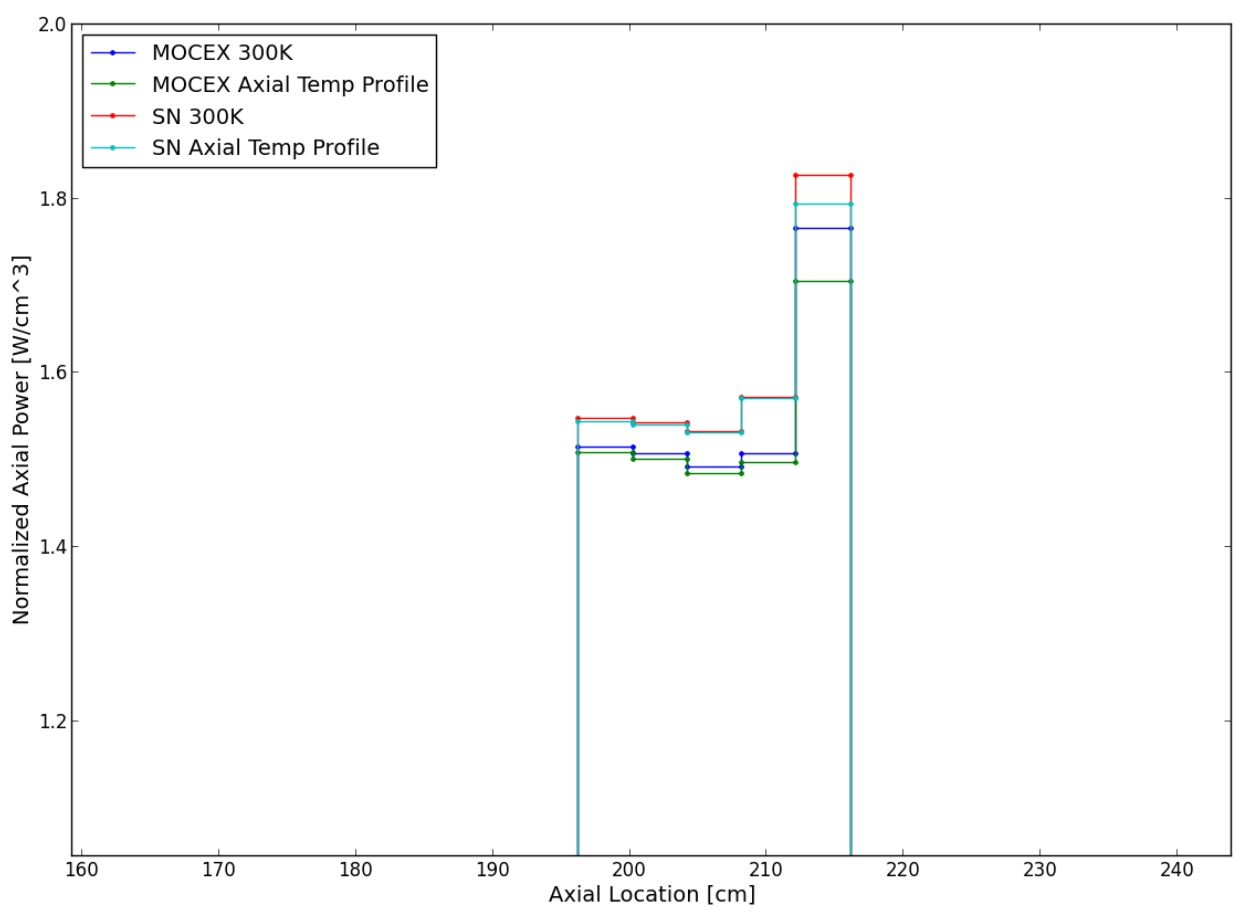


Figure B.6.4 MOCEX and SN axial power distributions for different temperature initial conditions, zoomed in at upper $\mathbf{1 8 . 0 \%}$ enriched core region to show detail (91-pin) 


\section{APPENDIX C. NEK5000 SENSITIVITY STUDY}

\section{C.1. Primary STAR-CCM+ calculations}

Nek5000 is generally computationally expensive due to its high fidelity features and requires more manpower resources than other commercial CFD tools. In the early stages of investigation, it is necessary to perform some preliminary calculations to obtain basic information on the problem such as the temperature range, the sensitivity to the mesh and the model. We use STAR-CCM+ for these preliminary calculation and sensitivity studies at the early stages due its relatively low computational requirements.

STAR-CCM+ is a commercial CFD code which is developed by CD-Adapco (Computational Dynamics-Analysis \& Design Application Company Ltd), a multinational computer software company that authors and distributes applications used for computer-aided engineering. STAR$\mathrm{CCM}+$ included the world's first commercially available polyhedral meshing algorithm. The use of a polyhedral mesh has proven to be more accurate for fluid-flow problems than a hexahedral or tetrahedral mesh of a similar size (number of cells), but is considerably more difficult to create. Due to the complexity of the geometric feature of wire wrap rod bundle, meshing the model is very challenging. As so, the automatic polyhedral meshing technology built in STAR-CCM+ is very useful. The polyhedral mesh snapshot for wire wrap rod bundle is shown in Figure C.1.1. The prismatic cell layers generated can help capture the boundary layer, turbulence effects, and heat transfer near the wall boundaries.
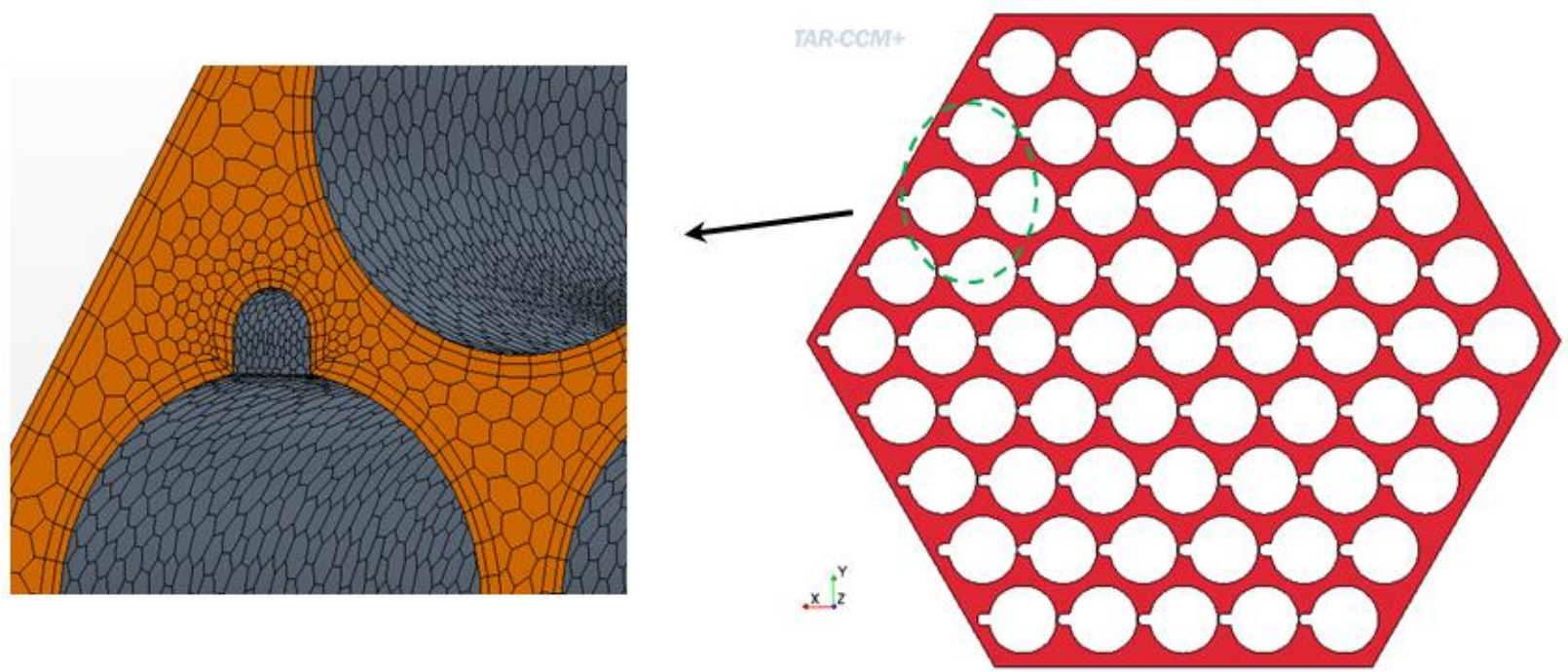

Figure C.1.1 Snapshot of polyhedral mesh for wire wrap rod bundle

The objects of the primary calculation with the commercial CFD code STAR-CCM+ are as follow:

O Justify the simplification: The AFR drive assembly has 91 fuel rods. The height of the active core is about 5 wire wrap pitches. If we choose to perform the calculation for the 
wire wrap rod bundle of full length (5 wire wrap pitches), the cell number of the simulation could easily reach 1 2 billion. Those simulations become either too costly or unnecessary. Preliminary calculations can help us to find and justify reasonable simplifications which have a tradeoff between fidelity and computational cost.

- Provide references and initial conditions: Preliminary simulations can provide the basic information on velocity and temperature distribution within the assembly. The information can be applied to initial conditions preparation, mesh generation and code-to-code comparison for further Nek5000 calculation.

In order to achieve the goals mentioned above, several sensitivity studies and preliminary calculations with STAR-CCM+ are performed as listed:

- Full length vs 1 wire wrap pitch: The wire wrap rod bundle shows the periodic geometric feature. It is reasonable to assume that the flow behavior is periodic so that we can only model the domain of 1 wire wrap pitch to capture the flow behavior in the interested area.

- Power distribution of BOC vs uniform power distribution: The axial power distribution can bring in some error if we only use 1 wire wrap pitch domain. The calculation can help us to quantify the error.

- Bare rod bundle+ Momentum source term vs Wire wrap rod bundle: The wire wrap rod bundle has more complex geometry than bare rod bundle. We can introduce the wire effect in bare rod bundle geometry by implanting some momentum source term. The calculation can test the flexibility of this approach.

- Bare rod bundle vs Wire wrap rod bundle: The wire wrap rod bundle is usually simplified as bare rod bundle because that can reduce the difficulty of meshing and the computational cost by an order of magnitude. The calculation can estimate the effect of the wire.

- With Conjugate Heat Transfer vs Without Conjugate Heat Transfer: The effect of the heat transfer between the fluid domain and the solid domain are assessed through the calculation.

- Perturbed cladding thickness: The calculation can help us understand the effect of the cladding thickness on the Hot Channel Factor which will be further modeled in Nek5000.

Some verification studies on mesh and turbulence model are performed to make sure that the model can be properly used for the analysis. Based on the verification studies shown in Figure C.1.2, the mesh cell size of $1 \mathrm{~mm}$ (corresponding to 2.5 million cell number since the cell number will be different for different domain size) with aspect ratio 1:4 and realizable k- $\varepsilon$ turbulence model are chosen to be the practical combination. 


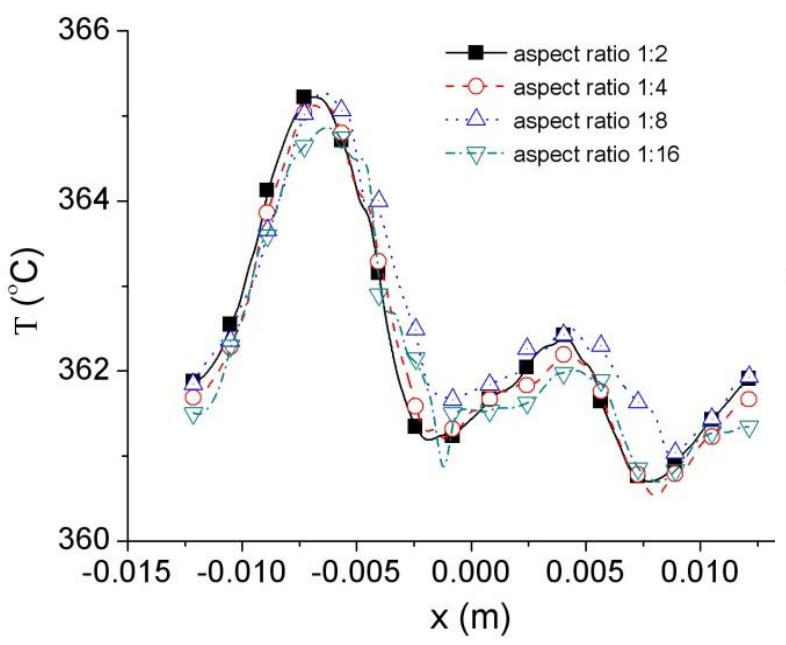

a) Mesh Aspect Ratio

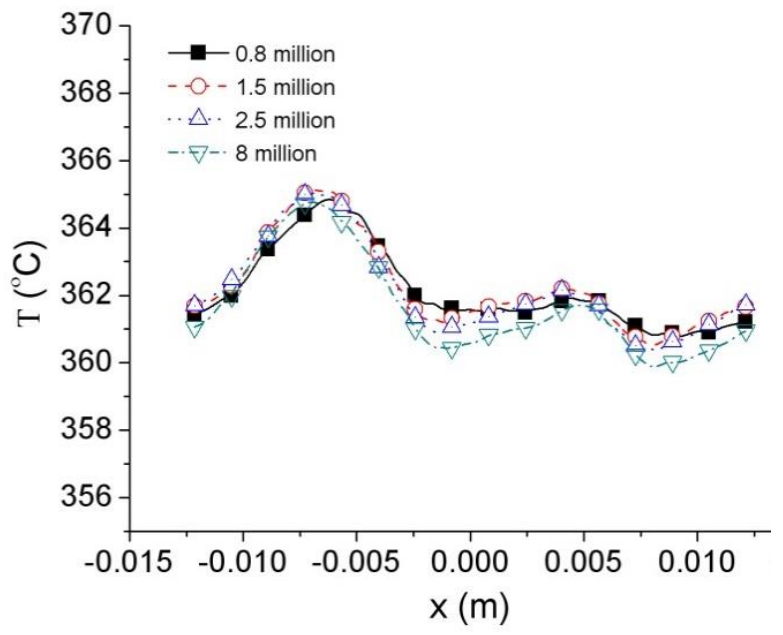

b) Mesh cell number

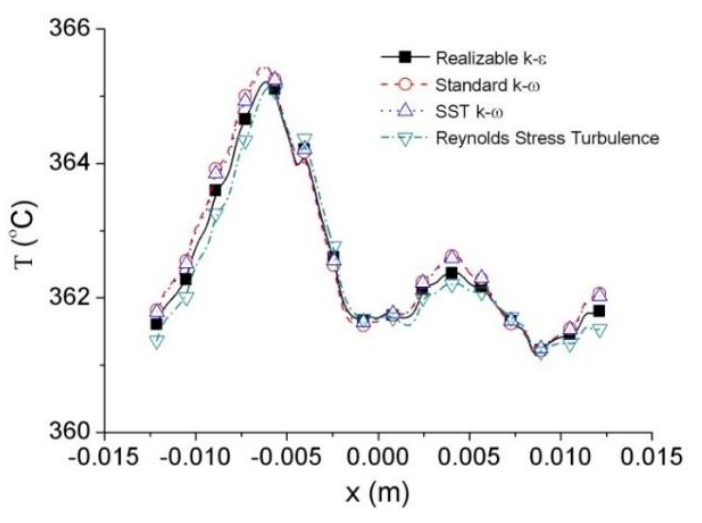

c) Turbulence Model

\section{Figure C.1.2 Verifications with STAR-CCM+}

A full length wire wrap rod bundle with conjugate hear transfer is modeled for reference. The velocity inlet and pressure outlet are adopted as the boundary conditions for the full length domain (Figure C.1.3). The total cell number reaches 13,794,629. The mesh structure is shown in Figure C.1.4. The cell size in the solid domain is relatively larger than that in the fluid domain since only heat conduction is solved in the solid domain. The temperature distributions at outlet shown in Figure C.1.5 indicate that in the center region the fluid is always hotter which leads to hotter cladding and fuel temperature. Therefore, the hot spots always appear in the center region of last pitch where the blue frame is in Figure C.1.3. Since the area of interest is only within the last pitch of the domain, a domain of 1 wire wrap pitch (Figure C.1.3) with periodic boundary condition can be adopted for predicting the flow behavior within the interested area. 

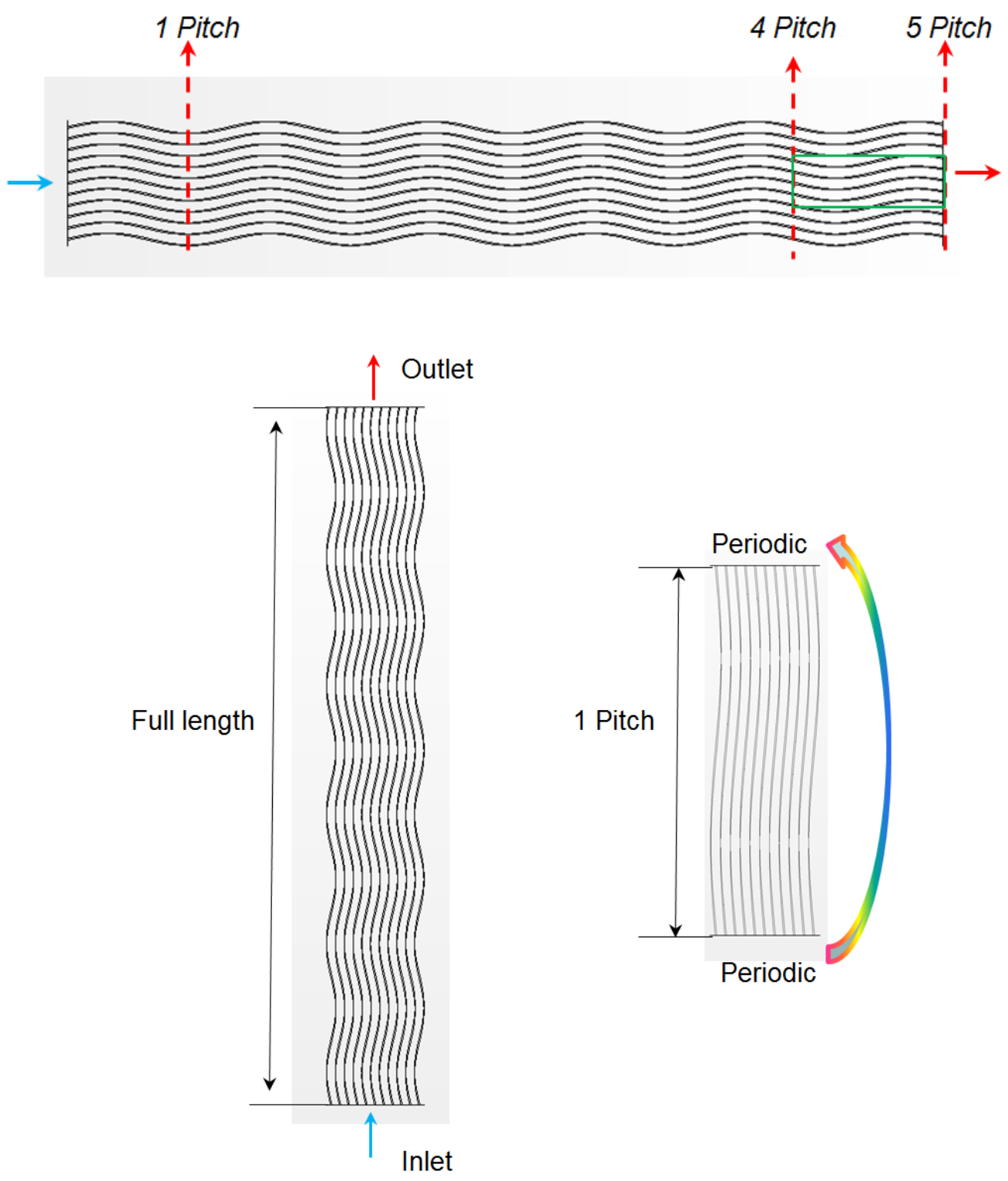

Figure C.1.3 Boundary conditions for full length domain and 1wire wrap pitch domain 

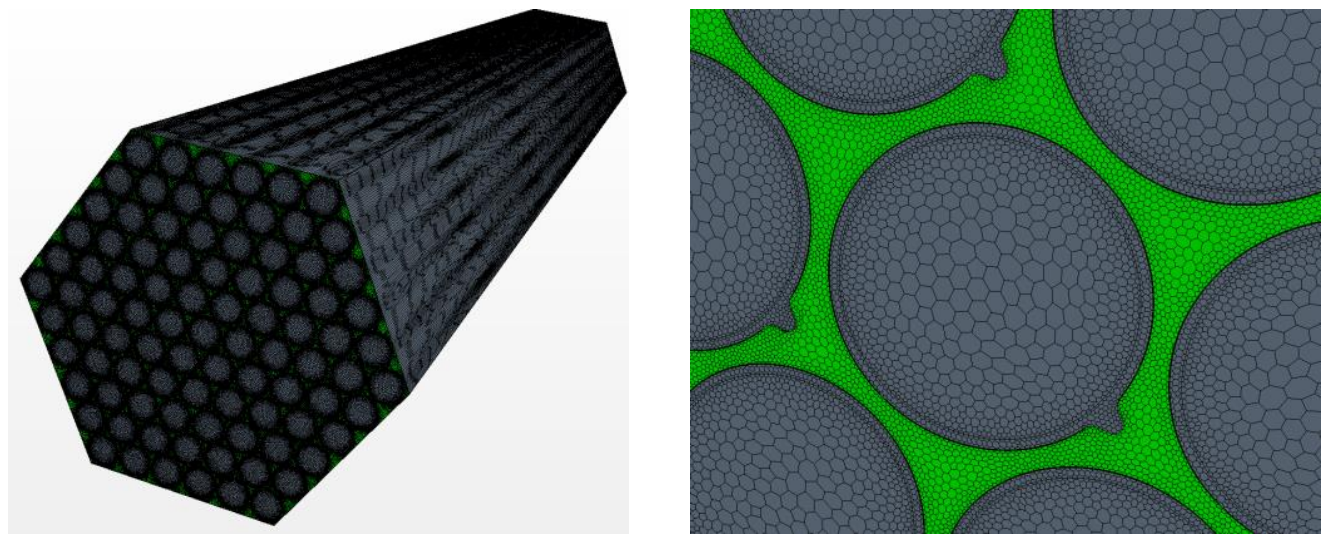

Figure C.1.4 Mesh structure for wire wrap rod bundle with cladding and fuel
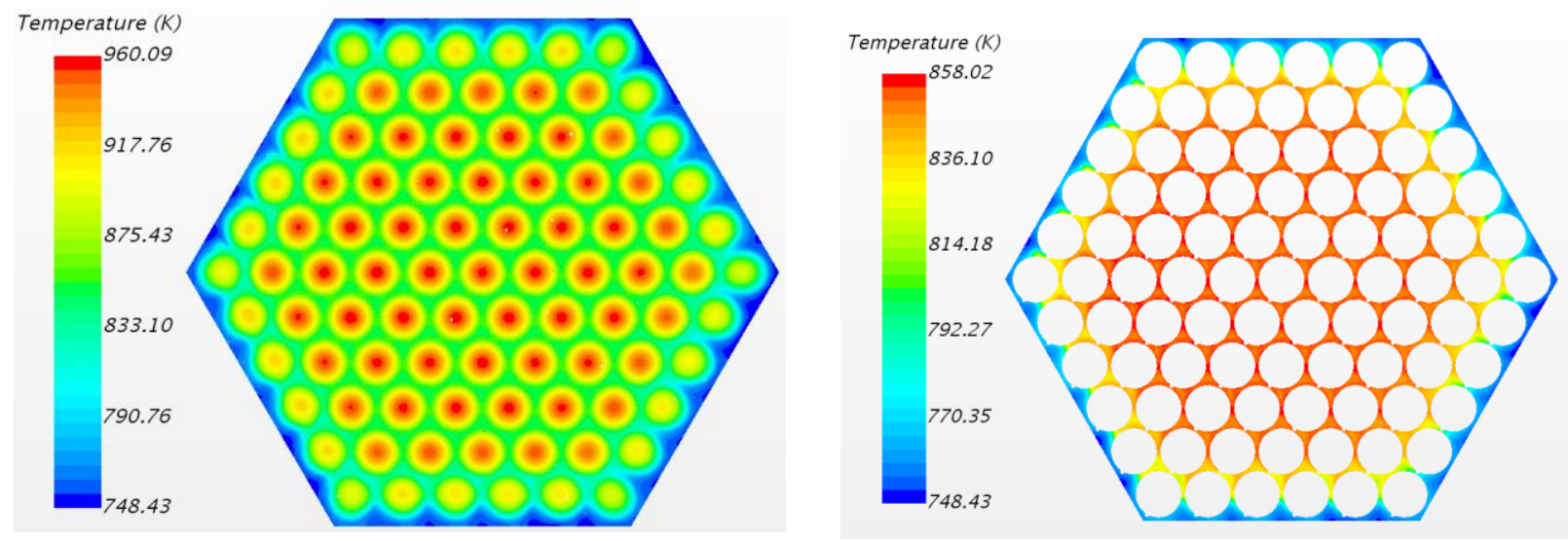

a) ALL

b) Fluid
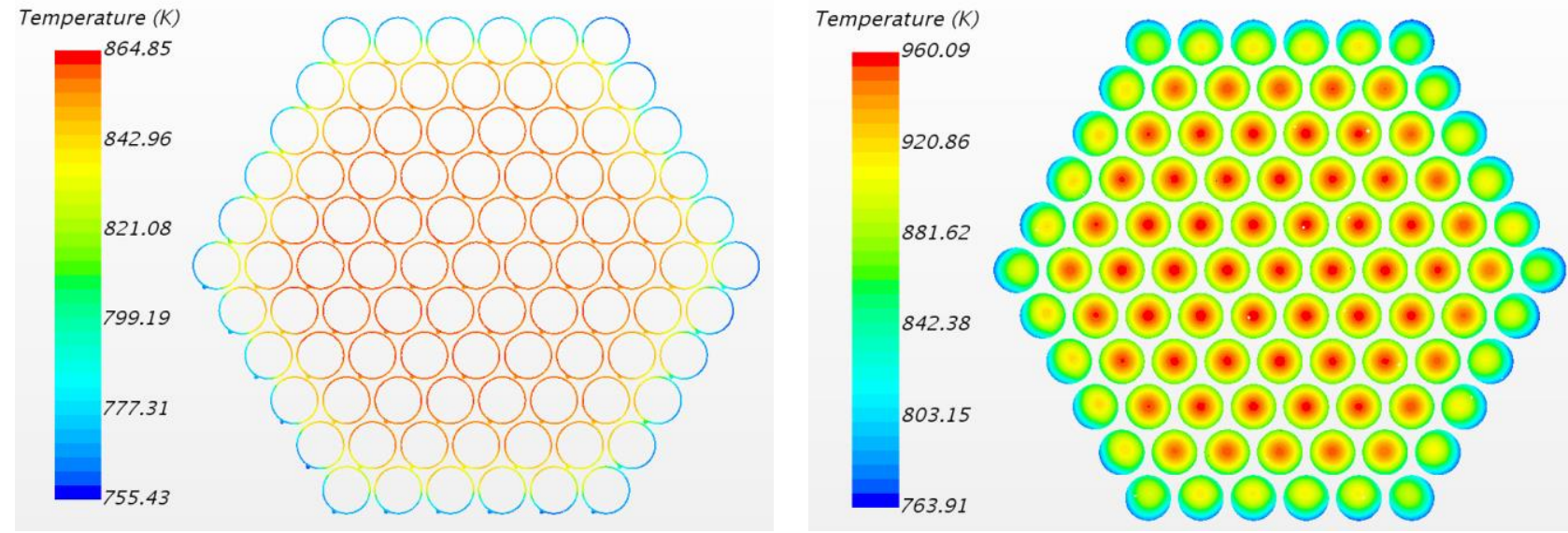

c) Cladding

d) Fuel

Figure C.1.5 Outlet temperature distributions of full length 91-pin wire wrap rod bundle with conjugate heat transfer 
For convenience, in this report "Full length" represents the domain of 5 wire wrap pitch with velocity inlet and pressure outlet boundary condition and " 1 pitch" represents the domain of 1 wire wrap pitch with periodic boundary condition in the stream-wise direction. Figure C.1.6 shows the comparison of outlet velocity distribution between Full length and 1 pitch. The contours look almost identical with only $8.9 \times 10^{-3} \mathrm{~m} / \mathrm{s}$ maximum velocity deviation. The qualitative comparison shows a global agreement of velocity distribution between Full length and 1pitch. The local velocity distribution comparison is also very necessary. Figure C.1.7 shows the detailed velocity distribution on the line between two rods for Full Length and 1 Pitch. The good agreements indicate that the domain of 1 wire wrap pitch with periodic boundary condition in the stream-wise direction can significantly simplify the model and give the same velocity prediction as the full length model can.

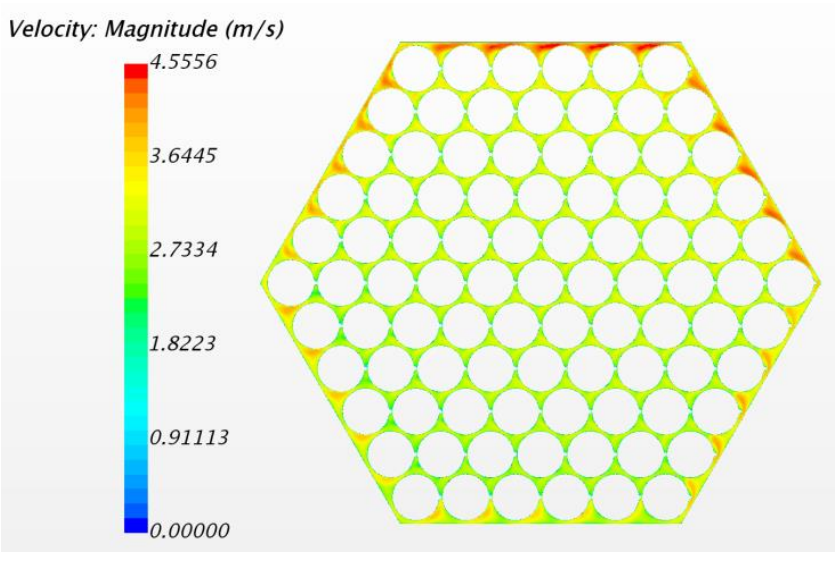

(a) Full length

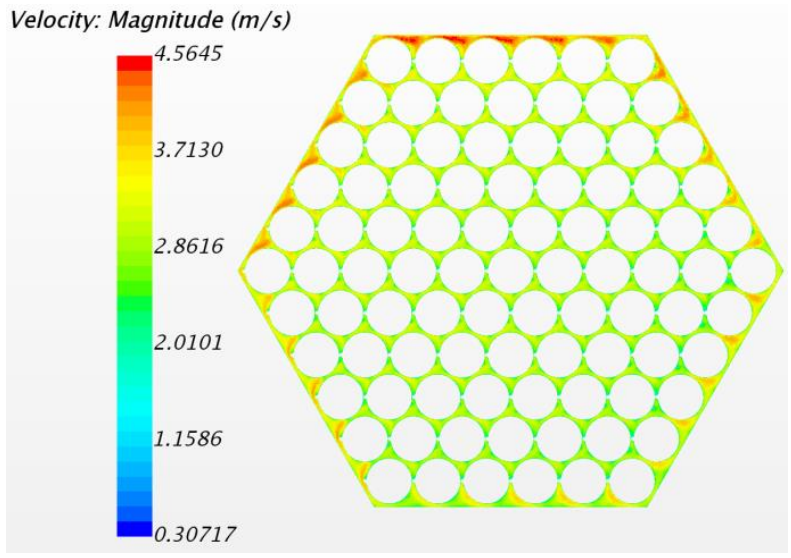

(b) 1 pitch

Figure C.1.6. Outlet velocity contour for full length and 1 pitch

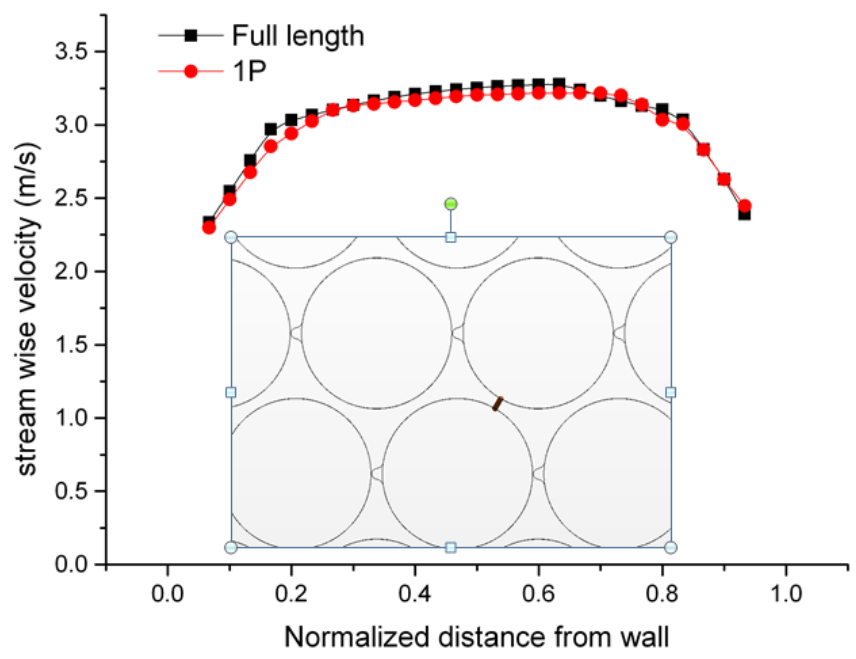

Figure C.1.7 Detailed velocity distribution on the line between rods for full length and 1 pitch 


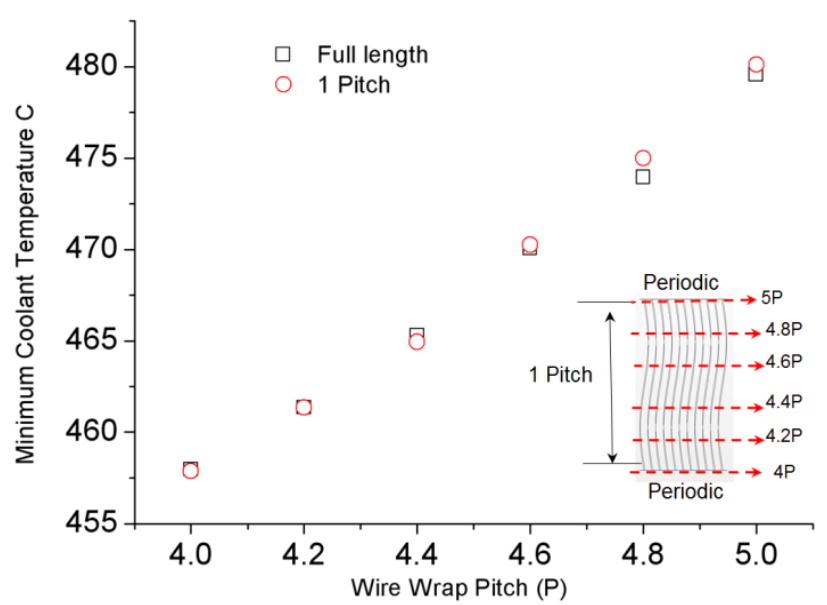

a) Minimum coolant Temperature

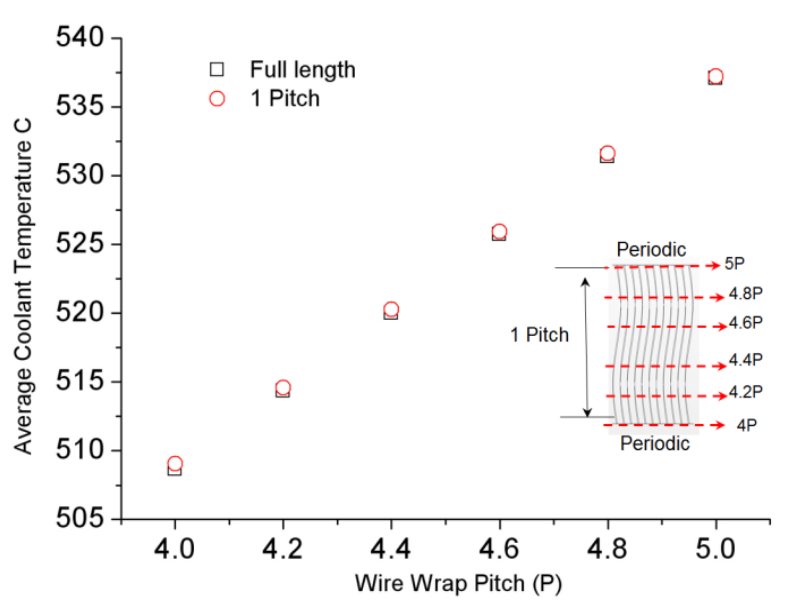

b) average coolant Temperature

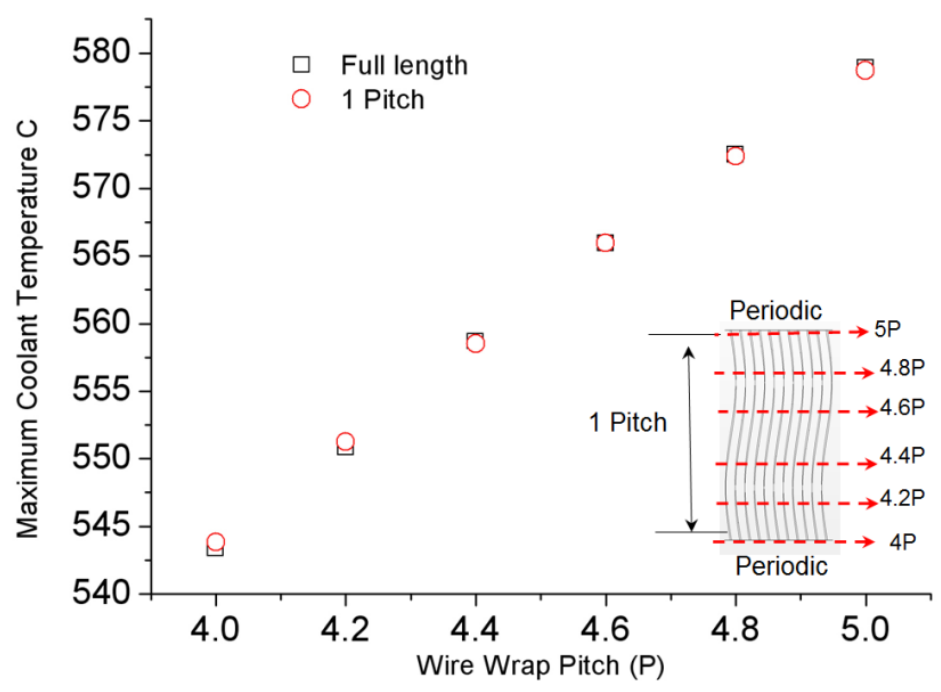

c) Maximum coolant Temperature

Figure C.1.8 Minimum, average and maximum coolant temperature comparison on each elevation between full length and 1 pitch

Temperature prediction is very critical in $\mathrm{HCF}$ calculations. Figure C.1.8 compares the minimum, average and maximum coolant temperature on elevation $4 \mathrm{P}, 4.2 \mathrm{P}, 4.4 \mathrm{P}, 4.6 \mathrm{P}, 4.8 \mathrm{P}$ and 5P between Full length and 1 pitch. All the data are almost identical, further justifying the use of the 1 pitch model with periodic boundary conditions to save computational resources.

The axial power distribution in a nuclear reactor is not uniform. Figure C.1.9 shows the normalized axial power distributions in AFR-100 [Kim 2012]. The axial power distribution of the AFR-100 is different from a chopped cosine shape, which is typical power shape in a sodium cooled fast reactor. The Begin of Cycle (BOC) power peaks at the core bottom because of the high uranium 
enrichment and lower reflector. However, the high neutron leakage through the upper structure decreases the power at the core top although the enrichment is the same as the core bottom. The power skews to the core central zone as the burnup increases because the burning zone moves into the core center. As a result, the axial power shape becomes a chopped cosine shape at End of Cycle (EOC). However, it could bring in some error if the power distribution is not uniform in CFD model. As shown in Figure C.1.10, no influence on maximum coolant temperature prediction is observed by applying BOC axial power distribution. The report will use BOC as the axial power distribution for the latter primary calculation.

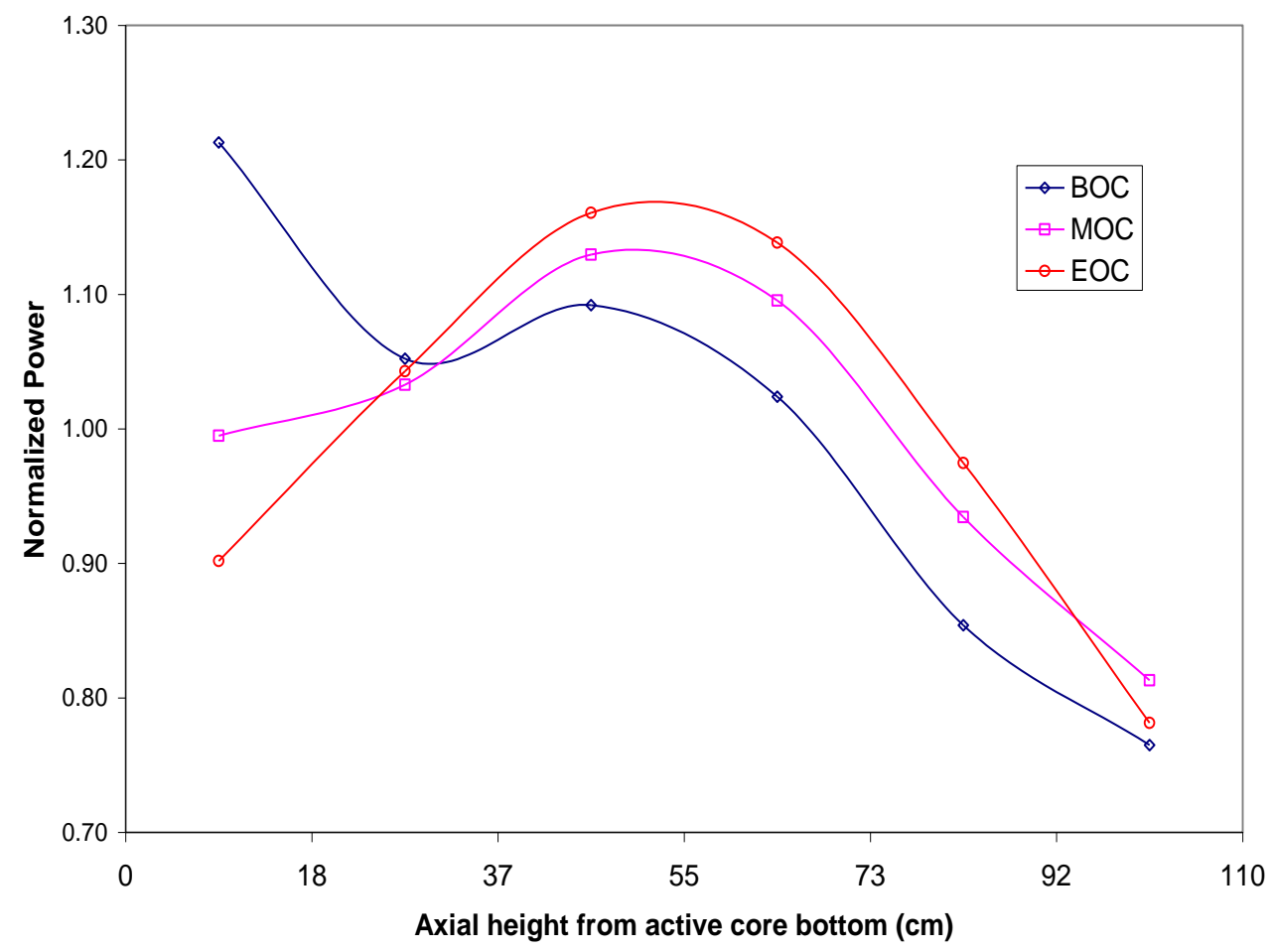

Figure C.1.9 Normalized axial power distribution for BOC, MOC and EOC of AFR-100

The wire wrap can introduce strong lateral mixing between sub-channels, which cannot be captured if we simplify the model to bare rod bundle. Figure C.1.11 shows the lateral velocity distribution in both wire wrap and bare rod bundle. The maximum lateral velocity in wire wrap rod bundle can reach about $50 \%$ of the averaged stream wise velocity while that in bare rod bundle can only reach $2 \%$ in RANS simulation. In order to improve the hydraulic modeling of wire-wrap spacers in a rod bundle, Argonne developed a three-dimensional momentum source model (MSM) [Hu 2013] to model the anisotropic flow without the need to resolve the geometric details of the wire-wraps. Computing resources can be saved with the MSM since it can be used on a much coarser computational mesh. The lateral velocity distribution predicted by bare rod bundle with momentum source is also shown in Figure C.1.11. As can be seen, the magnitude of the lateral velocity is improved by the MSM model although it still fails to capture some detailed velocity 
distribution to some extent. Figure C.1.12 shows the hotpots distribution on each elevation at last pitch of the assembly. The hot spots are defined as the locations where the temperature is within the range $99 \% \sim 100 \%$ of the maximum temperature. It reconfirms that the hottest area should appear in the center of the assembly. In addition, it is easy to see some asymmetric distribution feature of hot spots which is due to the wire wrap effect.

However, it is very significant to estimate how much effect the wire wrap can bring in on predicting the $\mathrm{HCF}$, in other word, the maximum temperature. Although strong lateral mixing is introduced by the wire wrap, the temperature distribution does not necessarily respond to that effect intensively because the radial power distribution is identical which leads to similar average subchannel temperature. Table C.1.1 lists the maximum coolant temperature with different model. The bare rod bundle model has about $7^{\circ} \mathrm{C}$ deviations of maximum coolant temperature prediction from wire wrap rod bundle model. It indicates the lateral velocity does enhance the mixing between the sub-channels which reduce the maximum coolant temperature. The MSM model only has $1.237^{\circ} \mathrm{C}$ deviations since it captures the lateral velocity better.

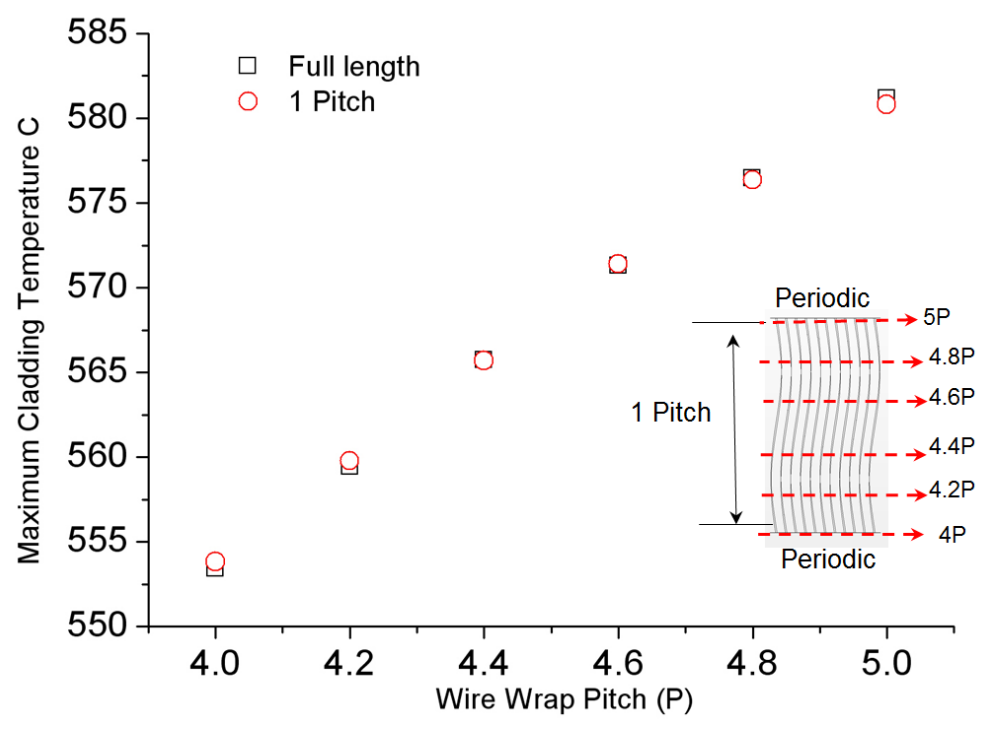

Figure C.1.10 Maximum coolant temperature comparison at different elevations for full length and 1 Pitch models with BOC axial power distribution 


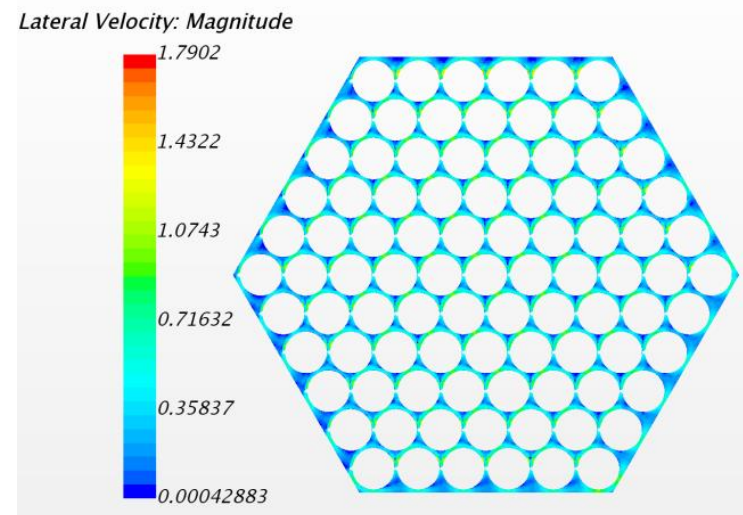

a) Wire wrap rod bundle

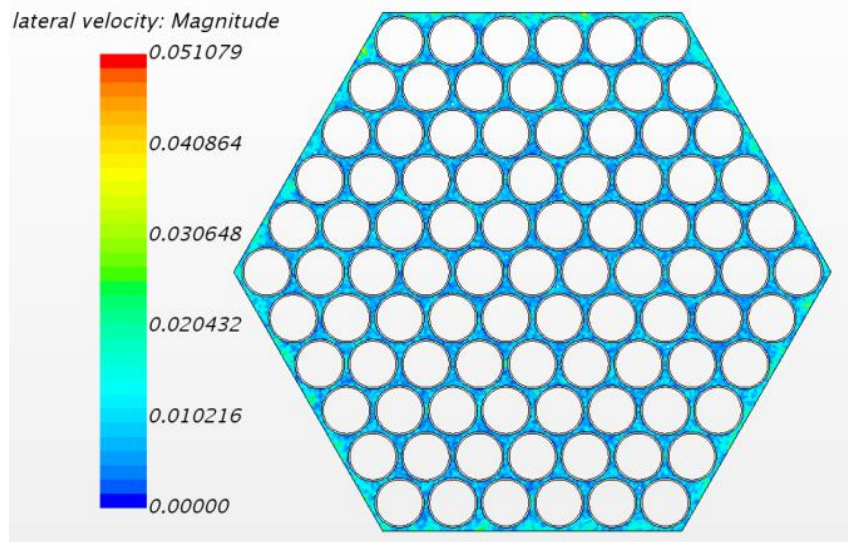

b) Bare rod bundle

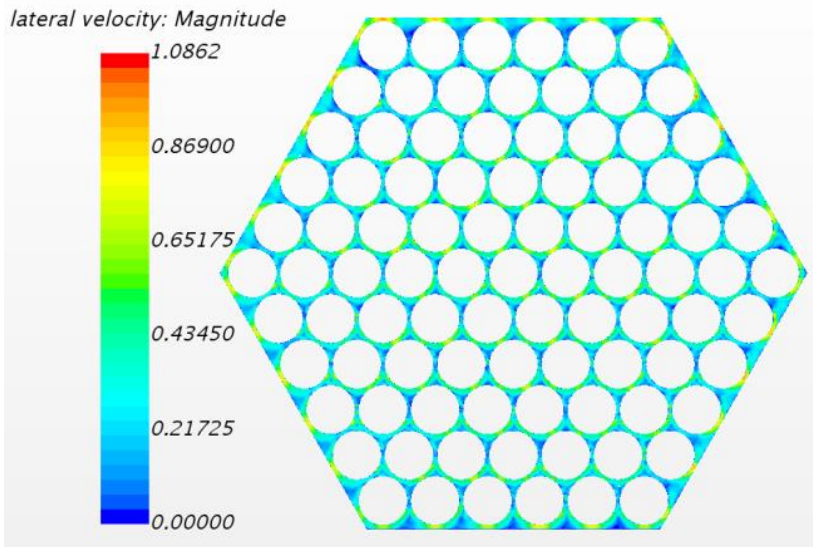

c) Bare rod bundle + Momentum source

Figure C.1.11 Lateral velocity distribution 


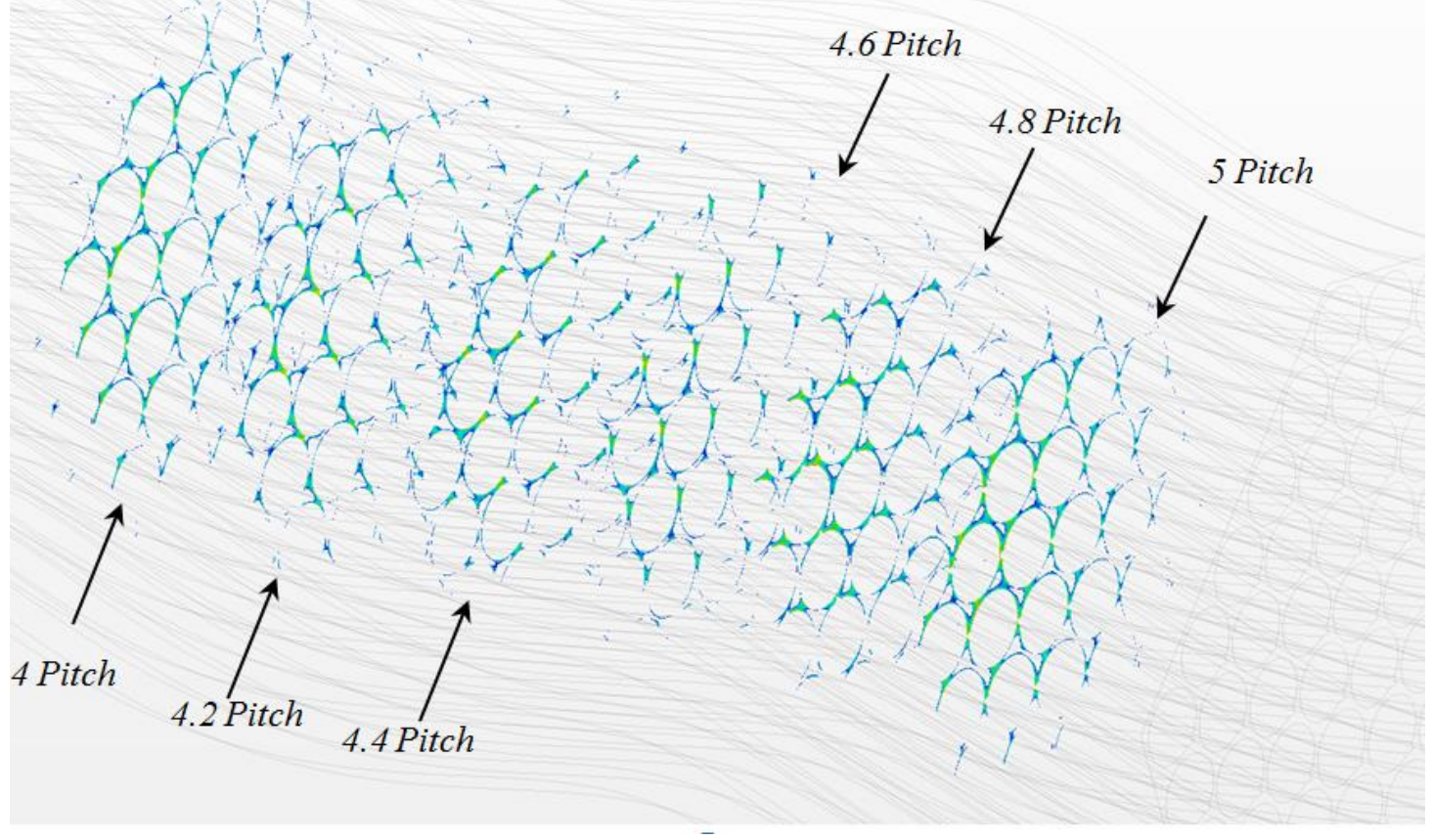

a) hot spots on each elevations

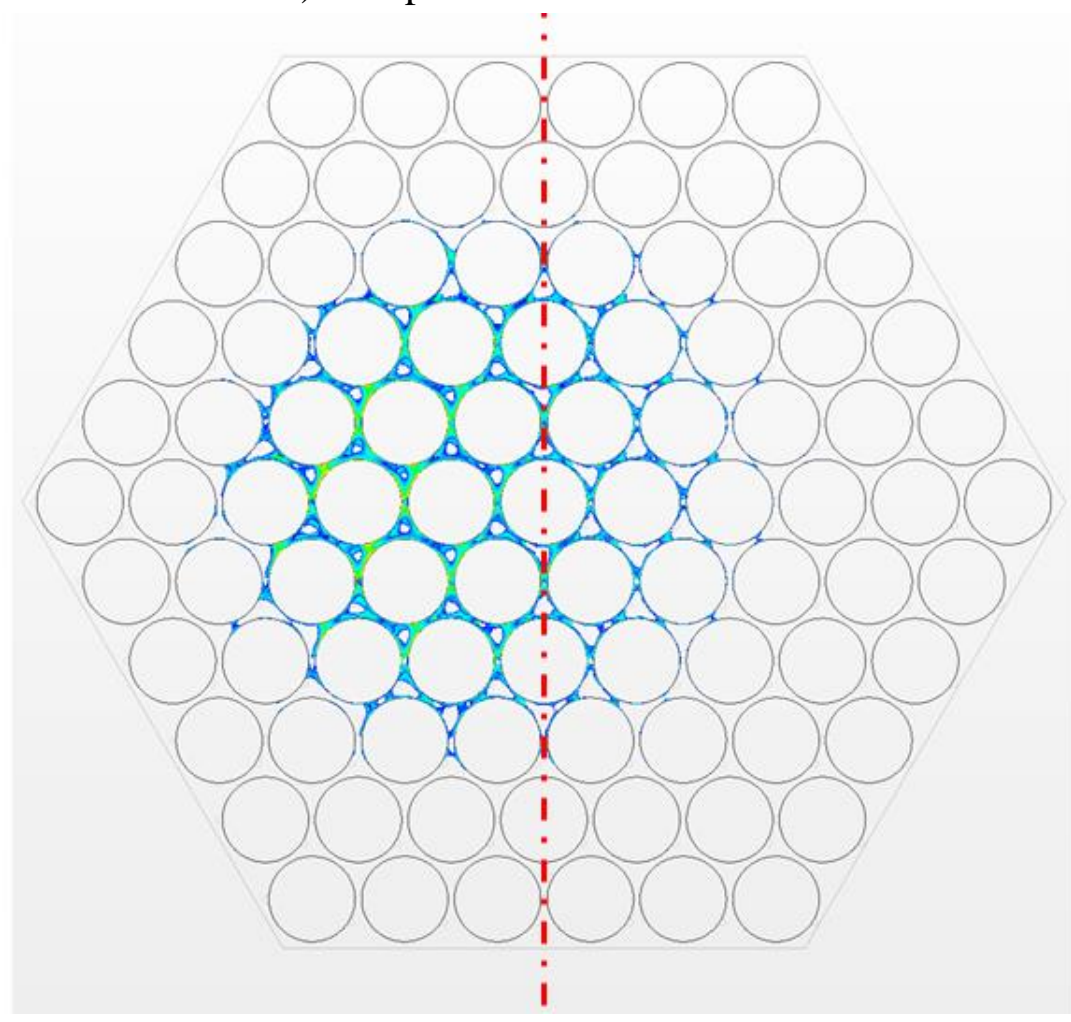

b) Overlap of the hot spots on each elevations from top view

Figure C.1.12 Asymmetric distribution feature of hot spots in wire wrap rod bundle 
Table C.1.1 Maximum coolant temperature with difference models

\begin{tabular}{|c|c|c|}
\hline Case & $\begin{array}{r}\text { Maximum Coolant } \\
\text { Temperature, }{ }^{\circ} \mathbf{C}\end{array}$ & Deviation, ${ }^{\circ} \mathbf{C}$ \\
\hline Wire wrap rod bundle & 578.61 & - \\
\hline Bare rod bundle & 585.56 & 6.95 \\
\hline Bare rod bundle & 579.84 & -1.23 \\
\hline Momentum Source & & \\
\hline
\end{tabular}

Figure C.1.13 shows the non-dimensional maximum coolant temperature in the sub-channel around the center pin. For bare rod bundle, the maximum coolant temperature in different subchannel is quite uniform. As for wire wrap rod bundle, the deviation of the maximum temperature in different sub-channel is bigger. Although the MSM model capture the magnitude of the maximum temperature well, it fails to capture the wire effect on the local temperature distribution. For instance, the maximum temperature is higher than that in other sub-channel in MSM model while it is the total opposite in wire wrap model. From some mesh sensitivity test, the results from the MSM model become suspect when the mesh is coarser than some critical point. Since the MSM cannot save as much computational resource as we expect and it also required extra coding efforts to implant the model into Nek5000, we will not use the model for further Nek5000 simulation. However, the MSM model still has the potential use to model rod bundle with different wire wrap orientation in which case the direct meshing becomes impractical.

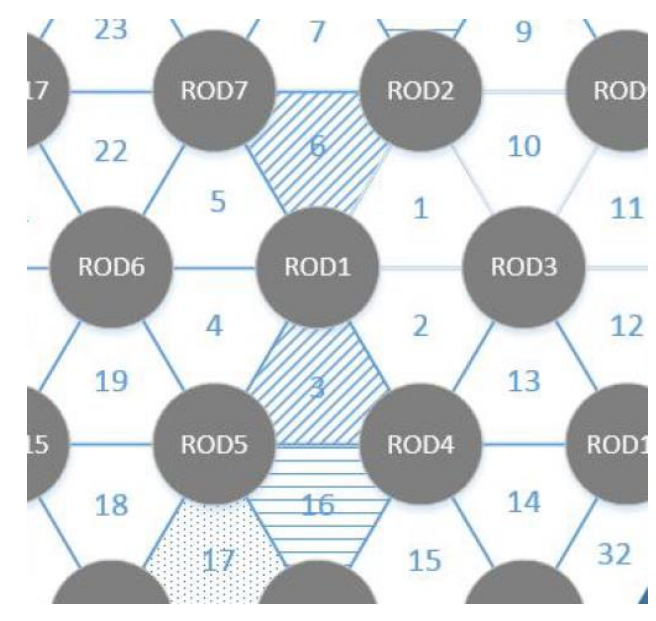

a) Sub-channel Numbering Scheme 


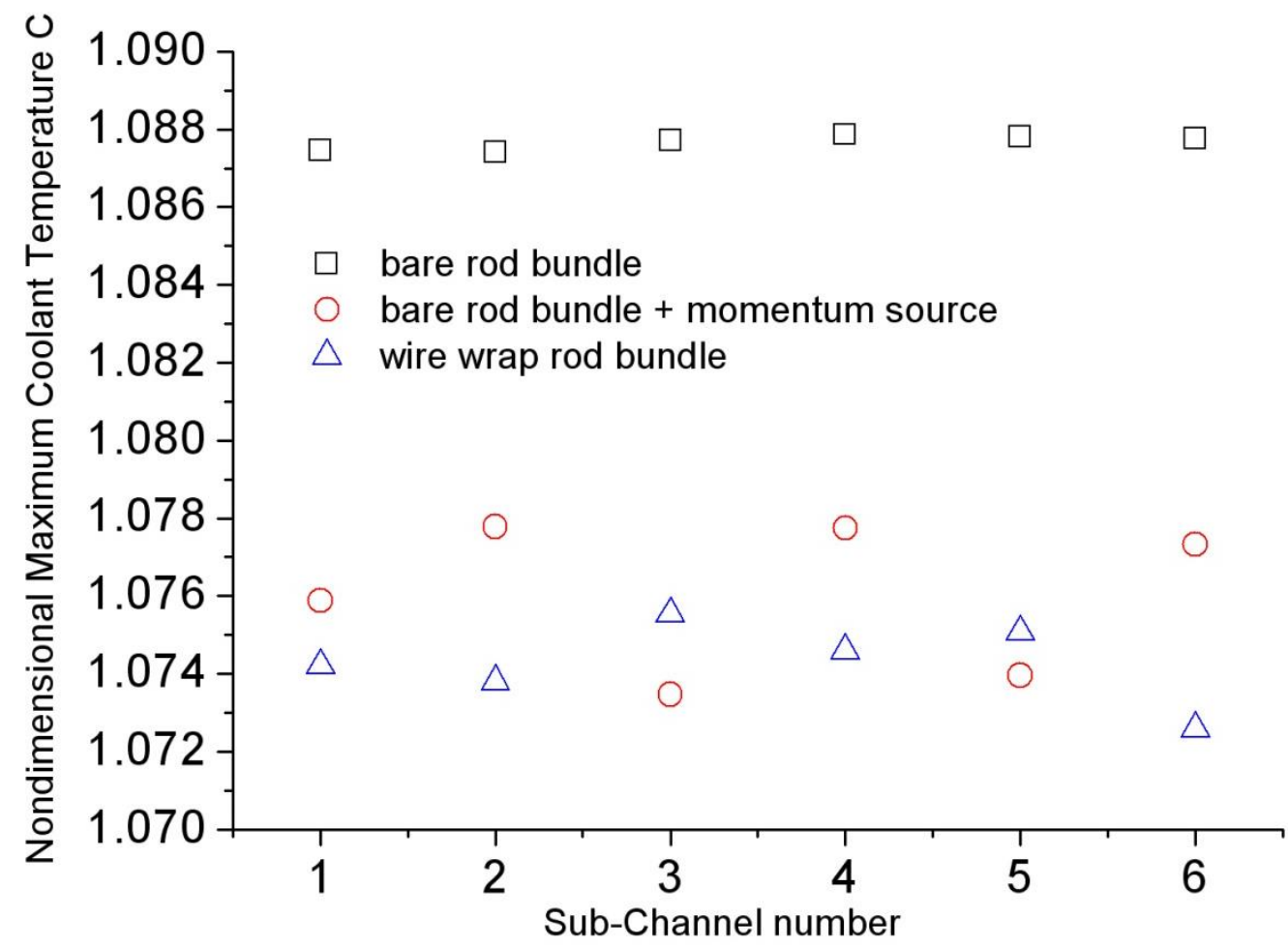

b) Sub-channel Maximum Coolant Temperature

Figure C.1.13 Maximum coolant temperature with difference models in different subchannels

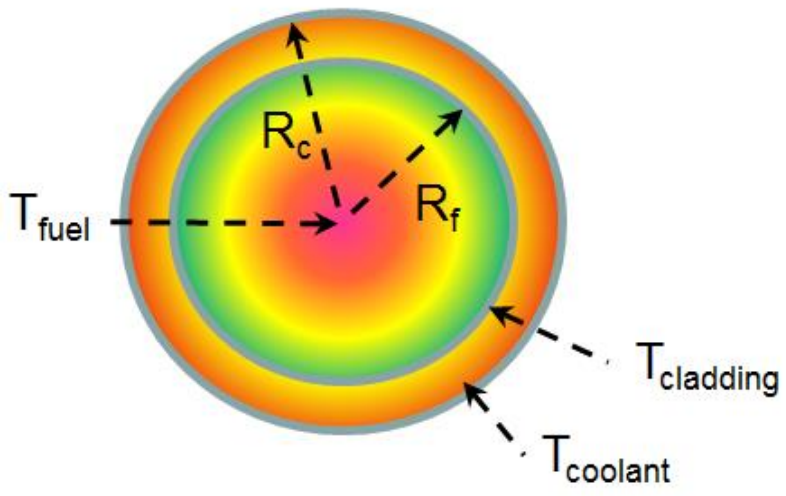

Figure C.1.14 Sketch of fuel and cladding

Conjugate Heat Transfer (CHT) is used for applications that involve simultaneous heat transfer in both solid and fluid domain. In our case, if the solid domains are not modeled simultaneously,we can get the analytic temperature solution within the solid domain through the law of heat 
conduction, also known as Fourier's law. According to Fourier's law,we can obtain the maximum cladding and fuel temerpaure derived from the flowing equations:

$$
\begin{gathered}
T_{\text {cladding_max }}=T_{\text {cladding_max }}+\frac{Q_{s} \times R_{c} \times \ln \left(R_{c} / R_{f}\right)}{k_{\text {cladding }}} \\
T_{\text {fuel_max }}=T_{\text {cladding_max }}+\frac{Q_{v} \times R_{f}{ }^{2}}{4 \times k_{\text {fuel }}}
\end{gathered}
$$

Where $Q_{s}$ is surface heat flux, $Q_{v}$ is volumetric heat flux, $R_{c}$ is cladding outer radius and $R_{f}$ is fuel radius (Figure C.1.14).Figure C.1.15 shows the temperature prediction with and without conjugate heat transfer. As shown, the maximum coolant temperature is insensitive to the conjugate heat transfer model. The model with and without conjugate hear transfer predict the same maximum temperature. Because the thermal conduction of sodium is about 100 times larger than water, the circumferential heat conduction in sodium is much stronger. It does not leave room for claddings to further homogenize the circumferential temperature since the circumferential heat transfer through sodium is already strong. Given that the thickness of the cladding is only $0.5 \mathrm{~mm}$, the maximum temperature in the cladding is also not obvious between the conjugate heat transfer calculation and the theoretical derivation based on Fourier's law. The maximum fuel temperature is over estimated by Fourier's law due to Fourier's law's one dimentisonal heat conduction asseumption. No circumferential heat conduction is taken into account by the theoretical derivation. From the temperature contour of fuel and cladding shown in Figure C.1.16, the isolines in either cladding or fuel are not absolute concentric circles, which indicates the circumferential heat conduction. The CHT further homogenize the circumferential temperature distribution. That's why the maximum fuel temperature is $4.12^{\circ} \mathrm{C}$ lower.

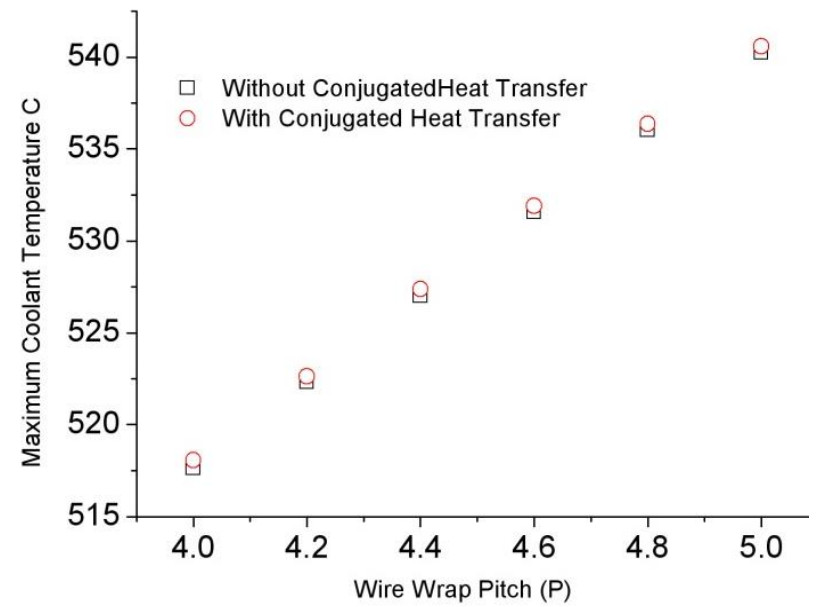

a) Maximum Coolant Temperature

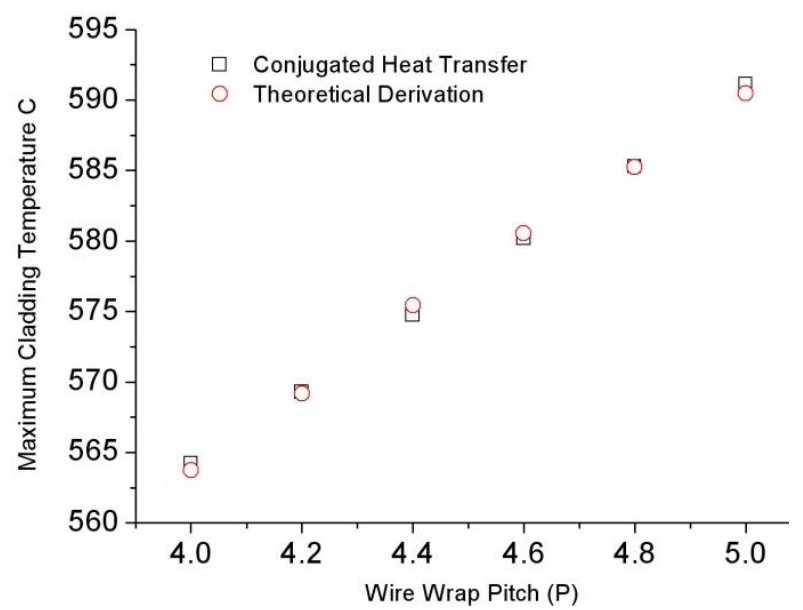

b) Maximum Cladding Temperature 


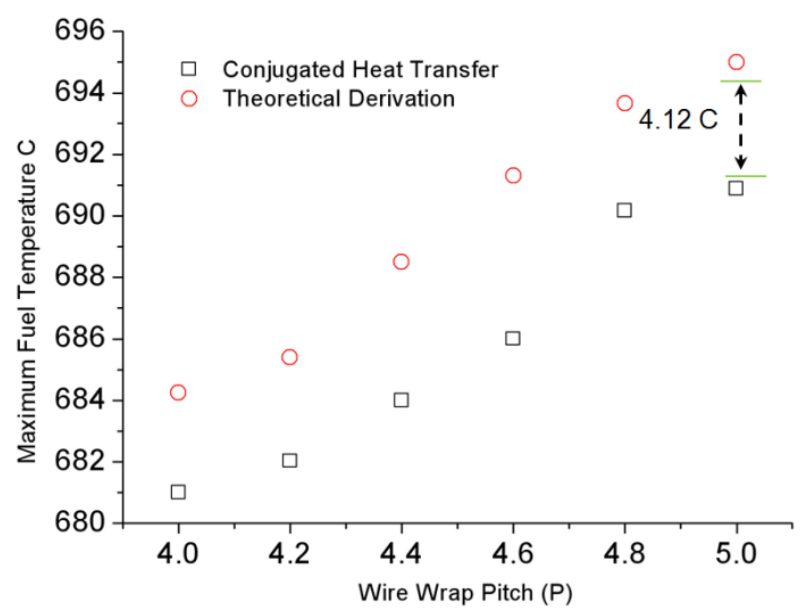

c) Maximum Fuel Temperature

\section{Figure C.1.15 Temperature prediction with and without conjugate heat transfer}

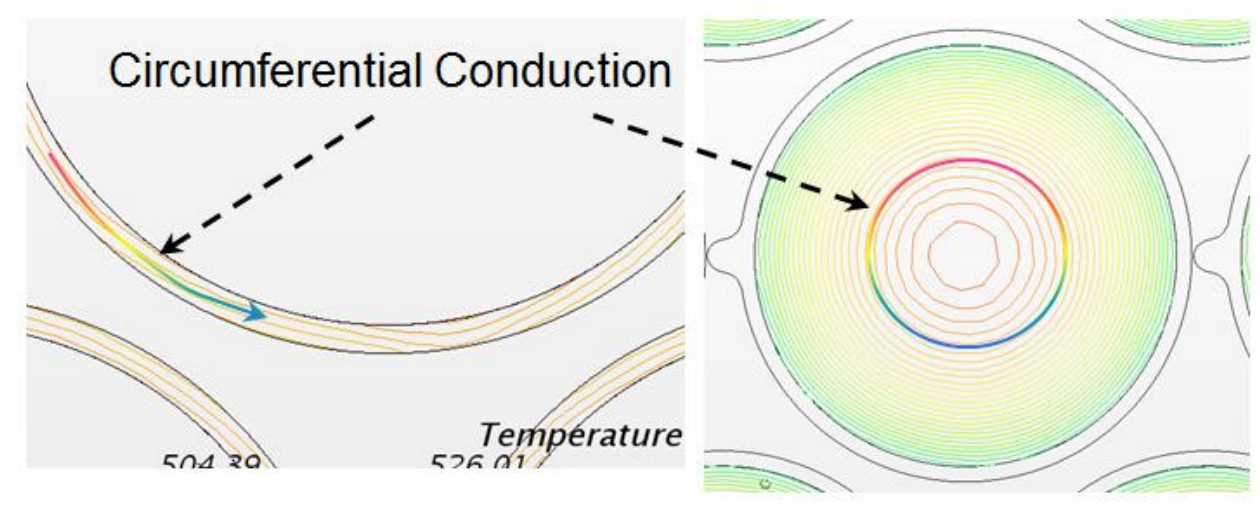

Figure C.1.16 Circumferential conduction in cladding and fuel

It's worthwhile mentioning that the CHT modeling in the fuel is impractical for Nek5000 calculation due to the difficulty in meshing for the wire wrap rod bundle. According to the primary calculation with STAR-CCM+, the bare rod bundle model can overestimate the coolant temperature by $6.95^{\circ} \mathrm{C}$ while the wire wrap rod bundle model without $\mathrm{CHT}$ can overestimate the fuel temperature by $4.12^{\circ} \mathrm{C}$. Thus, the deviation of maximum fuel temperature prediction between the bare rod bundle with CHT model and the wire wrap rod bundle without CHT model can be only $2.83{ }^{\circ} \mathrm{C}$.

Some primary simulations with different cladding thickness are performed to investigate the effect of the cladding thickness on HCF (Figure C.1.17). The thickness of the cladding does not influence the maximum cladding temperature but the deviation of the circumferential cladding temperature. Smaller cladding thickness leads to larger circumferential cladding temperature 
deviation. These primary simulations may help us to understand the effect of the cladding thickness. The information is very useful for both meshing and calculation with Nek5000.

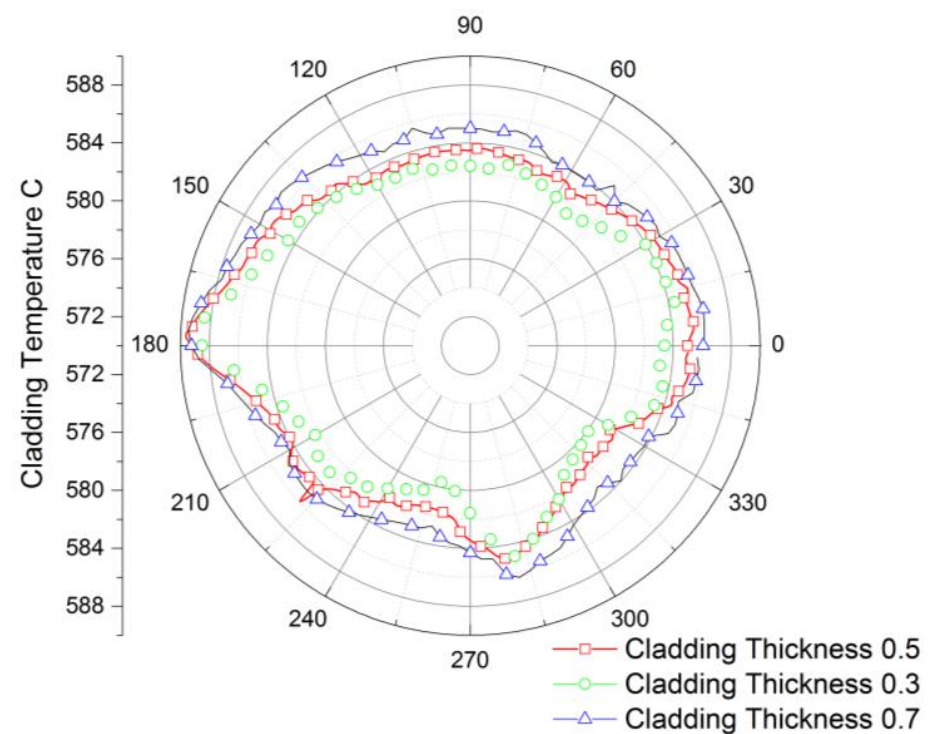

\section{Figure C.1.17 Circumferential cladding temperature distribution of the center pin with different cladding thickness}

Some conclusions can be drawn based on the STAR-CCM+ simulation introduced in this section.

- Full length domain can be simplified to 1 wire pitch domain with proper boundary conditions to reproduce the velocity and temperature distribution in the interested area.

- Conjugate Heat Transfer (CHT) does not have an obvious effect on coolant temperature prediction.

- Bare rod bundle model with CHT can be used to calculated the HCF with less than $3^{\circ} \mathrm{C}$ deviation of maximum fuel temperature prediction from that predicted by the wire wrap rod bundle model without CHT.

\section{C.2 Meshing Capability}

Advanced meshing capabilities are required for both bare rod bundle and wire wrap rod bundle with a variety of perturbations due to physical uncertainty, manufacturing tolerance, or modeling approximation. Nek5000 requires a hexahedral mesh, which needs to be taken into account before developing the meshing capability. In order to achieve that goal, two sets of meshing capabilities are developed for this purpose. The first one is a Matlab script for meshing wire wrap rod bundles. Another one is an ICEM script for meshing bare rod bundles.

For wire wrap rod bundles, the wires rotate circumferentially along the stream wise direction (Figure C.2.1). The basic meshing strategy is to generate surface mesh on each elevation and 
connect them. It has minimum layer requirements in stream wise to represent the wire wrap. Otherwise, the mesh quality can be too poor to use. Therefore, a minimum number of elements are typically required for wire wrap rod bundles. For instance, 91-pin wire wrap rod bundle of 1 pitch usually require at least 2 million elements. In general, the wire wrap rod bundle calculation is at least 10 times more computationally expensive than bare rod bundle calculation.

As for the bare rod bundle meshing capability, the following basic requirements on the script need to be satisfied for this project:

O The mesh should only consist of hexahedral quadratic elements which is adaptive to both PROTEUS and Nek5000.

- The scale of critical parameters such as rod diameter, cladding thickness and fuel diameter are adaptable

- The mesh density should be easy to control in different regions.

- The scripts should be able to provide the mesh with any naming scheme for PROTEUS.

Figure C.2.2 shows the meshes generated by ICEM script for the assemblies of different pin number. The script also provides the flexibility to adjust the mesh density locally, include the duct region and export the mesh with different format. As shown in Figure C.2.3, ICEM script can export hex 27 for Nek5000 and hex20 for PROTEUS. In addition, it creates boundary layer for Nek5000 near the wall and adds more layers in the cladding for PROTEUS.
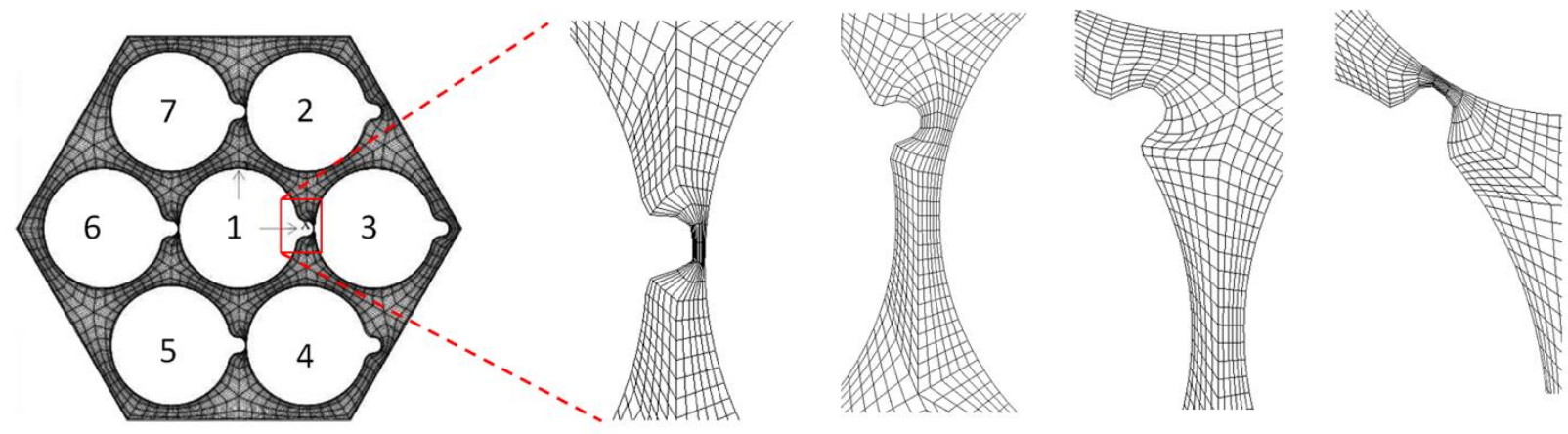

Stream wise direction

Figure C.2.1 Hexahedral mesh generation for wire wrap rod bundle 


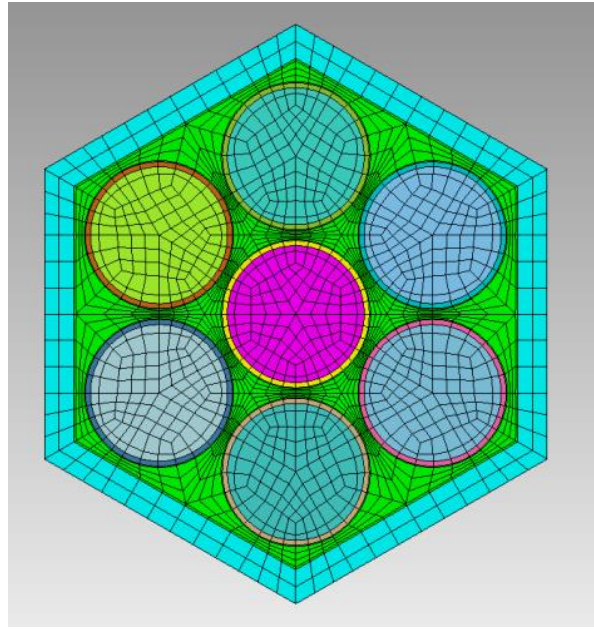

a) 7-pin

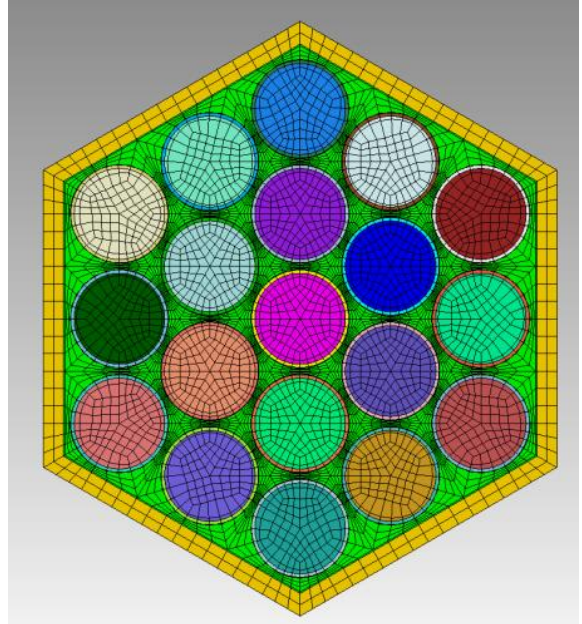

b) 19-pin
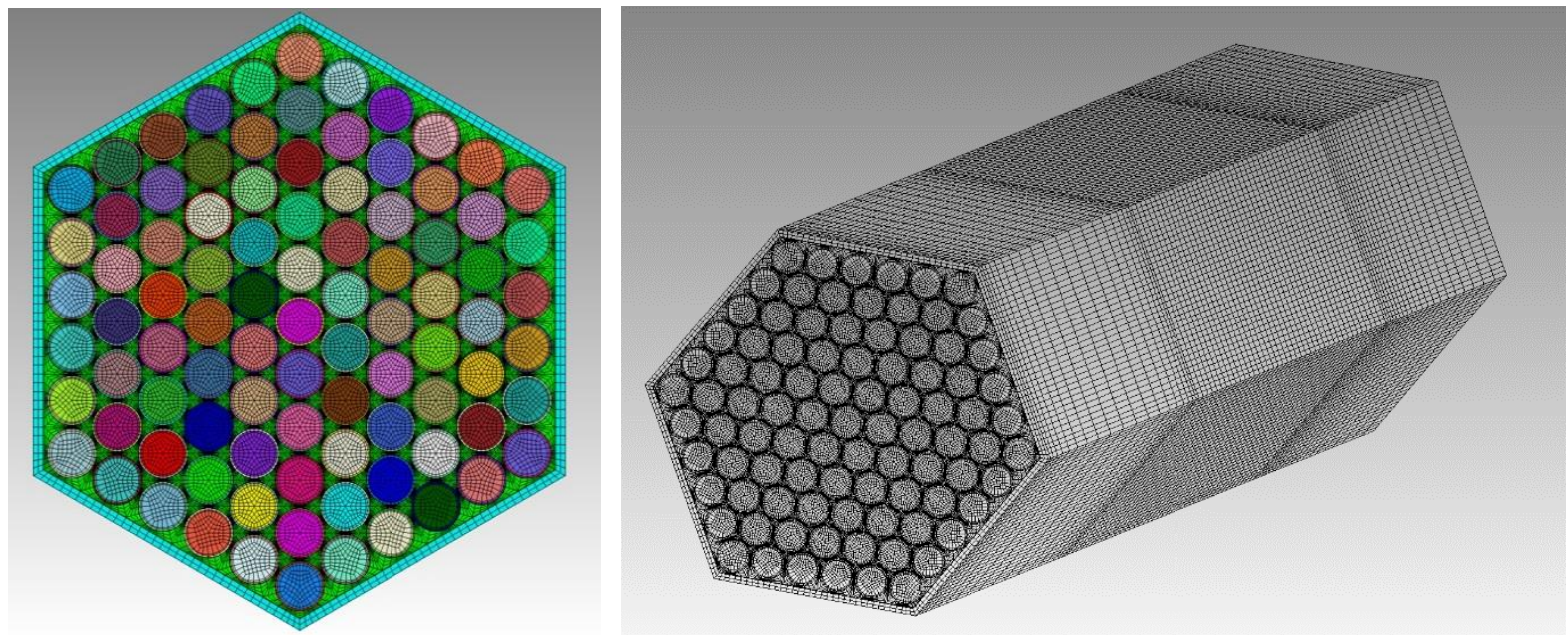

c) 91-pin

Figure C.2.2 Mesh Generation for bare rod bundle with ICEM script

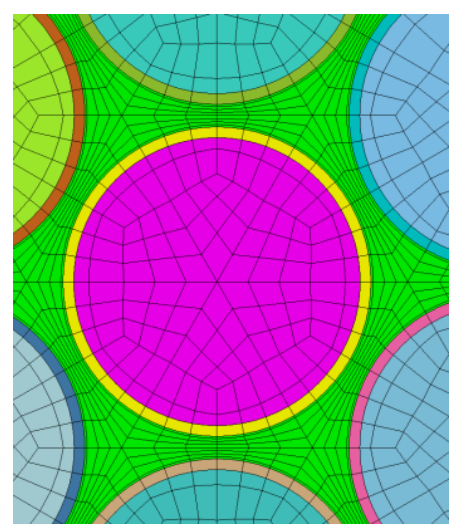

a) Nek5000 (Hex27)

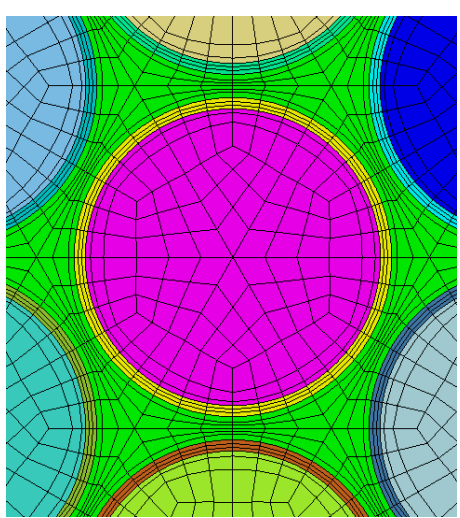

b) PROTEUS(Hex20)

Figure C.2.3 Mesh generation for different physics codes 


\section{Appendix D. Comparison of Hot Channel Factors}

The hot channel factors (HCFs) developed for CRBR, FFTF, and EBR-II have been reviewed and the recommended sets of HCFs for metallic fuel SFRs are proposed in this study. Since the HCF naming is inconsistent between three sets of HCFs, all HCF sets were lined up based on the source of uncertainties and a representative name is proposed in the recommendation. In addition, several direct hot channel factors were reclassified into statistical hot channel factors because statistical uncertainties are involved in calculations.

\begin{tabular}{|c|c|c|c|}
\hline CRBR & FFTF & EBR-II & Recommendation \\
\hline \multicolumn{4}{|c|}{ Direct factor } \\
\hline $\begin{array}{l}\text { Power level measurement } \\
\text { and control system dead } \\
\text { band } \\
\text { - "Power level } \\
\text { measurement" and } \\
\text { "control rod dead } \\
\text { band" are considered } \\
\text { simultaneously as a } \\
\text { direct factor. } \\
\text { - This HCF concept is } \\
\text { equivalent to "Control } \\
\text { rod dead band" of the } \\
\text { FFTF HCF [Friedland } \\
\text { 1980]. }\end{array}$ & $\begin{array}{l}\text { Control dead band } \\
\text { - "Control dead band" } \\
\text { is dependent on the } \\
\text { max. power } \\
\text { allowance without } \\
\text { control rod moving } \\
\text { - Control rod dead } \\
\text { band is defined in } \\
\text { "Operation Tech } \\
\text { Spec." }\end{array}$ & $\begin{array}{l}\text { Power level } \\
\text { measurement } \\
\text { - Calibration error in } \\
\text { power measurement } \\
\text { based mainly on } \\
\text { water flow rate and } \\
\text { feed water } \\
\text { temperature } \\
\text { measurement } \\
\text { uncertainties in the } \\
\text { steam cycle. }\end{array}$ & $\begin{array}{l}\text { Control rod dead band. } \\
\text { - Power level } \\
\text { measurement was } \\
\text { reclassified into } \\
\text { statistic factors } \\
\text { because major } \\
\text { uncertainties in } \\
\text { measurements of flow } \\
\text { rate, temperature, and } \\
\text { control systems are } \\
\text { statistical errors or } \\
\text { manufacturing } \\
\text { tolerance of } \\
\text { instruments. }\end{array}$ \\
\hline $\begin{array}{l}\text { Inlet Flow } \\
\text { Maldistribution } \\
\text { - Uncertainties in the } \\
\text { assembly flow } \\
\text { distribution due to } \\
\text { flow maldistribution } \\
\text { in the lower plenum, } \\
\text { manufacturing } \\
\text { tolerances in internal } \\
\text { structures and orifice, } \\
\text { etc. } \\
\text { - Direct factor of } 1.02 \\
\text { was determined based } \\
\text { on engineering } \\
\text { judgment. }\end{array}$ & $\begin{array}{l}\text { (Equivalent HCF concept } \\
\text { is Inlet Flow } \\
\text { Maldistribution, but this } \\
\text { uncertainty was treated as } \\
\text { a statistical factor) }\end{array}$ & $\begin{array}{l}\text { (Equivalent HCF concept } \\
\text { is Precision in total } \\
\text { coolant flow rate } \\
\text { through an assembly, } \\
\text { but this uncertainty was } \\
\text { treated as a statistical } \\
\text { factor) }\end{array}$ & $\begin{array}{l}\text { Reclassify this hot } \\
\text { channel factor into } \\
\text { statistic factor as like } \\
\text { FFTF and EBR-II. }\end{array}$ \\
\hline $\begin{array}{l}\text { Flow Distribution } \\
\text { Uncertainty } \\
\text { - Uncertainties of flow } \\
\text { distribution within } \\
\text { assembly. } \\
\text { - Uncertainties are } \\
\text { mainly due to the } \\
\text { simplified model and } \\
\text { applied empirical } \\
\text { factors of sub-channel } \\
\text { codes. }\end{array}$ & $\begin{array}{l}\text { (Equivalent HCF concept } \\
\text { is Intra-subassembly } \\
\text { flow distribution, but } \\
\text { this uncertainty was } \\
\text { treated as a statistical } \\
\text { factor) }\end{array}$ & $\begin{array}{l}\text { (Equivalent HCF concept } \\
\text { is Precision in total } \\
\text { coolant flow rate within } \\
\text { an assembly, but this } \\
\text { uncertainty was treated as } \\
\text { a statistical factor) }\end{array}$ & $\begin{array}{l}\text { Intra-subassembly flow } \\
\text { maldistribution } \\
\text { - Reclassify this hot } \\
\text { channel factor into } \\
\text { statistic factor }\end{array}$ \\
\hline
\end{tabular}




\begin{tabular}{|c|c|c|c|}
\hline $\begin{array}{l}\text { Cladding } \\
\text { Circumferential Temp. } \\
\text { Variation } \\
\text { - Axial coolant velocity } \\
\text { and cladding } \\
\text { temperature around a } \\
\text { fuel pin have strongly } \\
\text { azimuthal dependence } \\
\text { due to wire-wrap. } \\
\text { This HCF was } \\
\text { measured at ORNL } 7 \\
\text { and -19 pins tests and } \\
\text { firstly evaluated by } \\
\text { FATH0M-360 code } \\
\text { (NSE. 64, 1977). }\end{array}$ & $\begin{array}{l}\text { Wire-wrap Peak } \\
\text { HCF name is different, } \\
\text { but the concept is the } \\
\text { same to the CRBR's } \\
\text { Cladding Circumferential } \\
\text { Temperature Variation. }\end{array}$ & $\begin{array}{l}\text { (This uncertainty was not } \\
\text { considered) }\end{array}$ & $\begin{array}{l}\text { Cladding } \\
\text { Circumferential Temp. } \\
\text { Variation } \\
\text { - Called differently in } \\
\text { CRBR and FFTF, but } \\
\text { the concept is the } \\
\text { same. }\end{array}$ \\
\hline $\begin{array}{l}\text { Pellet-Cladding } \\
\text { Eccentricity } \\
\text { - In CRBR, it was } \\
\text { conservatively } \\
\text { calculated by } \\
\text { assuming that the } \\
\text { fresh fuel contact to } \\
\text { cladding. } \\
\text { - This uncertainty } \\
\text { impacts on cladding } \\
\text { and film temperatures. }\end{array}$ & $\begin{array}{l}\text { (This uncertainty was not } \\
\text { considered) }\end{array}$ & $\begin{array}{l}\text { (This uncertainty was not } \\
\text { considered) }\end{array}$ & $\begin{array}{l}\text { This HCF is not } \\
\text { applicable to metallic } \\
\text { fuel } \\
\text { - For oxide fuel, } \\
\text { eccentricity } \\
\text { uncertainty is } \\
\text { importance due to a } \\
\text { poor thermal } \\
\text { conductivity of gap. }\end{array}$ \\
\hline $\begin{array}{l}\text { Physics Modeling } \\
\text { - There are many sub- } \\
\text { factors under this } \\
\text { category such as flux } \\
\text { solver approximation, } \\
\text { 2D synthesis method), } \\
\text { etc. } \\
\text { - In CRBR, the lumped } \\
\text { uncertainty of the } \\
\text { power distribution } \\
\text { was estimated using } \\
\text { the ZPPR mockup of } \\
\text { CRBRP (the C/E error } \\
\text { is about +-2\% except } \\
\text { for specific locations). }\end{array}$ & $\begin{array}{l}\text { Considered as statistic } \\
\text { factor (see Nuclear Power } \\
\text { Distribution). }\end{array}$ & $\begin{array}{l}\text { (Equivalent HCF concept } \\
\text { is Precision in neutron } \\
\text { and gamma flux } \\
\text { distribution) }\end{array}$ & $\begin{array}{l}\text { Reclassify this hot } \\
\text { channel factor into } \\
\text { statistic factor } \\
\text { - For FFTF and EBR- } \\
\text { II, this HCF was } \\
\text { considered as statistic } \\
\text { factor because the } \\
\text { total power is } \\
\text { normalized to } 100 \% \\
\text { in calculation (so, not } \\
\text { directly impact on the } \\
\text { temperature) and } \\
\text { uncertainties are } \\
\text { mainly due to the } \\
\text { experimental } \\
\text { (statistical) errors. }\end{array}$ \\
\hline $\begin{array}{l}\text { Control Rod Banking } \\
\text { - Control rods are } \\
\text { grouped and there is } \\
\text { manufacturing } \\
\text { tolerance (i.e., } \\
\text { insertion depth is not } \\
\text { identical), which } \\
\text { introduces asymmetric } \\
\text { power. }\end{array}$ & $\begin{array}{l}\text { (This uncertainty was not } \\
\text { considered) }\end{array}$ & $\begin{array}{l}\text { (This uncertainty was not } \\
\text { considered) }\end{array}$ & $\begin{array}{l}\text { Control Rod Banking } \\
\text { - This is a direct factor } \\
\text { because peak } \\
\text { temperature increases } \\
\text { as much as bank } \\
\text { asymmetric } \\
\text { uncertainty. } \\
\text { - This is dependent on } \\
\text { design requirement. } \\
\text { For CRBR, tolerance } \\
\text { is }+-1.5 \text { inches, }\end{array}$ \\
\hline
\end{tabular}




\begin{tabular}{|c|c|c|c|}
\hline & & & $\begin{array}{l}\text { which affects about } \\
2 \% \text {. }\end{array}$ \\
\hline \multicolumn{4}{|l|}{ Statistical factors } \\
\hline $\begin{array}{l}\text { Reactor delta-T and inlet } \\
\text { temperature variation } \\
\text { - Uncertainties of } \\
\text { reactor delta-T due to } \\
\text { deterioration of } \\
\text { primary components. } \\
\text { - This HCF was } \\
\text { formulated in terms of } \\
\text { inlet temperature, } \\
\text { reactor delta-T and } \\
\text { outlet temperature. }\end{array}$ & $\begin{array}{l}\text { Primary Sodium } \\
\text { Temperature } \\
\text { Measurement }\end{array}$ & $\begin{array}{l}\text { (This uncertainty was not } \\
\text { considered) }\end{array}$ & $\begin{array}{l}\text { Reactor delta-T and inlet } \\
\text { temperature variation }\end{array}$ \\
\hline $\begin{array}{l}\text { Inlet Flow } \\
\text { Maldistribution } \\
\text { - Uncertainties of } \\
\text { pressure } \\
\text { measurement, } \\
\text { manufacturing } \\
\text { tolerance, orifice flow } \\
\text { rate, assembly flow } \\
\text { rate, etc. }\end{array}$ & $\begin{array}{l}\text { Inlet Flow } \\
\text { Maldistribution }\end{array}$ & $\begin{array}{l}\text { Precision in total coolant } \\
\text { flow rate through } \\
\text { assemblies }\end{array}$ & $\begin{array}{l}\text { Inlet Flow } \\
\text { Maldistribution } \\
\text { - Consistently count } \\
\text { this uncertainty in } \\
\text { three reactors with } \\
\text { comparable HCF } \\
\text { values. }\end{array}$ \\
\hline $\begin{array}{l}\text { Loop Temperature } \\
\text { Imbalance } \\
\text { - Loop temperature } \\
\text { imbalance affects inlet } \\
\text { temperature } \\
\text { distribution. } \\
\text { - CRBR allows cold leg } \\
\text { loop-to-loop } \\
\text { temperature } \\
\text { imbalance, which } \\
\text { results in uncertainty } \\
\text { in inlet temperature. }\end{array}$ & $\begin{array}{l}\text { (This uncertainty was not } \\
\text { considered) }\end{array}$ & $\begin{array}{l}\text { (This uncertainty was not } \\
\text { considered) }\end{array}$ & $\begin{array}{l}\text { Loop Temperature } \\
\text { Imbalance }\end{array}$ \\
\hline $\begin{array}{l}\text { Wire-wrap Orientation } \\
\text { - Analyzed by } \\
\text { subchannel code, } \\
\text { COTEC, which is } 1 \% \\
\text { uncertainty. }\end{array}$ & $\begin{array}{l}\text { (This uncertainty was not } \\
\text { considered) }\end{array}$ & Wire-wrap Orientation & Wire-wrap Orientation \\
\hline $\begin{array}{l}\text { Subchannel Flow Area } \\
\text { - Uncertainties of rod } \\
\text { dimension tolerance, } \\
\text { bow, etc. }\end{array}$ & $\begin{array}{l}\text { Rod Diameter, Pitch } \\
\text { and Bow }\end{array}$ & $\begin{array}{l}\text { (This uncertainty was } \\
\text { partially counted in fuel } \\
\text { thermal conductivity } \\
\text { HCF) }\end{array}$ & Subchannel Flow Area \\
\hline $\begin{array}{l}\text { Film Heat Transfer } \\
\text { Coefficient } \\
\text { - Uncertainties of } \\
\text { correlation, etc. }\end{array}$ & Film Coefficient & $\begin{array}{l}\text { Film Heat Transfer } \\
\text { Coefficient }\end{array}$ & $\begin{array}{l}\text { Film Heat Transfer } \\
\text { Coefficient }\end{array}$ \\
\hline $\begin{array}{l}\text { Pellet-Cladding } \\
\text { Eccentricity } \\
\text { - In CRBR, it was } \\
\text { conservatively } \\
\text { calculated by }\end{array}$ & $\begin{array}{l}\text { (This uncertainty was not } \\
\text { considered) }\end{array}$ & $\begin{array}{l}\text { (This uncertainty was not } \\
\text { considered) }\end{array}$ & $\begin{array}{l}\text { For oxide fuel, it may } \\
\text { be importance due to } \\
\text { a poor thermal } \\
\text { conductivity of gap, } \\
\text { but this HCF is not }\end{array}$ \\
\hline
\end{tabular}




\begin{tabular}{|c|c|c|c|}
\hline $\begin{array}{l}\text { assuming that the } \\
\text { fresh fuel contact to } \\
\text { cladding. } \\
\text { - This uncertainty } \\
\text { impacts on cladding } \\
\text { and film temperatures. }\end{array}$ & & & $\begin{array}{l}\text { applicable to metallic } \\
\text { fuel. }\end{array}$ \\
\hline $\begin{array}{l}\text { Cladding Thickness and } \\
\text { Conductivity }\end{array}$ & & $\begin{array}{l}\text { Cladding thermal } \\
\text { conductivity and } \\
\text { thickness manufacturing } \\
\text { deviation }\end{array}$ & Cladding properties \\
\hline Coolant Properties & $\begin{array}{l}\text { (This uncertainty was not } \\
\text { considered) }\end{array}$ & Coolant Properties & Coolant Properties \\
\hline $\begin{array}{l}\text { Flow Distribution } \\
\text { Calculation } \\
\text { - The flow and } \\
\text { temperature } \\
\text { distributions were } \\
\text { calculated using } \\
\text { subchannel code of } \\
\text { COBRA, COTEC, } \\
\text { and THI-3D. }\end{array}$ & $\begin{array}{l}\text { Intra-assembly Flow } \\
\text { Maldistribution } \\
\text { Calculation }\end{array}$ & $\begin{array}{l}\text { Precision in Coolant } \\
\text { Flow Profile within an } \\
\text { Assembly }\end{array}$ & $\begin{array}{l}\text { Intra-assembly Flow } \\
\text { Maldistribution } \\
\text { Calculation } \\
\text { - Magnitude of } \\
\text { uncertainty is } \\
\text { dependent on the code } \\
\text { performance. }\end{array}$ \\
\hline Nuclear Data & $\begin{array}{l}\text { Nuclear Power } \\
\text { Distribution }\end{array}$ & $\begin{array}{l}\text { Neuron and gamma flux } \\
\text { distribution }\end{array}$ & $\begin{array}{l}\text { Physics modeling } \\
\text { - Modeling and } \\
\text { simulation } \\
\text { uncertainties. } \\
\end{array}$ \\
\hline \multirow{2}{*}{$\begin{array}{l}\text { Fissile fuel } \\
\text { maldistribution } \\
\text { - Due to manufacturing } \\
\text { tolerance. }\end{array}$} & Fissile maldistribution & Fissile content & Fissile maldistribution \\
\hline & & $\begin{array}{l}\text { Fuel Thermal } \\
\text { Conductivity } \\
\text { - Uncertainties due to } \\
\text { pellet diameter, fresh } \\
\text { and irradiated fuel } \\
\text { conductivity, porosity } \\
\text { of swollen fuel, } \\
\text { redistribution, etc. }\end{array}$ & $\begin{array}{l}\text { Fuel Thermal } \\
\text { Conductivity }\end{array}$ \\
\hline
\end{tabular}




\section{Argonne}

Nuclear Science and Engineering Division

Argonne National Laboratory

9700 South Cass Avenue, Bldg. 208

Argonne, IL 60439

www.anl.gov

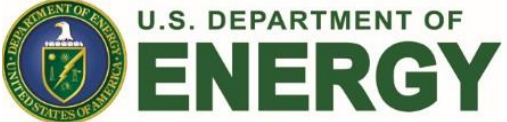

